\title{
Physics of Neutron Star Crusts
}

\author{
Nicolas Chamel \\ Institut d'Astronomie et d'Astrophysique \\ Université Libre de Bruxelles \\ CP226 \\ Boulevard du Triomphe B-1050 Brussels, Belgium \\ email: nchamel@ulb.ac.be \\ http://www-astro.ulb.ac.be/ \\ Pawel Haensel \\ Nicolaus Copernicus Astronomical Center \\ Polish Academy of Sciences \\ Bartycka 18, \\ 00-716 Warszawa, Poland \\ email: haensel@camk.edu.pl \\ http://www. camk. edu.pl

\section{Living Reviews in Relativity \\ ISSN 1433-8351}

Accepted on 5 September 2008

Published on 12 December 2008

\begin{abstract}
The physics of neutron star crusts is vast, involving many different research fields, from nuclear and condensed matter physics to general relativity. This review summarizes the progress, which has been achieved over the last few years, in modeling neutron star crusts, both at the microscopic and macroscopic levels. The confrontation of these theoretical models with observations is also briefly discussed.
\end{abstract}

This review is licensed under a Creative Commons Attribution-Non-Commercial-NoDerivs 3.0 Germany License. http://creativecommons.org/licenses/by-nc-nd/3.0/de/ 


\section{Imprint / Terms of Use}

Living Reviews in Relativity is a peer reviewed open access journal published by the Max Planck Institute for Gravitational Physics, Am Mühlenberg 1, 14476 Potsdam, Germany. ISSN 1433-8351.

This review is licensed under a Creative Commons Attribution-Non-Commercial-NoDerivs 3.0

Germany License: http://creativecommons.org/licenses/by-nc-nd/3.0/de/

Because a Living Reviews article can evolve over time, we recommend to cite the article as follows:

Nicolas Chamel and Pawel Haensel,

"Physics of Neutron Star Crusts",

Living Rev. Relativity, 11, (2008), 10. [Online Article]: cited [<date $>$, http://www.livingreviews.org/lrr-2008-10

The date given as $<$ date $>$ then uniquely identifies the version of the article you are referring to.

\section{Article Revisions}

Living Reviews supports two different ways to keep its articles up-to-date:

Fast-track revision A fast-track revision provides the author with the opportunity to add short notices of current research results, trends and developments, or important publications to the article. A fast-track revision is refereed by the responsible subject editor. If an article has undergone a fast-track revision, a summary of changes will be listed here.

Major update A major update will include substantial changes and additions and is subject to full external refereeing. It is published with a new publication number.

For detailed documentation of an article's evolution, please refer always to the history document of the article's online version at http://www.livingreviews.org/lrr-2008-10. 


\section{Contents}

1 Introduction $r$

2 Plasma Parameters $\quad 11$

2.1 No magnetic field . . . . . . . . . . . . . . . . . . . . . . . . . . 11

2.2 Effects of magnetic fields . . . . . . . . . . . . . . . . . . . . . . 14

3 The Ground State Structure of Neutron Star Crusts 16

3.1 Structure of the outer crust . . . . . . . . . . . . . . . . . . 16

3.2 Structure of the inner crust . . . . . . . . . . . . . . . . . . . . . . . . 19

3.2.1 Liquid drop models . . . . . . . . . . . . . . . . . . . . . . . . . . . . . . . . . . . . . . . . . .

3.2.2 Semi-classical models . . . . . . . . . . . . . . . . . . . . 23

3.2.3 Quantum calculations . . . . . . . . . . . . . . . . 26

3.2 .4 Going further: nuclear band theory . . . . . . . . . . . . . . . . . 31

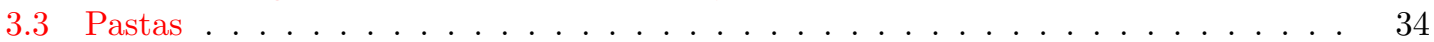

3.4 Impurities and defects . . . . . . . . . . . . . . . . . 37

4 Accreting Neutron Star Crusts $\quad 38$

4.1 Accreting neutron stars in low-mass X-ray binaries . . . . . . . . . . . . . . . . . . . . 38

4.2 Nuclear processes and formation of accreted crusts . . . . . . . . . . . . . . . . . . . . . . . . . . . . . . . . . . . . .

4.3 Deep crustal heating . . . . . . . . . . . . . . . . . . . . . . . . . 42

4.4 Thermal structure of accreted crusts and X-ray bursts . . . . . . . . . . . . 46

5 Equation of State $\quad 48$

5.1 Ground state crust . . . . . . . . . . . . . . . . . . . . 48

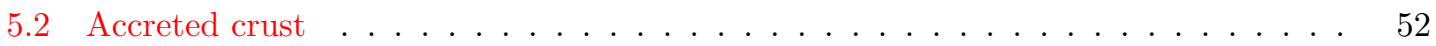

5.3 Effect of magnetic fields on the EoS . . . . . . . . . . . . . . . 53

5.4 Supernova core at subnuclear density . . . . . . . . . . . . . . . . . 53

6 Crust in Global Neutron Star Structure $\quad \mathbf{5 6}$

6.1 Spherical nonrotating neutron stars . . . . . . . . . . . . . . . . 57

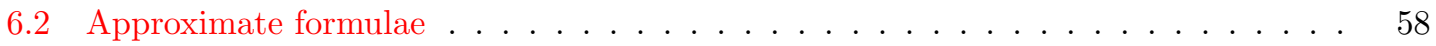

6.3 Crust in rotating neutron stars . . . . . . . . . . . . . . . . . . 60

6.4 Effects of magnetic fields on the crust structure . . . . . . . . . . . . . . 63

7 Elastic Properties $\quad \mathbf{6 5}$

7.1 Isotropic solid (polycrystal) . . . . . . . . . . . . . . . . . 66

7.2 Nuclear pasta . . . . . . . . . . . . . . . . . . . 68

8 Superfluidity and Superconductivity $\quad \mathbf{7 0}$

8.1 Superconductivity in neutron star crusts . . . . . . . . . . . . . . . . . . . . 70

8.2 Static properties of neutron superfluidity . . . . . . . . . . . . . . . 71

8.2.1 Neutron pairing gap in uniform neutron matter at zero temperature . . . . 72

8.2.2 Critical temperature for neutron superfluidity . . . . . . . . . . . . . 76

8.2.3 Pairing gap in neutron star crusts . . . . . . . . . . . . . . . . . . 77

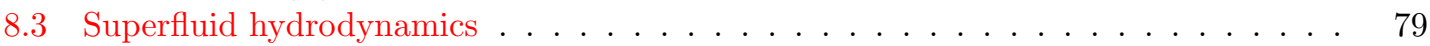

8.3.1 Superflow and critical velocity . . . . . . . . . . . . . . . . 79

8.3.2 Rotating superfluid and vortices . . . . . . . . . . . . . . . 83

8.3.3 Type II superconductors and magnetic flux tubes . . . . . . . . . . . . 85

8.3.4 Superfluid vortices and magnetic flux tubes in neutron stars . . . . . . . . 86 
8.3.5 Dynamics of superfluid vortices . . . . . . . . . . . . . . 86

8.3.6 Superfluid hydrodynamics and entrainment . . . . . . . . . . . . . . 88

8.3.7 Entrainment effects in neutron stars . . . . . . . . . . . . . . . 90

9 Conductivity and Viscosity $\quad 92$

9.1 Introduction . . . . . . . . . . . . . . . . . . . . . . . . 92

9.2 Boltzmann equation for electrons and its solutions . . . . . . . . . . . . . . . 92

9.3 Thermal and electrical conductivities . . . . . . . . . . . . . . . . . . . 95

9.4 Viscosity . . . . . . . . . . . . . . . . . . . . . . . . 97

9.5 Transport in the presence of strong magnetic fields . . . . . . . . . . . . . . . 100

9.5.1 Nonquantizing magnetic fields . . . . . . . . . . . . . . . . . . . . . . . . 101

9.5.2 Weakly-quantizing magnetic fields . . . . . . . . . . . . . . . . . . 101

9.5.3 Strongly-quantizing magnetic fields . . . . . . . . . . . . . . . . . . . 102

9.5.4 Possible dominance of ion conduction . . . . . . . . . . . . . . . 102

10 Macroscopic Model of Neutron Star Crusts 103

10.1 Variational formulation of multi-fluid hydrodynamics . . . . . . . . . . . . . 103

10.2 Two-fluid model of neutron star crust . . . . . . . . . . . . . . . . . . . . . . 104

10.3 Entrainment and effective masses . . . . . . . . . . . . . . . . . . . . . . . . . . . . . . . . . . . . . . . . . . . .

10.4 Neutron superfluidity . . . . . . . . . . . . . . . . . . . . 107

11 Neutrino Emission $\quad 110$

11.1 Neutrino emission processes - an overview . . . . . . . . . . . . . . . . . 110

11.2 Electron-positron pair annihilation . . . . . . . . . . . . . . . . . 111

11.3 Plasmon decay . . . . . . . . . . . . . . . . . . . . . . . . 112

11.4 Photoneutrino emission . . . . . . . . . . . . . . . . . . . . 112

11.5 Neutrino Bremsstrahlung from electron-nucleus collisions . . . . . . . . . . . . 112

11.6 Cooper pairing of neutrons . . . . . . . . . . . . . . . . . . . . 113

11.7 Synchrotron radiation from electrons . . . . . . . . . . . . . . . . . . . 114

11.8 Other neutrino emission mechanisms . . . . . . . . . . . . . . . . . . . 114

11.8.1 Direct Urca process in the pasta phase of the crust . . . . . . . . . . . . 114

11.9 Neutrinos from the crust - summary in the $T-\rho$ plane . . . . . . . . . . . . 115

12 Observational Constraints on Neutron Star Crusts 119

12.1 Supernovae and the physics of hot dense inhomogeneous matter . . . . . . . . . 119

12.2 Cooling of isolated neutron stars . . . . . . . . . . . . . . . . . . . . . . . . . . . . . . . . . . . . . .

12.2.1 Thermal relaxation of the crust . . . . . . . . . . . . . . . . . 121

12.2.2 Observational constraints from thermal X-ray emission . . . . . . . . . . . . 124

12.3 r-process in the decompression of cold neutron star crusts . . . . . . . . . . . . . . 124

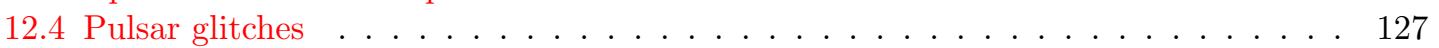

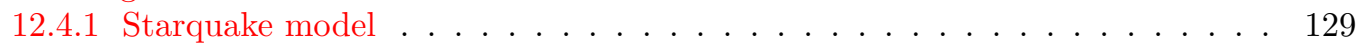

12.4 .2 Two-component models . . . . . . . . . . . . . . . . . . 130

12.4.3 Recent theoretical developments . . . . . . . . . . . . . . . . . . . . . . . . . . . . . . . . . . .

12.4.4 Pulsar glitch constraints on neutron star structure . . . . . . . . . . . . 132

12.5 Gravitational wave asteroseismology . . . . . . . . . . . . . . . . . . . . . . . . . . . . . . . . . . . . . . .

12.5.1 Mountains on neutron stars . . . . . . . . . . . . . . . . . 134

12.5.2 Oscillations and precession . . . . . . . . . . . . . . . . . 136

12.5.3 Crust-core boundary and r-mode instability . . . . . . . . . . . . . . . . . 137

12.6 Giant flares from Soft Gamma Repeaters . . . . . . . . . . . . . . . . . . . 137

12.7 Low mass X-ray binaries . . . . . . . . . . . . . . . . . . . . . . . 139

12.7 .1 Burst oscillations . . . . . . . . . . . . . . . . . . . . 140 
12.7.2 Soft X-ray transients in quiescence . . . . . . . . . . . . . . . . . . 142

12.7.3 Initial cooling in quasi-persistent SXTs . . . . . . . . . . . . . . . 144

13 Conclusion $r$

14 Acknowledgments $\quad 147$

$\begin{array}{lr}\text { A List of Notations } & 148\end{array}$

$\begin{array}{lr}\text { B List of Abbreviations } & 150\end{array}$

$\begin{array}{lr}\text { References } & 151\end{array}$

\section{List of Tables}

1 Critical temperature (in $\mathrm{K}$ ) below which a condensed phase exists at $P=0$, for several magnetic field strengths $B_{12}=B / 10^{12} \mathrm{G}$ and matter compositions. . . . .

2 Sequence of nuclei in the ground state of the outer crust of a neutron star calculated by Rüster et al. . . . . . . . . . . . . . . . . . . . . . . .

3 Sequence of nuclear clusters in the ground state of the inner crust calculated by Negele \& Vautherin. . . . . . . . . . . . . . . . . . . . . . . . .

4 Sequence of nuclear clusters in the ground state of the inner crust calculated by Baldo et al. . . . . . . . . . . . . . . . . . .

5 Parameters for the analytic formula of a few representative ${ }^{1} S_{0}$ pairing gaps in pure neutron matter. . . . . . . . . . . . . . . . . . . . . . . 75

6 Main neutrino emission processes in neutron star crusts. . . . . . . . . . . . . . . 110 



\section{Introduction}

Constructing models of neutron stars requires knowledge of the physics of matter with a density significantly exceeding the density of atomic nuclei. The simplest picture of the atomic nucleus is a drop of highly incompressible nuclear matter. Analysis of nuclear masses tells us that nuclear matter at saturation (i.e. at the minimum of the energy per nucleon) has the density $\rho_{0}=2.8 \times$ $10^{14} \mathrm{~g} \mathrm{~cm}^{-3}$, often called normal nuclear density. It corresponds to $n_{0}=0.16$ nucleons per fermi cubed. The density in the cores of massive neutron stars is expected to be as large as $\sim 5-10 \rho_{0}$ and in spite of decades of observations of neutron stars and intense theoretical studies, the structure of the matter in neutron star cores and in particular its equation of state remain the well-kept secret of neutron stars (for a recent review, see the book by Haensel, Potekhin and Yakovlev [184]). The physics of matter with $\rho \sim 5-10 \rho_{0}$ is a huge challenge to theorists, with observations of neutron stars being crucial for selecting a correct dense-matter model. Up to now, progress has been slow and based overwhelmingly on scant observation [184].

The outer layer of neutron stars with density $\rho<\rho_{0}-$ the neutron star crust - which is the subject of the present review, represents very different theoretical challenges and observational opportunities. The elementary constituents of the matter are neutrons, protons, and electrons like in the atomic matter around us. The density is "subnuclear", so that the methods developed and successfully applied in the last decades to terrestrial nuclear physics can be applied to neutron star crusts. Of course, the physical conditions are extreme and far from terrestrial ones. The compression of matter by gravity crushes atoms and forces, through electron captures, the neutronization of the matter. This effect of huge pressure was already predicted in the 1930s (Sterne [390], Hund [202, 203]). At densities $\rho \gtrsim 4 \times 10^{11} \mathrm{~g} \mathrm{~cm}^{-3}$ a fraction of the neutrons is unbound and forms a gas around the nuclei. For a density approaching $10^{14} \mathrm{~g} \mathrm{~cm}^{-3}$, some $90 \%$ of nucleons are neutrons while nuclei are represented by proton clusters with a small neutron fraction. How far we are taken from terrestrial nuclei with a moderate neutron excess! Finally, somewhat above $10^{14} \mathrm{~g} \mathrm{~cm}^{-3}$ nuclei can no longer exist - they coalesce into a uniform plasma of nearly-pure neutron matter, with a few percent admixture of protons and electrons: we reach the bottom of the neutron star crust.

The crust contains only a small percentage of a neutron star's mass, but it is crucial for many astrophysical phenomena involving neutron stars. It contains matter at subnuclear density, and therefore there is no excuse for the theoretical physicists, at least in principle: the interactions are known, and many-body theory techniques are available. Neutron star crusts are wonderful cosmic laboratories in which the full power of theoretical physics can be demonstrated and hopefully confronted with neutron star observations.

To construct neutron star crust models we have to employ atomic and plasma physics, as well as the theory of condensed matter, the physics of matter in strong magnetic fields, the theory of nuclear structure, nuclear reactions, the nuclear many-body problem, superfluidity, physical kinetics, hydrodynamics, the physics of liquid crystals, and the theory of elasticity. Theories have to be applied under extreme physical conditions, very far from the domains where they were originally developed and tested. Therefore, caution is a must!

Most of this review is devoted to theoretical descriptions of various aspects of neutron star crusts. The plural "crusts" in the title is well justified; depending on the scenario of their formations, the crusts may be very different in their composition and structure as sketched in Figure 1. In Section 2 we briefly describe the basic plasma parameters relevant to crust physics and delineate various plasma regimes in the temperature-density plane. We also address the important question of the magnetic field.

The ground state of the crust (one of the possible "crusts") is reviewed in Section 3. We also discuss there the uncertainties concerning the densest bottom layers of the crust and we mention possible deviations from the ground state. As we describe in Section 4, a crust formed via accretion 

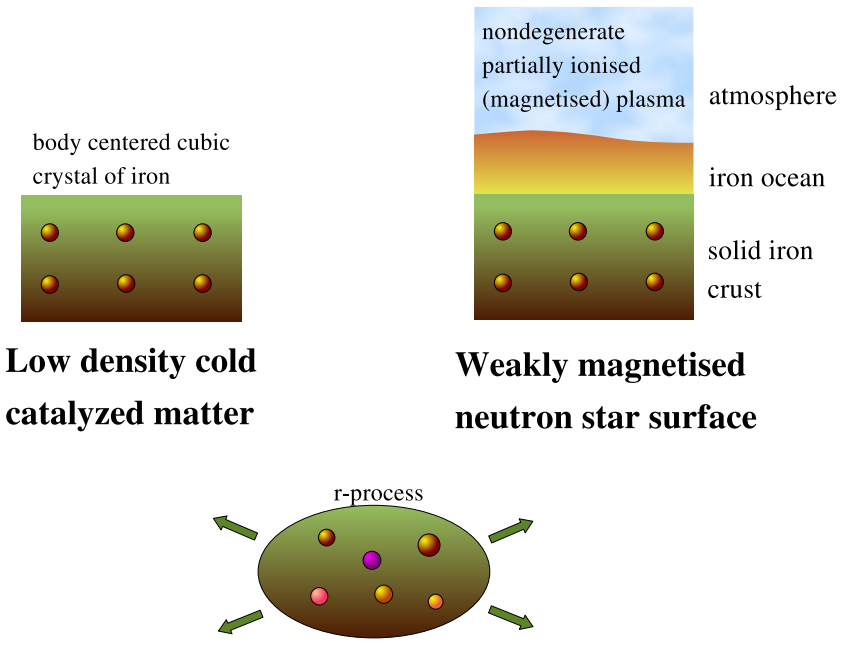

Ejected cold decompressed
neutron star crust matter

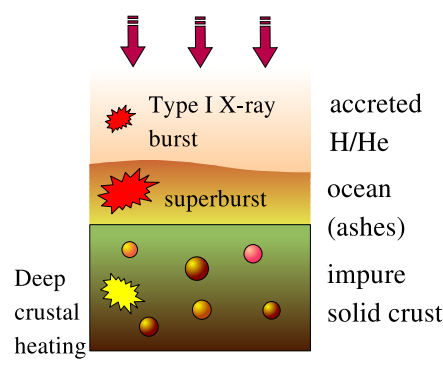

Accreting neutron star surface

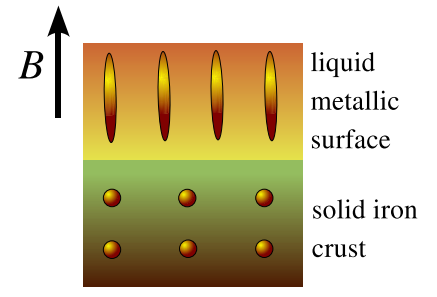

Strongly magnetised neutron star surface

Figure 1: Schematic pictures of various neutron star surfaces. 
is expected to be very different from that formed during the aftermath of a supernova explosion. We show how it can be a site for nuclear reactions. We study its thermal structure during accretion, and briefly review the phenomenon of X-ray bursts. We quantitatively analyze the phenomenon of deep crustal heating.

To construct a neutron star model one needs the equation of state (EoS) of the crust, reviewed in Section 5. We consider separately the ground-state crust, and the accreted crust. For the sake of comparison, we also describe another EoS of matter at subnuclear density - that relevant for the collapsing type II supernova core.

Section 6 is devoted to the stellar-structure aspects of neutron star crusts. We start with the simplest case of a spherically-symmetric static neutron star and derive approximate formulae for crust mass and moment of inertia. Then we study the deformation of the crust in a rotating star. Finally, we consider the effects of magnetic fields on the crust structure. Apart from isotropic stress resulting from pressure, a solid crust can support an elastic strain. Elastic properties are reviewed in Section 7. A separate subsection is devoted to the elastic parameters of the so-called "pasta" layers, which behave like liquid crystals. The inner crust is permeated by a neutron superfluid. Various aspects of crustal superfluidity are reviewed in Section 8. After a brief introduction to superconductivity and its relevance for neutron star crusts, we start with the static properties of neutron superfluidity, considering first a uniform neutron gas, and then discussing the effects of the presence of the nuclear crystal lattice. In the following, we consider superfluid hydrodynamics. We stress those points, which have been raised only recently. We consider also the important problem of the critical velocity above which superfluid flow breaks down. The interplay of superfluid flow and vortices is reviewed. The section ends with a discussion of entrainment effects. Transport phenomena are reviewed in Section 9. We present calculational methods and results for electrical and thermal conductivity, and shear viscosity of neutron star crusts. Differences between accreted and ground-state crusts, and the potential role of impurities are illustrated by examples. Finally, we discuss the very important effects of magnetic fields on transport parameters. In Section 10, we review macroscopic models of the crust, and we describe in particular a two-fluid model, which takes into account the stratification of crust layers, as well as the presence of a neutron superfluid. We show how entrainment effects between the superfluid and the charged components can be included using the variational approach developed by Brandon Carter [70]. Section 11 is devoted to a description of the wealth of neutrino emission processes associated with crusts. We limit ourselves to the basic mechanisms, which, according to existing calculations, are the most important ones at subsequent stages of neutron-star cooling.

The confrontation of theory with observations is presented in Section 12. Neutron stars are born very hot, and we briefly describe in Section 12.1 the present status of the theory of hot dense matter at subnuclear densities; this layer of the proto-neutron star will eventually become the neutron star crust. The crust is crucial for neutron star cooling, as observed by a distant observer. Namely, the crust separates the neutron star core from its surface, where the observed X-ray radiation is produced. The relation between crust physics and observations of cooling neutron stars is studied in Section 12.2. In Section 12.3 we briefly consider possible r-processes associated with the ejection and subsequent decompression of the neutron star crusts. Pulsar glitches are thought to originate in neutron star crust and glitch models are confronted with observations of glitches in pulsar timing data in Section 12.4. The asteroseismology of neutron stars from their gravitational wave radiation is discussed in Section 12.5. Due to its elasticity, the solid crust can support mountains and shear (torsional) oscillations, both associated with gravitational wave emission. The crustcore interface can be crucial for the damping of r-modes, which, if unstable, could be a promising source of gravitational waves from rotating neutron stars. Observations of oscillations in the giant flares from Soft Gamma Repeaters are confronted with models of torsional oscillations of crusts in Section 12.6. As discussed in Section 12.7, the modeling of phenomena associated with low-mass X-ray binaries (LMXB) requires a rather detailed knowledge of the physics of neutron star crusts. 
New phenomena discovered in the last decade (and some very recently) necessitate realistic physics of accreted neutron star crusts, including deep crustal heating and the correct degree of purity. All aspects of accreted crusts, relevant for soft X-ray transients, X-ray superbursts, and persistent $\mathrm{X}$-ray transients, are discussed in this section. 


\section{Plasma Parameters}

\subsection{No magnetic field}

In this section we introduce several parameters that will be used throughout this review. We follow the notations of the book by Haensel, Potekhin and Yakovlev [184].

We consider a one-component plasma model of neutron star crusts, assuming a single species of nuclei at a given density $\rho$. We restrict ourselves to matter composed of atomic nuclei immersed in a nearly ideal and uniform, strongly degenerate electron gas of number density $n_{e}$. This model is valid at $\rho>10^{5} \mathrm{~g} \mathrm{~cm}^{-3}$. A neutron gas is also present at densities greater than neutron drip density $\rho_{\mathrm{ND}} \approx 4 \times 10^{11} \mathrm{~g} \mathrm{~cm}^{-3}$.

For an ideal, fully degenerate, relativistic electron gas, the Fermi energy (using the letter F to denote quantities evaluated at the Fermi level and the letter $e$ for electrons) is given by

$$
\epsilon_{\mathrm{F} e}=m_{e}^{*} c^{2}, m_{e}^{*}=m_{e}\left(1+x_{\mathrm{r}}^{2}\right)^{1 / 2}
$$

where $x_{\mathrm{r}}$ is a dimensionless relativity parameter defined in terms of the Fermi momentum

$$
p_{\mathrm{F} e}=\hbar\left(3 \pi^{2} n_{e}\right)^{1 / 3},
$$

by

$$
x_{\mathrm{r}}=\frac{p_{\mathrm{Fe}}}{m_{e} c}
$$

and $m_{e}^{*}$ is the electron effective mass at the Fermi surface. The Fermi velocity is

$$
v_{\mathrm{F} e}=\frac{p_{\mathrm{F} e}}{m_{e}^{*}}=c \frac{x_{\mathrm{r}}}{\left(1+x_{\mathrm{r}}^{2}\right)^{1 / 2}} .
$$

Electrons are strongly degenerate for $T \ll T_{\mathrm{F} e}$, where the electron Fermi temperature is defined by

$$
T_{\mathrm{F} e}=\frac{\epsilon_{\mathrm{F} e}-m_{e} c^{2}}{k_{\mathrm{B}}}=5.93 \times 10^{9}\left(\gamma_{\mathrm{r}}-1\right) \mathrm{K},
$$

$k_{\mathrm{B}}$ is the Boltzmann constant and

$$
\gamma_{\mathrm{r}}=\frac{m_{e}^{*}}{m_{e}}=\left(1+x_{\mathrm{r}}^{2}\right)^{1 / 2}
$$

Let the mass number of nuclei be $A$ and their proton number be $Z$. The electric charge neutrality of matter implies that the number density of ions (nuclei) is

$$
n_{\mathrm{N}}=n_{\mathrm{e}} / Z
$$

For $\rho<\rho_{\mathrm{ND}}$, the quantities $n_{\mathrm{N}}$ and $A$ are related to the mass density of the crust by

$$
\rho \approx n_{\mathrm{N}} A m_{\mathrm{u}}
$$

where $m_{\mathrm{u}}$ is the atomic mass unit, $m_{\mathrm{u}}=1.6605 \times 10^{-24} \mathrm{~g}$. For $\rho>\rho_{\mathrm{ND}}$, one has to replace $A$ in Equation (8) by $A^{\prime}=A+A^{\prime \prime}$, where $A$ is the number of nucleons bound in the nucleus and $A^{\prime \prime}$ is the number of free (unbound) neutrons per ion. $A^{\prime}$ is, thus, the number of nucleons per ion. The electron relativity parameter can be expressed as

$$
x_{\mathrm{r}}=1.00884\left(\frac{\rho_{6} Z}{A^{\prime}}\right)^{1 / 3},
$$


where $\rho_{6} \equiv \rho / 10^{6} \mathrm{~g} \mathrm{~cm}^{-3}$.

The ion plasma temperature $T_{\mathrm{pi}}$ is defined, in terms of the the ion plasma frequency

$$
\omega_{\mathrm{pi}}=\left(\frac{4 \pi e^{2} n_{\mathrm{N}} Z^{2}}{A m_{\mathrm{u}}}\right)^{1 / 2}
$$

by

$$
T_{\mathrm{pi}}=\frac{\hbar \omega_{\mathrm{pi}}}{k_{\mathrm{B}}}=7.832 \times 10^{6}\left(\frac{\rho_{6}}{A^{\prime}} \frac{Z^{2}}{A}\right)^{1 / 2} \mathrm{~K} .
$$

Quantum effects for ions become very important for $T \ll T_{\mathrm{pi}}$. The electron plasma frequency is

$$
\omega_{\mathrm{p} e}=\left(\frac{4 \pi e^{2} n_{e}}{m_{e}^{*}}\right)^{1 / 2}
$$

so that the electron plasma temperature

$$
T_{\mathrm{p} e}=\frac{\hbar \omega_{\mathrm{p} e}}{k_{\mathrm{B}}}=3.300 \times 10^{8} x_{\mathrm{r}}^{3 / 2} \gamma_{\mathrm{r}}^{-1 / 2} \mathrm{~K},
$$

which can be rewritten as

$$
T_{\mathrm{p} e}=3.34 \times 10^{8} \mathrm{~K}\left(\rho_{6} \frac{Z}{A}\right)^{1 / 2} \times\left[1+1.02\left(\rho_{6} \frac{Z}{A}\right)^{2 / 3}\right]^{-1 / 4} .
$$

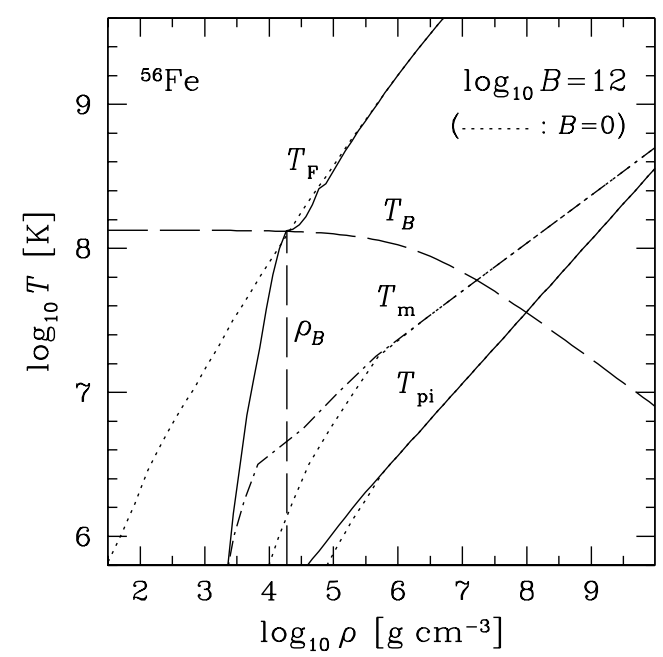

Figure 2: Different parameter domains in the $\rho-T$ plane for ${ }^{56} \mathrm{Fe}$ plasma with magnetic field $B=10^{12} \mathrm{G}$. Dash-dot line: melting temperature $T_{\mathrm{m}}$. Solid lines: $T_{\mathrm{F}}-$ Fermi temperature for the electrons (noted $T_{\mathrm{Fe}}$ in the main text); $T_{\mathrm{pi}}$ - ion plasma temperature. Long-dash lines: $T_{B}$ and $\rho_{B}$ relevant for the quantized regime of the electrons (Section 2.2); for comparison we also show, by dotted lines, $T_{\mathrm{F}}, T_{\mathrm{m}}$ and $T_{\mathrm{pi}}$ for $B=0$. For further explanation see the text. From [184].

The crystallization of a Coulomb plasma of ions occurs at the temperature

$$
T_{\mathrm{m}}=\frac{Z^{2} e^{2}}{R_{\text {cell }} k_{\mathrm{B}} \Gamma_{\mathrm{m}}} \approx 1.3 \times 10^{5} Z^{2}\left(\frac{\rho_{6}}{A^{\prime}}\right)^{1 / 3} \frac{175}{\Gamma_{\mathrm{m}}} \mathrm{K}
$$



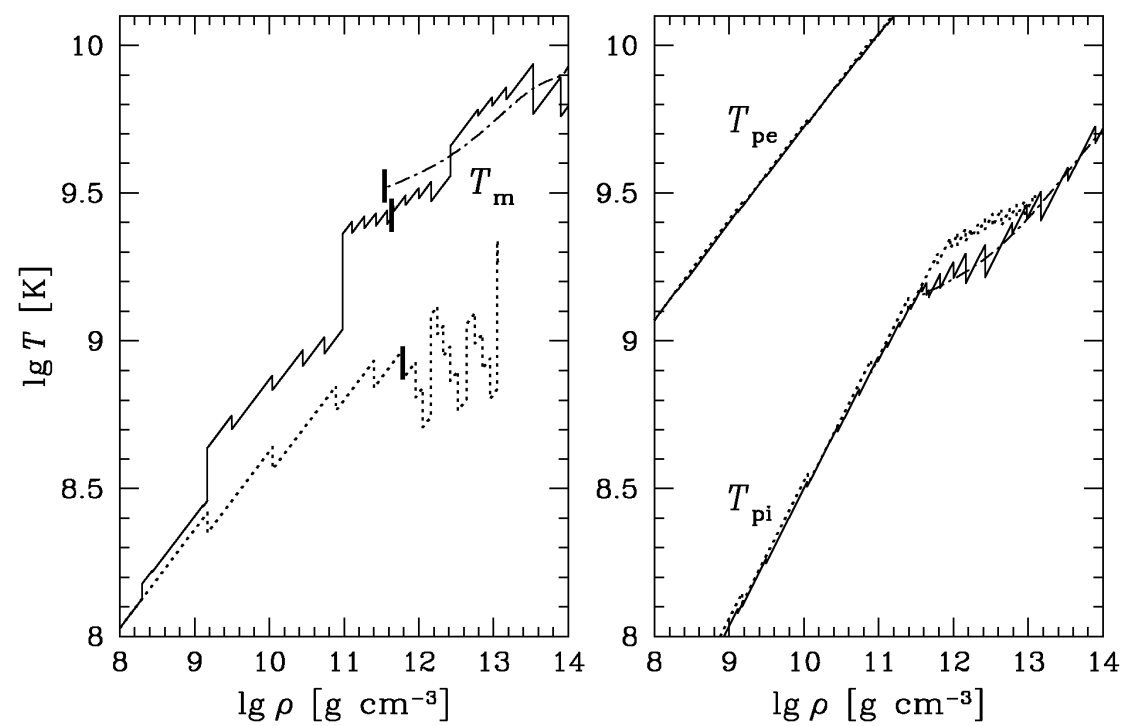

Figure 3: Left panel: melting temperature versus density. Right panel: electron and ion plasma temperature versus density. Solid lines: the ground-state composition of the crust is assumed: Haensel \& Pichon [183] for the outer crust, and Negele \& Vautherin [303] for the inner crust. Dot lines: accreted crust, as calculated by Haensel \& Zdunik [185]. Jumps result from discontinuous changes of $Z$ and $A$. Dot-dash line: results obtained for the compressible liquid drop model of Douchin \& Haensel [125] for the ground state of the inner crust; a smooth behavior (absence of jumps) results from the approximation inherent in the compressible liquid drop model. Thick vertical dashes: neutron drip point for a given crust model. Figure made by A.Y. Potekhin. 
where the ion sphere (also called the unit cell or Wigner-Seitz cell) radius is

$$
R_{\text {cell }}=\left(\frac{4 \pi n_{\mathrm{N}}}{3}\right)^{-1 / 3}
$$

For classical ions $\left(T \gtrsim T_{\mathrm{pi}}\right)$ one has $\Gamma_{\mathrm{m}} \approx 175$. For $T \lesssim T_{\mathrm{pi}}$ the zero-point quantum vibrations of ions become important and lead to crystal melting at lower $\Gamma_{\mathrm{m}}$.

\subsection{Effects of magnetic fields}

Typical pulsars have surface magnetic fields $B \sim 10^{12} \mathrm{G}$. Magnetars have much higher magnetic fields, $B \sim 10^{14}-10^{15} \mathrm{G}$. The properties of the outer envelope of neutron stars can be drastically modified by a sufficiently strong magnetic field $\boldsymbol{B}$. It is convenient to introduce the "atomic" magnetic field $B_{0}$

$$
B_{0}=\frac{m_{e}^{2} e^{3} c}{\hbar^{3}}=2.35 \times 10^{9} \mathrm{G} .
$$

It is the value of $B$ for which the electron cyclotron energy is equal to $e^{2} / a_{0}=2 \times 13.6 \mathrm{eV}\left(a_{0}\right.$ is the Bohr radius). Putting it differently, at $B=B_{0}$ the characteristic magnetic length $a_{\mathrm{m}}=(\hbar c / e B)^{1 / 2}$ equals the Bohr radius. For typical pulsars and magnetars the surface magnetic field is significantly stronger than $B_{0}$. As a result, the atomic structure at low pressure is expected to be strongly modified. The motion of electrons perpendicular to $B$ is quantized into Landau orbitals. Assuming that $\boldsymbol{B}=[0,0, B]$, the electron energy levels are given by $\epsilon_{n}\left(p_{z}\right)=c\left(m_{e}^{2} c^{2}+2 \hbar \omega_{\mathrm{ce}} m_{e} n+p_{z}^{2}\right)^{1 / 2}$, where $n$ is the Landau quantum number and $p_{z}$ the z-component of the electron momentum. The ground state Landau level $n=0$ is nondegenerate with respect to the spin (the spin is antiparallel to $\boldsymbol{B}$, with spin quantum number $s=-1$ ), while the higher levels $n>0$ are doubly degenerate $(s= \pm 1)$. The cyclotron frequency for electrons is $\omega_{\mathrm{c} e}=e B / m_{e} c$; it is 1836 times larger than for protons. The Coulomb binding of electrons by the atomic nucleus is significantly less effective along $\boldsymbol{B}$, while in the plane perpendicular to $\boldsymbol{B}$ the electron motion is confined to the $n=0$ Landau level. Therefore atoms get a cylindrical shape and can form linear chains along $\boldsymbol{B}$. The attraction between these chains can lead to a phase transition into a "magnetically condensed" phase (for a recent review on this topic, see Medin \& Lai [287]). The density of the condensed phase at zero pressure $P=0$ (i.e. at the stellar surface) and zero temperature $T=0$ is

$$
\rho_{\mathrm{s}} \simeq 4.4 \times 10^{3} \frac{A}{56}\left(\frac{Z}{26}\right)^{-3 / 5} B_{12}^{6 / 5} \mathrm{~g} \mathrm{~cm}^{-3},
$$

where $B_{12}=B / 10^{12} \mathrm{G}$. For each element, there is a critical temperature at given $B$, below which a magnetically condensed phase exists at $P=0$. The values of $T_{\text {crit }}$ at several $B$ for ${ }^{56} \mathrm{Fe},{ }^{12} \mathrm{C}$, and ${ }^{4} \mathrm{He}$ are given in Table 1.

Table 1: Critical temperature (in K) below which a condensed phase exists at $P=0$, for several magnetic field strength $B_{12}=B / 10^{12} \mathrm{G}$ and matter composition. From [287].

\begin{tabular}{c|ccc}
\hline$B_{12}=$ & 10 & 100 & 1000 \\
\hline${ }^{56} \mathrm{Fe}$ & $7 \times 10^{5}$ & $3 \times 10^{6}$ & $2 \times 10^{7}$ \\
${ }^{12} \mathrm{C}$ & $3 \times 10^{5}$ & $3 \times 10^{6}$ & $2 \times 10^{7}$ \\
${ }^{4} \mathrm{He}$ & $3 \times 10^{5}$ & $2 \times 10^{6}$ & $9 \times 10^{7}$ \\
\hline
\end{tabular}

We now briefly consider the effects of the magnetic field on plasma properties at finite pressure $P>0$. The magnetic field strongly quantizes the motion of electrons, if it confines most of them 
to the ground Landau state $n=0$. Parameters relevant to a strong quantization regime are

$$
T_{\mathrm{ce}}=\frac{\hbar \omega_{\mathrm{ce}}}{k_{\mathrm{B}}} \approx 1.343 \times 10^{8} B_{12} \mathrm{~K}, \quad \rho_{B}=7.045 \times 10^{3} \frac{A^{\prime}}{Z} B_{12}^{3 / 2} \mathrm{~g} \mathrm{~cm}^{-3},
$$

and

$$
T_{B}=T_{\mathrm{ce}} \text { if } \rho<\rho_{B}, \quad \text { or } \quad T_{B}=T_{\mathrm{ce}} / \sqrt{1+x_{\mathrm{r}}^{2}} \text { if } \rho>\rho_{B} .
$$

The field $B$ is strongly quantizing if $\rho<\rho_{B}$ and $T \ll T_{\text {ce }}$. On the contrary, a magnetic field is called weakly quantizing if many Landau levels are occupied, but still $T \ll T_{B}$. Finally, $B$ is nonquantizing if $T \gg T_{B}$. The temperature $T_{B}$ and density $\rho_{B}$ are shown in Figure 2.

The magnetic field can strongly modify transport properties (Section 9.5) and neutrino emission (Section 11.7). Its effect on the equation of state is significant only if it is strongly quantizing (see Section 6.4). 


\section{The Ground State Structure of Neutron Star Crusts}

According to the cold catalyzed matter hypothesis, the matter inside cold non-accreting neutron stars is assumed to be in complete thermodynamic equilibrium with respect to all interactions at zero temperature and is therefore supposed to be in its ground state with the lowest possible energy. The validity of this assumption is discussed in Section 3.4.

The ground state structure of a neutron star crust is sketched in Figure 4 . The outer crust (Section 3.1) consists of a body-centered cubic lattice of iron ${ }^{56} \mathrm{Fe}$. At $\rho \sim 10^{4} \mathrm{~g} \mathrm{~cm}^{-3}$ the atoms are fully ionized owing to the high density. At densities above $10^{7} \mathrm{~g} \mathrm{~cm}^{-3}$, the composition of the nuclei becomes more neutron rich as a result of electron captures. The inner crust (Section 3.2), which extends from $\rho_{\mathrm{ND}} \simeq 4 \times 10^{11} \mathrm{~g} \mathrm{~cm}^{-3}$ to $\sim \rho_{0} / 3 \simeq 10^{14} \mathrm{~g} \mathrm{~cm}^{-3}$, is characterized by the presence of free neutrons, which may condense into a superfluid phase in some layers (see Section 8). At the bottom of the crust, some calculations predict various "pasta" phases of non-spherical nuclei, such as slabs or cylinders as discussed in Section 3.3.

The ground state of a neutron star crust is obtained by minimizing the total energy density $\varepsilon_{\text {tot }}$ for a given baryon density $n_{\mathrm{b}}$ under the assumption of $\beta$-equilibrium and electric charge neutrality. For simplicity, the crust is assumed to be formed of a perfect crystal with a single nuclear species at lattice sites (see Jog \& Smith [221] and references therein for the possibility of heteronuclear compounds).

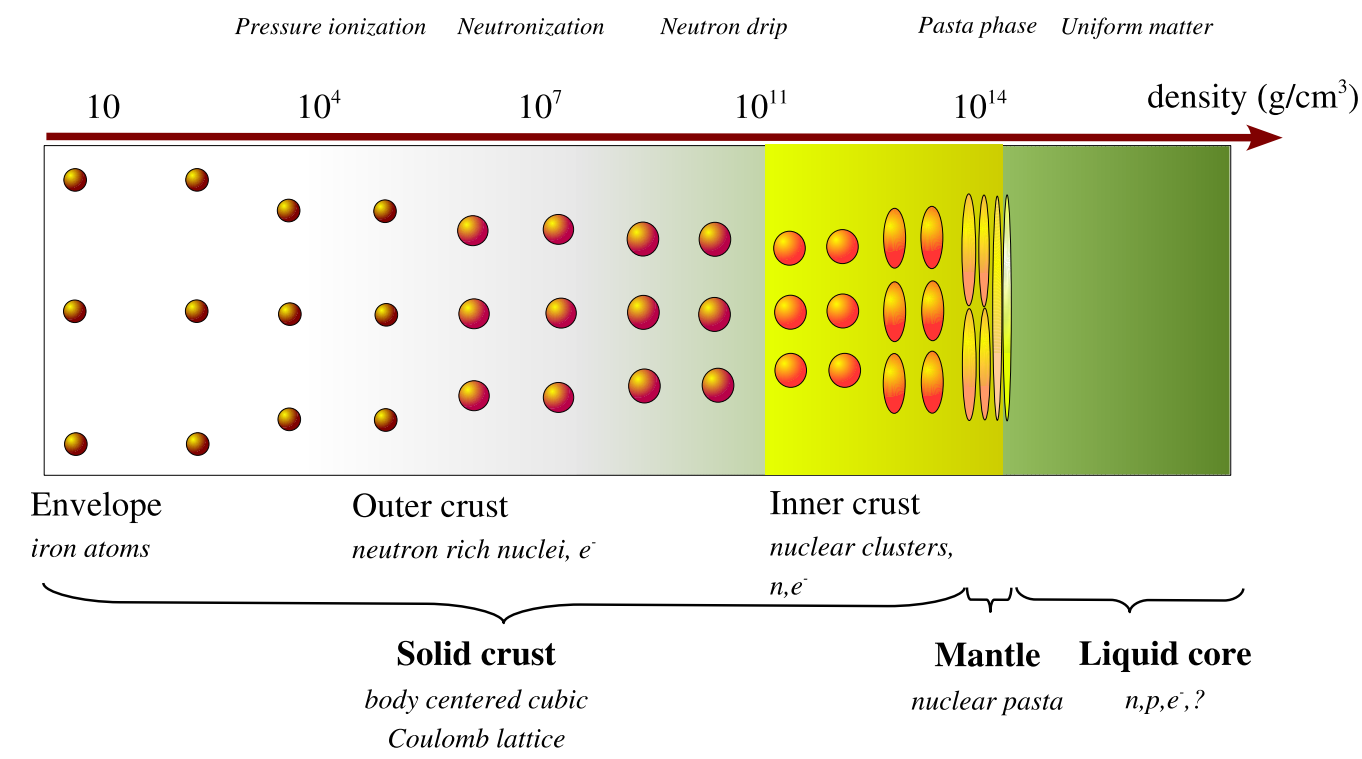

Figure 4: Schematic picture of the ground state structure of neutron stars along the density axis. Note that the main part of this figure represents the solid crust since it covers about 14 orders of magnitude in densities.

\subsection{Structure of the outer crust}

Matter at densities below neutron drip $\rho_{\mathrm{ND}}$ is not only relevant for neutron star crusts but also for white dwarfs. Following the classical paper of Baym, Pethick and Sutherland [42], the total energy density in a given layer can be written as

$$
\varepsilon_{\mathrm{tot}}=n_{\mathrm{N}} E\{A, Z\}+\varepsilon_{e}+\varepsilon_{L}
$$


where $n_{\mathrm{N}}$ is the number density of nuclei, $E\{A, Z\}$ is the energy of a nucleus with $Z$ protons and $A-Z$ neutrons, $\varepsilon_{e}$ is the electron kinetic energy density and $\varepsilon_{L}$ is the lattice energy density, which accounts for the electron-electron, electron-ion and ion-ion Coulomb interactions.

In dense, cold, neutron star crust, electron-charge-screening effects are negligible and the electron density is essentially uniform [418]. The reason is that the electron Thomas-Fermi screening length is larger than the lattice spacing [326]. Charge screening effects are much more important in neutron star matter with large proton fractions, such as encountered in supernovae and newly born hot neutron stars [282]. At densities $\rho \gg 10 A Z \mathrm{~g} \mathrm{~cm}^{-3}\left(\sim 10^{4} \mathrm{~g} \mathrm{~cm}^{-3}\right.$ for iron), the electrons can be treated as a quasi-ideal Fermi gas so that

$$
\varepsilon_{e}=\frac{m_{e}^{4} c^{5}}{8 \pi^{2} \hbar^{3}}\left(x_{r}\left(2 x_{r}^{2}+1\right) \sqrt{x_{r}^{2}+1}-\ln \left\{x_{r}+\sqrt{x_{r}^{2}+1}\right\}\right),
$$

with $x_{r}$ defined by Equation (3).

The lattice energy density can be estimated from the Wigner-Seitz approximation illustrated on Figure 5. The crust is decomposed into a set of independent spheres centered around each nucleus, with a radius $R_{\text {cell }}$ defined by Equation (16). Each sphere is electrically neutral and therefore contains $Z$ protons and $Z$ electrons. The lattice energy density is then given by the density of nuclei times the Coulomb energy of one such sphere (excluding the Coulomb energy of the nucleus, which is already taken into account in $E\{A, Z\}$ ). Assuming point-like nuclei since the lattice spacing is much larger than the size of the nuclei ${ }^{1}$, the lattice energy can be expressed as (see, for instance, Shapiro \& Teukolsky [373], p30-31)

$$
\varepsilon_{L}=-\frac{9}{10}\left(\frac{4 \pi}{3}\right)^{1 / 3} Z^{2 / 3} e^{2} n_{e}^{4 / 3}
$$

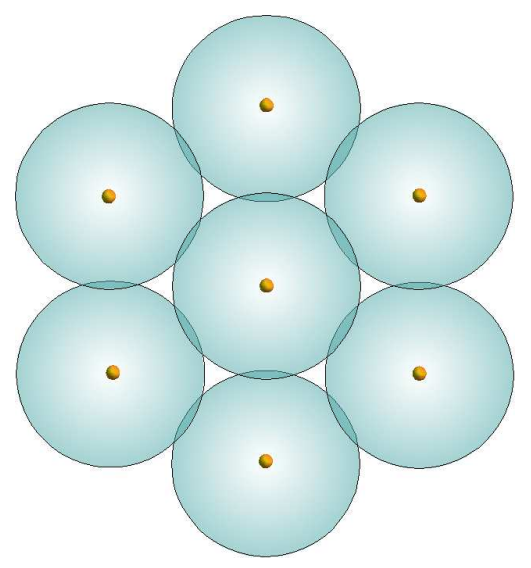

Figure 5: In the Wigner-Seitz approximation the crystal (represented here as a two-dimensional hexagonal lattice) is decomposed into independent identical spheres, centered around each site of the lattice. The radius of the sphere is chosen so that the volume of the sphere is equal to $1 / n_{\mathrm{N}}$, where $n_{\mathrm{N}}$ is the density of lattice sites (ions).

An exact calculation of the lattice energy for cubic lattices yields similar expressions except for the factor $9 / 10$, which is replaced by 0.89593 and 0.89588 for body-centered and face-centered cubic-lattices, respectively (we exclude simple cubic lattices since they are generally unstable; note

\footnotetext{
${ }^{1}$ Lattice energy including finite size effects is given by Equation (41). Even at the bottom of the outer crust finite size effects represent a small correction to the lattice energy, less than $1 \%$.
} 
that polonium is the only known element on Earth with such a crystal structure under normal conditions [258]). This shows that the equilibrium structure of the crust is expected to be a body-centered cubic lattice, since this gives the smallest lattice energy. Other lattice types, such as hexagonal closed packed for instance, might be realized in neutron star crusts. Nevertheless, the study of Kohanoff and Hansen [242] suggests that such noncubic lattices may occur only at small densities, meaning that $r_{e} \sim a_{0}$, while in the crust $r_{e} \ll a_{0}$, where $r_{e} \equiv\left(3 / 4 \pi n_{e}\right)^{1 / 3}$ and $a_{0}=\hbar^{2} / m_{e} e^{2}$ is the Bohr radius. Equation (23) shows that the lattice energy is negative and therefore reduces the total Coulomb energy. The lattice contribution to the total energy density is small but large enough to affect the equilibrium structure of the crust by favoring large nuclei. Corrections due to electron-exchange interactions, electron polarization and quantum zero point motion of the ions are discussed in the book by Haensel, Potekhin and Yakovlev [184].

The main physical input is the energy $E\{A, Z\}$, which has been experimentally measured for more than 2000 known nuclei [25]. Nevertheless, this quantity has not been measured yet for the very neutron rich nuclei that could be present in the dense layers of the crust and has therefore to be calculated. The most accurate theoretical microscopic nuclear mass tables, using self-consistent mean field methods, have been calculated by the Brussels group and are available on line [211].

According to the first law of thermodynamics, the total pressure $P$ is given by

$$
P=n_{\mathrm{b}}^{2} \frac{\mathrm{d}}{\mathrm{d} n_{\mathrm{b}}}\left(\frac{\varepsilon_{\mathrm{tot}}}{n_{\mathrm{b}}}\right) .
$$

Using Equation (21), the total pressure can be expressed as

$$
P=P_{e}+\frac{1}{3} \varepsilon_{L}
$$

The electron pressure $P_{e}$ is defined by

$$
P_{e}=\varepsilon_{e}-\mu_{e} n_{e},
$$

where the electron chemical potential $\mu_{e}$ is simply given by the electron Fermi energy $\epsilon_{\mathrm{F} e}$, Equation (1), and $p_{\mathrm{Fe}}$, the electron Fermi momentum given by Equation (2). The electrons make the dominant contribution to the total pressure in the outer crust.

The structure of the ground state crust is determined by minimizing the total energy density $\varepsilon_{\text {tot }}$ for a given baryon density $n_{\mathrm{b}}=A n_{\mathrm{N}}$ imposing charge neutrality, $n_{p}=n_{e}$. However $n_{\mathrm{b}}$ (or the average mass density $\rho$ ) can suffer jumps at some values of the pressure. The pressure, on the contrary, should be continuous and monotonically increasing with increasing depth below the stellar surface. Therefore we will look for a ground state at $T=0$ and at a fixed $P$. This corresponds to minimization of the Gibbs free energy per nucleon, $g(P)=\left(\varepsilon_{\mathrm{tot}}+P\right) / n_{\mathrm{b}}$, under the condition of electric charge neutrality. For a completely-ionized one-component plasma, one constructs a table $g(P ; A, Z)$ and then finds an absolute minimum in the $(A, Z)$ plane. The procedure, based on the classical paper of Baym, Pethick and Sutherland [42], is described in detail in the book by Haensel, Potekhin and Yakovlev [184]. Every time that the ground state shifts to a new nucleus with a smaller proton fraction, $(A, Z) \longrightarrow\left(A^{\prime}, Z^{\prime}\right)$, there is a few percent jump of density at the $(A, Z)$ and $\left(A^{\prime}, Z^{\prime}\right)$ shell interface,

$$
\frac{\Delta n_{\mathrm{b}}}{n_{\mathrm{b}}} \approx \frac{\Delta \rho}{\rho} \approx \frac{Z}{A} \frac{A^{\prime}}{Z^{\prime}}-1
$$

which stems from a strict continuity of the pressure. It should be stressed that these density discontinuities are the direct consequence of the one-component plasma approximation. Jog \& Smith [221] have shown that the transition between two adjacent layers with a single nuclear species is much smoother due to the existence of mixed lattices. In particular, they have found

Living Reviews in Relativity

http://www. livingreviews . org//rr-2008-10 
that between a layer with a pure body-centered cubic (bcc) lattice of $(Z, A)$ nuclei (lower density) and a layer with a pure bcc lattice of $\left(Z^{\prime}, A^{\prime}\right)$ nuclei, a bcc lattice with $(Z, A)$ nuclei at the corners of the conventional cube and $\left(Z^{\prime}, A^{\prime}\right)$ at the center is energetically favored.

The structure of the crust is completely determined by the experimental nuclear data up to a density of the order $\rho \sim 6 \times 10^{10} \mathrm{~g} \mathrm{~cm}^{-3}$. At higher densities the nuclei are so neutron rich that they have not yet been experimentally studied, and the energy $E\{A, Z\}$ must be extrapolated. Consequently the composition of the nuclei in these dense layers is model dependent. Nevertheless most models show the predominance of nuclei with the magic neutron numbers $N=50,82$, thus revealing the crucial role played by the quantum shell effects. The structure of the outer crust is shown in Table 2 for one particularly representative recent model. Up-to-date theoretical mass tables are available online [211].

Table 2: Sequence of nuclei in the ground state of the outer crust of neutron star calculated by Rüster et al. [357] using experimental nuclear data (upper part), and the theoretical mass table of the Skyrme model BSk8 (lower part).

\begin{tabular}{ccccc}
\hline$\rho_{\max }\left[\mathrm{g} \mathrm{cm}^{-3}\right]$ & Element & $Z$ & $N$ & $R_{\text {cell }}[\mathrm{fm}]$ \\
\hline $8.02 \times 10^{6}$ & ${ }^{56} \mathrm{Fe}$ & 26 & 30 & 1404.05 \\
$2.71 \times 10^{8}$ & ${ }^{62} \mathrm{Ni}$ & 28 & 34 & 449.48 \\
$1.33 \times 10^{9}$ & ${ }^{64} \mathrm{Ni}$ & 28 & 36 & 266.97 \\
$1.50 \times 10^{9}$ & ${ }^{66} \mathrm{Ni}$ & 28 & 38 & 259.26 \\
$3.09 \times 10^{9}$ & ${ }^{86} \mathrm{Kr}$ & 36 & 50 & 222.66 \\
$1.06 \times 10^{10}$ & ${ }^{84} \mathrm{Se}$ & 34 & 50 & 146.56 \\
$2.79 \times 10^{10}$ & ${ }^{82} \mathrm{Ge}$ & 32 & 50 & 105.23 \\
$6.07 \times 10^{10}$ & ${ }^{80} \mathrm{Zn}$ & 30 & 50 & 80.58 \\
\hline $8.46 \times 10^{10}$ & ${ }^{82} \mathrm{Zn}$ & 30 & 52 & 72.77 \\
$9.67 \times 10^{10}$ & ${ }^{128} \mathrm{Pd}$ & 46 & 82 & 80.77 \\
$1.47 \times 10^{11}$ & ${ }^{126} \mathrm{Ru}$ & 44 & 82 & 69.81 \\
$2.11 \times 10^{11}$ & ${ }^{124} \mathrm{Mo}$ & 42 & 82 & 61.71 \\
$2.89 \times 10^{11}$ & ${ }^{122} \mathrm{Zr}$ & 40 & 82 & 55.22 \\
$3.97 \times 10^{11}$ & ${ }^{120} \mathrm{Sr}$ & 38 & 82 & 49.37 \\
$4.27 \times 10^{11}$ & ${ }^{118} \mathrm{Kr}$ & 36 & 82 & 47.92 \\
\hline
\end{tabular}

\subsection{Structure of the inner crust}

With increasing density, the ground-state value of $Z / A$ decreases and neutrons become less and less bound. Let us define the "net neutron chemical potential" of a neutron in a nucleus

$$
\mu_{n}^{\prime} \equiv \mu_{n}-m_{n} c^{2}=\left(\frac{\partial E\{A, Z\}}{\partial N}\right)_{Z}-m_{n} c^{2}
$$

As long as $\mu_{n}^{\prime}<0$, all neutrons are bound within nuclei. The neutron drip point corresponds to $\mu_{n}^{\prime}=0$; beyond this point neutrons "drip out of nuclei", i.e. they begin to fill states in the continuous part of the energy spectrum. We can roughly localize the neutron drip point using the approximate mass formula for $E^{\prime}\{A, Z\}=E\{A, Z\}-A m c^{2}$, where, for simplicity, we neglect the neutron-proton mass difference, putting $m_{n} \approx m_{p} \approx m=939 \mathrm{MeV} / c^{2}$. Neglecting surface and Coulomb terms, we have

$$
E^{\prime}\{A, Z\} \approx A\left(E_{\mathrm{vol}}+E_{\mathrm{sym}} \delta^{2}\right)
$$


where $\delta \equiv(N-Z) / A$, and $E_{\mathrm{vol}}$ and $E_{\mathrm{sym}}$ are nuclear volume and symmetry energies, respectively. Experimentally, $E_{\mathrm{vol}} \simeq-16 \mathrm{MeV}$ and $E_{\mathrm{sym}} \simeq 32 \mathrm{MeV}$. Using Equation (29), we can easily show that the value of $\delta$ at which $\mu_{n}^{\prime}=0$ is

$$
\delta_{\mathrm{ND}}=\sqrt{1-E_{\mathrm{vol}} / E_{\mathrm{sym}}}-1 .
$$

Using experimental values of $E_{\mathrm{vol}}$ and $E_{\mathrm{sym}}$, we find $\delta_{\mathrm{ND}}=0.225$.

Neglecting neutron-proton mass difference, the beta equilibrium condition

$$
p+e \rightarrow n+\nu_{e}
$$

reads

$$
\mu_{e}=\mu_{n}-\mu_{p} \approx 4 E_{\mathrm{sym}} \delta
$$

At the density under consideration, electrons are ultrarelativistic, so that $\mu_{e} \simeq 5.16\left(\rho_{9} Z / A\right)^{1 / 3} \mathrm{MeV}$, where $\rho_{9}=\rho / 10^{9} \mathrm{~g} \mathrm{~cm}^{-3}$. It is now easy to show that the neutron drip density is roughly $\rho_{\mathrm{ND}} \approx$ $2 \times 10^{11} \mathrm{~g} \mathrm{~cm}^{-3}$, which is about half of the value obtained in complete calculations [42, 183, 357]. For $\rho>\rho_{\mathrm{ND}} \approx 4 \times 10^{11} \mathrm{~g} \mathrm{~cm}^{-3}$ a fraction of neutrons thus forms a gas outside the nuclei.

The inner crust of a neutron star is a unique system, which is not accessible in the laboratory due to the presence of this neutron gas. In the following we shall thus refer to the "nuclei" in the inner crust as "clusters" in order to emphasize these peculiarities. The description of the crust beyond neutron drip therefore relies on theoretical models only. Many-body calculations starting from the realistic nucleon-nucleon interaction are out of reach at present due to the presence of spatial inhomogeneities of nuclear matter. Even in the simpler case of homogeneous nuclear matter, these calculations are complicated by the fact that nucleons are strongly interacting via two-body, as well as three-body, forces, which contain about twenty different operators. As a result, the inner crust of a neutron star has been studied with phenomenological models. Most of the calculations carried out in the inner crust rely on purely classical (compressible liquid drop) and semi-classical models (Thomas-Fermi approximation and its extensions). The state-of-the-art calculations performed so far are based on self-consistent mean field methods, which have been very successful in predicting the properties of heavy laboratory nuclei.

\subsubsection{Liquid drop models}

We will present in detail the liquid drop model because this approach provides very useful insight despite its simplicity. As in Section 3.1, we first start by writing the total energy density including the contribution $\varepsilon_{n}$ of the neutron gas

$$
\varepsilon_{\mathrm{tot}}=n_{\mathrm{N}} E\{A, Z\}+\varepsilon_{e}+\varepsilon_{L}+\varepsilon_{n} .
$$

The nuclear clusters are treated as liquid drops of nuclear matter whose energy can be decomposed into volume, surface and Coulomb terms

$$
E\{A, Z\}=E_{\mathrm{N}, \mathrm{vol}}+E_{\mathrm{N}, \mathrm{surf}}+E_{\mathrm{N}, \mathrm{Coul}} .
$$

In the simplest version, the drop is supposed to be incompressible with a density on the order of $\rho_{0}$ corresponding to the density inside heavy nuclei. This implies that the volume and surface terms in Equation (34) are proportional to $A$ and $A^{2 / 3}$, respectively. Each contribution to the energy, Equation (34), can then be parameterized in terms of the numbers $A$ and $Z$. The parameters are adjusted to the known experimental masses of nuclei, with $Z / A \sim 0.5$. The first models of neutron star crust were based on such semi-empirical mass formulae, see for instance the book by Haensel, Potekhin and Yakovlev [184]. However, such formulae can not be reliably extrapolated to the

Living Reviews in Relativity

http://www. livingreviews.org/Irr-2008-10 
neutron rich nuclear clusters in neutron star crusts, where $Z / A$ varies from $\sim 0.3$ at the neutron drip threshold to $\sim 0.1$ at the bottom of the crust. Besides, the presence of the neutron liquid has a profound effect on the clusters. First, it reduces the surface energy of the clusters as compared to that of isolated nuclei. Second, it exerts a pressure on the clusters. A major breakthrough was reached by Baym, Bethe and Pethick [39], who applied a compressible liquid-drop model, which included the results of microscopic many-body calculations, to describe consistently both the nucleons in the clusters and the "free" neutrons. The energy Equation (34) of the cluster then depends not only on $A$ and $Z$ but on a few additional parameters, such as, for instance, the size of the cluster and the density of the neutrons and protons inside it.

The volume contribution in Equation (34) is given by

$$
E_{\mathrm{N}, \mathrm{vol}}=\varepsilon\left\{n_{n \mathrm{i}}, n_{p \mathrm{i}}\right\} \mathcal{V}_{\mathrm{N}},
$$

where $\varepsilon\left\{n_{n}, n_{p}\right\}$ is the energy density of homogeneous nuclear matter and $n_{n \mathrm{i}}$ and $n_{p \mathrm{i}}$ are respectively the neutron and proton densities inside the clusters. $\mathcal{V}_{\mathrm{N}}$ is the volume of the cluster. For consistency the energy density of the surrounding neutron gas is expressed in terms of the same function $\varepsilon\left\{n_{n}, n_{p}\right\}$ as

$$
\varepsilon_{n}=\varepsilon\left\{n_{n \mathrm{o}}, 0\right\}(1-w),
$$

where $n_{n o}$ is the number density of free neutrons outside the clusters and

$$
w=\frac{\mathcal{V}_{\mathrm{N}}}{\mathcal{V}_{\text {cell }}}=\left(\frac{r_{p}}{R_{\text {cell }}}\right)^{3}
$$

is the volume fraction of the cluster. Let us define the surface thermodynamic potential per unit area $\sigma$ and the chemical potential $\mu_{n \mathrm{~s}}$ of neutrons adsorbed on the surface of the drop (forming a neutron skin) by

$$
\sigma=\left.\frac{\partial E_{\mathrm{N}, \text { surf }}}{\partial \mathcal{A}}\right|_{N_{\mathrm{s}}}, \quad \mu_{n \mathrm{~s}}=\left.\frac{\partial E_{\mathrm{N}, \mathrm{surf}}}{\partial N_{\mathrm{s}}}\right|_{\mathcal{A}},
$$

where $\mathcal{A}$ is the surface area of the cluster and $N_{\mathrm{s}}$ is the number of adsorbed neutrons. Since energy is an extensive thermodynamic variable, it follows from Euler's theorem about homogeneous functions that

$$
E_{\mathrm{N}, \mathrm{surf}}=\sigma \mathcal{A}+N_{\mathrm{s}} \mu_{n \mathrm{~s}} .
$$

The Coulomb energy of a uniformly-charged spherical drop of radius $r_{p}$ is given by

$$
E_{\mathrm{N}, \mathrm{Coul}}=\frac{3}{5} \frac{Z^{2} e^{2}}{r_{p}}
$$

Corrections due to the diffuseness of the cluster surface and due to quantum exchange can be found, for instance, in reference [277]. The electron energy density is approximately given by Equation (22). In the Wigner-Seitz approximation, assuming uniformly-charged spherical clusters of radius $r_{p}$, the lattice energy is given by [39]

$$
\varepsilon_{L}=-\frac{9}{10}\left(\frac{4 \pi}{3}\right)^{1 / 3} Z^{2 / 3} e^{2} n_{e}^{4 / 3}\left(1-\frac{1}{3} w^{2 / 3}\right) .
$$

The physical input of the liquid drop model is the energy density of homogeneous nuclear matter $\varepsilon\left\{n_{n}, n_{p}\right\}$, the surface potential $\sigma$ and the chemical potential $\mu_{n \mathrm{~s}}$. For consistency, these ingredients should be calculated from the same microscopic nuclear model. The surface properties are usually determined by considering two semi-infinite phases in equilibrium (nucleons in clusters and free 
neutrons outside) separated by a plane interface (for curvature corrections, see, for instance, [273, 126]). In this approximation, the surface energy is proportional to the surface area

$$
E_{\mathrm{N}, \text { surf }}=\left(\sigma_{\mathrm{s}}+s_{n}\left(n_{n \mathrm{i}}-n_{n \mathrm{o}}\right) \mu_{n \mathrm{~s}}\right) \mathcal{A},
$$

where $\sigma_{\mathrm{s}}$ is the surface tension and $s_{n}$ the neutron skin thickness. Unlike the surface area $\mathcal{A}$, the proportionality coefficient does not depend on the actual shape of the nuclear clusters.

The structure of the inner crust is determined by minimizing the total energy density $\varepsilon_{\text {tot }}$ for a given baryon density $n_{\mathrm{b}}$ imposing electric charge neutrality $n_{p}=n_{e}$. The conditions of equilibrium are obtained by taking the partial derivative of the energy density $\varepsilon_{\text {tot }}$ with respect to the free parameters of the model. In the following we shall neglect curvature corrections to the surface energy. In this approximation, the surface potential $\sigma_{\mathrm{s}}$ is independent of the shape and size of the drop. The variation of the surface tension with the neutron excess is illustrated in Figure 6.

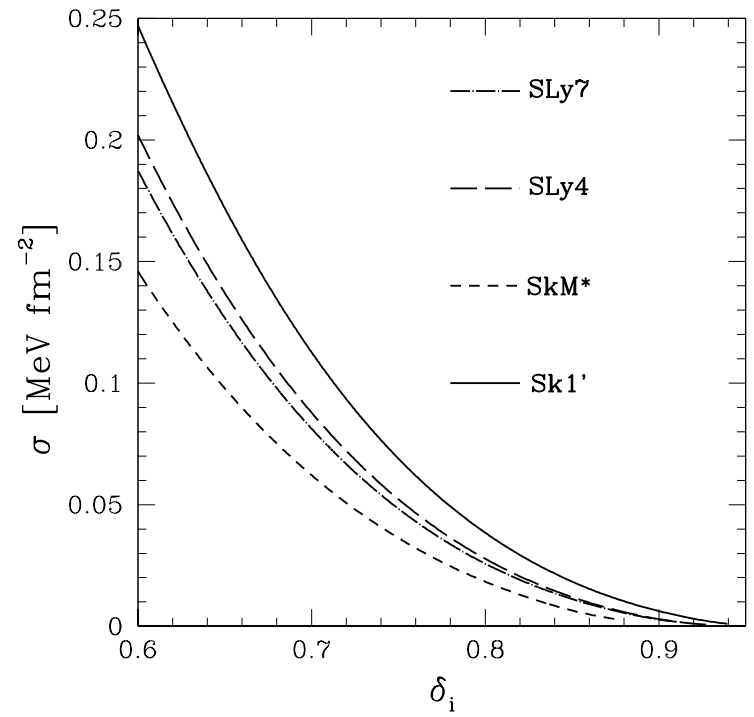

Figure 6: Surface tension of the nuclei in neutron star crusts versus neutron excess parameter $\delta_{\mathrm{i}}$ inside the nuclear cluster, in the plane interface approximation with different Skyrme models [123].

The conditions of chemical equilibrium are

$$
\begin{gathered}
\mu_{n \mathrm{i}}^{\mathrm{bulk}}=\mu_{n \mathrm{o}}^{\mathrm{bulk}}=\mu_{n \mathrm{~s}}, \\
\mu_{n \mathrm{i}}^{\mathrm{bulk}}-\mu_{p \mathrm{i}}^{\mathrm{bulk}}-\mu_{e}=\frac{8 \pi}{5} e^{2} n_{p \mathrm{i}} r_{p}^{2} f_{3}\{w\},
\end{gathered}
$$

where

$$
\mu_{X}^{\text {bulk }} \equiv \frac{\partial \varepsilon}{\partial n_{X}}
$$

and $X=n \mathrm{i}, n \mathrm{o}, n \mathrm{~s}, \ldots$

$$
f_{3}\{w\} \equiv 1-\frac{3}{2} w^{1 / 3}+\frac{1}{2} w
$$


The mechanical equilibrium of the cluster is expressed by

$$
P_{\mathrm{i}}^{\mathrm{bulk}}-P_{\mathrm{o}}^{\mathrm{bulk}}=\frac{2 \sigma_{\mathrm{s}}}{r_{p}}-\frac{4 \pi}{15} e^{2} n_{p \mathrm{i}}^{2} r_{p}^{2}(1-w),
$$

which can be easily recognized as a generalization of Laplace's law for an isolated drop. We have introduced bulk nuclear pressures by

$$
P_{X}^{\mathrm{bulk}} \equiv n_{X}^{2} \frac{\partial\left(\varepsilon / n_{X}\right)}{\partial n_{X}}=n_{X} \mu_{X}^{\mathrm{bulk}}-\varepsilon .
$$

Note that $P_{\mathrm{i}}^{\text {bulk }}$ means the total pressure inside the drop, $P_{\mathrm{i}}^{\mathrm{bulk}} \equiv P_{n \mathrm{i}}^{\mathrm{bulk}}+P_{p \mathrm{i}}^{\mathrm{bulk}}$. The last term in Equation (47) comes from the pressure due to Coulomb forces between protons inside the cluster and between clusters of the lattice.

The mechanical equilibrium of the crystal lattice can be expressed as

$$
E_{\mathrm{N}, \mathrm{surf}}=2 E_{\mathrm{Coul}}
$$

where $E_{\text {Coul }}$ is the total Coulomb energy

$$
E_{\text {Coul }}=E_{\mathrm{N}, \text { Coul }}+\mathcal{V}_{\text {cell }} \varepsilon_{L}
$$

Relation (49) is referred to as a "virial" theorem [39].

Combining Equations (40) and (41), the total Coulomb energy can be written as

$$
E_{\text {Coul }}=E_{\mathrm{N}, \text { Coul }} f_{3}\{w\},
$$

where the dimensionless function $f_{3}\{w\}$ is given by Equation (46). Let us emphasize that the above equilibrium conditions are only valid if nuclear surface curvature corrections are neglected.

Equation (49) shows that the equilibrium composition of the cluster is a result of the competition between Coulomb effects, which favor small clusters, and surface effects, which favor large clusters. This also shows that the lattice energy is very important for determining the equilibrium shape of the cluster, especially at the bottom of the crust, where the size of the cluster is of the same order as the lattice spacing. Even at the neutron drip, the lattice energy reduces the total Coulomb energy by about $15 \%$.

The structure of the inner crust, as calculated from a compressible liquid drop model by Douchin \& Haensel [125], is illustrated in Figures 7 and 8. One remarkable feature, which is confirmed by more realistic models, is that the number $Z \sim 40$ of protons in the clusters is almost constant throughout the inner crust. It can also be seen that, as the density increases, the clusters get closer and closer, while their size $r_{p}$ varies very little. Let us also notice that at the bottom of the crust the number $N_{\mathrm{s}}$ of neutrons, adsorbed on the surface of the clusters, decreases with increasing density, because the properties of the matter inside and outside the clusters become more and more alike. The results of different liquid drop models are compared in Figure 9.

The liquid drop model is very instructive for understanding the contribution of different physical effects to the structure of the crust. However this model is purely classical and consequently neglects quantum effects. Besides, the assumption of clusters with a sharp cut surface is questionable, especially in the high density layers where the nuclei are very neutron rich.

\subsubsection{Semi-classical models}

Semi-classical models have been widely applied to study the structure of neutron star crusts. These models assume that the number of particles is so large that the quantum numbers describing the system vary continuously and instead of wave functions one can use the number densities of the 


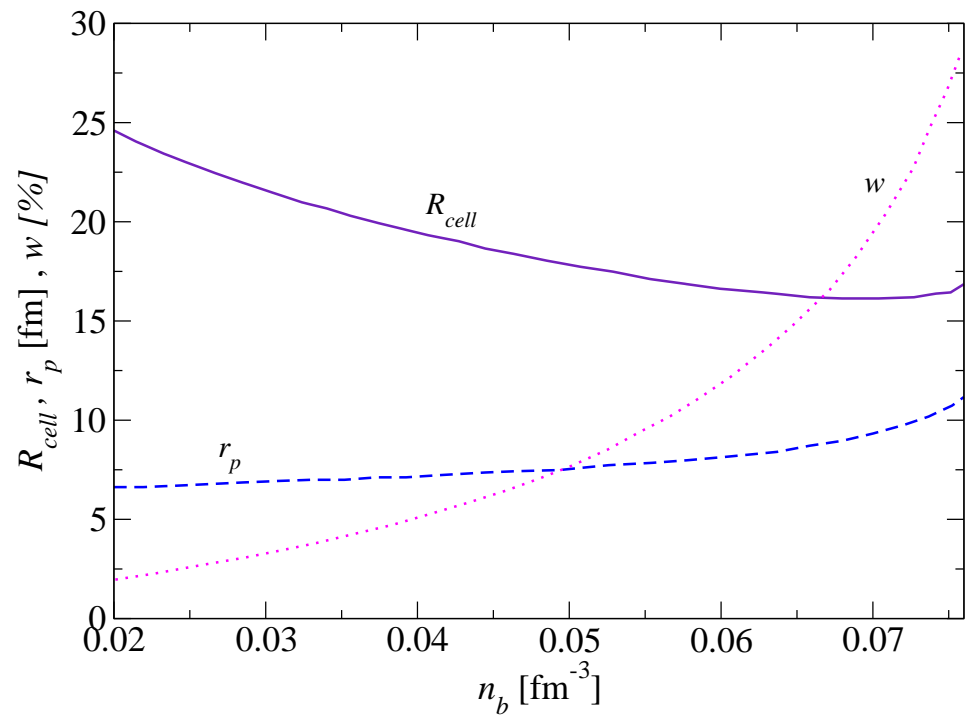

Figure 7: Structure of the ground state of the inner crust. Radius $R_{\text {cell }}$ of the Wigner-Seitz cell, proton radius $r_{p}$ of spherical nuclei, and fraction $w$ of volume filled by nuclear clusters (in percent), versus average baryon number density $n_{\mathrm{b}}$ as calculated by Douchin \& Haensel [124].

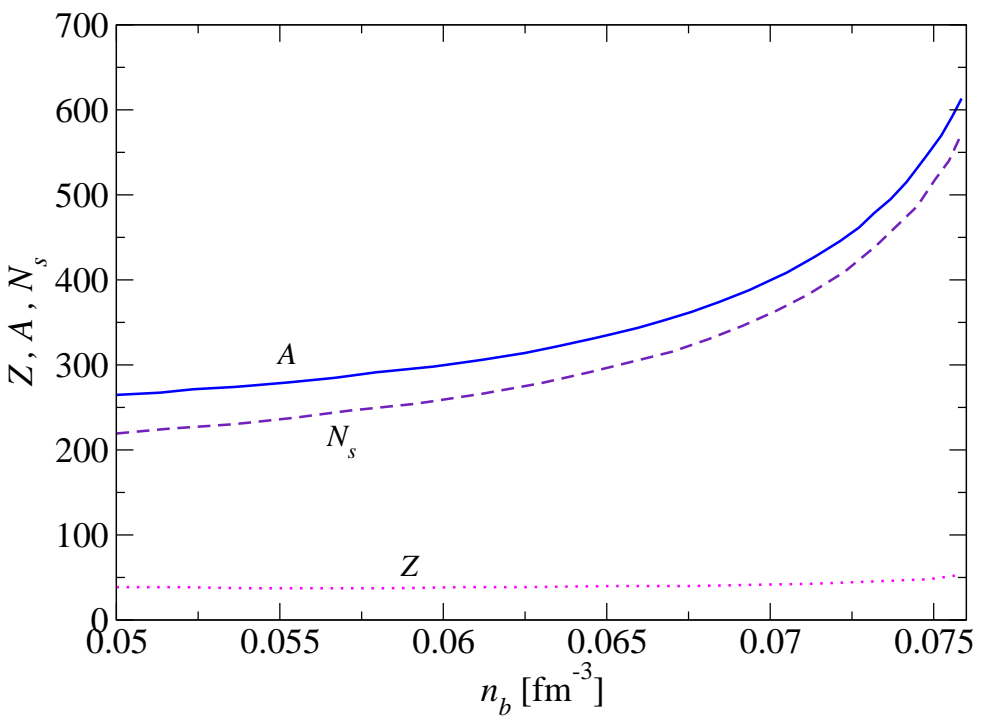

Figure 8: Composition of nuclear clusters in the ground state of the inner crust. Baryon number A of spherical clusters and their proton number Z, versus average baryon number density $n_{\mathrm{b}}$ as calculated by Douchin \& Haensel [124]. $N_{\mathrm{s}}$ is the number of neutrons adsorbed on the surface of the clusters. 


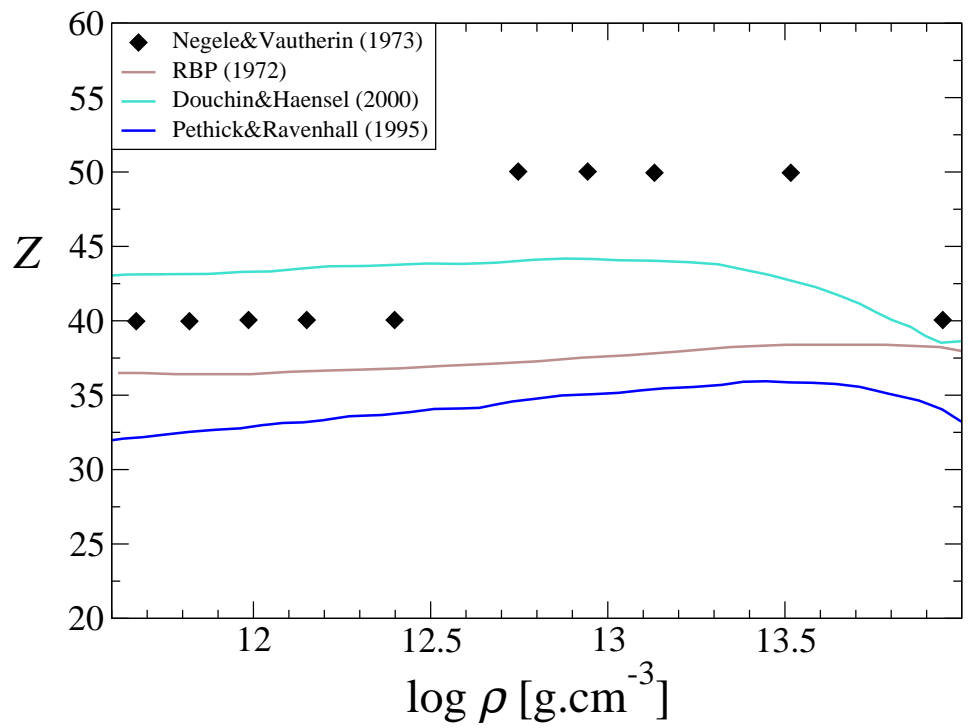

Figure 9: Proton number $Z$ of the nuclear clusters vs. density $\rho$ in the ground state of the inner crust of neutron stars, calculated by various authors from different liquid drop models based on many-body theories with effective interactions: RBP(Ravenhall, Bennett and Pethick) [344], Douchin \& Haensel [124], Pethick \& Ravenhall [326]. For comparison, the results of the quantum calculations of Negele \& Vautherin [303] (diamonds) are also shown.

various constituent particles. In this approach, the total energy density is written as a functional of the number densities of the different particle species

$$
\varepsilon(\boldsymbol{r})=\varepsilon_{\mathrm{N}}\left\{n_{n}(\boldsymbol{r}), n_{p}(\boldsymbol{r})\right\}+\varepsilon_{e}\left\{n_{e}(\boldsymbol{r})\right\}+\varepsilon_{\mathrm{Coul}}\left\{n_{e}(\boldsymbol{r}), n_{p}(\boldsymbol{r})\right\},
$$

where $\varepsilon_{\mathrm{N}}, \varepsilon_{e}$ and $\varepsilon_{\text {Coul }}$ are the nuclear, electron and Coulomb contributions, respectively.

The idea for obtaining the energy functional is to assume that the matter is locally homogeneous: this is known as the Thomas-Fermi or local density approximation. This approximation is valid when the characteristic length scales of the density variations are much larger than the corresponding interparticle spacings. The Thomas-Fermi approximation can be improved by including density gradients in the energy functional.

As discussed in Section 3.1, the electron density is almost constant so that the local density approximation is very good with the electron energy functional given by Equation (22).

The Coulomb part in Equation (52) can be decomposed into a classical and a quantum contribution. The classical contribution is given by

$$
\varepsilon_{\text {Coul }}^{\text {class }}\left\{n_{e}(\boldsymbol{r}), n_{p}(\boldsymbol{r})\right\}=\frac{1}{2} e\left(n_{p}(\boldsymbol{r})-n_{e}(\boldsymbol{r})\right) \phi(\boldsymbol{r}),
$$

where $e$ is the proton electric charge and $\phi$ is the electrostatic potential, which obeys Poisson's equation,

$$
\Delta \phi=-4 \pi e\left(n_{p}(\boldsymbol{r})-n_{e}(\boldsymbol{r})\right) .
$$

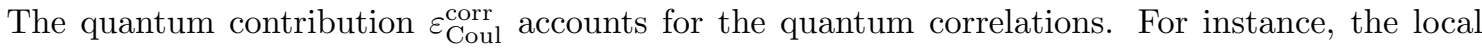
part of the Coulomb exchange correlations induced by the Pauli exclusion principle is given by the Slater-Kohn-Sham functional [243]

$$
\varepsilon_{\text {Coul }}^{\text {corr }}\left\{n_{e}(\boldsymbol{r}), n_{p}(\boldsymbol{r})\right\}=-\frac{3}{4}\left(\frac{3}{\pi}\right)^{1 / 3} e^{2}\left(n_{p}(\boldsymbol{r})^{4 / 3}+n_{e}(\boldsymbol{r})^{4 / 3}\right) .
$$


The nuclear functional $\varepsilon_{\mathrm{N}}\left\{n_{n}(\boldsymbol{r}), n_{p}(\boldsymbol{r})\right\}$ is less certain. Its local part is just a function of $n_{n}$ and $n_{p}$, and can, in principle, be inferred using the results of many-body calculations of the ground state of uniform asymmetric nuclear matter. However, the many-body calculations for the nonlocal part of the nuclear functional are much more difficult and have never been done in a fully satisfactory way. A simpler procedure is to postulate a purely phenomenological expression of the nonlocal part of the nuclear functional. The free parameters are then determined to reproduce some nuclear properties, for instance, the experimental atomic masses. Alternatively, the nuclear functional can be obtained from effective theories. In this case, the bare nucleon-nucleon interaction is replaced by an effective phenomenological interaction. It is then possible to deduce the nuclear functional in a systematic way using the extended Thomas-Fermi approximation (see for instance [58]). This approach has been developed for neutron star crust matter by Onsi and collaborators [312, 311].

The total energy density is equal to the energy density of one unit cell of the lattice times the number of cells. The minimization of the total energy density under the constraints of a fixed total baryon density $n_{\mathrm{b}}$ and global electro-neutrality

$$
n_{\mathrm{b}}=\frac{1}{\mathcal{V}_{\text {cell }}} \int_{\text {cell }} \mathrm{d}^{3} r\left(n_{n}(\boldsymbol{r})+n_{p}(\boldsymbol{r})\right), \quad \int_{\text {cell }} \mathrm{d}^{3} r\left(n_{p}(\boldsymbol{r})-n_{e}(\boldsymbol{r})\right)=0,
$$

leads to Euler-Lagrange equations for the nucleon densities. In practice the unit cell is usually approximated by a sphere of the same volume $\mathcal{V}_{\text {cell }}$. The boundary conditions are that the gradients of the densities and of the Coulomb potential vanish at the origin $r=0$ and on the surface of the sphere $r=R_{\text {cell }}$. Instead of solving the Euler-Lagrange equations, the nucleon densities are usually parameterized by some simple analytic functions with correct boundary behavior. Free parameters are then determined by minimizing the energy as in the compressible liquid drop models discussed in Section 3.2.1. The proton number of the nuclear clusters in the inner crust vs. mass density is shown in Figure 10 for different models. In those semiclassical models (as well as in liquid drop models discussed in Section 3.2.1), the number of bound nucleons inside the clusters in neutron star crusts varies continuously with depth. However, the nuclear clusters are expected to exhibit specific magic numbers of nucleons as similarly observed in isolated terrestrial nuclei, due to the clustering of quantum single-particle energy levels. The scattering of the unbound neutrons on the nuclear inhomogeneities leads also to "shell" (Casimir or band) effects [62, 278, 90]. The energy corrections due to shell effects have been studied perturbatively in semiclassical models [316, 130] and in Hartree-Fock calculations [278]. They have been found to be small. However, since the energy differences between different nuclear configurations are small, especially at high densities, these shell effects are important for determining the equilibrium structure of the crust. Calculations of the ground state structure of the crust, including proton shell effects, have recently been carried out by Onsi et al. [311]. As can be seen in Figure 11, these shell effects significantly change the composition of the clusters predicting proton magic numbers $Z=20,40,50$.

\subsubsection{Quantum calculations}

Quantum calculations of the structure of the inner crust were pioneered by Negele \& Vautherin [303]. These types of calculations have been improved only recently by Baldo and collaborators [29, 30]. Following the Wigner-Seitz approximation [422], the inner crust is decomposed into independent spheres, each of them centered at a nuclear cluster, whose radius is defined by Equation (16), as illustrated in Figure 5. The determination of the equilibrium structure of the crust thus reduces to calculating the composition of one of the spheres. Each sphere can be seen as an exotic "nucleus". The methods developed in nuclear physics for treating isolated nuclei can then be directly applied.

Starting from many-body calculations of uniform nuclear matter with realistic nucleon-nucleon interaction, and expanding the nucleon density matrix in relative and center of mass coordinates, Negele \& Vautherin [302] derived a set of nonlinear equations for the single particle wave functions

Living Reviews in Relativity

http://www. livingreviews . org//rr-2008-10 


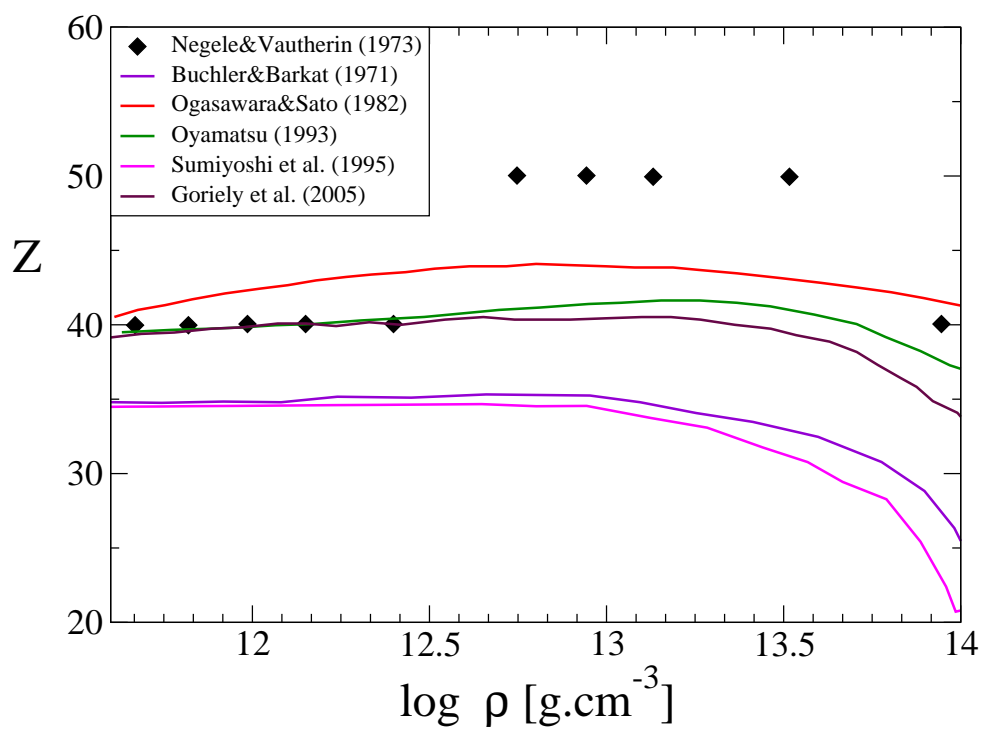

Figure 10: Proton number $Z$ of the nuclear clusters vs. the mass density $\rho$ in the ground state of the inner crust, calculated by different semi-classical models: Buchler \& Barkat [61], Ogasawara \& Sato [308], Oyamatsu [314], Sumiyoshi et al. [400], Goriely et al. [171]. For comparison the results of the quantum calculations of Negele \& Vautherin [303] (diamonds) are also shown.

of the nucleons, $\varphi_{\alpha}^{(q)}(\boldsymbol{r})$, where $q=n, p$ for neutron and proton, respectively, and $\alpha$ is the set of quantum numbers characterizing each single particle state. Inside the Wigner-Seitz sphere, these equations take the form

$$
-\boldsymbol{\nabla} \cdot \frac{\hbar^{2}}{2 m_{q}^{\oplus}(r)} \boldsymbol{\nabla} \varphi_{\alpha}^{(q)}(\boldsymbol{r})+U_{q}(r) \varphi_{\alpha}^{(q)}(\boldsymbol{r})+\frac{W_{q}(r)}{r} \boldsymbol{\ell} \cdot \boldsymbol{\sigma} \varphi_{\alpha}^{(q)}(\boldsymbol{r})=\epsilon_{\alpha}^{(q)} \varphi_{\alpha}^{(q)}(\boldsymbol{r}),
$$

where $\epsilon_{\alpha}^{(q)}$ is the single particle energy, $\boldsymbol{\ell} \equiv-\mathrm{i} \boldsymbol{r} \times \boldsymbol{\nabla}$ is the dimensionless orbital-angular-momentum operator and $\boldsymbol{\sigma}$ is a vector composed of Pauli spin matrices. The effective masses $m_{q}^{\oplus}(r)$, the mean fields $U_{q}(r)$ and the spin-orbit potentials $W_{q}(r)$ depend on wave functions of all nucleons inside the sphere through the particle number densities

$$
n_{q}(\boldsymbol{r})=\sum_{\alpha}\left|\varphi_{\alpha}^{(q)}(\boldsymbol{r})\right|^{2}
$$

the kinetic energy densities (in units of $\hbar^{2} / 2 m$, where $m$ is the nucleon mass)

$$
\tau_{q}(\boldsymbol{r})=\sum_{\alpha}\left|\nabla \varphi_{\alpha}^{(q)}(\boldsymbol{r})\right|^{2}
$$

and the spin-orbit densities

$$
\boldsymbol{J}_{\boldsymbol{q}}(\boldsymbol{r})=\boldsymbol{r} \sum_{\alpha} \varphi_{\alpha}^{(q)}(\boldsymbol{r})^{*} \frac{\boldsymbol{\ell} \cdot \boldsymbol{\sigma}}{r^{2}} \varphi_{\alpha}^{(q)}(\boldsymbol{r})
$$

Equations (57) reduce to ordinary differential equations by expanding a wave function on the basis of the total angular momentum. Apart from the nuclear central and spin-orbit potentials, the protons also feel a Coulomb potential. In the Hartree-Fock approximation, the Coulomb potential is the sum of a direct part $e \phi(\boldsymbol{r})$, where $\phi(\boldsymbol{r})$ is the electrostatic potential, which obeys 
Poisson's Equation (54), and an exchange part, which is nonlocal in general. Negele \& Vautherin adopted the Slater approximation for the Coulomb exchange, which leads to a local proton Coulomb potential. As a remark, the expression of the Coulomb exchange potential used nowadays was actually suggested by Kohn \& Sham [243]. It is smaller by a factor $3 / 2$ compared to that initially proposed by Slater [380] before the formulation of the density functional theory. It is obtained by taking the derivative of Equation (55) with respect to the proton density $n_{p}(r)$. Since the clusters in the crust are expected to have a very diffuse surface and a thick neutron skin (see Section 3.2.2), the spin-orbit coupling term for the neutrons (which is proportional to the gradient of the neutron density) was neglected.

Equations (57) have to be solved self-consistently. For a given number $N$ of neutrons and $Z$ of protons and some initial guess of the effective masses and potentials, the equations are solved for the wave functions of $N$ neutrons and $Z$ protons, which correspond to the lowest energies $\epsilon_{\alpha}^{(q)}$. These wave functions are then used to recalculate the effective masses and potentials. The process is iterated until the convergence is achieved.

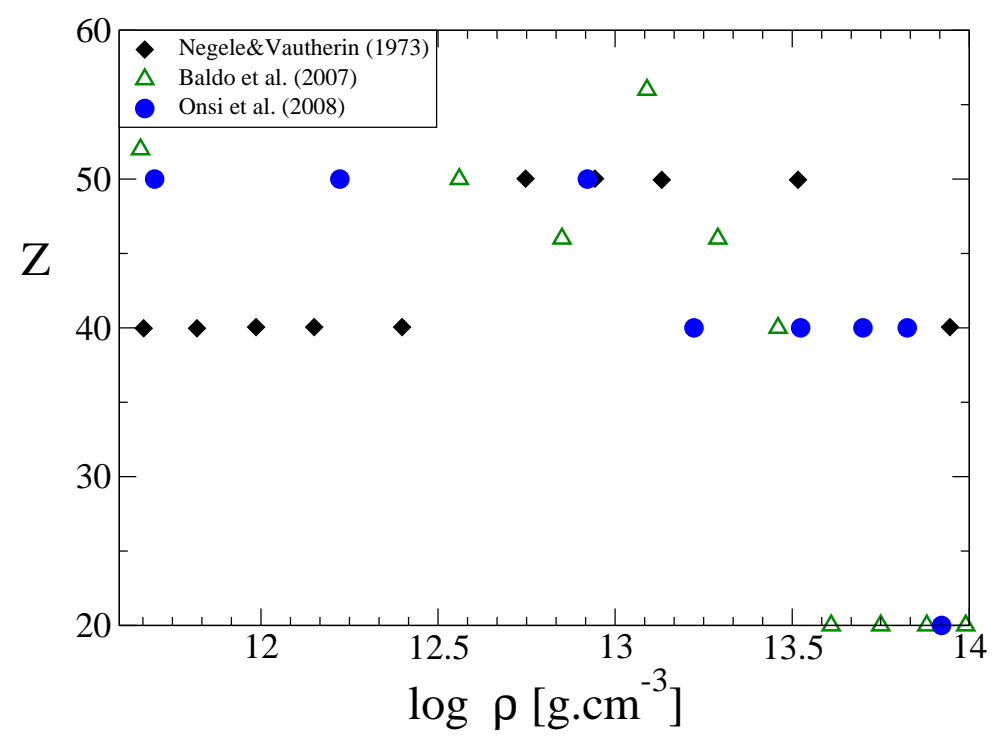

Figure 11: Proton number $Z$ of the nuclear clusters vs. the mass density $\rho$ in the ground state of the inner crust, calculated by different quantum models: Negele \& Vautherin [303], the model P2 from Baldo et al. [31, 32] and the recent calculations of Onsi et al [311].

Negele \& Vautherin [303] determined the structure of the inner crust by minimizing the total energy per nucleon in a Wigner-Seitz sphere, and thus treating the electrons as a relativistic Fermi gas. Since the sphere is electrically neutral, the number of electrons is equal to $Z$ and the electron energy is easily evaluated from Equation (22) with $n_{e}=Z / \mathcal{V}_{\text {cell }}$, where $\mathcal{V}_{\text {cell }}$ is the volume of the sphere. As for the choice of boundary conditions, Negele \& Vautherin imposed that wave functions with even parity (even $\ell$ ) and the radial derivatives of wave functions with odd parity (odd $\ell$ ) vanish on the sphere $r=R_{\text {cell }}$. This prescription leads to a roughly constant neutron density outside the nuclear clusters. However, the densities had still to be averaged in the vicinity of the cell edge in order to remove unphysical fluctuations. The structure and the composition of the inner crust is shown in Table 3. These results are qualitatively similar to those obtained with liquid drop models (see Figure 9 in Section 3.2.1) and semiclassical models (see Figure 10 in Section 3.2.2). The remarkable distinctive feature is the existence of strong proton quantum-shell effects with a predominance of nuclear clusters with $Z=40$ and $Z=50$. The same magic numbers have been

Living Reviews in Relativity

http://www. livingreviews.org/Irr-2008-10 
recently found by Onsi et al. [311] using a high-speed approximation to the Hartree-Fock method with an effective Skyrme force that was adjusted on essentially all nuclear data. Note however that the predicted sequence of magic numbers differs from that obtained by Negele and Vautherin as can be seen in Figure 11. Neutron quantum effects are also important (while not obvious from the table) as can be inferred from the spatial density fluctuations inside the clusters in Figure 12. This figure also shows that these quantum effects disappear at high densities, where the matter becomes nearly homogeneous. The quantum shell structure of nuclear clusters in neutron star crusts is very different from that of ordinary nuclei owing to a large number of neutrons (for a recent review on the shell structure of very neutron-rich nuclei, see, for instance, [119]). For instance, clusters with $Z=40$ are strongly favored in neutron star crusts, while $Z=40$ is not a magic number in ordinary nuclei (however, it corresponds to a filled proton subshell).

Table 3: Sequence of nuclear clusters in the ground state of the inner crust calculated by Negele \& Vautherin [303]. Here $N$ is the total number of neutrons in the Wigner-Seitz sphere (i.e., it is a sum of the number of neutrons bound in nuclei and of those forming a neutron gas, per nucleus). Isotopes are labelled with the total number of nucleons in the Wigner-Seitz sphere.

\begin{tabular}{ccccc}
\hline$\rho\left[\mathrm{g} \mathrm{cm}^{-3}\right]$ & Element & $Z$ & $N$ & $R_{\text {cell }}[\mathrm{fm}]$ \\
\hline $4.67 \times 10^{11}$ & ${ }^{180} \mathrm{Zr}$ & 40 & 140 & 53.60 \\
$6.69 \times 10^{11}$ & ${ }^{200} \mathrm{Zr}$ & 40 & 160 & 49.24 \\
$1.00 \times 10^{12}$ & ${ }^{250} \mathrm{Zr}$ & 40 & 210 & 46.33 \\
$1.47 \times 10^{12}$ & ${ }^{320} \mathrm{Zr}$ & 40 & 280 & 44.30 \\
$2.66 \times 10^{12}$ & ${ }^{500} \mathrm{Zr}$ & 40 & 460 & 42.16 \\
$6.24 \times 10^{12}$ & ${ }^{950} \mathrm{Sn}$ & 50 & 900 & 39.32 \\
$9.65 \times 10^{12}$ & ${ }^{1100} \mathrm{Sn}$ & 50 & 1050 & 35.70 \\
$1.49 \times 10^{13}$ & ${ }^{1350} \mathrm{Sn}$ & 50 & 1300 & 33.07 \\
$3.41 \times 10^{13}$ & ${ }^{1800} \mathrm{Sn}$ & 50 & 1750 & 27.61 \\
$7.94 \times 10^{13}$ & ${ }^{1500} \mathrm{Zr}$ & 40 & 1460 & 19.61 \\
$1.32 \times 10^{14}$ & ${ }^{982} \mathrm{Ge}$ & 32 & 950 & 14.38 \\
\hline
\end{tabular}

Negele \& Vautherin assume that nucleons can be described as independent particles in a mean field induced by all other particles. However, neutrons and protons are expected to form bound pairs due to the long-range attractive part of the nucleon-nucleon interaction, giving rise to the property of superfluidity (Section 8). Baldo and collaborators [28, 32, 29, 30, 31] have recently studied the effects of these pairing correlations on the structure of neutron star crusts, applying the generalized energy-density-functional theory in the Wigner-Seitz approximation. They found that the composition of the clusters differs significantly from that obtained by Negele \& Vautherin [303], as can be seen from Table 4 and Figure 11. However, Baldo et al. stressed that these results depend on the more-or-less arbitrary choice of boundary conditions imposed on the Wigner-Seitz sphere, especially in the deepest layers of the inner crust. Therefore, above $2 \times 10^{13} \mathrm{~g} \mathrm{~cm}^{-3}$ results of Baldo et al. (and those of Negele \& Vautherin) should be taken with a grain of salt. Another limitation of the Wigner-Seitz approximation is that it does not allow the calculation of transport properties, since neutrons are artificially confined inside the sphere. A more realistic treatment has been recently proposed by applying the band theory of solids (see [92] and references therein). 
Table 4: Sequence of nuclear clusters in the ground state of the inner crust calculated by Baldo et al. [31, 32] including pairing correlations (their P2 model). The boundary conditions are the same as those of Negele and Vautherin [303]. Similarly, $N$ is the total number of neutrons in the Wigner-Seitz sphere. The isotopes are labelled with the total number of nucleons in the Wigner-Seitz sphere, as in Table 3.

\begin{tabular}{ccccc}
\hline$\rho\left[\mathrm{g} \mathrm{cm}^{-3}\right]$ & Element & $Z$ & $N$ & $R_{\text {cell }}[\mathrm{fm}]$ \\
\hline $4.52 \times 10^{11}$ & ${ }^{212} \mathrm{Te}$ & 52 & 160 & 57.19 \\
$1.53 \times 10^{12}$ & ${ }^{562} \mathrm{Xe}$ & 54 & 508 & 52.79 \\
$3.62 \times 10^{12}$ & ${ }^{830} \mathrm{Sn}$ & 50 & 780 & 45.09 \\
$7.06 \times 10^{12}$ & ${ }^{1020} \mathrm{Pd}$ & 46 & 974 & 38.64 \\
$1.22 \times 10^{13}$ & ${ }^{1529} \mathrm{Ba}$ & 56 & 1473 & 36.85 \\
$1.94 \times 10^{13}$ & ${ }^{1351} \mathrm{Pd}$ & 46 & 1305 & 30.31 \\
$2.89 \times 10^{13}$ & ${ }^{1269} \mathrm{Zr}$ & 40 & 1229 & 25.97 \\
$4.12 \times 10^{13}$ & ${ }^{636} \mathrm{Cr}$ & 20 & 616 & 18.34 \\
$5.65 \times 10^{13}$ & ${ }^{642} \mathrm{Ca}$ & 20 & 622 & 16.56 \\
$7.52 \times 10^{13}$ & ${ }^{642} \mathrm{Ca}$ & 20 & 622 & 15.05 \\
$9.76 \times 10^{13}$ & ${ }^{633} \mathrm{Ca}$ & 20 & 613 & 13.73 \\
\hline
\end{tabular}
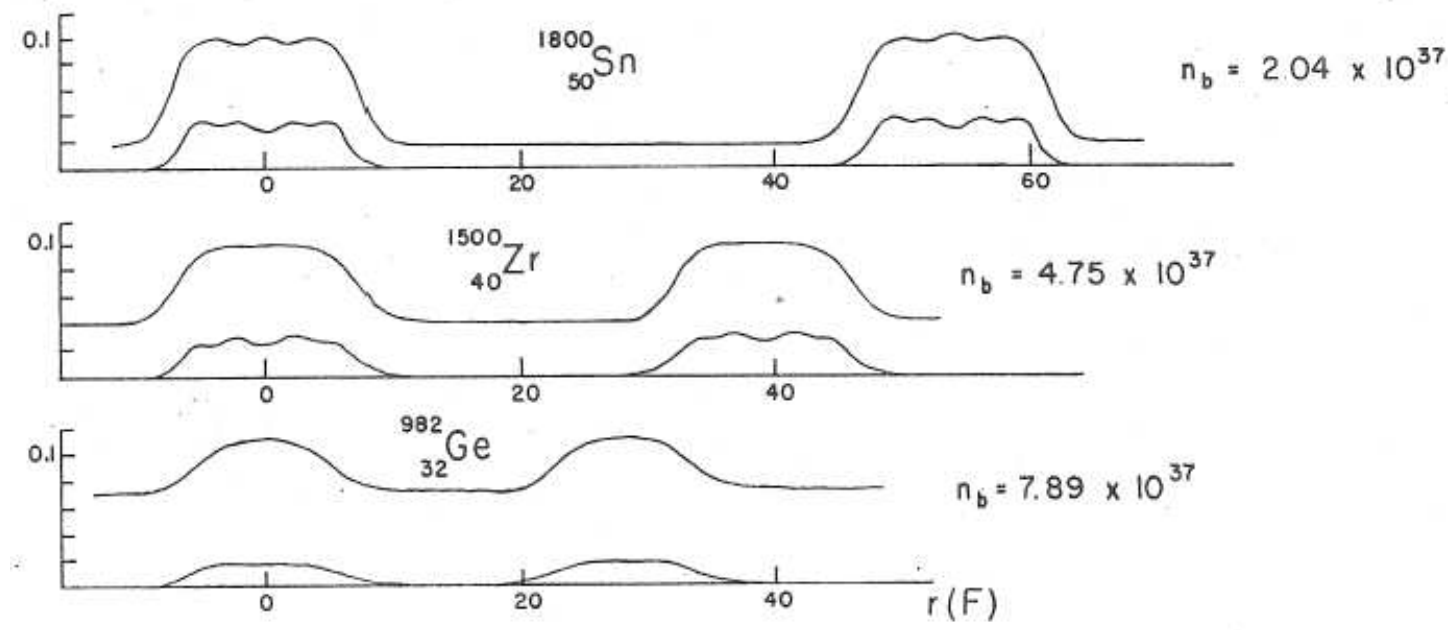

Figure 12: Nucleon number densities $\left(\mathrm{in}_{\mathrm{fm}}^{-3}\right.$ ) along the axis joining two adjacent Wigner-Seitz cells of the ground state of the inner crust, for a few baryon densities $n_{\mathrm{b}}\left(\right.$ in $\mathrm{cm}^{-3}$ ), as calculated by Negele \& Vautherin [303]. 


\subsubsection{Going further: nuclear band theory}

The unbound neutrons in the inner crust of a neutron star are closely analogous to the "free" electrons in an ordinary (i.e. under terrestrial conditions) metal ${ }^{2}$. Assuming that the ground state of cold dense matter below saturation density possesses the symmetry of a perfect crystal, which is usually taken for granted, it is therefore natural to apply the band theory of solids to neutron star crusts (see Carter, Chamel \& Haensel [78] for the application to the pasta phases and Chamel [90, 91] for the application to the general case of 3D crystal structures).

The band theory is explained in standard solid-state physics textbooks, for instance in the book by Kittel [241]. Single particle wave functions of nucleon species $q=n, p$ in the crust are characterized by a wave vector $k$ and obey the Floquet-Bloch theorem

$$
\varphi_{\boldsymbol{k}}^{(q)}(\boldsymbol{r}+\boldsymbol{T})=e^{\mathrm{i} \boldsymbol{k} \cdot \boldsymbol{T}} \varphi_{\boldsymbol{k}}^{(q)}(\boldsymbol{r})
$$

where $\boldsymbol{T}$ is any lattice translation vector (which transforms the lattice into itself). This theorem implies that the wave functions are modulated plane waves, called simply Bloch waves

$$
\varphi_{\boldsymbol{k}}^{(q)}(\boldsymbol{r})=e^{\mathrm{i} \boldsymbol{k} \cdot \boldsymbol{r}} u_{\boldsymbol{k}}^{(q)}(\boldsymbol{r})
$$

with $u_{\boldsymbol{k}}^{(q)}(\boldsymbol{r})$ having the full periodicity of the lattice, $u_{\boldsymbol{k}}^{(q)}(\boldsymbol{r}+\boldsymbol{T})=u_{\boldsymbol{k}}^{(q)}(\boldsymbol{r})$.

In the approach of Negele \& Vautherin [302] (see Section 3.2.3), or in the more popular mean field method with effective Skyrme nucleon-nucleon interactions [47, 391], single particle states are solutions of the equations

$$
-\nabla \cdot \frac{\hbar^{2}}{2 m_{q}^{\oplus}(\boldsymbol{r})} \nabla \varphi_{\boldsymbol{k}}^{(q)}(\boldsymbol{r})+U_{q}(\boldsymbol{r}) \varphi_{\boldsymbol{k}}^{(q)}(\boldsymbol{r})-\mathrm{i} \boldsymbol{W}_{\boldsymbol{q}}(\boldsymbol{r}) \cdot \nabla \times \boldsymbol{\sigma} \varphi_{\boldsymbol{k}}^{(q)}(\boldsymbol{r})=\epsilon^{(q)}(\boldsymbol{k}) \varphi_{\boldsymbol{k}}^{(q)}(\boldsymbol{r}),
$$

neglecting pairing correlations (the application of band theory including pairing correlations has been discussed in [77]). Despite their apparent simplicity, these equations are highly nonlinear, since the various quantities depend on the wave functions (see Section 3.2.3).

As a result of the lattice symmetry, the crystal can be partitioned into identical primitive cells, each containing exactly one lattice site. The specification of the primitive cell is not unique. A particularly useful choice is the Wigner-Seitz or Voronoi cell, defined by the set of points that are closer to a given lattice site than to any other. This cell is very convenient since it reflects the local symmetry of the crystal. The Wigner-Seitz cell of a crystal lattice is a complicated polyhedron in general. For instance, the Wigner-Seitz cell of a body-centered cubic lattice (which is the expected ground state structure of neutron star crusts), shown in Figure 13, is a truncated octahedron.

Equations (63) need to be solved inside only one such cell. Indeed once the wave function in one cell is known, the wave function in any other cell can be deduced from the Floquet-Bloch theorem (61). This theorem also determines the boundary conditions to be imposed at the cell boundary.

For each wave vector $\boldsymbol{k}$, there exists only a discrete set of single particle energies $\epsilon_{\alpha}^{(q)}(\boldsymbol{k})$, labeled by the principal quantum number $\alpha$, for which the boundary conditions (61) are fulfilled. The energy spectrum is thus formed of "bands", each of them being a continuous (but in general not analytic) function of the wave vector $\boldsymbol{k}$ (bands are labelled by increasing values of energy, so that $\epsilon_{\alpha}^{(q)}(\boldsymbol{k}) \leq \epsilon_{\beta}^{(q)}(\boldsymbol{k})$ if $\left.\alpha<\beta\right)$. The band index $\alpha$ is associated with the rotational symmetry of the nuclear clusters around each lattice site, while the wave vector $k$ accounts for the translational symmetry. Both local and global symmetries are therefore properly taken into account. Let us remark that the band theory includes uniform matter as a limiting case of an "empty" crystal.

\footnotetext{
2 "free" means here that the electrons are not bound. However, they are interacting with other electrons and with the atomic lattice.
} 


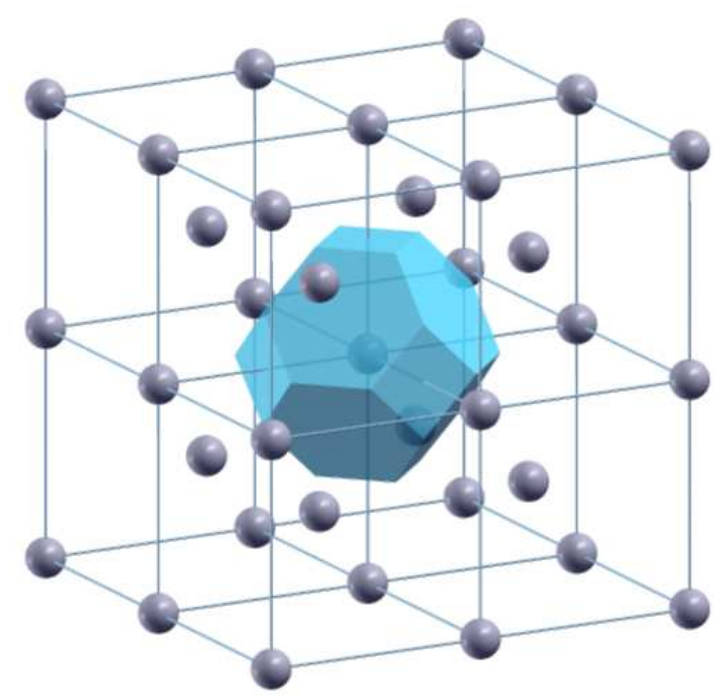

Figure 13: Wigner-Seitz cell of a body-centered cubic lattice.

In principle, Equations (63) have to be solved for all wave vectors $\boldsymbol{k}$. Nevertheless, it can be shown by symmetry that the single particle states (and, therefore, the single particle energies) are periodic in $\boldsymbol{k}$-space

$$
\varphi_{\boldsymbol{k}+\boldsymbol{G}}^{(q)}(\boldsymbol{r})=\varphi_{\boldsymbol{k}}^{(q)}(\boldsymbol{r})
$$

where the reciprocal lattice vectors $\boldsymbol{G}$ are defined by

$$
G \cdot T=2 \pi N
$$

$N$ being any positive or negative integer. The discrete set of all possible reciprocal vectors $\boldsymbol{G}$ defines a reciprocal lattice in $\boldsymbol{k}$-space. Equation (64) entails that only the wave vectors $\boldsymbol{k}$ lying inside the first Brillouin zone (i.e. Wigner-Seitz cell of the reciprocal lattice) are relevant. The first Brillouin zone of a body-centered cubic lattice is shown in Figure 14.

An example of neutron band structure is shown in the right panel of Figure 15 from Chamel et al.[96]. The figure also shows the energy spectrum obtained by removing the nuclear clusters (empty lattice), considering a uniform gas of unbound neutrons. For comparison, the single particle energies, given in this limiting case by an expression of the form $\epsilon(\boldsymbol{k})=\hbar^{2} k^{2} /\left(2 m_{n}^{\oplus}\right)+U_{n}$, have been folded into the first Brillouin zone (reduced zone scheme). It can, thus, be seen that the presence of the nuclear clusters leads to distortions of the parabolic energy spectrum, especially at wave vectors $\boldsymbol{k}$ lying on Bragg planes (i.e., Brillouin zone faces, see Figure 14).

The (nonlinear) three-dimensional partial differential Equations (63) are numerically very difficult to solve (see Chamel [90, 91] for a review of some numerical methods that are applicable to neutron star crusts). Since the work of Negele \& Vautherin [303], the usual approach has been to apply the Wigner-Seitz approximation [422]. The complicated Wigner-Seitz cell (shown in Figure 13) is replaced by a sphere of equal volume. It is also assumed that the clusters are spherical so 


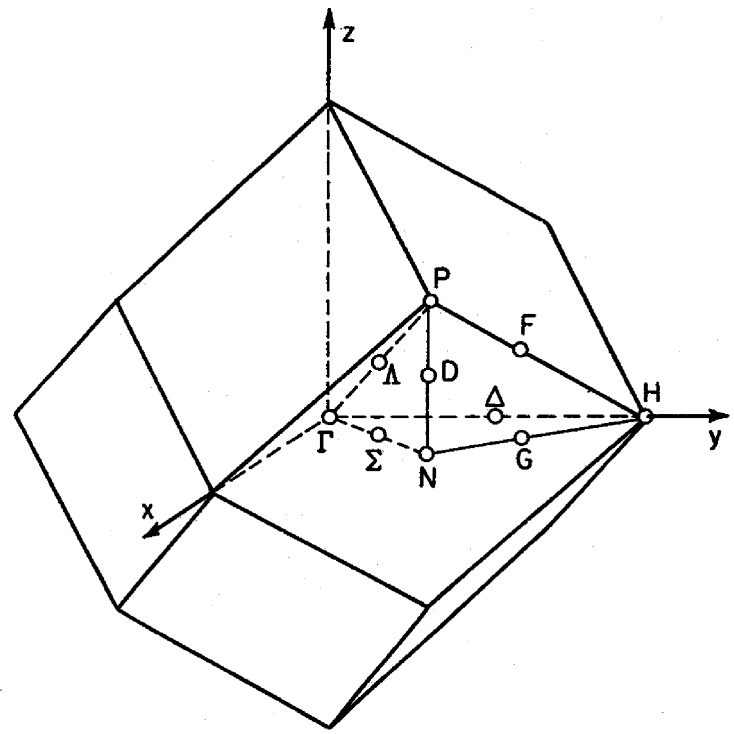

Figure 14: First Brillouin zone of the body-centered cubic lattice (whose Wigner-Seitz is shown in Figure 13). The directions $x, y$ and $z$ denote the Cartesian axis in $\boldsymbol{k}$-space.

that Equations (63) reduce to ordinary differential Equations (57). The Wigner-Seitz approximation has been used to predict the structure of the crust, the pairing properties, the thermal effects, and the low-lying energy-excitation spectrum of the clusters [303, 55, 360, 237, 413, 31, 294].

However, the Wigner-Seitz approximation overestimates the importance of neutron shell effects, as can be clearly seen in Figure 15. The energy spectrum is discrete in the Wigner-Seitz approximation (due to the neglect of the $\boldsymbol{k}$-dependence of the states), while it is continuous in the full band theory. The spurious shell effects depend on a particular choice of boundary conditions, which are not unique. Indeed as pointed out by Bonche \& Vautherin [54], two types of boundary conditions are physically plausible yielding a more-or-less constant neutron density outside the cluster: either the wave function or its radial derivative vanishes at the cell edge, depending on its parity. Less physical boundary conditions have also been applied, like the vanishing of the wave functions. Whichever boundary conditions are adopted, they lead to unphysical spatial fluctuations of the neutron density, as discussed in detail by Chamel et al. [96]. Negele \& Vautherin [303] average the neutron density in the vicinity of the cell edge in order to remove these fluctuations, but it is not clear whether this ad hoc procedure did remove all the spurious contributions to the total energy. As shown in Figure 15, shell energy gaps are on the order of $\Delta \epsilon \sim 100 \mathrm{keV}$, at $\rho \simeq 7 \times 10^{11} \mathrm{~g} \mathrm{~cm}^{-3}$. Since these gaps scale approximately like $\Delta \epsilon \propto \hbar^{2} /\left(2 m_{n} R_{\text {cell }}^{2}\right)$ (where $m_{n}$ is the neutron mass), they increase with density $\rho$ and eventually become comparable to the total energy difference between neighboring configurations. As a consequence, the predicted equilibrium structure of the crust becomes very sensitive to the choice of boundary conditions in the bottom layers $[30,96]$. One way of eliminating the boundary condition problem without carrying out full band structure calculations, is to perform semi-classical calculations including only proton shell effects with the Strutinsky method, as discussed by Onsi et al.[311].

In recent calculations $[278,304,168]$ the Wigner-Seitz cell has been replaced by a cube with periodic boundary conditions instead of Bloch boundary conditions (61). Although such calculations allow for possible deformations of the nuclear clusters, the lattice periodicity is still not properly taken into account, since such boundary conditions are associated with only one kind of solutions with $\boldsymbol{k}=0$. Besides the Wigner-Seitz cell is only cubic for a simple cubic lattice and it is 

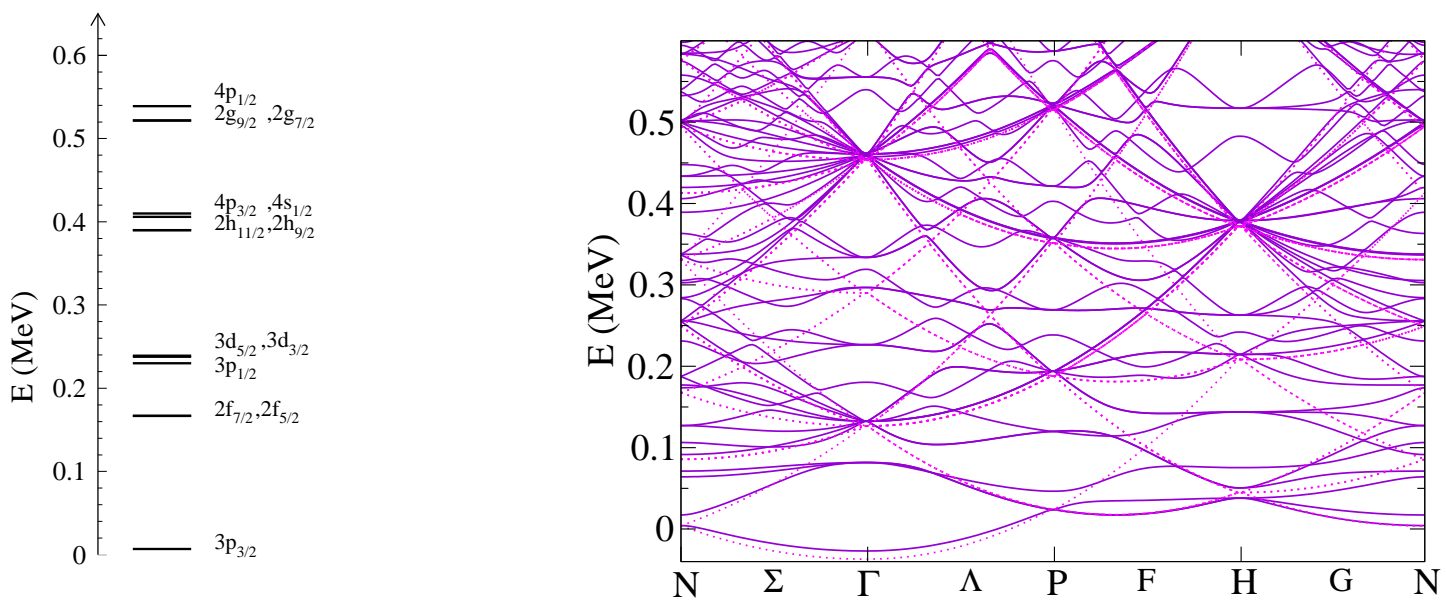

Figure 15: Single particle energy spectrum of unbound neutrons in the ground state of the inner crust composed of ${ }^{200} \mathrm{Zr}$, at $\rho \simeq 7 \times 10^{11} \mathrm{~g} \mathrm{~cm}^{-3}$, obtained by Chamel et al. [96]. Left: calculation in the Wigner-Seitz approximation. Right: full band structure calculation (reduced zone scheme) assuming that the crust is a body-centered cubic lattice with nuclear clusters (solid line) and without (dashed line). The capital letters on the horizontal axis refer to lines or points in $\mathbf{k}$-space, as indicated in Figure 14.

very unlikely that the equilibrium structure of the crust is of this type (the structure of the crust is expected to be a body centered cubic lattice as discussed in Section 3.1). Let us also remember that a simple cubic lattice is unstable. It is, therefore, not clear whether these calculations, which require much more computational time than those carried out in the spherical approximation, are more realistic. This point should be clarified in future work by a detailed comparison with full band theory. Let us also mention that recently Bürvenich et al. [64] have considered axially-deformed spheroidal W-S cells to account for deformations of the nuclear clusters.

Whereas the Wigner-Seitz approximation is reasonable at not too high densities for determining the equilibrium crust structure, full band theory is indispensable for studying transport properties (which involve obviously translational symmetry and, hence, the $\boldsymbol{k}$-dependence of the states). Carter, Chamel \& Haensel [78] using this novel approach have shown that the unbound neutrons move in the crust as if they had an effective mass much larger than the bare mass (see Sections 8.3.6 and 8.3.7). This dynamic effective neutron mass has been calculated by Carter, Chamel \& Haensel [78] in the pasta phases of rod and slab-like clusters (discussed in Section 3.3) and by Chamel $[90,91]$ in the general case of spherical clusters. By taking consistently into account both nuclear clusters, which form a solid lattice, and the neutron liquid, band theory provides a unified scheme for studying the structure and properties of neutron star crusts.

\subsection{Pastas}

The equilibrium structure of nuclear clusters in neutron star crusts results from the interplay between the total Coulomb energy and the surface energy of the nuclei according to the virial Equation (49). At low densities, the lattice energy Equation (41) is a small contribution to the total Coulomb energy and nuclear clusters are therefore spherical. However, at the bottom of the crust the nuclei are very close to one another. Consequently, the lattice energy represents a large reduction of the total Coulomb energy, which vanishes in the liquid core when the nuclear clusters fill all space, as can be seen from Equation (51). In the densest layers of the crust the Coulomb energy is comparable in magnitude to the net nuclear binding energy (this situation also occurs in the dense and hot collapsing core of a supernova and in heavy ion collisions leading to multifrag-

Living Reviews in Relativity

http://www. livingreviews.org/Irr-2008-10 
mentation [56, 57]). The matter thus becomes frustrated and can arrange itself into various exotic configurations as observed in complex fluids. For instance, surfactants are organic compounds composed of a hydrophobic "tail" and a hydrophilic "head". In solutions, surfactants aggregate into ordered structures, such as spherical or tubular micelles or lamellar sheets. The transition between the different phases is governed by the ratio between the volume of the hydrophobic and hydrophilic parts as shown in Figure 16. One may expect by analogy that similar structures could occur in the inner crust of a neutron star depending on the nuclear packing.

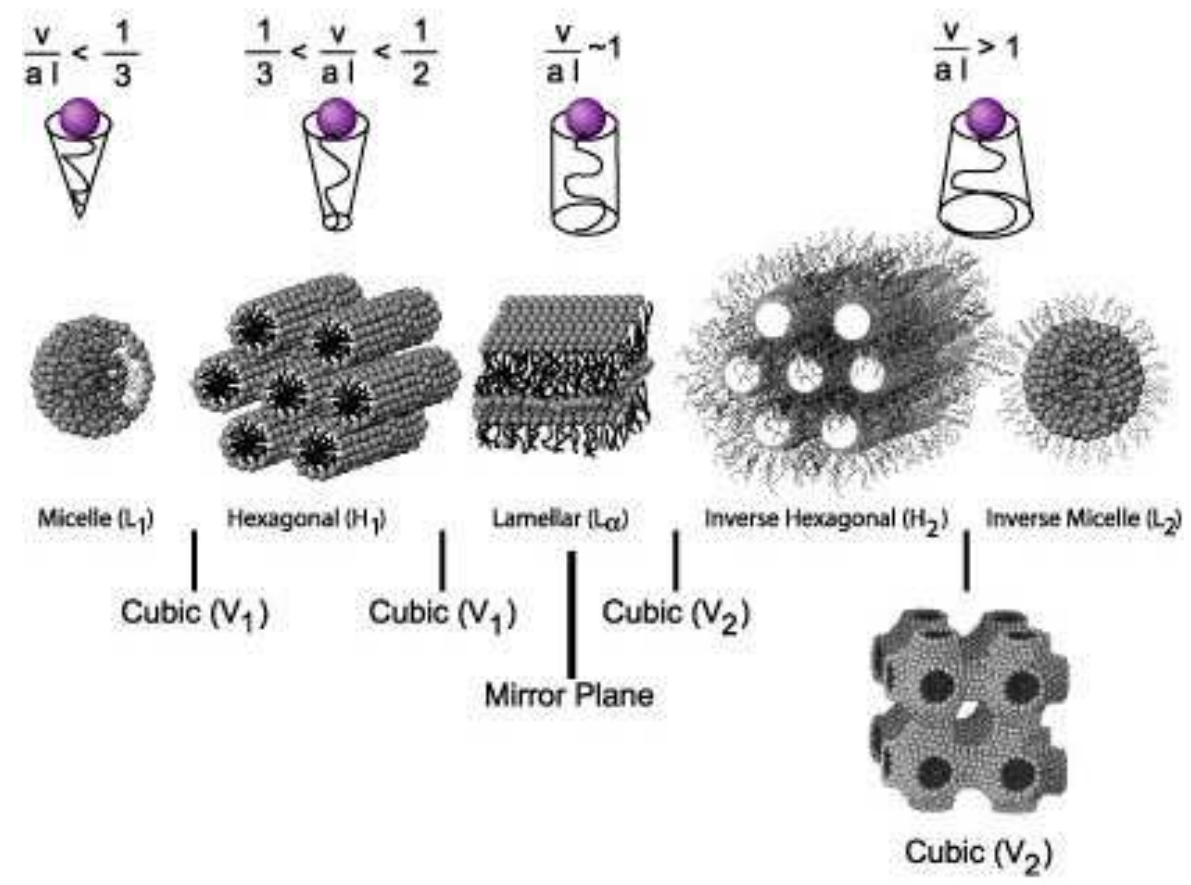

Figure 16: Structures formed by self-assembled surfactants in aqueous solutions, depending on the volume ratio of the hydrophilic and hydrophobic parts. Adapted from [226].

According to the Bohr-Wheeler fission condition [52] an isolated spherical nucleus in vacuum is stable with respect to quadrupolar deformations if

$$
E_{\mathrm{N}, \text { Coul }}^{(0)}<2 E_{\mathrm{N}, \text { surf }}^{(0)}
$$

where $E_{\mathrm{N} \text {,Coul }}^{(0)}$ and $E_{\mathrm{N} \text {,surf }}^{(0)}$ are the Coulomb and surface energies of the nucleus, respectively. The superscript (0) reminds us that we are considering a nucleus in vacuum. The Bohr-Wheeler condition can be reformulated in order to be applied in the inner crust, where both Coulomb and surface energies are modified compared to the "in vacuum" values. Neglecting curvature corrections and expanding all quantities to the linear order in $w^{1 / 3}$, where $w$ is the fraction of volume occupied by the clusters, it is found [326] that spherical clusters become unstable to quadrupolar deformation if $w>w_{\text {crit }}=1 / 8$.

Reasoning by analogy with percolating networks, Ogasawara \& Sato [308] suggest that as the nuclei fill more and more space, they will eventually deform, touch and merge to form new structures. A long time ago, Baym, Bethe and Pethick [39] predicted that as the volume fraction exceeds $1 / 2$, the crust will be formed of neutron bubbles in nuclear matter. In the general framework of the compressible liquid drop model considering the simplest geometries, Hashimoto and 
his collaborators $[191,315]$ show that as the nuclear volume fraction $w$ increases, the stable nuclear shape changes from sphere to cylinder, slab, tube and bubble, as illustrated in Figure 17. This sequence of nuclear shapes referred to as "pastas" (the cylinder and slab shaped nuclei resembling "spaghetti" and "lasagna" respectively) was found independently by Ravenhall et al. [345] with a specific liquid drop model. The volume fractions at which the various phases occur are in good agreement with those predicted by Hashimoto and collaborators on purely geometrical grounds. This criterion, however, relies on a liquid drop model, for which curvature corrections to the surface energy are neglected. This explains why some authors [274, 124] find within the liquid drop model that spherical nuclei remain stable down to the transition to uniform nuclear matter, despite volume fractions exceeding the critical threshold (see in particular Figure 7), while other groups found the predicted sequence of pasta phases [419, 420, 206, 207]. The nuclear curvature energy is, thus, important for predicting the equilibrium shape of the nuclei at a given density [327].

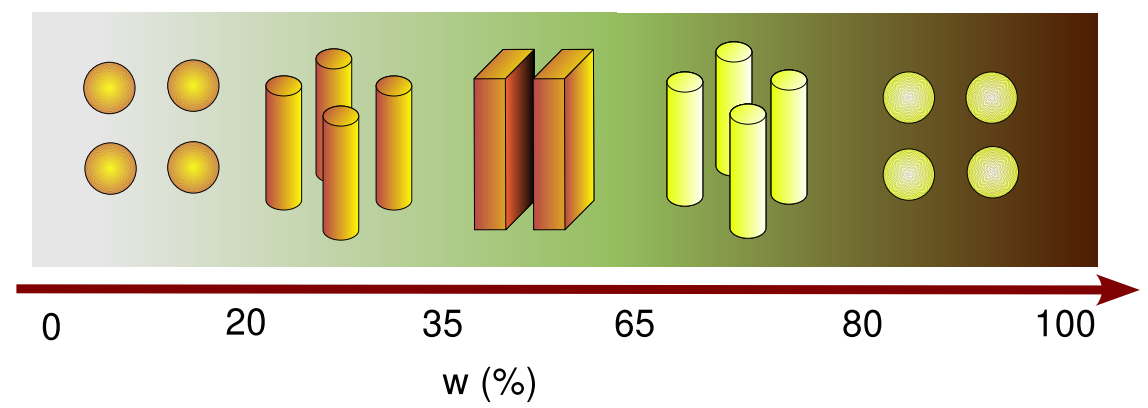

Figure 17: Sketch of the sequence of pasta phases in the bottom layers of ground-state crusts with an increasing nuclear volume fraction, based on the study of Oyamatsu and collaborators [315].

These pasta phases have been studied by various nuclear models, from liquid drop models to semiclassical models, quantum molecular dynamic simulations and Hartree-Fock calculations (for the current status of this issue, see, for instance, [417]). These models differ in numerical values of the densities at which the various phases occur, but they all predict the same sequence of configurations shown in Figure 17 (see also the discussion in Section 5.4 of [326]). Some models [274, 97, 124, 282] predict that the spherical clusters remain energetically favored throughout the whole inner crust. Generalizing the Bohr-Wheeler condition to nonspherical nuclei, Iida et al. [206, 207] showed that the rod-like and slab-like clusters are stable against fission and proton clustering, suggesting that the crust layers containing pasta phases may be larger than that predicted by the equilibrium conditions. It has also been suggested that the pinning of neutron superfluid vortices in neutron star crusts might trigger the formation of rod-like clusters [293]. Nevertheless, the nuclear pastas may be destroyed by thermal fluctuations [419, 420]. Quite remarkably, Watanabe and collaborators [417] performed quantum molecular dynamic simulations and observed the formation of rod-like and slab-like nuclei by cooling down hot uniform nuclear matter without any assumption of the nuclear shape. They also found the appearance of intermediate sponge-like structures, which might be identified with the ordered, bicontinuous, double-diamond geometry observed in block copolymers [284]. Those various phase transitions leading to the pasta structures in neutron star crusts are also relevant at higher densities in neutron star cores, where kaonic or quark pastas could exist [281].

The pasta phases cover a small range of densities near the crust-core interface with $\rho \sim$ $10^{14} \mathrm{~g} \mathrm{~cm}^{-3}$. Nevertheless, by filling the densest layers of the crust, they may represent a sizable fraction of the crustal mass [274] and thus may have important astrophysical consequences. For instance, the existence of nuclear pastas in hot dense matter below saturation density affects the neutrino opacity [201, 384], which is an important ingredient for understanding the gravita-

Living Reviews in Relativity

http://www . livingreviews . org//rr-2008-10 
tional core collapse of massive stars in supernova events and the formation of neutron stars (see Section 12.1). The dynamics of neutron superfluid vortices, which is thought to underlie pulsar glitches (see Section 12.4), is likely to be affected by the pasta phase. Besides, the presence of nonspherical clusters in the bottom layers of the crust influences the subsequent cooling of the star, hence the thermal X-ray emission by allowing direct Urca processes [274, 179] (see Section 11) and enhancing the heat capacity $[112,113,135]$. The elastic properties of the nuclear pastas can be calculated using the theory of liquid crystals [325, 419, 420] (see Section 7.2). The pasta phase could thus affect the elastic deformations of neutron stars, oscillations, precession and crustquakes.

\subsection{Impurities and defects}

There are many reasons why the real crust of neutron stars can be imperfect. In particular, apart from a dominating $(A, Z)$ nuclide at a given density $\rho$, it can contain an admixture of different nuclei ("impurities"). The initial temperature at birth exceeds $10^{10} \mathrm{~K}$. At such a high $T$, thermodynamic equilibrium is characterized by a statistical distribution of $A$ and $Z$. With decreasing $T$, the $A, Z$ peak becomes narrower $[55,63]$. After crystallization at $T_{\mathrm{m}}$, the composition is basically frozen. Therefore, the composition at $T<T_{\mathrm{m}}$ reflects the situation at $T \sim T_{\mathrm{m}}$, which can differ from that in the absolute ground state at $T=0$. For example, between the neighboring shells, with nuclides $\left(A_{1}, Z_{1}\right)$ and $\left(A_{2}, Z_{2}\right)$, respectively, one might expect a transition layer composed of a binary mixture of the two nuclides $[108,109]$. Another way of forming impurities is via thermal fluctuations of $Z$ and $N_{\text {cell }}$, which, according to Jones [224, 225], might be quite significant at $\rho \gtrsim 10^{12} \mathrm{~g} \mathrm{~cm}^{-3}$ and $T \gtrsim 10^{9} \mathrm{~K}$.

The real composition of neutron star crusts can also differ from the ground state due to the fallback of material from the envelope ejected during the supernova explosion and due to the accretion of matter. In particular, an accreted crust is a site of X-ray bursts. The ashes of unstable thermonuclear burning at accretion rates $10^{-8} M_{\odot} \mathrm{y}^{-1} \gtrsim \dot{M} \gtrsim 10^{-9} M_{\odot} \mathrm{y}^{-1}$ could be a mixture of $A \simeq 60-100$ nuclei and could therefore be relatively "impure" (heterogeneous and possibly amorphous) [365, 364]. If the initial ashes are a mixture of many nuclides, further compression under the weight of accreted matter can keep the heterogeneity. If the crust is weakly impure but rather amorphous, its thermal and electrical conductivities in the solid phase would be orders of magnitude lower than in the perfect crystal as discussed in Section 9. This would have dramatic consequences as far as the rate of the thermal relaxation of the crust is concerned (Section 12.7.3). 


\section{Accreting Neutron Star Crusts}

\subsection{Accreting neutron stars in low-mass X-ray binaries}

In this section we will concentrate on accreting neutron stars in low-mass X-ray binaries (LMXB), where the mass of the companion is significantly less than $M_{\odot}$, and the accretion stage can last as long as $\sim 10^{9} \mathrm{y}$. A binary is sufficiently tight for the companion to fill its Roche lobe. The mass transfer proceeds through the inner Lagrangian point, and the transferred plasma flows in a deep gravitational potential well, via an accretion disk, towards the neutron star surface, as illustrated in Figure 18.

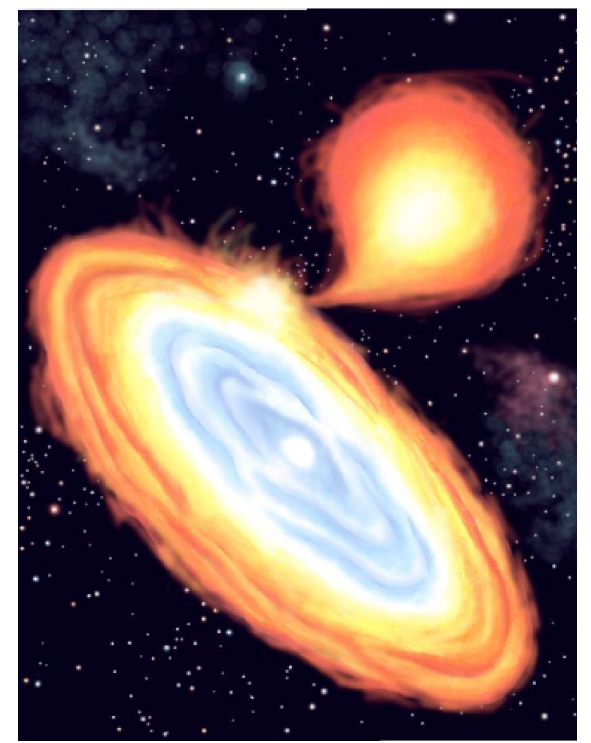

Figure 18: The artist's view of a low-mass X-ray binary. The companion of a neutron star fills its Roche lobe and loses its mass via plasma flow through the inner Lagrangian point. Due to its angular momentum, plasma orbits around the neutron star, forming an accretion disk. Gradually losing angular momentum due to viscosity within the accretion disk, plasma approaches the neutron star and eventually falls onto its surface. Figure by T. Piro.

A hydrogen atom falling on a neutron star surface from infinity releases $\sim 200 \mathrm{MeV}$ of gravitational binding energy. Therefore, accretion onto a neutron star releases $\sim 200 \mathrm{MeV}$ per accreted nucleon. Most of this energy is radiated in X-rays, so that the total X-ray luminosity of an accreting neutron star can be estimated as $L_{\mathrm{X}} \sim\left(\dot{M} / 10^{-10} M_{\odot} / \mathrm{y}\right) 10^{36} \mathrm{erg} \mathrm{s}^{-1}$. Space X-ray observations of accreting neutron stars were at the origin of X-ray astronomy [159]. Accreted matter is usually hydrogen rich. It forms the outer envelope of a neutron star, which contains a hydrogen burning shell, with an energy release of about $5 \mathrm{MeV} /$ nucleon in a stable burning. Helium ashes from hydrogen burning accumulate in the helium layer, which ignites under specific density-temperature conditions. For some range of accretion rate, helium burning is unstable, so that its ignition triggers a thermonuclear flash, burning within seconds all the envelope into nuclear ashes composed of nuclides of the iron group and beyond it; the energy release in the flash is less than $5 \mathrm{MeV} /$ nucleon. These flashes are observed as X-ray bursts, with luminosity rising in a second to about $10^{38} \mathrm{ergs}^{-1}$ $\left(\approx\right.$ Eddington limit for neutron stars, $\left.L_{\mathrm{Edd}}\right)$, and then typically decaying in a few tens of seconds ${ }^{3}$.

\footnotetext{
${ }^{3}$ We restrict ourselves to type I X-ray bursts. There are two X-ray bursters that are of type II, with bursts driven not by thermonuclear flashes on the neutron star surface, but originating in the accretion disk itself.
} 
Multiplying the burst luminosity by its duration we get an estimate of the total burst energy $\sim 10^{39}-10^{40}$ erg. The X-ray bursts are quasiperiodic, with typical recurrence time $\sim$ hoursdays. Since their discovery in 1975 [177], about seventy X-ray bursters have been found. Many bursters are of transient character, and form a group of soft X-ray transients (SXTs), with typical active periods of days - weeks, separated by periods of quiescence of several months - years long. During quiescent periods, there is very little or no accretion, while during much shorter periods of activity there is an abundant accretion, due probably to disc flow instability. Some SXTs, with active periods of years separated by decades of quiescence, are called persistent SXTs. In 2000, a special rare type of X-ray superbursts was discovered. Superbursts last for a few to twelve hours, with recurrence times of several years. The total energy radiated in a superburst is $\sim 10^{42} \mathrm{erg}$. Superbursts are explained by the unstable burning of carbon in deep layers of the outer crust.

In all cases, ignition of the thermonuclear flash takes place in the neutron star crust, and is sensitive to the crust structure and to the physical conditions within it. This aspect will be discussed in Section 4.4. An accreted crust has a different structure and composition than the ground state one, as discussed in Section 4.2. It has, therefore, a different equation of state than the ground-state crust (see Section 5.2). Moreover, it is a reservoir of nuclear energy, which is released in the process of deep crustal heating, accompanying accretion, reviewed in Section 4.3. Observations of SXTs in quiescence prove the presence of deep crustal heating (Section 12.7.2). Cooling of the neutron star surface in quiescence after long periods of accretion (years - decades) in persistent SXTs also allows one to test physical properties of the accreted crust (Section 12.7.3).

\subsection{Nuclear processes and formation of accreted crusts}

In this section we will describe the fate of X-ray burst ashes, produced at $\lesssim 10^{7} \mathrm{~g} \mathrm{~cm}^{-3}$, and then sinking deeper and deeper under the weight of accreted plasma above them. We start at a few tens of meters below the surface, and we will end at a depth of $\sim 1 \mathrm{~km}$, where the density $\gtrsim 10^{13} \mathrm{~g} \mathrm{~cm}^{-3}$. Under conditions prevailing in accreting neutron star crusts, at $\rho>10^{8} \mathrm{~g} \mathrm{~cm}^{-3}$ matter is strongly degenerate, and is "relatively cold" $\left(T \lesssim 10^{8} \mathrm{~K}\right.$, see Figures 25 and 26$)$, so that thermonuclear processes are strongly suppressed because interacting nuclei have to overcome a large Coulomb barrier. The structure of an accreted crust is shown in Figure 19.

In what follows we will use a simple model of the accreted crust formation, based on the onecomponent plasma approximation at $T=0[185,187]$. The (initial) X-burst ashes are approximated by a one-component plasma with $\left(A_{\mathrm{i}}, Z_{\mathrm{i}}\right)$ nuclei.

At densities lower than the threshold for pycnonuclear fusion (which is very uncertain, see Yakovlev et al. [427]), $\rho_{\text {pyc }} \sim 10^{12}-10^{13} \mathrm{~g} \mathrm{~cm}^{-3}$, the number of nuclei in an element of matter does not change during the compression resulting from the increasing weight of accreted matter. Due to nucleon pairing, stable nuclei in dense matter have even $N=A-Z$ and $Z$ (even-even nuclides). In the outer crust, in which free neutrons are absent, the electron captures proceed in two steps,

$$
\begin{gathered}
(A, Z)+e^{-} \longrightarrow(A, Z-1)+\nu_{e}, \\
(A, Z-1)+e^{-} \longrightarrow(A, Z-2)+\nu_{e}+Q_{j} .
\end{gathered}
$$

Electron captures lead to a systematic decrease in $Z$ (therefore an increase in $N=A-Z$ ) with increasing density. The first capture, Equation (67), proceeds as soon as $\mu_{e}>E\{A, Z-1\}-$ $E\{A, Z\}$, in a quasi-equilibrium manner, with negligible energy release. It produces an oddodd nucleus, which is strongly unstable in a dense medium, and captures a second electron in a nonequilibrium manner, Equation (68), with energy release $Q_{j}$, where $j$ is the label of the nonequilibrium process.

After the neutron-drip point $\left(\rho>\rho_{\mathrm{ND}} \simeq 6 \times 10^{11} \mathrm{~g} \mathrm{~cm}^{-3}\right)$, electron captures trigger neutron emissions,

$$
(A, Z)+e^{-} \longrightarrow(A, Z-1)+\nu_{e},
$$




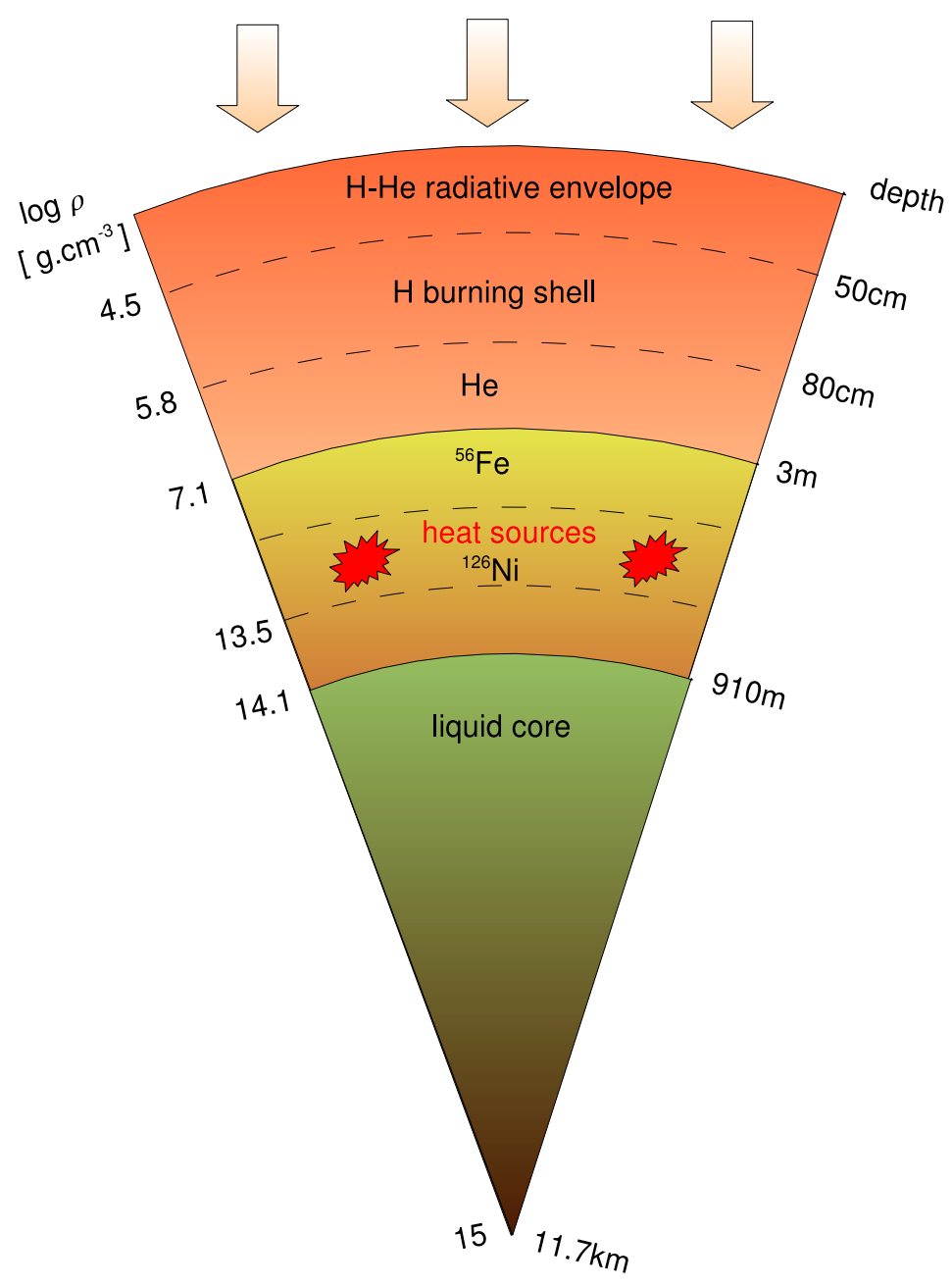

Figure 19: Model of an accreting neutron star crust. The total mass of the star is $M=1.4 M_{\odot}$. Stable hydrogen burning takes place in the H-burning shell, and produces helium, which accumulates in the Heshell. Helium ignites at $\rho \sim 10^{7} \mathrm{~g} \mathrm{~cm}^{-3}$, leading to a helium flash and explosive burning of all matter above the bottom of the He-layer into ${ }^{56} \mathrm{Ni}$, which captures electrons to become ${ }^{56} \mathrm{Fe}$. After $\sim 1 \mathrm{~h}$, the cycle of accretion, burning of hydrogen and explosion triggered by a helium flash repeats again and the layer of iron from the previous burst is pushed down. Based on the unpublished results of calculations by P. Haensel and J.L. Zdunik. Accreted crust model of [186, 185]. The core model of [125]. 


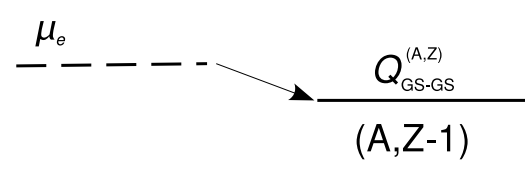

$$
e+(A, Z) \rightarrow(A, Z-1)+v_{e}
$$

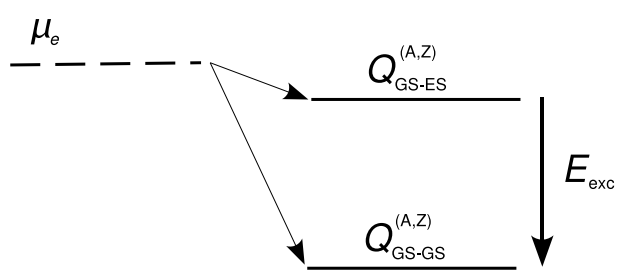

$(\mathrm{A}, \mathrm{Z}-2)$

$$
\begin{aligned}
& e+(A, Z-1) \rightarrow(A, Z-2)_{\mathrm{ES}}+\nu_{e} \\
& e+(A, Z-1) \rightarrow(A, Z-2)+\nu_{\mathrm{GS}}
\end{aligned}
$$

Figure 20: Electron capture processes.
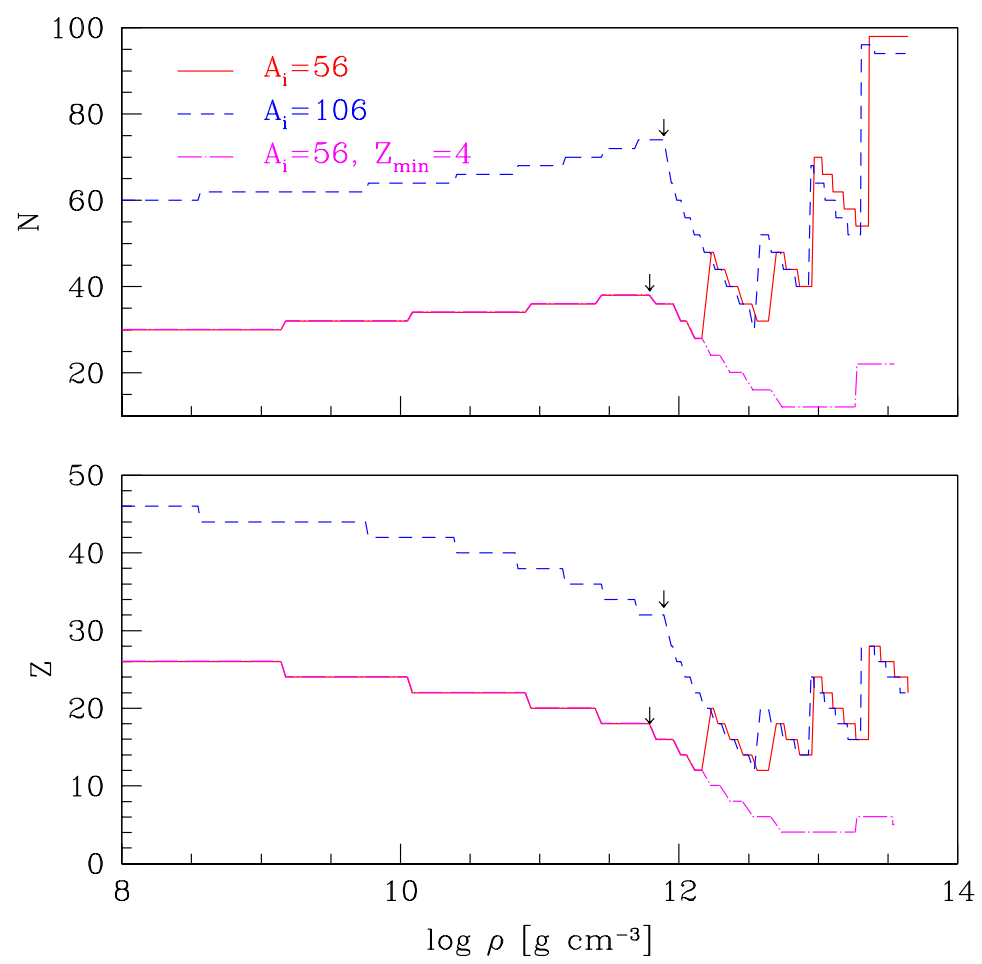

Figure 21: $Z$ and $N$ of nuclei vs. matter density in an accreted crust, for different models of dense matter. Solid line: $A_{\mathrm{i}}=106$; dotted line: $A_{\mathrm{i}}=56$. Every change of $N$ and $Z$, which takes place at a constant pressure, is accompanied by a jump in density: it is represented by small steep (but not perpendicular!) segments of the curves. These segments connect the top and the bottom density of a thin reaction shell. Arrows indicate positions of the neutron drip point. From Haensel \& Zdunik [187]. 


$$
(A, Z-1)+e^{-} \longrightarrow(A-\mathrm{k}, Z-2)+\mathrm{k} n+\nu_{e}+Q_{j}
$$

Due to the electron captures, the value of $Z$ decreases with increasing density. In consequence, the Coulomb barrier prohibiting the nucleus-nucleus reaction lowers. This effect, combined with the decrease of the mean distance between the neighboring nuclei, and a simultaneous increase of energy of the quantum zero-point vibrations around the nuclear lattice sites, opens up a possibility for the pycnonuclear fusion associated with quantum-mechanical tunneling through the Coulomb barrier due to zero-point vibrations (see, e.g., Section 3.7 of the book by Shapiro \& Teukolsky [373]). The pycnonuclear fusion timescale $\tau_{\text {pyc }}$ is a very sensitive function of $Z$. The chain of the reactions (69) and (70) leads to an abrupt decrease of $\tau_{\text {pyc }}$ typically by 7 to 10 orders of magnitude. In the onecomponent plasma approximation, the accreted crust is composed of spherical shells containing a single nuclide $(A, Z)$. Pycnonuclear fusion switches on as soon as $\tau_{\text {pyc }}$ is smaller than the time of the travel of a piece of matter (due to accretion) through the considered shell of mass $M_{\text {shell }}(A, Z)$, $\tau_{\text {acc }} \equiv M_{\text {shell }} / \dot{M}$. The masses of the shells are on the order of $10^{-5} M_{\odot}$. As a result, in the inner crust the chain of reactions (69 and 70) in several cases is followed by the pycnonuclear reaction, occurring on a timescale much shorter than $\tau_{\text {acc }}$. Introducing $Z^{\prime}=Z-2$, we then have

$$
\begin{gathered}
\left(A, Z^{\prime}\right)+\left(A, Z^{\prime}\right) \longrightarrow\left(2 A, 2 Z^{\prime}\right)+Q_{j, 1}, \\
\left(2 A, 2 Z^{\prime}\right) \longrightarrow\left(2 A-\mathrm{k}^{\prime}, 2 Z^{\prime}\right)+\mathrm{k}^{\prime} n+Q_{j, 2}, \\
\ldots \ldots \longrightarrow \ldots \ldots+Q_{j, 3},
\end{gathered}
$$

where dots in Equation (73) denote an actual nonequilibrium process, usually following reaction (72). The total heat deposition in matter, resulting from a chain of reactions involving a pycnonuclear fusion, Equations (71), (72) and (73), is $Q_{j}=Q_{j, 1}+Q_{j, 2}+Q_{j, 3}$.

The composition of accreted neutron star crusts, obtained by Haensel \& Zdunik [187], is shown in Figure 21. These results describe crusts built of accreted and processed matter up to the density $5 \times 10^{13} \mathrm{~g} \mathrm{~cm}^{-3}$ (slightly before the crust-core interface). At a constant accretion rate $\dot{M}=\dot{M}_{-9} \times 10^{-9} M_{\odot} /$ yr this will take $\sim 10^{6} \mathrm{yr} / \dot{M}_{-9}$. During that time, a shell of X-ray burst ashes will be compressed from $\sim 10^{8} \mathrm{~g} \mathrm{~cm}^{-3}$ to $\sim 10^{13} \mathrm{~g} \mathrm{~cm}^{-3}$.

Two different compositions of X-ray burst ashes at $\lesssim 10^{8} \mathrm{~g} \mathrm{~cm}^{-3}, A_{\mathrm{i}}, Z_{\mathrm{i}}$, were assumed. In the first case, $A_{\mathrm{i}}=56, Z_{\mathrm{i}}=26$, which is a "standard composition". In the second scenario $A_{\mathrm{i}}=106$, to imitate nuclear ashes obtained by Schatz et al. [364]. The value of $Z_{\mathrm{i}}=46$ stems then from the condition of beta equilibrium at $\rho=10^{8} \mathrm{~g} \mathrm{~cm}^{-3}$. As we see in Figure 21, after the pycnonuclear fusion region is reached, both curves converge (as explained in Haensel \& Zdunik [187], this results from $A_{\mathrm{i}}$ and $Z_{\mathrm{i}}$ in two scenarios).

\subsection{Deep crustal heating}

A neutron star crust that is not in full thermodynamic equilibrium constitutes a reservoir of energy, which can then be released during the star's evolution. The formation and structure of nonequilibrium neutron star crusts has been considered by many authors $[410,50,362,186,187$, 188, 178]. Such a crust can be produced by accretion onto a neutron star in compact LMXB, where the original crust built of a catalyzed matter (see Section 3) is replaced by a crust with a composition strongly deviating from that of nuclear equilibrium. However, building up the accreted crust takes time. The outer crust (Section 3.1), containing $\sim 10^{-5} M_{\odot}$, is replaced by the accreted crust in $\left(10^{4} / \dot{M}_{-9}\right)$ y. To replace the whole crust of mass $\sim 10^{-2} M_{\odot}$ by accreted matter requires $\left(10^{7} / \dot{M}_{-9}\right)$ y. After that time has passed, the entire "old crust" is pushed down through the crust-core interface, and is molten into the liquid core. The time $\left(10^{7} / \dot{M}_{-9}\right)$ y may seem huge.

However, LMXBs can live for $\sim 10^{9} \mathrm{y}$, so that a fully accreted crust on a neutron star is a realistic possibility.

Living Reviews in Relativity

http://www . livingreviews . org//rr-2008-10 
Heating due to nonequilibrium nuclear processes in the outer and inner crust of an accreting neutron star (deep crustal heating) was calculated, using different scenarios and models [186, 187, 188]. The effect of crustal heating on the thermal structure of the interior of an accreting neutron star can be seen in Figures 25 and 26. In what follows, we will describe the most recent calculations of crustal heating by Haensel \& Zdunik [188]. In spite of the model's simplicity (one-component plasma, $T=0$ approximation), the heating in the accreted outer crust obtained by Haensel \& Zdunik [188] agrees nicely with extensive calculations carried out by Gupta et al. [178]. The latter authors considered a multicomponent plasma, a reaction network of many nuclides, and included the contribution from the nuclear excited states. They found that electron captures in the outer crust proceed mostly via the excited states of the daughter nuclei, which then de-excite, the excitation energy heating the matter; this strongly reduces neutrino losses, accompanying nonequilibrium electron captures. The total deep crustal heating obtained by Haensel \& Zdunik [188] is equal to $Q_{\mathrm{tot}}=1.5$ and $1.9 \mathrm{MeV}$ per accreted nucleon for $A_{\mathrm{i}}=106$ and $A_{\mathrm{i}}=56$, respectively.

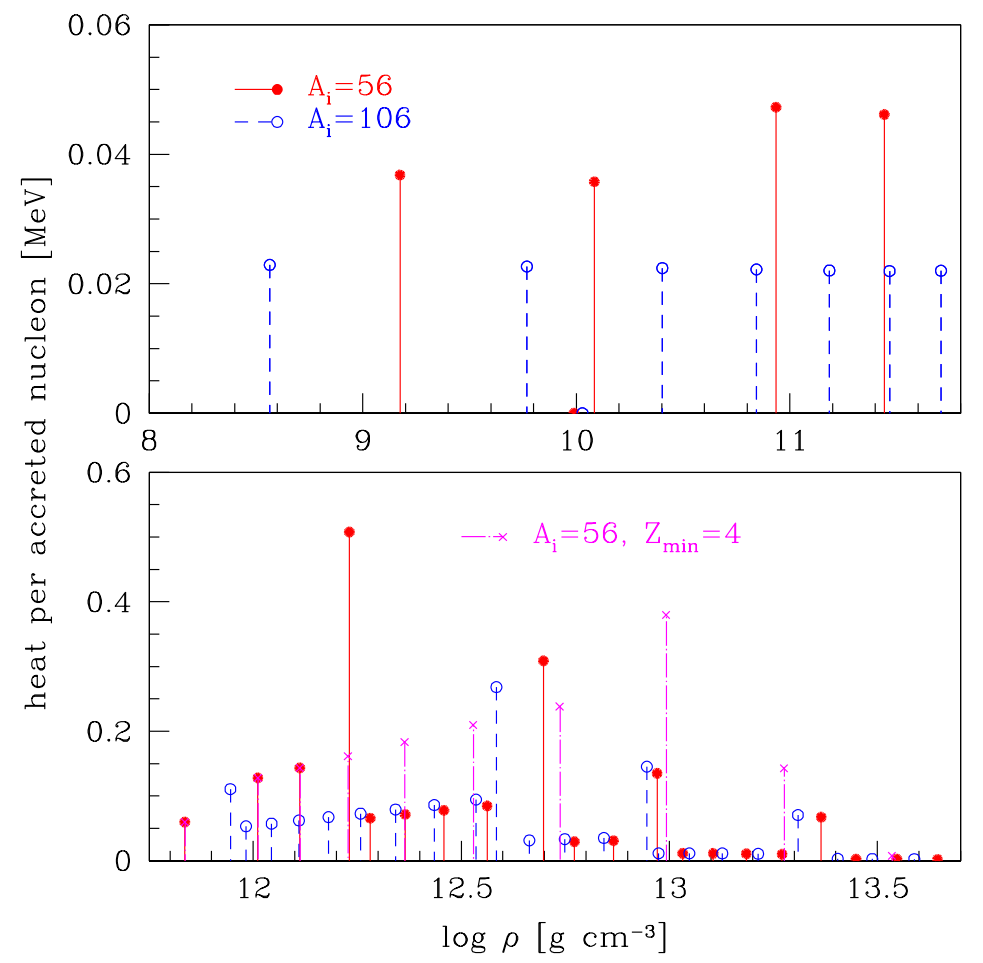

Figure 22: Heat sources accompanying accretion, in the outer (upper panel) and inner (lower panel) accreted crust. Vertical lines, positioned at the bottom of every reaction shell, represent the heat per accreted nucleon. Based on Haensel \& Zdunik [188]. Figure made by J.L. Zdunik.

In Figure 22, we show the heat deposited in the matter, per accreted nucleon, in the thin shells where nonequilibrium nuclear processes occur. Actually, reactions proceed at a constant pressure, and there is a density jump within a thin "reaction shell". The vertical lines, whose height gives the heat deposited in matter, are drawn at the density of the bottom of the reaction shell. The number of heat sources and the heating power of a single source depend on the assumed $A_{\mathrm{i}}$. In the case of $A_{\mathrm{i}}=56$ the number of sources is smaller, and their heat-per-nucleon values $Q_{j}$ are larger, than for $A_{\mathrm{i}}=106$.

An important quantity is the integrated heat deposited in the crust in the outer layer with 
bottom density $\rho$. It is given by

$$
Q^{(\alpha)}(\rho)=\sum_{j\left(\rho_{j}<\rho\right)} Q_{j}^{(\alpha)},
$$

where $(\alpha)$ labels the crustal heating model (specific $A_{\mathrm{i}}, Z_{\mathrm{i}}$, etc.). The quantity $Q^{(\alpha)}(\rho)$ for two models of compressional evolution is plotted in Figure 23. The second model illustrates the effect of switching off pycnonuclear reactions. This was done by artificially blocking pycnonuclear fusion until the nuclear charge went down to $Z_{\text {min }}=4$, which occurred at $\rho_{\text {pyc }}=10^{13.25} \mathrm{~g} \mathrm{~cm}^{-3}$. And yet, $Q^{(2)}$ for $\rho>10^{13} \mathrm{~g} \mathrm{~cm}^{-3}$ is very similar to that obtained in the first scenario, which was the most advantageous, as far as crust heating was concerned. Heating by pycnonuclear fusion at $\rho>10^{13} \mathrm{~g} \mathrm{~cm}^{-3}$ is insignificant. Heat from pycnonuclear fusions at $\rho \sim 10^{12} \mathrm{~g} \mathrm{~cm}^{-3}$ is to a large extent replaced by an additional heat release associated with electron captures and neutron emissions within the density range $10^{12}-10^{13} \mathrm{~g} \mathrm{~cm}^{-3}$. The values of $Q^{(1)}$ and $Q^{(2)}$ saturate above $10^{13.6} \mathrm{~g} \mathrm{~cm}^{-3}$, where $80 \%$ of nucleons are in neutron gas phase. All in all, for two scenarios with $A_{\mathrm{i}}=56$, the total deep crustal heat release is $1.8-1.9 \mathrm{MeV} /$ nucleon. For $A_{\mathrm{i}}=106$, these numbers are lowered by about $0.5 \mathrm{MeV} /$ nucleon.

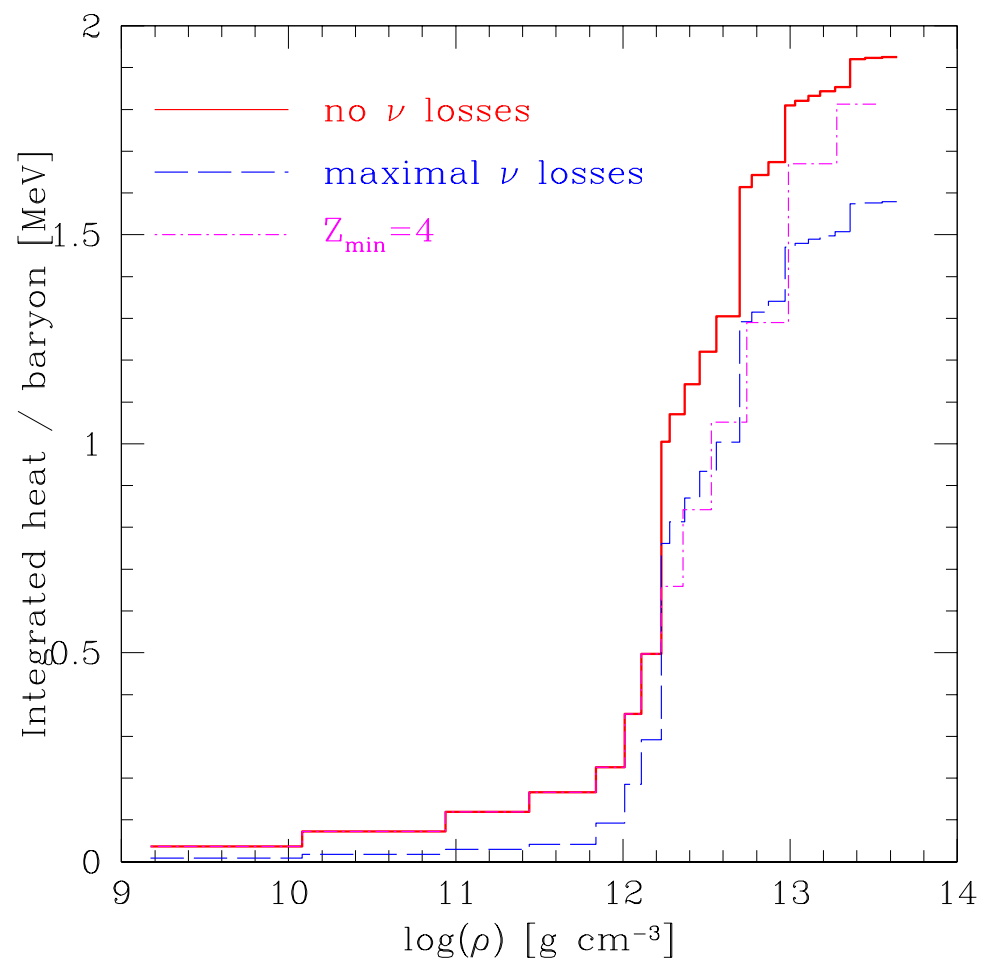

Figure 23: Integrated heat released in the crust, $Q(\rho)$ (per one accreted nucleon) versus $\rho$, assuming initial ashes of pure ${ }^{56} \mathrm{Fe}$. Solid line: HZ* model of Haensel \& Zdunik [188], with $A_{\mathrm{i}}=56$. Dash-dotted line: with pycnonuclear fusion blocked until $Z=Z_{\text {min }}=4$. Based on Haensel \& Zdunik [188]. Figure made by J.L. Zdunik.

The quite remarkable weak dependence of the total heat release in the crust, $Q_{\text {tot }}$, on the nuclear history of an element of matter undergoing compression from $\sim 10^{8} \mathrm{~g} \mathrm{~cm}^{-3}$ to $\sim 10^{13.6} \mathrm{~g} \mathrm{~cm}^{-3}$ deserves an explanation [188]. One has to study the most relevant thermodynamic quantity, the Gibbs free energy per nucleon (baryon chemical potential). Its minimum determines the 
state of thermodynamic equilibrium. Moreover, its drop at reaction surface $P=P_{j}$ yields the total energy release $Q_{j}$ per one nucleon [341]. In the $T=0$ approximation, we have $\mu_{\mathrm{b}}(P)=$ $[\mathcal{E}(P)+P] / n_{\mathrm{b}}(P)=$ enthalpy per nucleon. Minimizing $\mu_{\mathrm{b}}(P)$, at a fixed $P$, with respect to the independent thermodynamic variables $\left(A, Z\right.$, mean free neutron density $\bar{n}_{n}$, mean baryon density $n_{\mathrm{b}}$, size of the Wigner-Seitz cell, etc.), under the constraint of electro-neutrality, $\bar{n}_{p}=\bar{n}_{e}$, we get the ground state of the crust at a given $P$. This "cold catalyzed matter" (Section 3 ) corresponds to $\mu_{\mathrm{b}}^{(0)}(P)$. All other $\mu_{\mathrm{b}}^{(\alpha)}(P)$ curves displaying discontinuous drops due to nonequilibrium reactions included in a given evolutionary model $(\alpha)$ lie above the $\mu_{\mathrm{b}}^{(0)}(P)$; see Figure 24 .

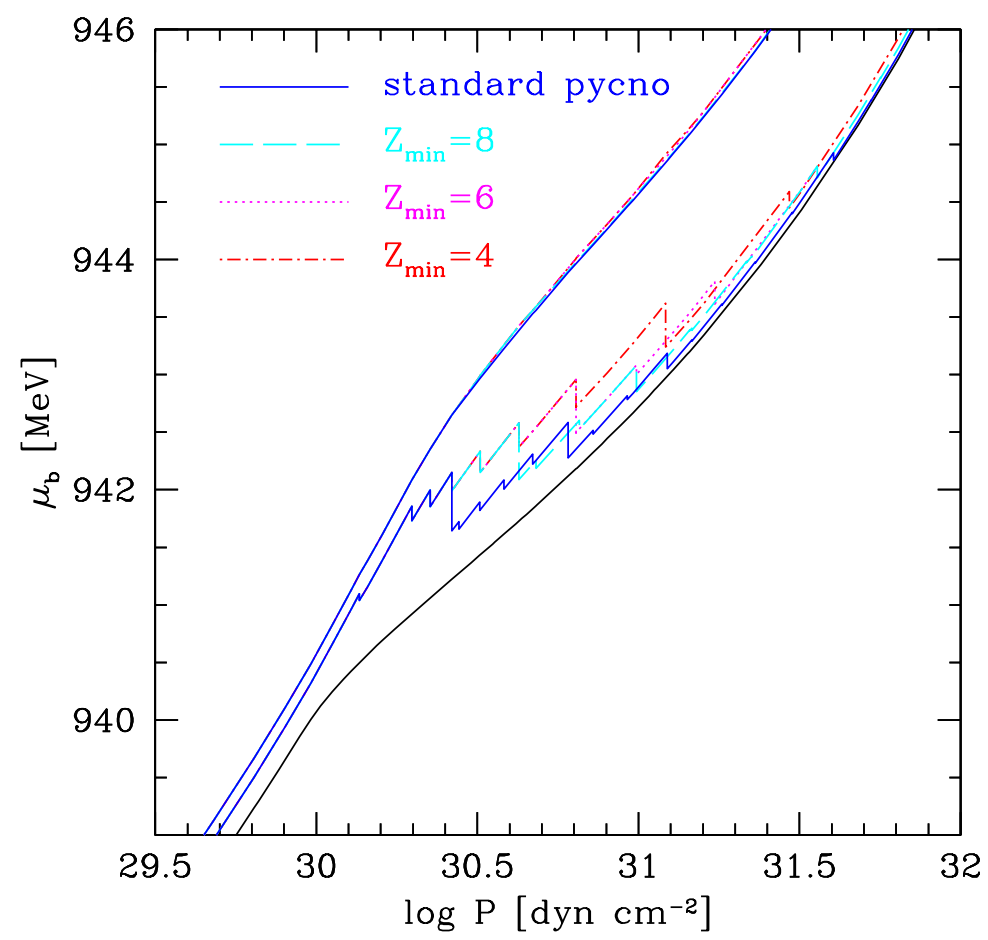

Figure 24: Baryon chemical potential at $T=0, \mu_{\mathrm{b}}=(\mathcal{E}+P) / n_{\mathrm{b}}$ vs. pressure for different models of neutron star crust. Black solid line: $\mu_{\mathrm{b}}^{(0)}(P)$ for the ground-state crust. Lines with discontinuous drops: $\mu_{\mathrm{b}}^{(\alpha)}(P)$ for four evolution models in the accreted crust with $A_{\mathrm{i}}=56$. Upper four smooth lines, which nearly coincide: $\mu_{\mathrm{b}}^{(\alpha)}(P)+\sum_{j\left(P<P_{j}\right)} Q_{j}^{(\alpha)} \approx \bar{\mu}_{\mathrm{b}}(P)$. For an explanation see the text. Based on Haensel \& Zdunik [188]. Figure made by J.L. Zdunik.

This makes visual the fact that noncatalyzed matter is a reservoir of energy, released in nonequilibrium processes that move the matter closer to the absolute ground state. In spite of dramatic differences between different $\mu_{\mathrm{b}}^{(\alpha)}(P)$ in the region where the bulk of the heating occurs, $P=\left(10^{30}-10^{31.5}\right) \mathrm{erg} \mathrm{cm}^{-3}$, the functions $\mu_{\mathrm{b}}^{(\alpha)}(P)$ tend to $\mu_{\mathrm{b}}^{(0)}(P)$ for $P \gtrsim 10^{32} \mathrm{erg} \mathrm{cm}^{-3}$. The general structure of different $\mu_{\mathrm{b}}^{(\alpha)}(P)$ is similar. At the same $P$, their continuous segments have nearly the same slope. What differs between $\mu_{\mathrm{b}}^{(\alpha)}(P) \mathrm{s}$ are discontinuous drops, by $Q_{j}^{(\alpha)}$, at reaction 
thresholds $P_{j}^{(\alpha)}$. The functions $\mu_{\mathrm{b}}^{(\alpha)}(P)$ can therefore be expressed as (see Haensel \& Zdunik [188])

$$
\mu_{\mathrm{b}}^{(\alpha)}(P) \approx \bar{\mu}_{\mathrm{b}}(P)-\sum_{j\left(P<P_{j}\right)} Q_{j}^{(\alpha)},
$$

where $\bar{\mu}_{\mathrm{b}}(P)$ is a smooth function of $P$, independent of $(\alpha)$. For $P>10^{33.5} \mathrm{erg} \mathrm{cm}^{-3}$ the values of $Q_{j}$ are negligibly small, and all $\mu_{\mathrm{b}}^{(\alpha)}(P)$ come quite close to the ground state line. This implies that the sum $\sum_{j} Q_{j}^{(\alpha)}$ must be essentially independent of $(\alpha)$.

\subsection{Thermal structure of accreted crusts and X-ray bursts}

A thermonuclear flash is triggered by an instability in the thermonuclear burning. The relevant quantities are the local heating rate due to thermonuclear fusion, $\dot{\varepsilon}_{\text {nuc }}$, and cooling rate, $\dot{\varepsilon}_{\text {cool }}$, resulting from heat diffusion, volume expansion, and neutrino emission. A steady state of an accreting neutron star corresponds to $\dot{\varepsilon}_{\text {nuc }}=\dot{\varepsilon}_{\text {cool }}$. It depends on the accretion rate, composition of accreted plasma, structure and physical properties (thermal conductivity, neutrino emissivity, etc. of the stellar interior. Examples of the steady thermal structure of accreting neutron stars are shown in Figures 25 and 26.

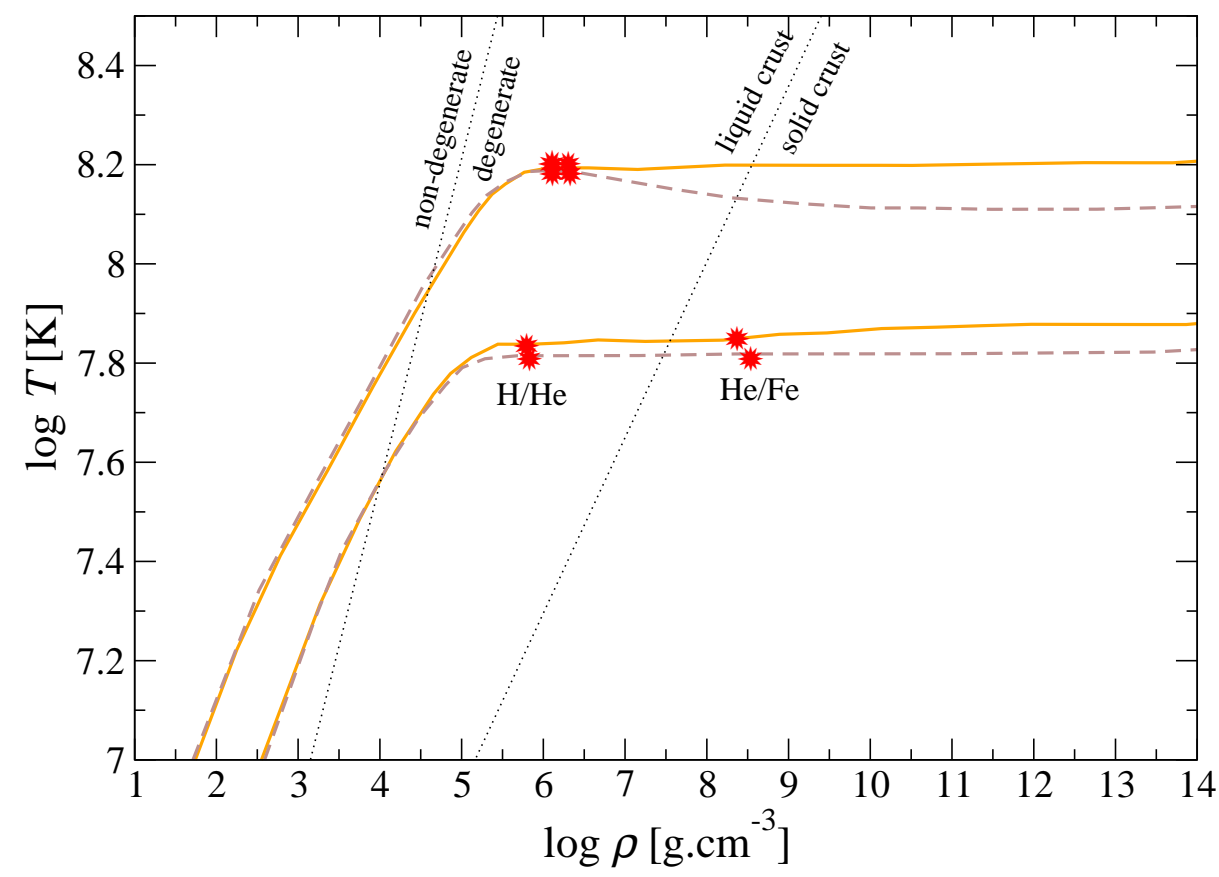

Figure 25: Temperature (local, in the reference frame of the star) vs. density within the crust of an accreting neutron star (soft EoS of the core, $M=1.24 M_{\odot}$ ) in a steady thermal state, with standard cooling of the core (no fast cooling of the direct Urca type). Upper solid curve $-\dot{M}=10^{-9.96} M_{\odot} \mathrm{y}^{-1}$. Lower solid curve $-\dot{M}=10^{-11} M_{\odot} \mathrm{y}^{-1}$. H and He burning shells are indicated by asterisks. Deep crustal heating is included. Dashed line - temperature profile without deep crustal heating. Based on Figure 3b of Miralda-Escudé el al. [292].

A stable steady state of an accreting neutron star satisfies

$$
\dot{\varepsilon}_{\text {nuc }}=\dot{\varepsilon}_{\text {cool }}, \frac{\partial \dot{\varepsilon}_{\text {nuc }}}{\partial T}<\frac{\partial \dot{\varepsilon}_{\text {cool }}}{\partial T} .
$$




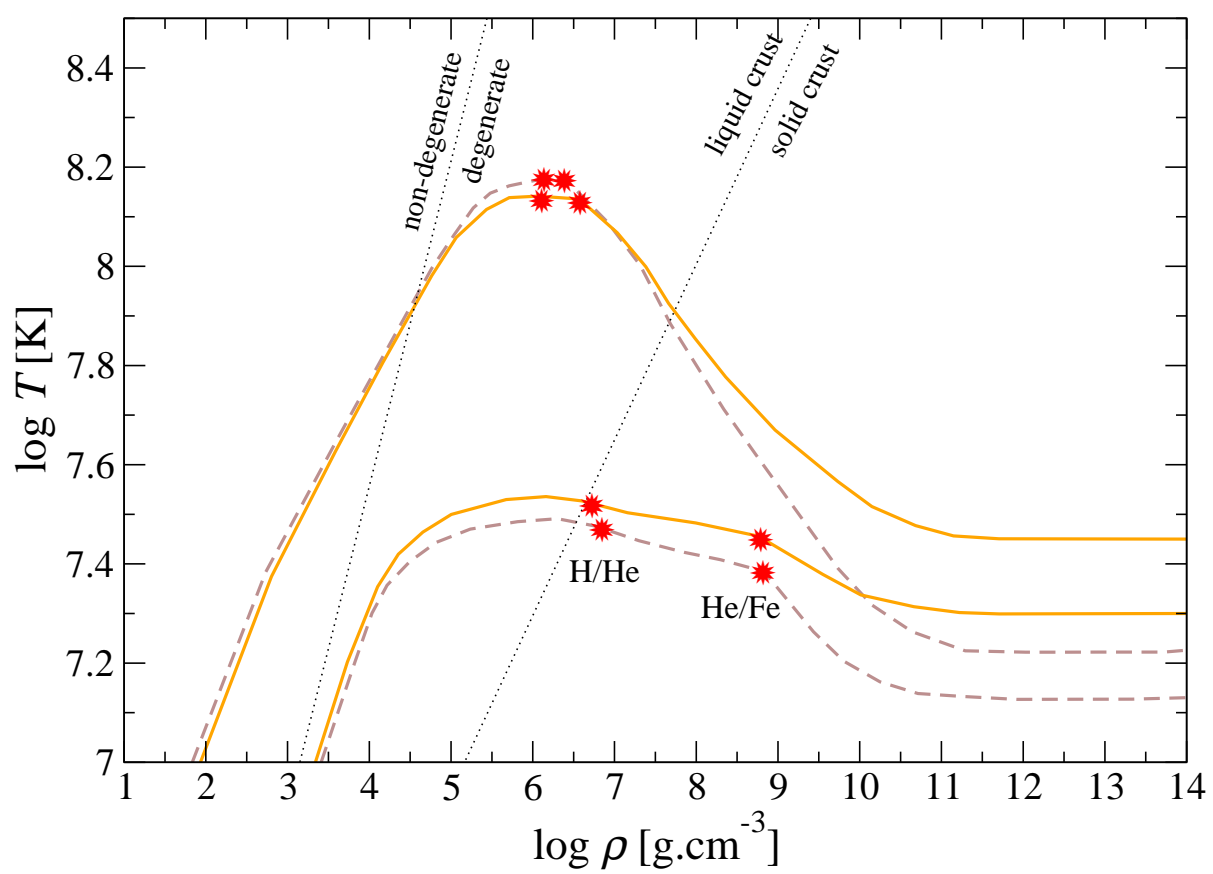

Figure 26: Same as in Figure 25, but for fast neutrino cooling due to pion condensation in the inner core. Based on Figure 3c of Miralda-Escudé et al. [292].

The inequality guarantees that any thermal perturbation will be damped by a self-regulated cooling. Under the increasing weight of accreted matter, an element of the crust in the He burning shell moves in the $\rho-T$ plane, in the direction of increasing $\rho$. At some moment, after crossing the "ignition line" characterized by

$$
\frac{\partial \dot{\varepsilon}_{\mathrm{nuc}}}{\partial T}=\frac{\partial \dot{\varepsilon}_{\mathrm{cool}}}{\partial T} \Longrightarrow \rho_{\mathrm{ign}}(T),
$$

burning becomes unstable, and a self-accelerating thermonuclear flash is ignited because

$$
\frac{\partial \dot{\varepsilon}_{\text {nuc }}}{\partial T}>\frac{\partial \dot{\varepsilon}_{\text {cool }}}{\partial T}
$$

In the standard picture, it is the instability in the helium burning via $3 \alpha \longrightarrow{ }^{12} \mathrm{C}$, which triggers an X-ray burst (see, e.g., [152]). The total energy release in a burst can then be easily estimated (neglecting the general relativistic correction) via

$$
E_{\mathrm{burst}} \approx Q_{\mathrm{nuc}} M_{\mathrm{burn}} / m_{\mathrm{u}}
$$

where $Q_{\text {nuc }}$ is the mean energy per nucleon released in the thermonuclear flash $(2-8 \mathrm{MeV}$, depending on composition of burnt material), $M_{\mathrm{burn}}=M_{\mathrm{env}}\left(\rho_{\mathrm{ign}}\right)$ is the mass of the envelope burnt in the flash (determined by the ignition density $\rho_{\mathrm{ign}}$ ), and $m_{\mathrm{u}}$ is the atomic mass unit. The value of $M_{\text {env }}\left(\rho_{\text {ign }}\right)$ can be read from Figure 39. A good model of X-ray bursts should yield $E_{\text {burst }} \sim 10^{40} \mathrm{erg}$ with a recurrence time of hours. This can be satisfied with ignition of helium flashes at $\sim 10^{7} \mathrm{~g} \mathrm{~cm}^{-3}$. 


\section{Equation of State}

In this section we discuss the Equation of State (EoS) of the neutron star crust. Three different cases will be considered: cold catalyzed matter in Section 5.1, accreted crust matter in Section 5.2 assuming the formation scenario described in Section 4, and hot dense matter in supernova cores in Section 5.4.

\subsection{Ground state crust}

The EoS of the outer crust in the ground state approximation (see Section 3.1) is rather well established, so that the pressure at any given density is determined within a few percent accuracy [42, 183, 357] as can be seen in Figure 27.

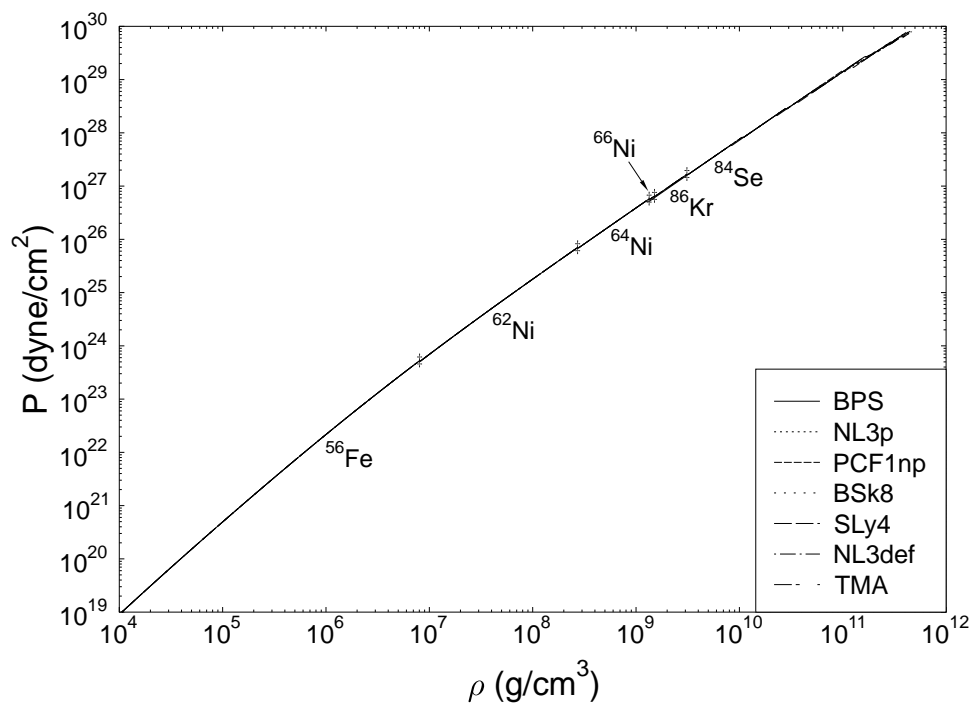

Figure 27: EoS of the ground state of the outer crust for various nuclear models. From Rüster et al. [357]. A zoomed-in segment of the EoS just before the neutron drip can be seen in Figure 28.

Uncertainties arise above the density $\rho>6 \times 10^{10} \mathrm{~g} \mathrm{~cm}^{-3}$ as shown in Figure 28 because experimental data are lacking. However, one may hope that with the improvement of experimental techniques, experimental data on very exotic nuclei will become available in the future.

On the contrary, the inner crust nuclei cannot be studied in a laboratory because their properties are influenced by the gas of dripped neutrons, as reviewed in Section 3.2. This means that only theoretical models can be used there and consequently the EoS after neutron drip is much more uncertain than in the outer layers. The neutron gas contributes more and more to the total pressure with increasing density. Therefore, the problem of correct modeling of the EoS of a pure neutron gas at subnuclear densities becomes important. The true EoS of cold catalyzed matter stems from a true nucleon Hamiltonian, expected to describe nucleon interactions at $\rho \lesssim \rho_{0}$, where $\rho_{0}$ is the nuclear saturation density. To make the solution of the many-body problem feasible, the task is reduced to finding an effective nucleon Hamiltonian, which would enable one to calculate reliably both the properties of laboratory nuclei and the EoS of cold catalyzed matter for $10^{11} \mathrm{~g} \mathrm{~cm}^{-3} \lesssim \rho \lesssim \rho_{0}$. The task also includes the calculation of the crust-core transition. We 


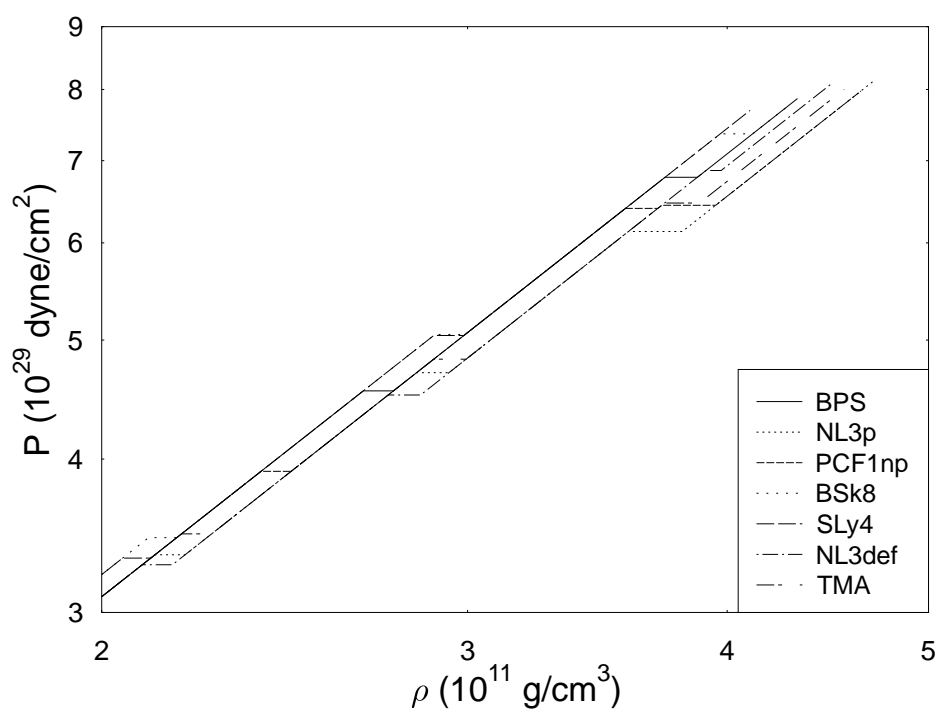

Figure 28: EoS of the ground state of the outer crust just before neutron drip for various nuclear models. From Rüster et al. [357]

will illustrate the general results with two examples of the EoS of the inner crust, calculated in the compressible-liquid-drop model (see Section 3.2.1) using the effective nucleon-nucleon interactions FPS (Friedman-Panharipande-Skyrme [320]) and SLy (Skyrme-Lyon [86, 88, 87]).

As one can see in Figure 29, significant differences between the SLy and FPS EoS are restricted to the density interval $4 \times 10^{11}-4 \times 10^{12} \mathrm{~g} \mathrm{~cm}^{-3}$. They result mainly from the fact that the density at which neutron drip occurs for each is different: $\rho_{\mathrm{ND}}(\mathrm{SLy}) \simeq 4 \times 10^{11} \mathrm{~g} \mathrm{~cm}^{-3}$ (in good agreement with the "empirical EoS" of Haensel \& Pichon [183]), while $\rho_{\mathrm{ND}}(\mathrm{FPS}) \simeq 6 \times 10^{11} \mathrm{~g} \mathrm{~cm}^{-3}$. For $4 \times 10^{12} \mathrm{~g} \mathrm{~cm}^{-3} \lesssim \rho \lesssim 10^{14} \mathrm{~g} \mathrm{~cm}^{-3}$ the SLy and FPS EoSs are very similar, with the FPS EoS being a little softer at the highest densities considered. The detailed behavior of the two EoSs near the crust-core transition can be seen in Figure 30. The FPS EoS is softer there than the SLy EoS (for pure neutron matter the FPS model is softer at subnuclear densities; see [184]).

In the case of the SLy EoS, the crust-liquid core transition takes place as a very weak first-order phase transition, with a relative density jump on the order of one percent. Notice that, for this model, spherical nuclei persist to the very bottom of the crust [126]. As seen from Figure 30, the crust-core transition is accompanied by a noticeable stiffening of the EoS. For the FPS EoS the situation is different. Namely, the crust-core transition takes place through a sequence of phase transitions with changes of nuclear shapes as discussed in Section 3.3. These phase transitions make the crust-core transition smoother than in the SLy case, with a gradual increase of stiffness (see Figure 35). While the presence of exotic nuclear shapes is expected to have dramatic consequences for the transport, neutrino emission, and elastic properties of neutron star matter, their effect on the EoS is rather small.

The overall SLy EoS of the crust, calculated including adjacent segments of the liquid core and the outer crust, is shown in Figure 33. In the outer crust segment, the SLy EoS cannot be visually distinguished from the EoSs of Haensel \& Pichon [183] or Rüster et al. [357], which are based on experimental nuclear masses. An important dimensionless parameter, measuring the stiffness of 


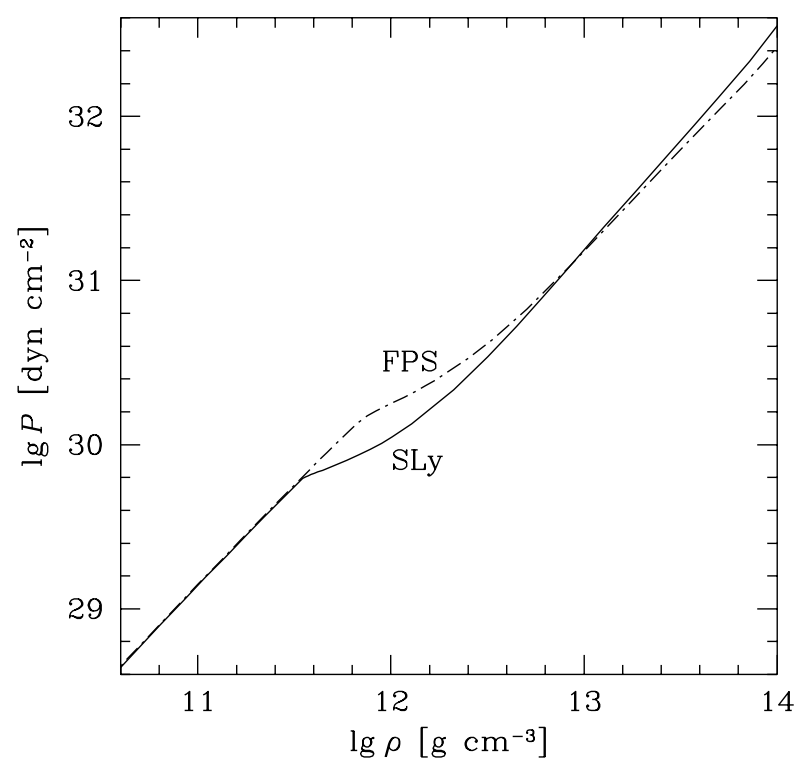

Figure 29: Comparison of the SLy and FPS EoSs. From [184].

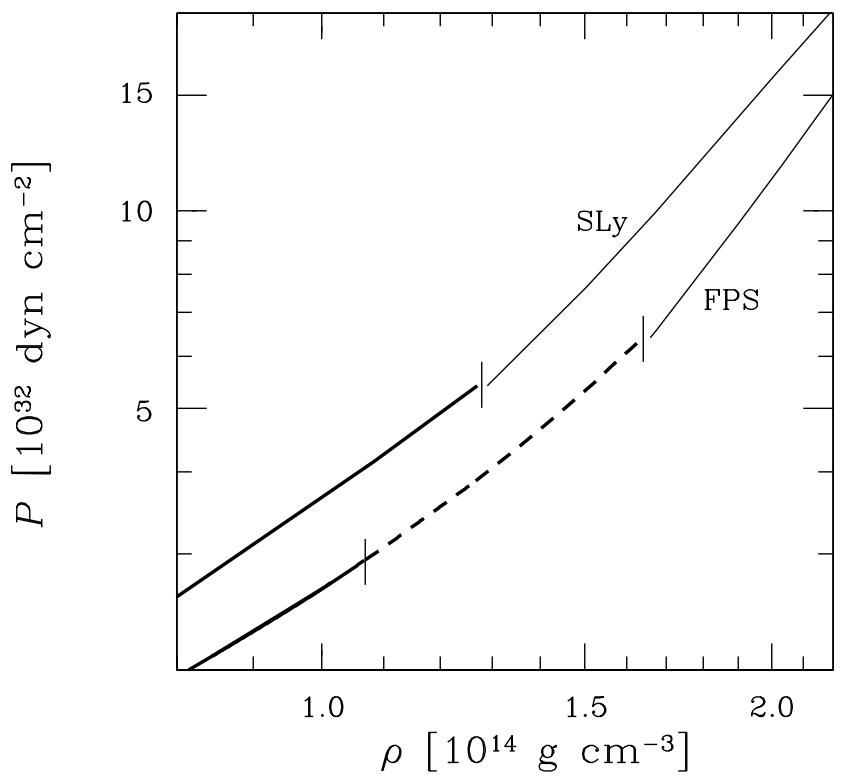

Figure 30: Comparison of the SLy and FPS EoSs near the crust-core transition. Thick solid line: inner crust with spherical nuclei. Dashed line corresponds to "exotic nuclear shapes". Thin solid line: uniform npe matter. From [184]. 


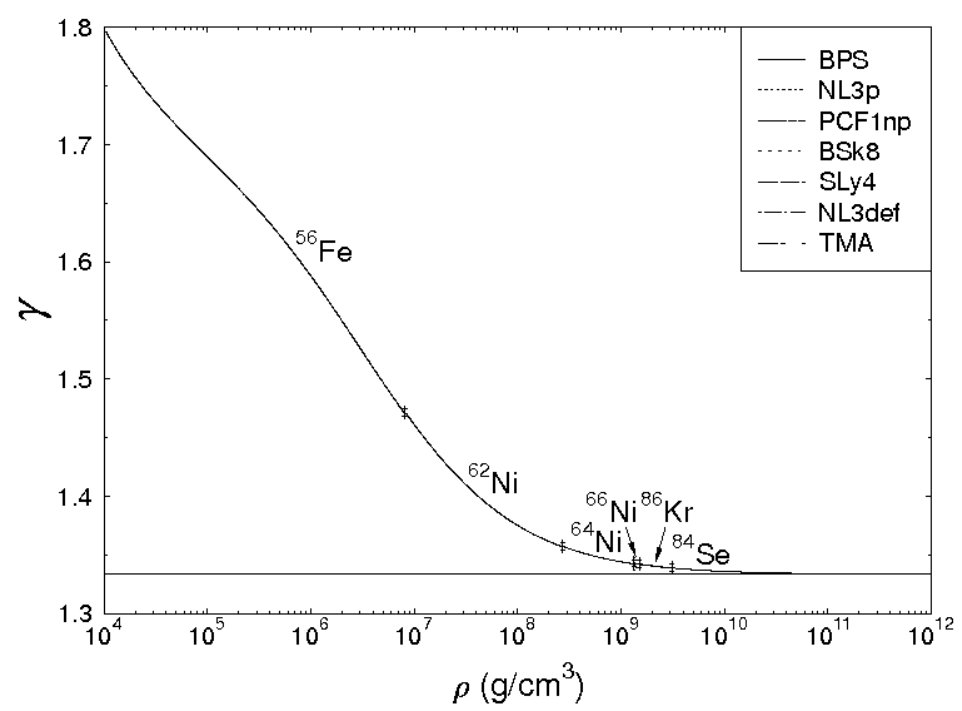

Figure 31: Adiabatic index $\gamma$ for various EoSs of the ground-state outer crust below neutron drip. The horizontal line corresponds to $\gamma=4 / 3$. The neutron drip point $\rho_{\mathrm{ND}} \approx 4 \times 10^{11} \mathrm{~g} \mathrm{~cm}^{-3}$ depends slightly on the EoS model used and, therefore, is not marked. From Rüster et al. [357].

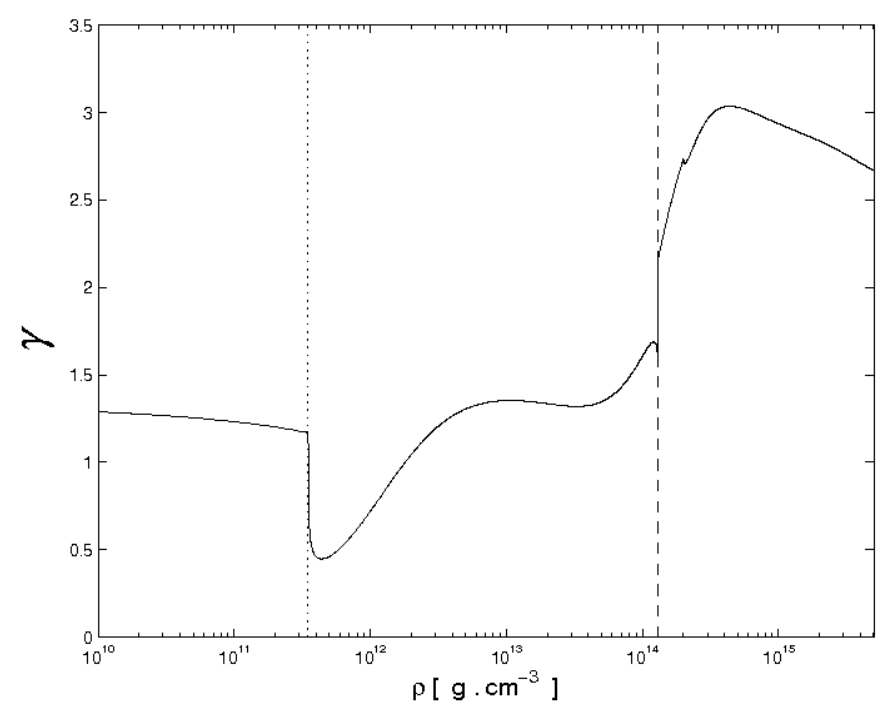

Figure 32: Adiabatic index $\gamma$ for the EoS of the ground-state crust. Dotted vertical lines correspond to the neutron drip and crust-core interface points. Calculations performed using the SLy EoS of Douchin \& Haensel [125]. 


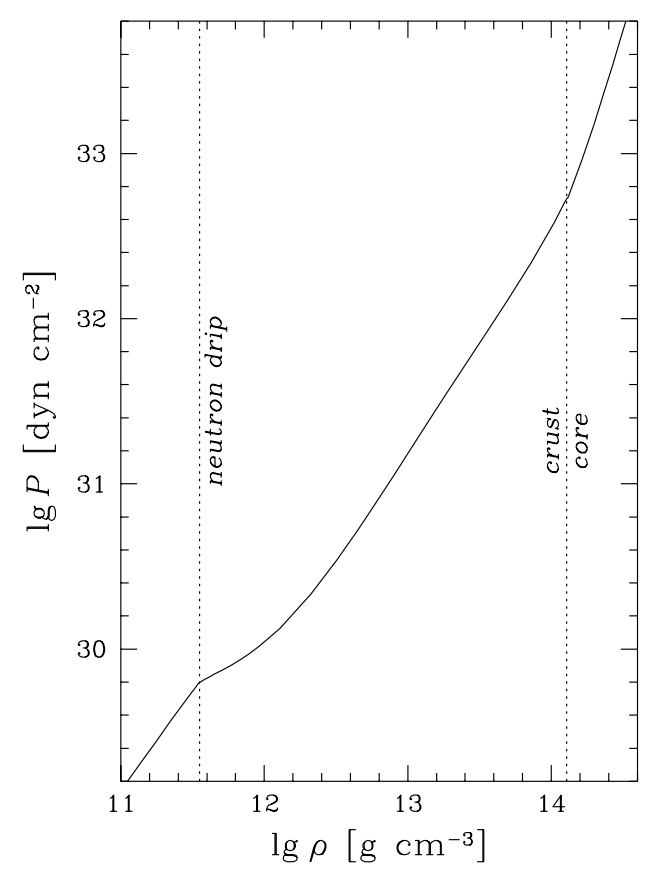

Figure 33: The SLy EoS. Dotted vertical lines correspond to the neutron drip and crust-core transition. From [184].

an EoS at a given density, is the adiabatic index,

$$
\gamma=\frac{\mathrm{d} \log P}{\mathrm{~d} \log n_{\mathrm{b}}}=\frac{n_{\mathrm{b}}}{P} \frac{\mathrm{d} P}{\mathrm{~d} n_{\mathrm{b}}},
$$

which at subnuclear density can be approximated by $\gamma \simeq(\rho / P) \mathrm{d} P / \mathrm{d} \rho$. The total pressure $P$ is defined by Equation (24). The adiabatic index $\gamma$ is shown in Figures 31 and 32 as a function of mass density $\rho$. At $\rho_{\mathrm{ND}}>\rho \gtrsim 10^{9} \mathrm{~g} \mathrm{~cm}^{-3}$, we have $\gamma \approx 4 / 3$. This is because in these outer crust layers, pressure is very well approximated by the sum of the contribution of the ultrarelativistic electron gas $\left(P_{e}\right)$ and of the lattice contribution $\left(P_{L}\right)$, which both have the same density dependence $\propto \rho^{4 / 3}$ (see Section 3.1). One notices in Figure 32 a dramatic softening in the density region following the neutron drip point, $\rho \gtrsim \rho_{\mathrm{ND}}$. This means, in particular, that no stable stars can exist with central densities around $\rho_{\mathrm{ND}}$ because the compressibility of the matter is too low. Then the EoS stiffens gradually, with a significant increase of $\gamma$ near the crust-core interface. A jump in $\gamma$ on the core side is connected with the disappearance of nuclei, and a subsequent stiffening (due to the nucleon-nucleon interaction) in the uniform npe liquid. Figures 31 and 32 show that the EoS of the inner crust is very different from the polytropic form $P \propto \rho^{\gamma_{0}}$, where $\gamma_{0}$ is a constant. Tabulated and analytical EoSs of the ground state crust are available online [210, 118].

\subsection{Accreted crust}

A model of the EoS of accreted crusts was calculated by Haensel \& Zdunik [185]. They used the compressible liquid drop model (see Section 3.2.1) with a "single nucleus" scenario.

In Figure 34 this EoS is compared with the SLy model of cold catalyzed matter described in Section 5.1. At $\rho<\rho_{\mathrm{ND}}$ both EoSs are very similar. The reason is that for $\rho<\rho_{\mathrm{ND}}$, as discussed in Section 3.1, we have $P \simeq P_{e}\left(n_{e}\right)$ with $n_{e}=(Z / A) n_{\mathrm{b}}$ and the ratio $Z / A$ is quite 
similar for both accreted and ground state crusts. Large differences appear for $\rho_{\mathrm{ND}} \lesssim \rho \lesssim 10 \rho_{\mathrm{ND}}$, where the EoS of accreted matter is stiffer than that of cold catalyzed matter. One also notices well-pronounced density jumps at constant pressure in the EoS of accreted matter. They are associated with discontinuous changes in nuclear composition, an artifact of the one-component plasma approximation. The jumps are particularly large for $\rho_{\mathrm{ND}} \lesssim \rho \lesssim 10 \rho_{\mathrm{ND}}$.
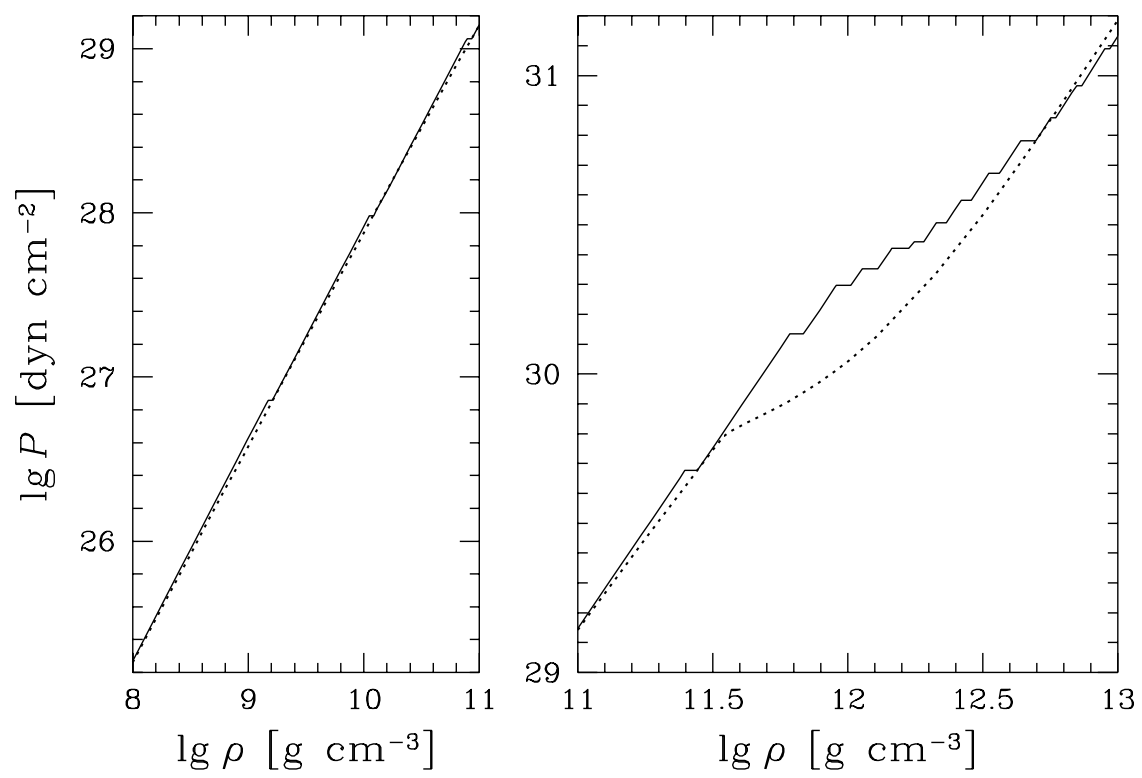

Figure 34: Comparison of the SLy EoS for cold catalyzed matter (dotted line) and the EoS of accreted crust (solid line). Figure by A.Y. Potekhin.

The difference between the cold catalyzed and accreted matter EoSs decreases for large density. Both curves are very close to each other for $\rho>10^{13} \mathrm{~g} \mathrm{~cm}^{-3}$. This is because for such a high density the pressure is mainly produced by the neutron gas and is not sensitive to the detailed composition of the nuclear clusters. In view of this, one can use the EoS of the catalyzed matter for calculating the hydrostatic equilibrium of the high-density $\left(\rho>10^{13} \mathrm{~g} \mathrm{~cm}^{-3}\right)$ internal layer of the accreted crust.

\subsection{Effect of magnetic fields on the EoS}

Typical values of the surface magnetic field of radio pulsars are $B \sim 10^{12} \mathrm{~g}$. For magnetars, surface magnetic fields can be as high as $\sim 10^{15} \mathrm{G}$. Effects of such fields on the EoS and structure of the crust are briefly reviewed in Section 6.4. A detailed study of the effect of $\boldsymbol{B}$ on neutron star envelopes can be found in Chapter 4 of [184].

\subsection{Supernova core at subnuclear density}

The outer layers of the supernova core, which after a successful explosion will become the envelope of a proto-neutron star, display a similar range of densities $\rho \lesssim 10^{14} \mathrm{~g} \mathrm{~cm}^{-3}$ and are governed by the same nuclear Hamiltonian as the neutron star crust. This is why we include it in the present review. 
The subnuclear density layer of supernova cores shows many similarities with that of neutron star crusts. In both cases, the matter is formed of nuclear clusters embedded in a sea of leptons and hadrons. Nevertheless, the conditions are very different since supernova cores are lepton rich (contain many electrons, positrons as well as trapped neutrinos and antineutrinos) with lepton fraction

$$
Y_{\mathrm{L}}=\frac{n_{e^{-}}+n_{\nu_{e}}-n_{e^{+}}-n_{\bar{\nu}_{e}}}{n_{b}} \lesssim 0.4
$$

and very hot with temperatures typically $T \lesssim 40 \mathrm{MeV} / k_{\mathrm{B}}$ while the matter in neutron star crusts is cold, neutrino free, and in $\beta$-equilibrium. Neutrinos remain trapped with the supernova core for several seconds, which is their diffusion timescale in dense hot matter. The presence of nuclei in dense hot matter is of utmost importance for the neutrino opacity of the supernova core. Namely, the scattering cross section of neutrinos off nuclei of mass number $A$ is $A$ times larger than the sum of the individual cross sections for $A$ nucleons in a nucleon gas (see, e.g., [373]). As long as neutrinos are trapped, $Y_{L}=$ const., and collapse is adiabatic.

Of particular importance for supernova simulations is the adiabatic index defined by

$$
\gamma=\left.\frac{\partial \log P}{\partial \log \rho}\right|_{s, Y_{e}},
$$

where $s$ is the entropy per nucleon in units of $k_{\mathrm{B}}$ and $Y_{e}$ is the electron fraction. For low entropies per nucleon on the order of the Boltzmann constant $k_{\mathrm{B}}$, and for densities $\rho \lesssim 5 \times 10^{13} \mathrm{~g} \mathrm{~cm}^{-3}$ the value of $\gamma$ is mainly determined by the relativistic electrons and is thus close to $4 / 3$ (adiabatic index of an ultra-relativistic Fermi gas); see Figure 35. The adiabatic index jumps to larger values when the nuclear clusters dissolve into a uniform mixture of nucleons and leptons, as discussed in Section 5.1. The adiabatic index is shown in Figure 35 for three different EoS that have been used in supernova simulations. The impact of the presence of nuclear pasta phases (Section 3.3) on the adiabatic index is illustrated in Figure 36.

The striking differences between the adiabatic index of supernova matter, $\gamma_{\mathrm{SN}}(\rho)$, and that for cold catalyzed matter in neutron stars, $\gamma_{\mathrm{NS}}(\rho)$, deserves additional explanation. In the core collapse, compression of the matter becomes adiabatic as soon as $\rho \gtrsim 10^{11} \mathrm{~g} \mathrm{~cm}^{-3}$, so that the entropy per nucleon $s=$ const. Simultaneously, due to neutrino trapping, the electron-lepton fraction is frozen, $Y_{\mathrm{L}}=$ const. The condition $s=$ const. $\approx 1 k_{\mathrm{B}}$ blocks evaporation of nucleons from the nuclei; the motion of nucleons have to remain ordered. Therefore, the fraction of free nucleons stays small and they do not contribute significantly to the pressure, which is supplied by the electrons, until the density reaches $10^{14} \mathrm{~g} \mathrm{~cm}^{-3}$.

At $\rho \gtrsim 10^{14} \mathrm{~g} \mathrm{~cm}^{-3}$, nuclei coalesce forming uniform nuclear matter. Thus, there are two density regimes for $\gamma_{\mathrm{SN}}(\rho)$. For $\rho \lesssim 10^{14} \mathrm{~g} \mathrm{~cm}^{-3}$, pressure is supplied by the electrons, while nucleons are confined to the nuclei, so that $\gamma_{\mathrm{SN}} \simeq 4 / 3 \approx 1.3$. Then, for $\rho \gtrsim 10^{14} \mathrm{~g} \mathrm{~cm}^{-3}$ nuclei coalesce into uniform nuclear matter, and the supernova matter stiffens violently, with the adiabatic index jumping by a factor of about two, to $\gamma_{\mathrm{SN}} \approx 2-3$. This stiffening is actually responsible for the bounce of infalling matter. An additional factor stabilizing nuclei at $\rho \lesssim 10^{14} \mathrm{~g} \mathrm{~cm}^{-3}$ in spite of a high $T>10^{10} \mathrm{~K}$, is a large lepton fraction, $Y_{\mathrm{L}} \approx 0.4$, enforcing a relatively large proton fraction, $Y_{p}^{\mathrm{SN}} \approx 0.3$, to be compared with $Y_{p}^{\mathrm{NS}} \approx 0.05$ for neutron stars.

Finally, for supernova matter we notice the absence of a neutron-drip softening, so well pronounced in $\gamma_{\mathrm{NS}}$, Figure 31. This is because neutron gas is present in supernova matter also at $\rho<10^{11} \mathrm{~g} \mathrm{~cm}^{-3}$, and the increase of the free neutron fraction at higher density is prevented by strong neutron binding in the nuclei (large $Y_{p}^{\mathrm{SN}}$ ), and low $s \approx 1 k_{\mathrm{B}}$. 


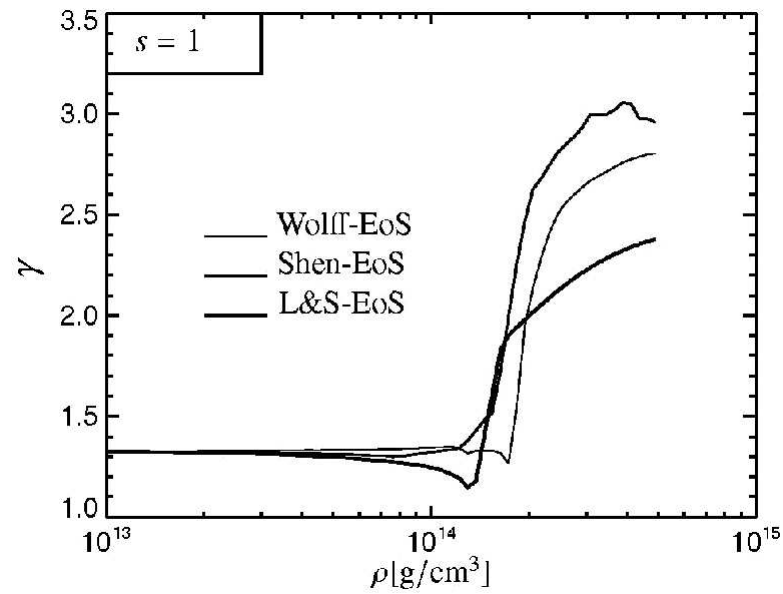

Figure 35: Variation of the adiabatic index $\gamma$ of supernova matter with mass density $\rho$ for three different EoSs with trapped neutrinos: Lattimer and Swesty [255] (compressible liquid drop model), Shen [374, 375] (relativistic mean field theory in the local density approximation) and Wolff [197] (Hartree-Fock with Skyrme nucleon-nucleon interaction). The lepton fraction is $Y_{L}=0.4$ and the entropy per nucleon is equal to $1 k_{\mathrm{B}}$.

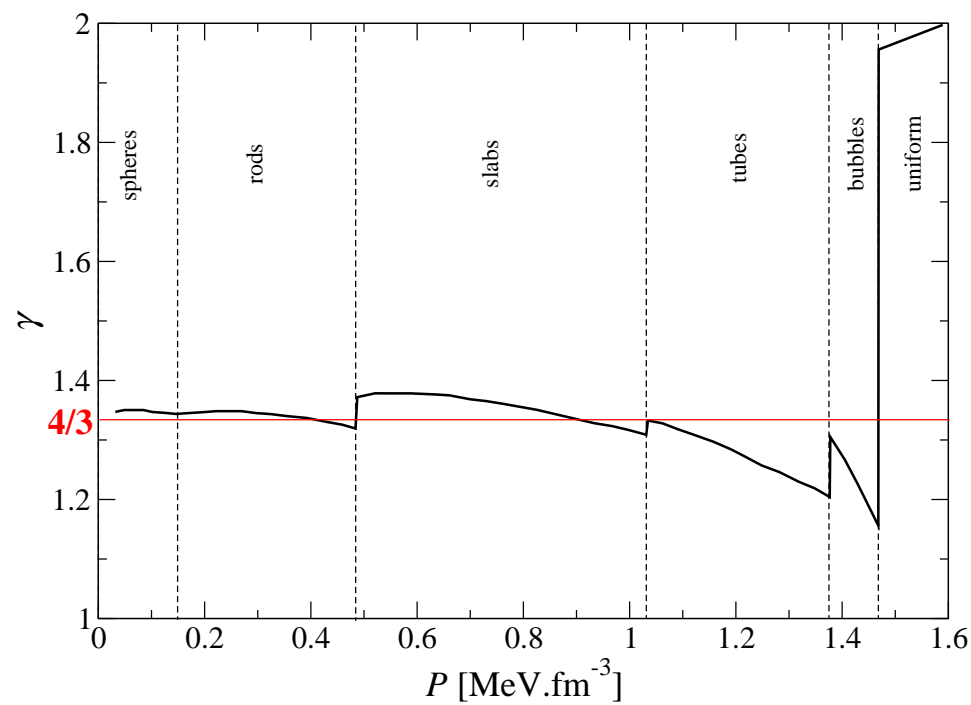

Figure 36: Variation of the adiabatic index of supernova matter, $\gamma$, with pressure $P$ in the nuclear pasta phases for a fixed electron fraction $Y_{e}=0.285$ and the entropy per nucleon $1 k_{\mathrm{B}}$, from the Thomas-Fermi calculations with the Skyrme interaction SkM of Lassaut et al. [252]. 


\section{Crust in Global Neutron Star Structure}

The equilibrium structure of the crust of a neutron star results from the balance of pressure, electromagnetic and elastic stresses, and gravitational pull exerted by the whole star. The global structure of the crust can be calculated by solving Einstein's equations

$$
R_{\mu \nu}-\frac{1}{2} R g_{\mu \nu}=\frac{8 \pi G}{c^{4}} T_{\mu \nu}
$$

where $G$ is the gravitational constant and $c$ the speed of light. The Ricci scalar $R_{\mu \nu}$ and the scalar curvature $R$ are determined from the spacetime metric $g_{\mu \nu}$, which represents gravity. Pressure, electromagnetic and elastic stresses are taken into account in the energy-momentum tensor $T_{\mu \nu}$.

As a first approximation, treating the star as an ideal fluid, the energy-momentum tensor is given by (see, e.g., Landau \& Lifshitz [248])

$$
T_{\nu}^{\mu(\text { liq })}=\left(\rho c^{2}+P\right) u^{\mu} u_{\nu}+P \delta_{\nu}^{\mu}
$$

where $\rho$ is the mass-energy density and $u^{\mu}$ is the 4 -velocity of the fluid. Equation (84) can be written in an equivalent but more general form [70]

$$
T_{\nu}^{\mu(\mathrm{liq})}=n^{\mu} \pi_{\nu}+P \delta_{\nu}^{\mu}
$$

where $n^{\mu}=n u^{\mu}$ is the total 4-current, $n$ is the total particle number density in the fluid rest frame and $\pi_{\nu}$ is the momentum per particle of the fluid given by

$$
\pi_{\nu}=\mu u_{\nu}
$$

The quantity $\mu$ is a dynamic effective mass defined by the relation

$$
P=(n \mu-\rho) c^{2}
$$

where $c$ is the speed of light. As shown by Carter \& Langlois [80], Equation (85) can be easily transposed to fluid mixtures (in order to account for superfluidity inside the star) as follows

$$
T_{\nu}^{\mu(\operatorname{mix})}=\sum n_{\mathrm{x}}^{\mu} \pi_{\nu}^{\mathrm{x}}+\Psi \delta_{\nu}^{\mu}
$$

where X labels matter constituents and $\Psi$ is a generalized pressure, which is not simply given by the sum of the partial pressures of the various constituents (see Section 10).

The electromagnetic field can be taken into account by including the following contribution to the stress-energy tensor

$$
T_{\nu}^{\mu(\mathrm{em})}=\frac{1}{4 \pi} F^{\mu \rho} F_{\nu \rho}-\delta_{\nu}^{\mu} \frac{1}{8 \pi} F^{\sigma \rho} F_{\sigma \rho}
$$

where $F_{\mu \nu}$ is the electromagnetic 2-form. Likewise elastic strains in the solid crust contribute through an additional term $T_{\nu}^{\mu}$ (elast). While the components of $T_{\nu}^{\mu \text { (elast) }}$ are small compared to $P$ (remember that the shear modulus is $\sim 10^{-2}$ of $P$, Section 7 ), it can produce nonaxial deformations in rotating neutron stars, and nonsphericity in nonrotating stars, as discussed in Section 12.5. For the time being, we will use the ideal-fluid approximation, $T_{\nu}^{\mu} \approx T_{\nu}^{\mu(\mathrm{liq})}+T_{\nu}^{\mu}(\mathrm{em})$, and consider the effect of $T_{\nu}^{\mu}$ (elast) on neutron star structure in Section 12.5. We will, therefore, consider purelyhydrostatic equilibrium of the crust, instead of a more general hydro-elastic equilibrium. 


\subsection{Spherical nonrotating neutron stars}

A static (nonrotating) neutron star, built of a perfect fluid, has spherical symmetry. The spacetime is also spherically symmetric, with the metric (see, for instance, Landau \& Lifshitz [248]; here we use the notation of Haensel, Potekhin and Yakovlev [184])

$$
\mathrm{d} s^{2}=c^{2} \mathrm{~d} t^{2} \mathrm{e}^{2 \Phi}-\mathrm{e}^{2 \lambda} \mathrm{d} r^{2}-r^{2}\left(\mathrm{~d} \theta^{2}+\sin ^{2} \theta \mathrm{d} \phi^{2}\right),
$$

where $t$ is a time coordinate, $r$ is a radial coordinate called "circumferential radius" (see below), and $\theta$ and $\phi$ are polar and azimuthal angular coordinates. The dimensionless metric function $\Phi=\Phi(r)$ and $\lambda=\lambda(r)$ have to be determined from Einstein's equations. For a flat (Minkowski) spacetime we would have $\Phi=\lambda=0$. One can show that $\lambda(r)$ is determined by the mass-energy contained within radius $r$, divided by $c^{2}$, to be denoted by $m(r)$ [248],

$$
\mathrm{e}^{-\lambda(r)}=\left(1-\frac{2 G m(r)}{r c^{2}}\right)^{1 / 2}
$$

Let us limit ourselves to the static case $t=$ const. Fixing $r, \theta=\pi / 2$, and then integrating $\mathrm{d} s$ over $\phi$ from zero to $2 \pi$, we find that the proper length of the equator of the star, i.e., its circumference, as measured by a local observer, is equal to $2 \pi r$. This is why $r$ is called the circumferential radius. Notice that Equation (90) implies that the infinitesimal proper radial distance (corresponding to the infinitesimal difference of radial coordinates $\mathrm{d} r$ ) is given by $\mathrm{d} \ell=$ $\mathrm{e}^{\lambda} \mathrm{d} r$.

From Einstein's equations, we get the (relativistic) equations of hydrostatic equilibrium for a static spherically-symmetric star

$$
\begin{gathered}
\frac{\mathrm{d} P}{\mathrm{~d} r}=-\frac{G \rho m}{r^{2}}\left(1+\frac{P}{\rho c^{2}}\right)\left(1+\frac{4 \pi P r^{3}}{m c^{2}}\right)\left(1-\frac{2 G m}{c^{2} r}\right)^{-1} \\
\frac{\mathrm{d} m}{\mathrm{~d} r}=4 \pi r^{2} \rho \\
\frac{\mathrm{d} \Phi}{\mathrm{d} r}=-\frac{1}{\rho c^{2}} \frac{\mathrm{d} P}{\mathrm{~d} r}\left(1+\frac{P}{\rho c^{2}}\right)^{-1} .
\end{gathered}
$$

Equation (92) is the famous Tolman-Oppenheimer-Volkoff equation of hydrostatic equilibrium [407, 313]. Equation (93) enables one to calculate $m(r)$ within a radius $r$. Finally, Equation (94) determines the metric function $\Phi(r)$. Equations (92)-(94) have to be supplemented with an equation of state $(\mathrm{EoS}) P=P(\rho)$.

Let us consider the differential Equations (92) and (93), which determine the global structure of a neutron star. They are integrated from the star center, $r=0$, with the boundary conditions $\rho(0)=\rho_{\mathrm{c}}\left[P(0)=P\left(\rho_{\mathrm{c}}\right)\right]$ and $m(0)=0$. It is clear from Equation (92), that pressure is strictly decreasing with increasing $r$. The integration is continued until $P=0$, which corresponds to the surface of the star, with radial coordinate $r=R$, usually called the star radius.

The gravitational mass of the star is defined by $M=m(R)$. The mass $M$ is the source of the gravitational field outside the star $(r>R)$, and creates an outer spacetime described by the Schwarzschild metric,

$$
r>R: \quad \mathrm{d} s^{2}=c^{2} \mathrm{~d} t^{2}\left(1-\frac{2 G M}{r c^{2}}\right)-\mathrm{d} r^{2}\left(1-\frac{2 G M}{r c^{2}}\right)^{-1 / 2}-r^{2}\left(\mathrm{~d} \theta^{2}+\sin ^{2} \theta \mathrm{d} \phi^{2}\right) .
$$

The crust corresponds to the layer $r_{\mathrm{cc}}<r<R$, where $r_{\mathrm{cc}}$ determines the crust-core interface. The depth below the stellar surface, $z$, is defined as the proper radial distance between the star 
surface and a given surface of radius $r$. It is given by

$$
z(r)=\int_{r}^{R} \mathrm{e}^{\lambda} \mathrm{d} r .
$$

The structure of the crust depends on its EoS, stellar mass, and the EoS of its liquid core. In Figures 37 and 38 we present the structure of the crust of a $1.4 M_{\odot}$ star, for two EoSs of the neutron star interior.

An accreted crust has a different composition and thus a different EoS (stiffer) than the groundstate crust (see Sections 4 and 5). For the comparison to be meaningful, however, these two EoSs have to be calculated from the same nuclear Hamiltonian. We satisfied this by using in both cases the same compressible liquid drop model of Mackie \& Baym [277]. The plots of $\Delta M(z)$ for the ground state and accreted crust of a $1.4 M_{\odot}$ neutron star are shown in Figure 39.

\subsection{Approximate formulae}

For astrophysically relevant neutron star masses $M>M_{\odot}$, the gravitational mass of the crust $M_{\mathrm{cr}}=M-m\left(r_{\mathrm{cc}}\right)$, where $r_{\mathrm{cc}}$ is the radial coordinate of the crust-core interface, constitutes less than $3 \%$ of $M$. Moreover, for realistic EoSs and $M>M_{\odot}$, the difference $\Delta R=R-r_{\mathrm{cc}}$ does not exceed $15 \%$ of $R$. Clearly, $M_{\mathrm{cr}} / M \ll 1$ and an approximation in which the terms $\mathcal{O}\left(M_{\mathrm{cr}} / M\right)$ are neglected is usually sufficiently precise. Neglecting the terms $\mathcal{O}(\Delta R / R)$ gives a less accurate but still useful approximation. In what follows we will use the above "light and thin crust approximation" to obtain useful approximate expressions for the crustal parameters.

Let us first derive an approximate equation for hydrostatic equilibrium within the crust. Let $z$ be the proper depth below the neutron star surface, Equation (96). Within the crust, one can approximate $z$ by

$$
z \approx \frac{R-r}{\sqrt{1-r_{\mathrm{g}} / R}}
$$

where $r_{\mathrm{g}}=2 G M / c^{2}$ is the Schwarzschild radius of mass $M$.

In Equation (92) one can use the approximation $m \simeq M$, and neglect $P / \rho c^{2}$ and $4 \pi P r^{3} / M c^{2}$ as compared to one. Then the equation for hydrostatic equilibrium can be rewritten in a Newtonian form,

$$
\frac{\mathrm{d} P}{\mathrm{~d} z}=g_{\mathrm{s}} \rho
$$

where $g_{\mathrm{s}}$ is the surface gravity,

$$
g_{\mathrm{s}}=\frac{G M}{R^{2} \sqrt{1-r_{\mathrm{g}} / R}} .
$$

Using $P(z=0)=0$, we obtain a formula relating the pressure at depth $z$ to the mass $\Delta M(z)$ of the crust layer above $z$,

$$
\Delta M(z)=4 \pi \frac{R^{2}}{g_{\mathrm{s}}} \sqrt{1-\frac{r_{\mathrm{g}}}{R}} P(z) .
$$

In particular, putting $z=z_{\mathrm{cc}}$, and denoting the pressure at the crust-core interface by $P_{\mathrm{cc}}$, we get

$$
M_{\mathrm{cr}} \approx \frac{4 \pi R^{2} P_{\mathrm{cc}}}{G M}\left(1-\frac{r_{\mathrm{g}}}{R}\right) .
$$

Consider a slow rigid rotation, meaning that the angular frequency of rotation, $\Omega$, as measured by an observer at infinity, is small compared to the mass shedding frequency, $\Omega_{\mathrm{ms}}$. It can be 

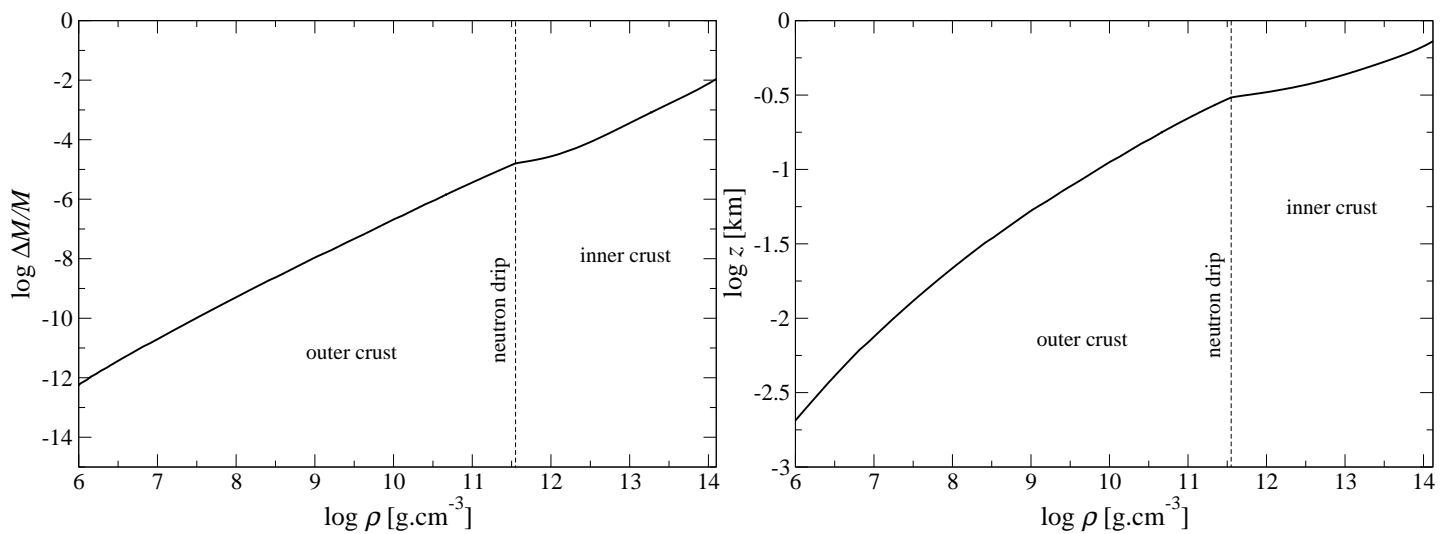

Figure 37: Mass of the crust for the SLy EoS [125]. The neutron star mass is $M=1.4 M_{\odot}$. For this EoS, spherical nuclei persist down to the crust-core interface. Left panel: fractional mass of the crust shell, $\Delta M / M$, vs. its bottom density $\rho$. Right panel: proper depth below the star surface, $z$ vs. mass density $\rho$.
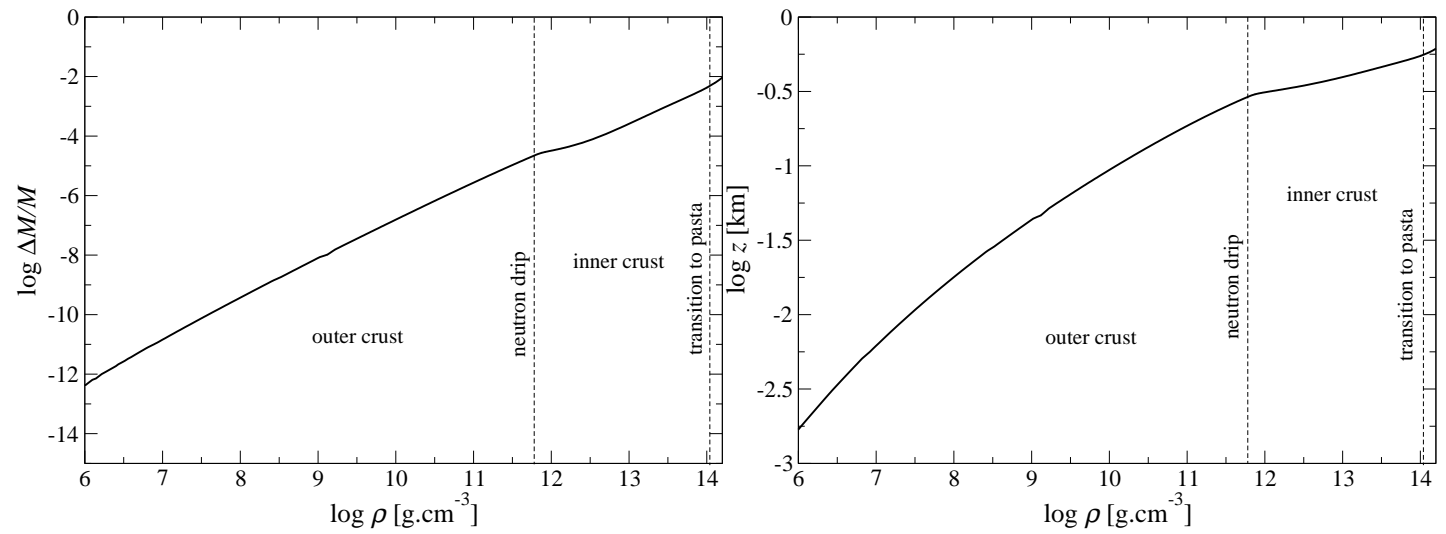

Figure 38: Mass of the crust for the FPS EoS [274]. The neutron star mass is $M=1.4 M_{\odot}$. Notice the presence of the pasta layer, which are absent for the SLy EoS. The pasta phases occupy a thin density layer, but contain about $48 \%$ of the crust mass. Left panel: fractional mass of the crust shell, $\Delta M / M$, vs. its bottom density $\rho$. Right panel: proper depth below the star surface, $z$ vs. mass density $\rho$. 

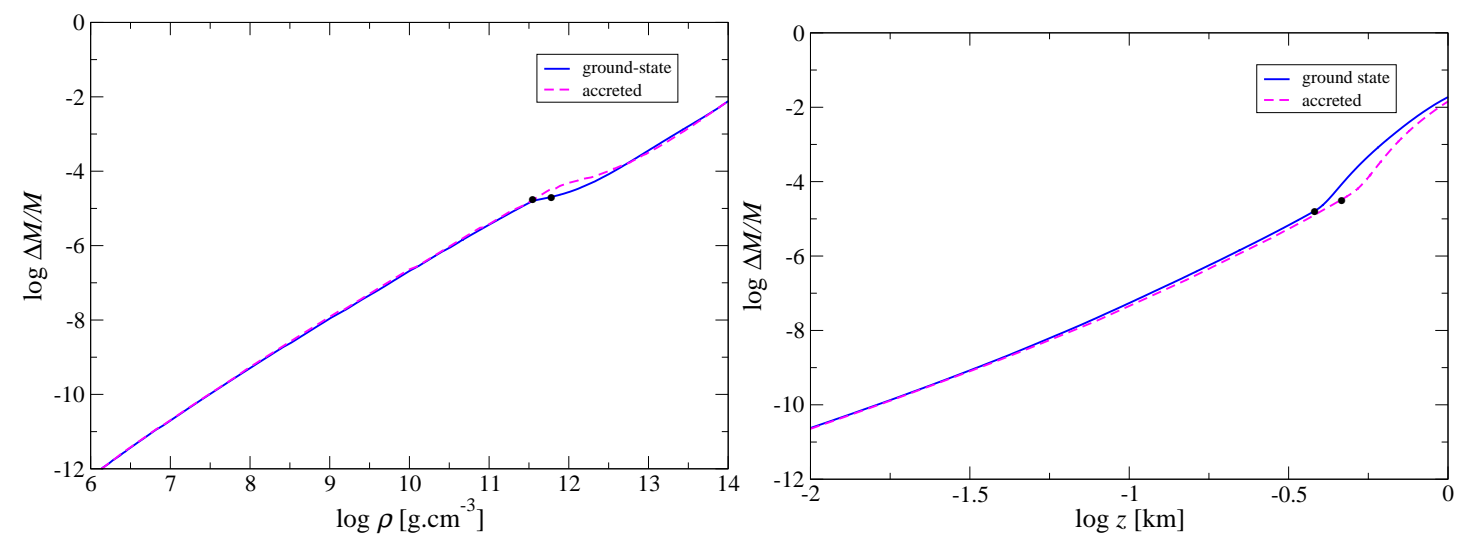

Figure 39: Mass of the crust shell for the ground-state crust and for the accreted crust. The total stellar mass is $M=1.4 M_{\odot}$. Accreted crust: EoS of Haensel \& Zdunik [185]. Ground-state crust: same compressible liquid drop model of atomic nuclei of Mackie \& Baym [277], but full thermodynamic equilibrium (cold catalyzed matter). The black dots indicate the neutron drip transition. Left panel: fractional mass of the crust shell, $\Delta M / M$, vs. its bottom density $\rho$. Right panel: $\Delta M / M$ versus depth below the star surface, $z$.

shown that for slow rigid rotation the moment of inertia, $I$, then involves only the structure of the nonrotating neutron star [190] (see also [166, 184]). Using our notation,

$$
I=\frac{8 \pi}{3} \int_{0}^{R} \mathrm{~d} r r^{4}\left(\rho+\frac{P}{c^{2}}\right) \frac{\bar{\omega}}{\Omega} \mathrm{e}^{-\lambda-\Phi},
$$

where $\bar{\omega}$ is the local rotation frequency, as measured in the local inertial frame. It can be calculated from an ordinary differential equation derived by Hartle [190], with boundary conditions,

$$
\bar{\omega}(R)=\Omega-\frac{2 G J}{R^{3} c^{2}},
$$

where $J$ is the stellar angular momentum.

The contribution of the crust comes from the spherical outer shell, $r_{\mathrm{cc}}<r<R$,

$$
I_{\mathrm{cr}}=\frac{8 \pi}{3} \int_{r_{\mathrm{cc}}}^{R} \mathrm{~d} r r^{4} \rho \frac{\bar{\omega}}{\Omega} \mathrm{e}^{-\lambda-\Phi},
$$

where $P / \rho c^{2}$ was neglected compared to one. Approximating the integrand by its value at $r=R$, and using $\mathrm{e}^{\Phi(R)}=\mathrm{e}^{-\lambda(R)}=\left(1-r_{\mathrm{g}} / R\right)^{1 / 2}$ and Equations (103) and (101), we get

$$
I_{\mathrm{cr}}=\frac{2}{3} M_{\mathrm{cr}} R^{2}\left(1-\frac{r_{\mathrm{g}}}{R} \frac{I}{M R^{2}}\right) .
$$

\subsection{Crust in rotating neutron stars}

We consider stationary neutron-star rotation and assume, for the time being, perfect axial symmetry. Consequently, the spacetime metric is axially symmetric too. Using coordinates $t, r, \theta$, and $\phi$, we can write the spacetime metric in the form (we use the notation of Haensel, Potekhin and Yakovlev [184])

$$
\mathrm{d} s^{2}=c^{2} \mathrm{~d} t^{2} \mathrm{e}^{2 \Phi}-\mathrm{e}^{2 \lambda} r^{2} \sin ^{2} \theta(\mathrm{d} \phi-\omega \mathrm{d} t)^{2}-\mathrm{e}^{2 \alpha}\left(\mathrm{d} r^{2}+r^{2} \mathrm{~d} \theta^{2}\right),
$$


where the metric functions $\Phi, \lambda, \omega$, and $\alpha$ depend on $r$ and $\theta$. The metric function $\omega(r, \theta)$ has the asymptotic behavior $\omega(r \longrightarrow \infty)=0$. It is usually referred to as the "angular velocity of the local inertial frames". Einstein's equations for a stationary rigid rotation of perfect fluid stars can be reduced to a set of $2-\mathrm{D}$ coupled partial differential equations [53, 389]. Their numerical solution can now be obtained using publicly available domain codes, for example, the code rotstar from the LORENE library [270] and the code RNS [388].

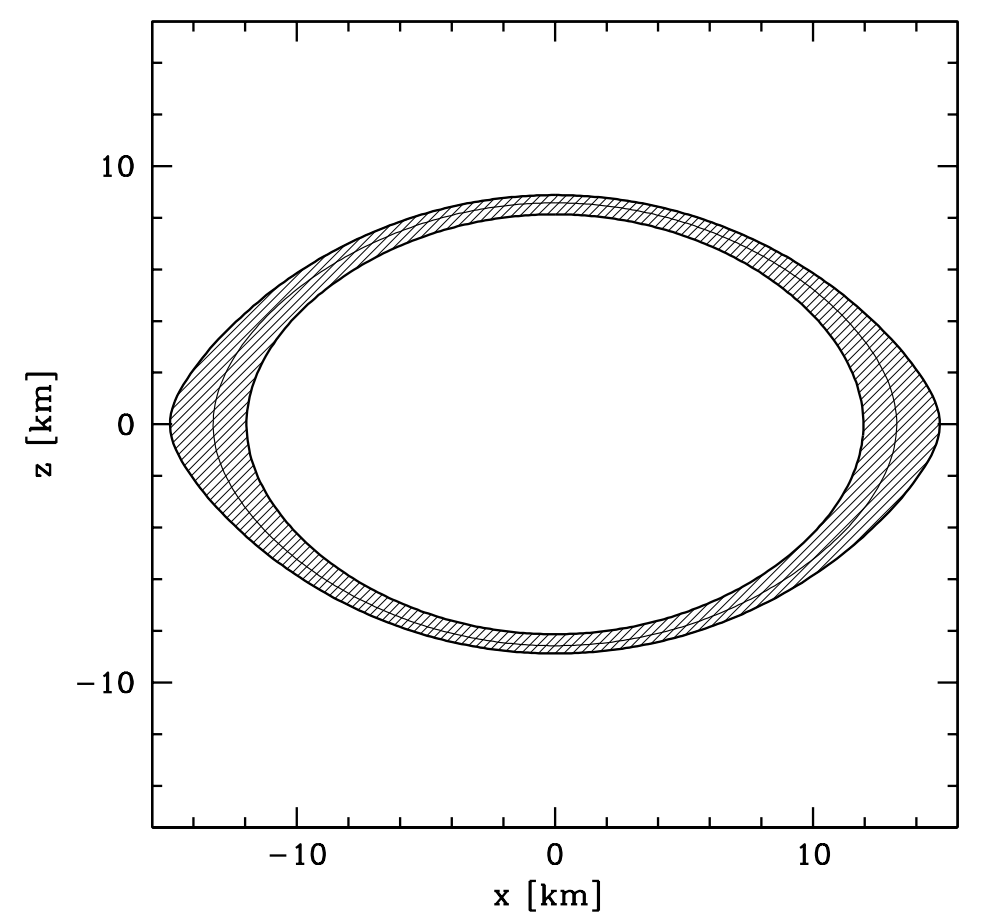

Figure 40: Cross section in the plane passing through the rotation axis of a neutron star of $M=1.82 M_{\odot}$, rotating at $1200 \mathrm{~Hz}$. The SLy EoS for crust and core [125] is used. The coordinates are defined by: $x=r \sin \theta \cos \phi$ and $z=r \cos \theta$, while $r, \theta$, and $\phi$ are metric coordinates, Equation (106). The contours are lines of constant density. Inner contour: crust-core interface. Intermediate contour: outer-inner crust interface. Outer contour: stellar surface. Figure made by J.L. Zdunik.

As can be seen on Figure 40, the outer crust is the most strongly deformed by the centrifugal force. The spin frequency of $1200 \mathrm{~Hz}$, significantly larger than the highest detected up to now, $716 \mathrm{~Hz}$, was chosen to make the effect more spectacular. Let us nevertheless point out that some evidence suggesting the existence of a rapidly-rotating neutron star has recently been found in XTE J1739-285 [227] with a spin frequency of about $1122 \mathrm{~Hz}$. Let us consider, for example, the difference between the equatorial (maximal) and polar (minimal) thickness of the crust, $\Delta R_{\text {eq-pol }}=$ $\Delta R_{\mathrm{eq}}-\Delta R_{\mathrm{pol}}$. For a $\Omega$, which is not too close to the mass-shedding limit, $\Delta R_{\mathrm{eq}-\mathrm{pol}} \propto \Omega^{2}$. Therefore, $\left.\left.\Delta R_{\text {eq-pol }}\right|_{716 \mathrm{~Hz}} \approx 0.4 \Delta R_{\text {eq-pol }}\right|_{1200 \mathrm{~Hz}}$.

The crustal baryon mass (not to be confused with the gravitational mass) $M_{\mathrm{b}, \mathrm{cr}}(\Omega)$ of a neutron star rotating at angular frequency $\Omega$, is larger than the crustal baryon mass $M_{\mathrm{b}, \mathrm{cr}}(0)$ of the static star (with the same total baryon mass). The baryon mass (also called the rest mass) of a star is equal to the number $A$ of baryons it contains times an assumed baryon mass $m_{\mathrm{b}}$. One may take $m_{\mathrm{b}}=m_{n}$ or $m_{\mathrm{b}}=m_{\mathrm{u}}$. We take $M_{\mathrm{b}, \mathrm{cr}}=A m_{\mathrm{u}}$. For $\Omega$ not too close to $\Omega_{\mathrm{ms}}$, we have 


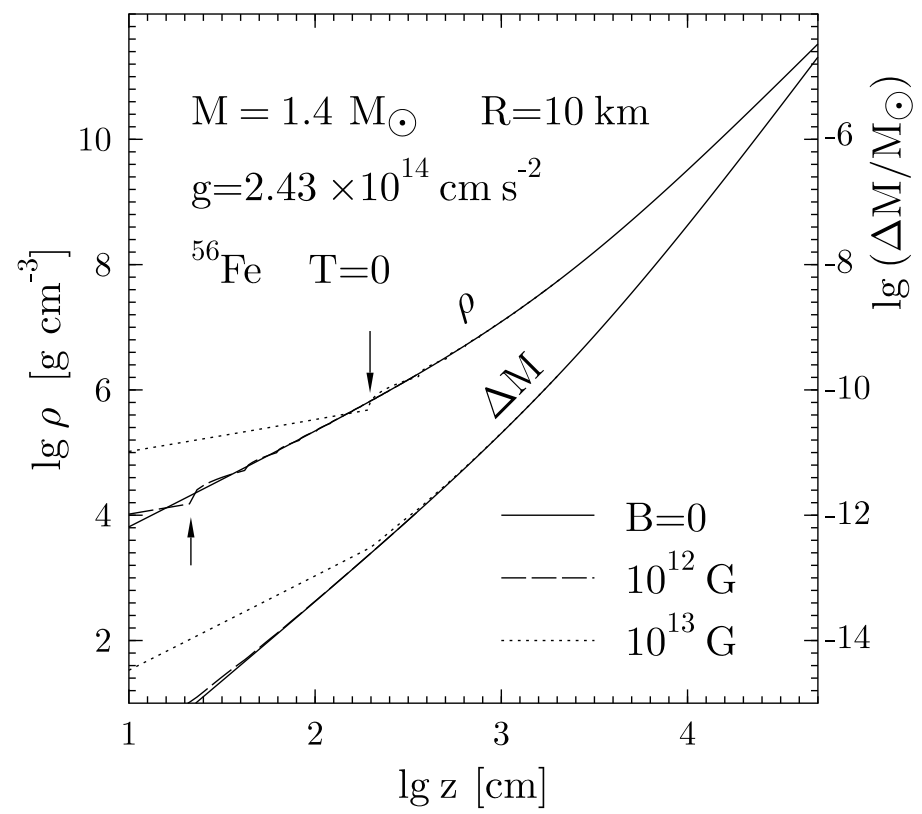

Figure 41: Nonmagnetized and magnetized pure ${ }^{56} \mathrm{Fe}$ crust in a neutron star with $M=1.4 M_{\odot}$ and $R=10 \mathrm{~km}$ and at $T=0$. Matter density (left vertical scale) and the mass of the outer shell $\Delta M$ (right vertical axis) versus depth below the surface $z$, for $B=0, B=10^{12} \mathrm{G}$, and $B=10^{13} \mathrm{G}$. Arrows indicate densities (kinks) at which the $n=1$ Landau level starts to be populated with increasing depth. Figure 6.15 from [184]. 
$M_{\mathrm{b}, \mathrm{cr}}(\Omega)-M_{\mathrm{b}, \mathrm{cr}}(0) \propto \Omega^{2}$. Due to the radiation of electromagnetic waves and particles, a pulsar spins down, so that $\dot{\Omega}<0$. Consequently, the baryon mass of the pulsar crust decreases in time, $\dot{M}_{\text {b,cr }} \propto \dot{\Omega} \Omega<0$. Nucleons pass from the crust to the liquid core, releasing some heat. As shown in Figure 40, the crust is decompressed near the equator and compressed near the pole. These deformations trigger various nuclear reactions involving electrons, neutrons, and nuclei. These reactions tend to drive the deformed crust towards its equilibrium shape and release heat, which influences the cooling of a spinning down pulsar [205]. Additional heating results from crust cracking when local shear strain exceeds the maximal one.

\subsection{Effects of magnetic fields on the crust structure}

The structure of the outer layers of the crust (neutron star "envelope") can be affected by the presence of a magnetic field. Effects of magnetic fields on the EoS were briefly mentioned in Section 2.2. Here we consider examples showing effects of $\boldsymbol{B}$ on the crust structure.

Typical values of the surface magnetic fields of radio pulsars are $B \sim 10^{12} \mathrm{G}$. In Figure 41 we compare plots of $\rho(z)$ and $\Delta M(z)$ for the pure iron envelope with $\rho \lesssim 10^{5} \mathrm{~g} \mathrm{~cm}^{-3}$ of a neutron star with $M=1.4 M_{\odot}$ and $R=10 \mathrm{~km}$ with and without a magnetic field. The $T=0$ approximation for the crust is used. Typical values of the surface magnetic field of radio pulsars are $B \sim 10^{12} \mathrm{G}$. At such magnetic fields, the effect of $B$ is seen only in the outer envelope, which is $\sim 30 \mathrm{~cm}$ thick. We can see there quantum oscillations of the density as a function of depth. They are associated with the filling of the lowest Landau levels $n=0,1, \ldots$ by the electrons (Section 2.2). Increasing $B$ to $10^{13} \mathrm{G}$, associated with the most magnetized radio pulsars, leads to much deeper magnetization of the crust, down to the depth of $30 \mathrm{~m}$, where the prevailing density reaches $10^{6} \mathrm{~g} \mathrm{~cm}^{-3}$. The effect of the magnetic field in the outer $30 \mathrm{~cm}$ of the crust is dramatic; in spite of a gravitational acceleration $g=G M / R^{2}=2.43 \times 10^{14} \mathrm{~cm} \mathrm{~s}^{-2}$, the density is only slowly decreasing, and is still $\sim 10^{5} \mathrm{~g} \mathrm{~cm}^{-3}$ at $10 \mathrm{~cm}$ depth, ten times higher than in a nonmagnetized envelope at the same depth. So, magnetized iron plasma is "condensed", and much less compressible than nonmagnetized (Section 2.2). At $10^{13} \mathrm{G}$, the $n=1$ Landau level begins to be populated only at $4 \times 10^{5} \mathrm{~g} \mathrm{~cm}^{-3}$ (depth $3 \mathrm{~m}$ ), to be compared with $2 \times 10^{4} \mathrm{~g} \mathrm{~cm}^{-3}($ depth $30 \mathrm{~cm})$ at $10^{12} \mathrm{G}$. It should be stressed that, as the surface temperature at pulsar age $10^{3}-10^{4}$ y is $T_{\mathrm{s}} \sim 10^{6} \mathrm{~K}$, inclusion of $T$ will weaken the magnetization effects on the structure of the crust [184].

Typical surface magnetic fields of magnetars are $\sim 10^{15}$ G. In Figure 42 we see that for $\rho<$ $10^{9} \mathrm{~g} \mathrm{~cm}^{-3}$, the effect of such a huge $\boldsymbol{B}$ on the crust EoS is strong and becomes dramatic at lower $\rho$. For example, at $10^{7} \mathrm{~g} \mathrm{~cm}^{-3}$, the matter pressure decreases by two orders of magnitude, compared to the $\boldsymbol{B}=0$ case. The $n=1$ Landau level begins to be populated only at $10^{8.7} \mathrm{~g} \mathrm{~cm}^{-3}$, at a depth of about $50 \mathrm{~m}$. 

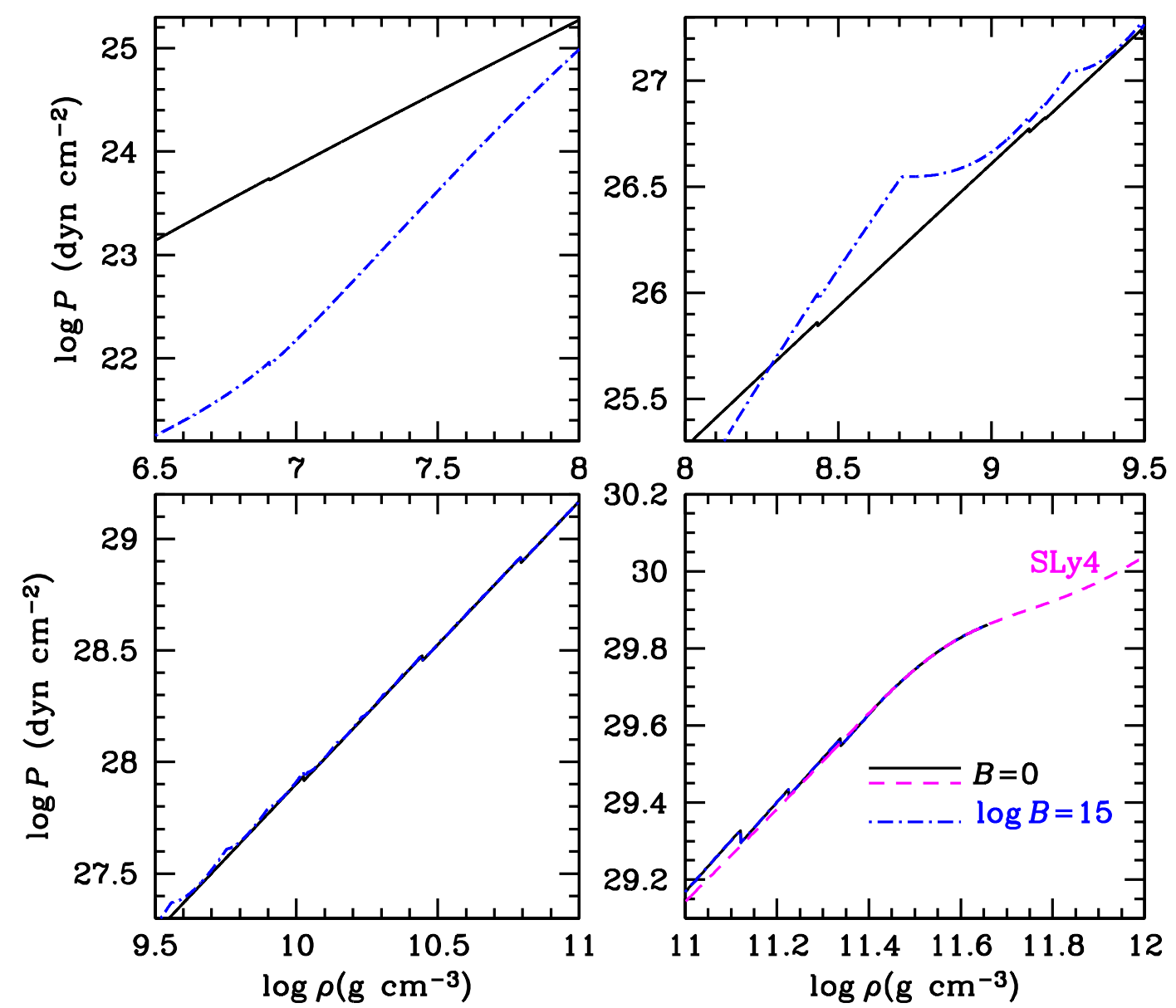

Figure 42: Nonmagnetized (solid line), and magnetized crust (dash-dotted line) calculated with ground state composition calculated at $\boldsymbol{B}=0$. The dashed magenta line in the bottom-right panel corresponds to the $\boldsymbol{B}=0$ compressible liquid drop model of [125], and is smooth due to its quasiclassical nature, while the curve with discontinuous drops in pressure as obtained using the ground-state model of [183]. Figure made by A.Y. Potekhin. 


\section{$7 \quad$ Elastic Properties}

A solid crust can sustain an elastic strain up to a critical level, the breaking strain. Neutron stars are relativistic objects, and therefore a relativistic theory of elastic media in a curved spacetime should be used to describe elastic effects in neutron star structures and dynamics. Such a theory of elasticity has been developed by Carter \& Quintana [82], who applied it to rotating neutron stars in [83, 84] (see also Beig [44] and references therein). Recently, Carter and collaborators have extended this theory to include the effects of the magnetic field [73], as well as the presence of the neutron superfluid, which permeates the inner crust $[72,85]$. For the time being, for the sake of simplicity, we ignore magnetic fields and free neutrons. However, in Section 7.2 the effect of free neutrons on the elastic moduli of the pasta phases is included, within the compressible liquid drop model. Since relativistic effects are not very large in the crust, we shall restrict ourselves to the Newtonian approximation (see, e.g., [249]).

The thermodynamic equilibrium of an element of neutron-star crust corresponds to equilibrium positions of nuclei, which will be denoted by a set of vectors $\{\boldsymbol{r}\}$, which are associated with the lattice sites. Neutron star evolution, driven by spin-down, accretion of matter or some external forces, like tidal forces produced by a close massive body, or internal electromagnetic strains associated with strong magnetic fields, may lead to deformation of this crust element as compared to the equilibrium state.

For simplicity, we will neglect thermal contributions to thermodynamic quantities and restrict ourselves to the $T=0$ approximation. Deformation of a crust element with respect to the equilibrium configuration implies a displacement of nuclei into their new positions $\boldsymbol{r}^{\prime}=\boldsymbol{r}+\boldsymbol{u}$, where $\boldsymbol{u}=\boldsymbol{u}(\boldsymbol{r})$ is the displacement vector. In the continuum limit, valid for macroscopic phenomena, both $\boldsymbol{r}$ and $\boldsymbol{u}$ are treated as continuous fields. Nonzero $\boldsymbol{u}$ is associated with elastic strain (i.e., forces which tend to return the matter element to the equilibrium state of minimum energy density $\varepsilon_{0}$ ), and with the deformation energy density $\varepsilon_{\text {def }}=\varepsilon(\boldsymbol{u})-\varepsilon_{0}{ }^{4}$.

A uniform translation does not contribute to $\varepsilon_{\text {def }}$, and the true deformation is described by the (symmetric) strain tensor

$$
\mathrm{u}_{i k}=\mathrm{u}_{k i}=\frac{1}{2}\left(\frac{\partial u_{i}}{\partial x_{k}}+\frac{\partial u_{k}}{\partial x_{i}}\right),
$$

where $i, j=1,2,3$, and $x_{1}=x, x_{2}=y, x_{3}=z$. This formula for $\mathrm{u}_{i k}$ is valid if the displacement vector $\boldsymbol{u}$ is small, so that the terms quadratic in the components of $\mathbf{u}$ can be neglected compared to the linear ones [249].

Any deformation can be decomposed into compression and shear parts,

$$
\mathrm{u}_{i k}=\mathrm{u}_{i k}^{\text {comp }}+\mathrm{u}_{i k}^{\text {shear }}, \text { where } \quad \mathrm{u}_{i k}^{\text {comp }}=\frac{1}{3}(\boldsymbol{\nabla} \cdot \boldsymbol{u}) \delta_{i k}, \mathrm{u}_{i k}^{\text {shear }}=\mathrm{u}_{i k}-\frac{1}{3}(\boldsymbol{\nabla} \cdot \boldsymbol{u}) \delta_{i k} .
$$

Under deformation, matter element volume changes according to $\mathrm{d} V^{\prime}=[1+(\nabla \cdot \boldsymbol{u})] \mathrm{d} V$. A pure compression (no shear) of the matter element is described by $u_{i k}=\alpha \delta_{i k}$. For volume preserving deformations $(\boldsymbol{\nabla} \cdot \boldsymbol{u})=0$.

To lowest order, the deformation energy is quadratic in the deformation tensor,

$$
\varepsilon_{\mathrm{def}}=\sum_{i k l m} \frac{1}{2} \lambda_{i k l m} \mathrm{u}_{i k} \mathrm{u}_{l m} .
$$

Since $\varepsilon_{\text {def }}$ is a scalar, $\lambda_{i k l m}$ is a fourth rank tensor. The total number of $\lambda_{i k l m}$ components is eighty one; general symmetry relations reduce the maximum number of linearly-independent components (elastic moduli) to twenty one. The number of independent elastic moduli decreases with increasing

\footnotetext{
${ }^{4}$ In this section, by "energy" we will usually mean energy of a unit volume (i.e. energy density).
} 
symmetry of the elastic medium. It is three in the case of a cubic crystal, and two for an isotropic solid (see, e.g., [249]).

The elastic contribution to the stress tensor $\Pi_{i k}^{\text {elast }} \equiv \sigma_{i k}$ is $\sigma_{i k}=\partial \varepsilon_{\text {def }} / \partial u_{i k}$.

\subsection{Isotropic solid (polycrystal)}

Microscopically, the ground state corresponds to a body-centered cubic (bcc) crystal lattice. However, one usually assumes that macroscopically, the neutron star crust is an isotropic bcc polycrystal. Elastic properties of an isotropic solid are described by two elastic moduli. The deformation energy can be expressed as

$$
\varepsilon_{\mathrm{def}}=\frac{1}{2} K(\boldsymbol{\nabla} \cdot \boldsymbol{u})^{2}+\mu\left(u_{i k}-\frac{1}{3} \delta_{i k}(\boldsymbol{\nabla} \cdot \boldsymbol{u})\right)^{2} .
$$

Here, $\mu$ is the shear modulus and $K$ is the compression modulus. The elastic stress tensor is, therefore,

$$
\sigma_{i k}=\frac{\partial \varepsilon_{\mathrm{def}}}{\partial u_{i k}}=K(\boldsymbol{\nabla} \cdot \mathbf{u}) \delta_{i k}+2 \mu\left(u_{i k}-\frac{1}{3}(\boldsymbol{\nabla} \cdot \mathbf{u}) \delta_{i k}\right) .
$$

Considering a small pure uniform compression, one finds

$$
K=n_{\mathrm{b}} \frac{\partial P}{\partial n_{\mathrm{b}}}=\gamma P
$$

where $P$ is the total pressure given by Equation (24) and $\gamma$ is the adiabatic index defined by Equation (80).

Monte Carlo calculations of the effective shear modulus of a polycrystalline bcc Coulomb solid were performed by Ogata \& Ichimaru [309]. The deformation energy, resulting from the application of a specific strain $\mathrm{u}_{i k}$, was evaluated through Monte Carlo sampling.

As we have already mentioned, for an ideal cubic crystal lattice there are only three independent elastic moduli, denoted traditionally as $c_{11}, c_{12}$ and $c_{44}$ (see Chapter 3, pp. 80-87 of the book by Kittel [241]). For pure shear deformation, only two independent elastic moduli are relevant,

$$
\varepsilon_{\mathrm{def}}=b_{11}\left(u_{x x}^{2}+u_{y y}^{2}+u_{z z}^{2}\right)+2 c_{44}\left(u_{x y}^{2}+u_{x z}^{2}+u_{y z}^{2}\right) \quad \text { for } \quad(\nabla \cdot \boldsymbol{u})=0,
$$

because $b_{11}=\frac{1}{2}\left(c_{11}-c_{12}\right)$. At $T=0$, Ogata \& Ichimaru [309] find

$$
\begin{aligned}
& b_{11}=0.0245 n_{\mathrm{N}} \frac{(Z e)^{2}}{R_{\text {cell }}}, \\
& c_{44}=0.1827 n_{\mathrm{N}} \frac{(Z e)^{2}}{R_{\text {cell }}} .
\end{aligned}
$$

These values agree with the classical result of Fuchs [151].

The definition of an "effective" shear modulus of a bcc polycrystal deserves a comment. In numerous papers, a standard preferred choice was $\mu=c_{44}([43,319,285]$, and references therein). However, replacing $\mu$ by a single maximal elastic modulus of a strongly anisotropic bcc lattice is not correct. An effective value of $\mu$ was calculated by Ogata \& Ichimaru [309]. They performed directional averages over rotations of the Cartesian axes. At $T=0$, they obtained

$$
\mu=\frac{1}{5}\left(2 b_{11}+3 c_{44}\right)=0.1194 \frac{n_{\mathrm{N}}(Z e)^{2}}{R_{\text {cell }}},
$$




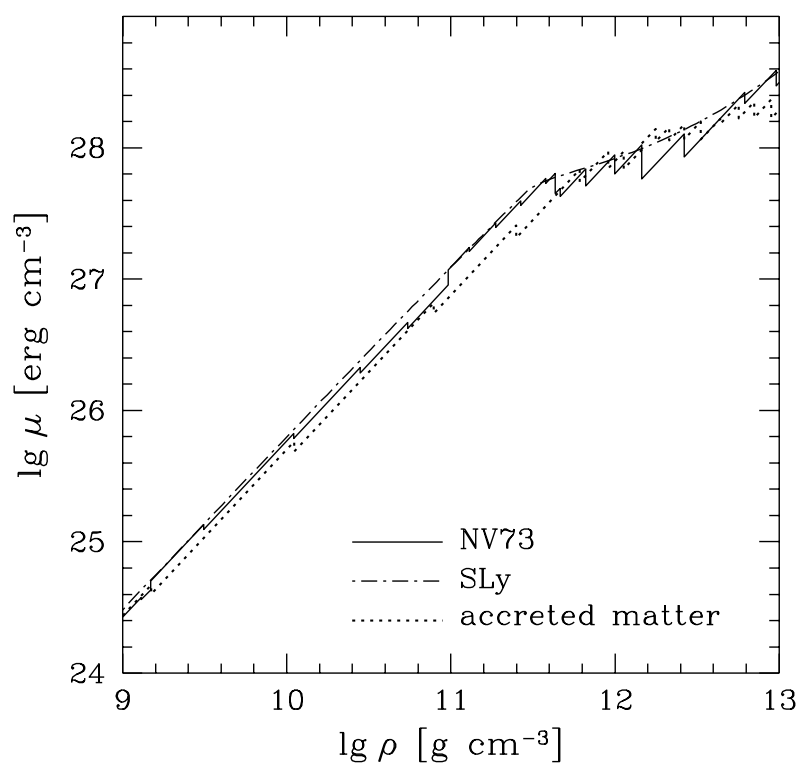

Figure 43: Effective shear modulus $\mu$ versus density, for a bcc lattice. Solid line - cold catalyzed matter (Haensel and Pichon 1994 model [183] for the outer crust (Section 3.1), and that of Negele and Vautherin 1973 [303] for the inner crust (Section 3.2.3)). Dash-dotted line - cold catalyzed matter calculated by Douchin and Haensel 2000 [126] (compressible liquid drop model, based on SLy effective N-N interaction, Section 3.2.1). Dotted line - accreted crust model of Haensel and Zdunik 1990 [185] (Section 4). Figure made by A.Y. Potekhin. 
some $30 \%$ smaller than $\mu=c_{44}$ used in previous papers. Dependence of $\mu$ on temperature was studied, using the Monte Carlo method, by Strohmayer et al. [393]. As expected, the effective shear modulus decreases with increasing temperature.

The formula for $\mu$, Equation (116), can be rewritten as

$$
\mu=0.0159\left(\frac{Z}{26}\right)^{2 / 3} P_{e}
$$

where $P_{e}$ is the pressure of ultrarelativistic degenerate electrons. Therefore,

$$
\frac{\mu}{K}=0.016\left(\frac{Z}{26}\right)^{2 / 3} \frac{P_{e}}{\gamma P} \ll 1 .
$$

The Poisson coefficient $\sigma \simeq 1 / 2$, while the Young modulus $E \simeq 3 \mu$.

Let us remember that the formulae given above hold for the outer crust, where the size of the nuclei is very small compared to the lattice spacing and $P \simeq P_{e}$. For the inner crust these formulae are only approximate.

\section{$7.2 \quad$ Nuclear pasta}

Some theories of dense matter predict the existence of "nuclear pasta" - rods, plates, tubes, bubbles - in the bottom layer of the crust with $\rho \gtrsim 10^{14} \mathrm{~g} \mathrm{~cm}^{-3}$ (see Section 3.3). In what follows we will concentrate on rods (spaghetti) and plates (lasagna). They are expected to fill most of the bottom crust layers. The matter phases containing rods and plates have properties intermediate between solids and liquids. The displacement of an element of matter parallel to the plane containing rods or plates is not opposed by restoring forces: this lack of a shear strain is typical for a liquid. On the contrary, an elastic strain opposes any bending of planes or rods: this is a property of a solid. Being intermediate between solids and liquids, these kinds of matter are usually called mesomorphic phases, or liquid crystals (see, e.g., [249]).

The elastic properties of rod and plate phases of neutron star matter were studied by Pethick \& Potekhin [325]. As they stressed, the physical reasons for the forming of mesomorphic phases in neutron star crusts are very different from those relevant to liquid crystals in laboratory. For terrestrial liquid crystals it is the interaction between very nonspherical molecules, which drives them to form rods and plates. In a neutron star crust the mechanism consists in spontaneous symmetry breaking, resulting from competition between the Coulomb energy and nuclear surface energy. We will follow closely Pethick \& Potekhin [325]. They calculated the energies of mesomorphic phases using the generalized liquid drop model. The plate phase has rotational symmetry about any axis perpendicular to the plates, and is therefore similar to the smectics A phase of liquid crystals [115]. Let the z-axis coincide with the symmetry axis of the equilibrium configuration. Only a displacement in the z-direction is opposed by a restoring force. Therefore, we consider only $\boldsymbol{u}=(0,0, u)$. The deformation energy of a unit volume is then [115]

$$
\varepsilon_{\mathrm{def}}=\frac{1}{2} B\left[\frac{\partial u}{\partial z}-\frac{1}{2}\left(\nabla_{\perp} u\right)^{2}\right]^{2}+\frac{1}{2} K_{1}\left(\nabla_{\perp}^{2} u\right)^{2},
$$

where $\nabla_{\perp} \equiv(\partial / \partial x, \partial / \partial y, 0)$. Using the generalized liquid drop model, Pethick \& Potekhin [325] obtain

$$
B=6 \varepsilon_{\text {Coul }} \quad K_{1}=\frac{2}{15} R_{\text {cell }}^{2} \varepsilon_{\text {Coul }}\left(1+2 w-2 w^{2}\right),
$$

where $\varepsilon_{\text {Coul }}$ is the Coulomb energy density (in equilibrium)

$$
\varepsilon_{\text {Coul }}=\frac{2 \pi}{3}\left(e n_{p \mathrm{i}} R_{\text {cell }}\right)^{2} \frac{(1-w)^{2}}{w},
$$

Living Reviews in Relativity

http: //www. livingreviews . org/Irr-2008-10 
and where $R_{\text {cell }}$ is the half-distance between the plates, $n_{p \mathrm{i}}$ is the proton density in nuclear matter and $w$ is the volume fraction occupied by nuclear matter (all values calculated for the relaxed system).

In the case of the rod phase, also called the columnar phase [115], the number of elastic moduli is larger. They describe the increase in energy density due to compression, dilatation, transverse shearing, and bending of the rod lattice. Elastic moduli were calculated within the liquid drop model by Pethick \& Potekhin [325] and by Watanabe, Iida \& Sato [419, 420].

At the microscopic scale (fermis), the elastic properties of the nuclear pastas are very different from those of a body-centered-cubic crystal of spherical nuclei. Nevertheless, the effects of pasta phases on the elastic properties of neutron star crusts may not be so dramatic at large scales (let's say meters). Indeed these nuclear pastas are necessarily of finite extent since one and twodimensional long-range crystalline orders cannot exist in infinite systems (see, for instance, [157] and references therein). How the nuclear pastas arrange themselves remains to be studied, but it is likely that the resulting configurations look more-or-less isotropic at macroscopic scales. 


\section{Superfluidity and Superconductivity}

Except for a brief period after their birth, neutron stars are expected to contain various superfluid and superconducting phases [363, 116, 29, 367]. In this section, after a brief discussion of superconductivity and its possible occurrence in neutron star crusts, we will review our current theoretical understanding of the static and dynamic properties of neutron superfluid in the inner crust of neutron stars. For a general introduction and a recent overview on superfluidity and superconductivity, see, for instance, the book by Annett [22].

\subsection{Superconductivity in neutron star crusts}

In the Bardeen-Cooper-Schrieffer (BCS) theory of superconductivity [36] the coupling of electrons with lattice vibrations leads to an effective attractive interaction between electrons despite the repulsive Coulomb force. As a result, the electrons of opposite spins form pairs with zero total angular momentum. These Cooper pairs behave like bosons. Unlike fermions, bosons do not obey the Pauli exclusion principle, which forbids multiple occupancy of single particle quantum states. Consequently, below some critical temperature, bosons condense into the lowest-energy single-particle state, giving rise to superfluidity, as in liquid helium-4. Loosely speaking, superconductivity can thus be seen as Bose-Einstein condensation of bound electron pairs. However the analogy with Bose-Einstein condensation should not be taken too far. Indeed, Cooper pairs do not exist above the superconducting transition, while in Bose-Einstein condensation bosons always exist above and below the critical temperature. Besides the electrons in a pair do not form well separated "molecules" like atoms in liquid helium, but instead strongly overlap. The spatial extent of a Cooper pair in a conventional superconductor is typically several orders of magnitude larger than the mean inter-electron spacing. The Bose-Einstein condensation (BEC) and the BCS regime are now understood as two different limits of the same phenomenon as illustrated in Figure 44. The transition between these two limits has recently been studied in ultra-cold atomic Fermi gases, for which the interaction can be adjusted experimentally [103].
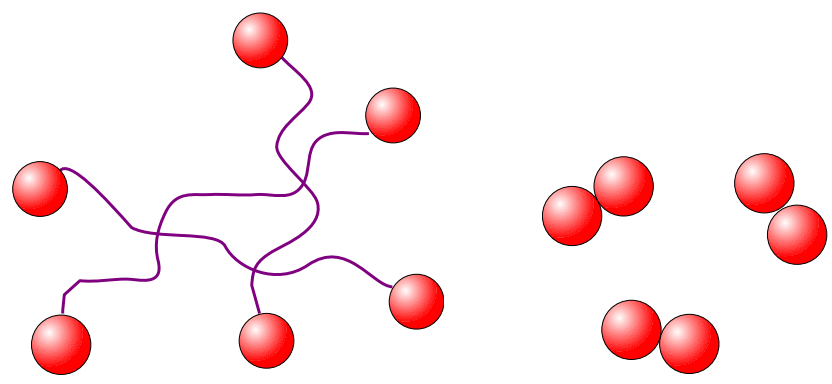

Figure 44: Schematic picture illustrating the difference between the BCS regime (left) of overlapping loosely bound fermion pairs and the BEC regime (right) of strongly bound pairs.

In the outermost envelope of neutron stars, where the density is similar to that of ordinary solids, the critical temperature for the onset of electron superconductivity is, at most, on the order of a few $\mathrm{K}$, which is many orders of magnitude smaller than the expected and observed surface temperature (see Section 12.2). Besides, it is well known that iron, the most probable constituent of the outer layers of the crust (Section 3.1), is not superconducting under normal pressure. It was discovered in 2001 [376] that iron becomes superconducting at "high" pressures 5 between $1.5 \times 10^{11}$ and $3 \times 10^{11} \mathrm{dyn}^{-2}$, when the temperature is below about $2 \mathrm{~K}$. At higher densities, assuming

\footnotetext{
${ }^{5}$ Such pressures are very small in the context of neutron stars. For iron at room temperature they correspond to a density of about $8.2 \mathrm{~g} \mathrm{~cm}^{-3}$, in comparison to $7.86 \mathrm{~g} \mathrm{~cm}^{-3}$ under normal atmospheric pressure [42].
} 
that the electrons are degenerate, we can estimate the critical temperature from the BCS weak coupling approximation [36] (see also the discussion by Ginzburg [160]).

$$
T_{\mathrm{ce}} \sim T_{\mathrm{pi}} \exp \left(-2 / v_{\mathrm{eff}} g_{\mathrm{Fe}}\right),
$$

where $T_{\mathrm{pi}}$ is the ion plasma frequency, $v_{\mathrm{eff}}$ is the effective attractive electron-electron interaction and $g_{\mathrm{Fe}}$ is the density of electron states at the Fermi level (per unit energy and per unit volume). Neglecting band structure effects (which is a very good approximation in dense matter; see, for instance, the discussion of Pethick \& Ravenhall [326]), the density of electron states is given by

$$
g_{\mathrm{Fe}}=\frac{k_{\mathrm{Fe}}^{2}}{\pi^{2} \hbar v_{\mathrm{F} e}},
$$

so that the critical temperature takes the form

$$
T_{\mathrm{ce}} \sim T_{\mathrm{pi}} \exp \left(-\zeta \hbar v_{\mathrm{Fe}} / e^{2}\right),
$$

where $\zeta$ is a numerical positive coefficient of order unity [160]. At densities much below $10^{6} \mathrm{~g} \mathrm{~cm}^{-3}$, the electrons are nonrelativistic and their Fermi velocity is given by $v_{\mathrm{Fe}}=\hbar k_{\mathrm{Fe}} / m_{e}$. As a result, the critical temperature decreases exponentially with the average mass density $\rho$ as

$$
T_{\mathrm{ce}} \sim T_{\mathrm{pi}} \exp \left(-\zeta^{\prime}\left(\rho / \rho_{\mathrm{ord}}\right)^{1 / 3}\right)
$$

where $\zeta^{\prime} \equiv \zeta(9 \pi Z / 4 A)^{1 / 3}$ and $\rho_{\text {ord }}=m_{\mathrm{u}} /\left(4 \pi a_{0}^{3} / 3\right)$ is a typical density of ordinary matter $\left(a_{0}\right.$ is the Bohr radius and $m_{u}$ the atomic mass unit). Considering, for instance, a plasma of iron and electrons, and adopting the value $\zeta=8 / \pi$ calculated for the "jellium" model by Kirzhnits [240], the critical temperature is approximately given by

$$
T_{\mathrm{ce}} \sim 3.6 \times 10^{3}\left(\frac{\rho}{1 \mathrm{~g} \mathrm{~cm}^{-3}}\right)^{1 / 2} \exp \left(-2.7\left(\frac{\rho}{1 \mathrm{~g} \mathrm{~cm}^{-3}}\right)^{1 / 3}\right) \mathrm{K} .
$$

This rough estimate shows that the critical temperature decreases very rapidly with increasing density, dropping from $\sim 30 \mathrm{~K}$ at $\rho=10 \mathrm{~g} \mathrm{~cm}^{-3}$ to $10^{-1} \mathrm{~K}$ at $\rho=10^{2} \mathrm{~g} \mathrm{~cm}^{-3}$ and to $10^{-7} \mathrm{~K}$ at $\rho=10^{3} \mathrm{~g} \mathrm{~cm}^{-3}$ ! At densities above $\sim 10^{6} \mathrm{~g} \mathrm{~cm}^{-3}$, electrons become relativistic, and $v_{\mathrm{Fe}} \sim c$. According to Equation (124), the critical temperature of relativistic electrons is given by

$$
T_{\mathrm{ce}} \sim T_{\mathrm{pi}} \exp (-\zeta / \alpha),
$$

where $\alpha=e^{2} / \hbar c \simeq 1 / 137$ is the fine structure constant. Due to the exponential factor, the critical temperature is virtually zero.

We can, thus, firmly conclude that electrons in neutron star crusts (and, a fortiori, in neutron star cores) are not superconducting. Nevertheless, superconductivity in the crust is not completely ruled out. Indeed, at the crust-core interface some protons could be free in the "pasta" mantle (Section 3.3), and could be superconducting due to pairing via strong nuclear interactions with a critical temperature far higher than that of electron superconductivity. Microscopic calculations in uniform nuclear matter predict transition temperatures on the order of $T_{\mathrm{c} p} \sim 10^{9}-10^{10} \mathrm{~K}$, which are much larger than typical temperatures in mature neutron stars. Some properties of superconductors are discussed in Sections 8.3.3 and 8.3.4.

\subsection{Static properties of neutron superfluidity}

Soon after its formulation, the Bardeen-Cooper-Schrieffer (BCS) theory of electron superconductivity [36] was successfully applied to nuclei by Bohr, Mottelson and Pines [51] and Belyaev [46]. 
In a paper devoted to the moment of inertia of nuclei, Migdal [291] speculated about the possibility that superfluidity could occur in the "neutron core" of stars (an idea which was raised by Gamow and Landau in 1937 as a possible source of stellar energy; see, for instance, [184]). The superfluidity inside neutron stars was first studied by Ginzburg and Kirzhnits in 1964 [161, 162]. Soon after, Wolf [424] showed that the free neutrons in the crust are very likely to be superfluid. It is quite remarkable that the possibility of superfluidity inside neutron stars was raised before the discovery of pulsars by Jocelyn Bell and Anthony Hewish in 1967. Later, this prediction seemed to be confirmed by the observation of the long relaxation time, on the order of months, following the first glitch in the Vela pulsar [41]. The neutron superfluid in the crust is believed to play a key role in the glitch mechanism itself. Pulsar glitches are still considered to be the strongest observational evidence of superfluidity in neutron stars (see Section 12.4).

At the heart of BCS theory is the existence of an attractive interaction needed for pair formation. In conventional superconductors, this pairing interaction is indirect and weak. In the nuclear case the occurrence of superfluidity is a much less subtle phenomenon since the bare strong interaction between nucleons is naturally attractive at not too small distances in many $J L S$ channels ( $J$-total angular momentum, $L$-orbital angular momentum, $S$-spin of nucleon pair). Apart from a proton superconductor similar to conventional electron superconductors, two different kinds of neutron superfluids are expected to be found in the interior of a neutron star (for a review, see, for instance, [363, 271, 116, 29, 367]). In the crust and in the outer core, the neutrons are expected to form an isotropic superfluid like helium-4, while in denser regions they are expected to form a more exotic kind of (anisotropic) superfluid with each member of a pair having parallel spins, as in superfluid helium-3. Neutron-proton pairs could also exist in principle; however, their formation is not strongly favored in the asymmetric nuclear matter of neutron stars.

\subsubsection{Neutron pairing gap in uniform neutron matter at zero temperature}

A central quantity in BCS theory is the gap function, which is related to the binding energy of a pair. Neglecting for the time being nuclear clusters in the inner crust and considering pure neutron matter, the gap equations at a given number density $n$ and at zero temperature read, in the simplest approximation [346],

$$
\Delta(\boldsymbol{k})=-\frac{1}{2} \int \frac{d^{3} \boldsymbol{k}^{\prime}}{(2 \pi)^{3}} \widetilde{V}_{\boldsymbol{k}, \boldsymbol{k}^{\prime}} \frac{\Delta\left(\boldsymbol{k}^{\prime}\right)}{\sqrt{\left(\epsilon\left(\boldsymbol{k}^{\prime}\right)-\mu\right)^{2}+\Delta\left(\boldsymbol{k}^{\prime}\right)^{2}}}
$$

together with

$$
n \equiv \frac{k_{\mathrm{F}}^{3}}{3 \pi^{2}}=\int \frac{d^{3} \boldsymbol{k}}{(2 \pi)^{3}}\left(1-\frac{\epsilon(\boldsymbol{k})-\mu}{\sqrt{(\epsilon(\boldsymbol{k})-\mu)^{2}+\Delta(\boldsymbol{k})^{2}}}\right)
$$

where $\widetilde{V}_{\boldsymbol{k}, \boldsymbol{k}^{\prime}}$ is the matrix element of the pairing interaction between time-reversed plane-wave states with wave vectors $\boldsymbol{k}$ and $\boldsymbol{k}^{\prime}, \mu$ is the chemical potential and $\epsilon(\boldsymbol{k})$ is the single particle energy. Whenever the ratio $\Delta(\boldsymbol{k}) / \mu$ is small, the concept of a Fermi surface remains well defined and the two equations can be decoupled by setting $\mu=\epsilon\left(k_{\mathrm{F}}\right) \equiv \epsilon_{\mathrm{F}}$.

Since the kernel in the gap integral peaks around the chemical potential $\mu \simeq \epsilon_{\mathrm{F}}$, let us suppose that the pairing matrix elements $\widetilde{V}_{\boldsymbol{k}, \boldsymbol{k}^{\prime}} \simeq \widetilde{V}_{k_{\mathrm{F}}, k_{\mathrm{F}}}$ remain constant within $|\epsilon(k)-\mu|<\epsilon_{\mathrm{C}}$ and zero elsewhere; $\epsilon_{\mathrm{C}}$ is a cutoff energy. With this schematic interaction, the gap function becomes independent of $\boldsymbol{k}$. In conventional superconductors, the electron pairing is conveyed by vibrations in the ion lattice. The ion plasma frequency thus provides a natural cutoff $\epsilon_{\mathrm{C}}=\hbar \omega_{\mathrm{pi}}$ (see Section 8.1). In the nuclear case however, there is no a priori choice of $\epsilon_{\mathrm{C}}$. A cutoff can still be introduced in the BCS equations, provided the pairing interaction is suitably renormalized, as shown by Anderson \& Morel [11]. The BCS gap equations (128) can be solved analytically in the weak coupling approximation, assuming that the pairing interaction is small, $g(\mu) \widetilde{V}_{k_{\mathrm{F}}, k_{\mathrm{F}}} \ll 1$, where $g(\epsilon)$ is the

Living Reviews in Relativity

http://www . livingreviews . org//rr-2008-10 
density of single particle states at the energy $\epsilon$. Considering that $g(\epsilon)$ remains constant in the energy range $|\epsilon-\mu|<\epsilon_{\mathrm{C}}$, the gap $\Delta\left(k_{\mathrm{F}}\right) \equiv \Delta_{\mathrm{F}}$ at the Fermi momentum $k_{\mathrm{F}}$ is given by

$$
\Delta_{\mathrm{F}} \simeq 2 \epsilon_{\mathrm{C}} \exp \left(\frac{2}{g(\mu) \widetilde{V}_{k_{\mathrm{F}}, k_{\mathrm{F}}}^{\mathrm{C}}}\right),
$$

where the superscript $\mathrm{C}$ is to remind us that the strength of the pairing interaction depends on the cutoff. This expression is usually not very good for nuclear matter because the pairing interaction is strong. Nevertheless it illustrates the highly nonperturbative nature of the pairing gap. It also shows that the lower the density of states, the lower the gap. Consequently the neutron pairing gap in neutron star crust is expected to be smaller inside the nuclear clusters (discrete energy levels) than outside (continuous energy spectrum) as confirmed by detailed calculations (see Section 8.2.3).

The pairing gap obtained by solving Equations (128) and (129) for neutron matter using a bare nucleon-nucleon potential and assuming a free Fermi gas single particle spectrum

$$
\epsilon(k)=\frac{\hbar^{2} k^{2}}{2 m_{n}},
$$

where $m_{n}$ is the neutron mass, is almost independent of the nucleon-nucleon potential (provided it fits scattering data) and is shown in Figure 45.

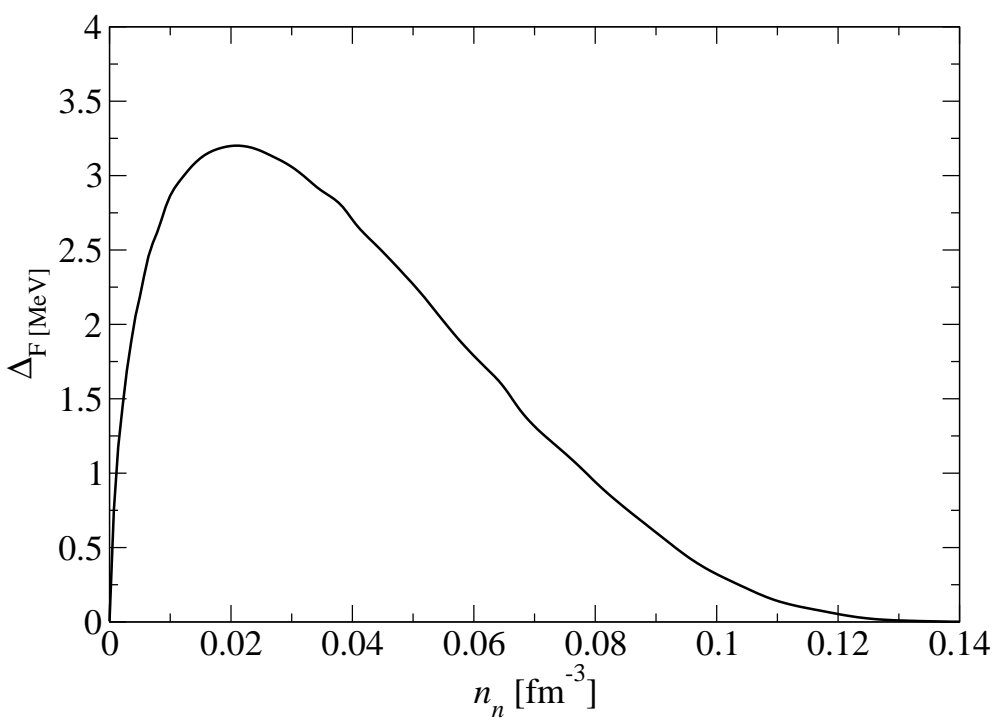

Figure 45: Typical ${ }^{1} S_{0}$ pairing gap in pure neutron matter as a function of the neutron number density, obtained in the BCS approximation with a bare nucleon-nucleon potential and the free energy spectrum (taken from Figure 7 of [271]).

As can be seen, the neutron pairs are most strongly bound at neutron densities around $n_{n} \simeq$ $0.02 \mathrm{fm}^{-3}$. At higher densities, the pairing gap decreases due to the short range repulsive part of the nucleon-nucleon interaction. The pairing gap is almost independent of the nucleon-nucleon potential. The reason is that nucleon-nucleon potentials are constrained to reproduce the experimental phase shifts up to scattering energies of order $E_{\text {lab }} \sim 350 \mathrm{MeV}$, which corresponds to neutron densities of order $n_{n} \simeq 0.3 \mathrm{fm}^{-3}$. In fact, it can be shown that the pairing gap is completely determined by the experimental ${ }^{1} S_{0}$ nucleon-nucleon phase shifts [136]. At small relative 
momenta $k$, the neutron-neutron ${ }^{1} S_{0}$ phase shifts $\delta(k)$ are well approximated by the expansion

$$
k \cot \delta(k)=-\frac{1}{a_{n n}}+\frac{1}{2} r_{n n} k^{2},
$$

where $a_{n n}=-18.5 \pm 0.3 \mathrm{fm}$ and $r_{n n}=2.75 \pm 0.11 \mathrm{fm}$ are the neutron-neutron scattering length and effective range, respectively [381]. Large negative values of the scattering length are associated with attractive interactions, which nearly lead to the existence of a di-neutron. In magnetically-trapped Bose atomic gases, the scattering length can be varied experimentally by tuning the magnetic field [127]. It can be shown that for Fermi wave vectors $k_{\mathrm{F}} \lesssim 0.5 \mathrm{fm}^{-1}\left(n_{\mathrm{b}} \lesssim 4 \times 10^{-3} \mathrm{fm}^{-3}\right)$, the pairing gap $\Delta_{\mathrm{F}}$ is completely determined by these two parameters only [136]. In the zero density limit $k_{\mathrm{F}}\left|a_{n n}\right| \ll 1$ (i.e., $n_{n} \ll 5 \times 10^{-6} \mathrm{fm}^{-3}$ ), the gap equations are exactly solvable and the pairing gap can be expressed analytically by the exact formula [321]

$$
\Delta_{\mathrm{F}}=\frac{8}{\exp (2)} \mu \exp \left(\frac{\pi}{2 k_{\mathrm{F}} a_{n n}}\right)
$$

Let us emphasize that this formula is universal and valid for any fermion system with attractive interactions $\left(a_{n n}<0\right)$. Equation (133) shows that the pairing gap strongly depends on the density and the nucleon-nucleon interaction. It can, thus, be foreseen that modifications of the bare nucleon-nucleon interaction due to medium polarization, which have been neglected, have a dramatic effect. Indeed, it can be rigorously shown [172] that in the low density limit, polarization effects reduce the gap value $(133)$ by a factor of $4^{1 / 3} \exp (1 / 3) \sim 2$, independent of the strength of the interaction!

The gap Equations (128) and (129) solved for the bare interaction with the free single particle energy spectrum, Equation (131), represent the simplest possible approximation to the pairing problem. A more consistent approach from the point of view of the many-body theory, is to calculate the single particle energies in the Hartree-Fock approximation (after regularizing the hard core of the bare nucleon-nucleon interaction). The next step is to "dress" the pairing interaction by medium polarization effects. Calculations have been carried out with phenomenological nucleonnucleon interactions such as the Gogny force [117, 140], that are constructed so as to reproduce some properties of finite nuclei and nuclear matter. Another approach is to derive this effective interaction from a bare nucleon-nucleon potential (two-body and/or three-body forces) using manybody techniques. Still the gap equations of form (128) neglect important many-body aspects.

In many-body theory, the general equations describing a superfluid Fermi system are the Nambu-Gorkov equations [3], in which the gap function $\Delta(\boldsymbol{k}, \omega)$ depends not only on the wave vector $k$ but also on the frequency $\omega$. This frequency dependence arises from dynamic effects. In this framework, it can be shown that BCS theory is a mean field approximation to the many-body pairing problem. The Gorkov equations cannot be solved exactly and some approximations have to be made. Over the past years, this problem has been tackled using different microscopic treatments and approximation schemes. Qualitatively these calculations lead to the conclusion that medium effects reduce the maximum neutron pairing gap compared to the BCS value (note that this includes the possibility that medium effects actually increase the pairing gap for some range of densities). However, these calculations predict very different density dependence of the pairing gap as illustrated in Figure 46.

Before concluding this section, we provide an analytic formula for a few representative neutron pairing gaps, using the following expression proposed by Kaminker et al. [229].

$$
\Delta_{\mathrm{F}}=\theta\left(k_{\max }-k_{\mathrm{F}}\right) \Delta_{0} \frac{k_{\mathrm{F}}^{2}}{k_{\mathrm{F}}^{2}+k_{1}^{2}} \frac{\left(k_{\mathrm{F}}-k_{2}\right)^{2}}{\left(k_{\mathrm{F}}-k_{2}\right)^{2}+k_{3}^{2}},
$$

where $k_{\mathrm{F}}=\left(3 \pi^{3} n_{n}\right)^{1 / 3}$ and $\theta$ is the Heaviside step function $\theta(x)=1$ if $x>0$ and $\theta(x)=0$ otherwise. The parameters $\Delta_{0}, k_{1}, k_{2}, k_{3}$ and $k_{\max }$ are given in Table 5. At low density, the pairing gap $\Delta_{\mathrm{F}} \propto k_{\mathrm{F}}^{2}$ varies roughly as expected, Equation (133), remembering that $\mu \sim \epsilon_{\mathrm{F}} \propto k_{\mathrm{F}}^{2}$.

Living Reviews in Relativity

http://www. livingreviews.org/Irr-2008-10 


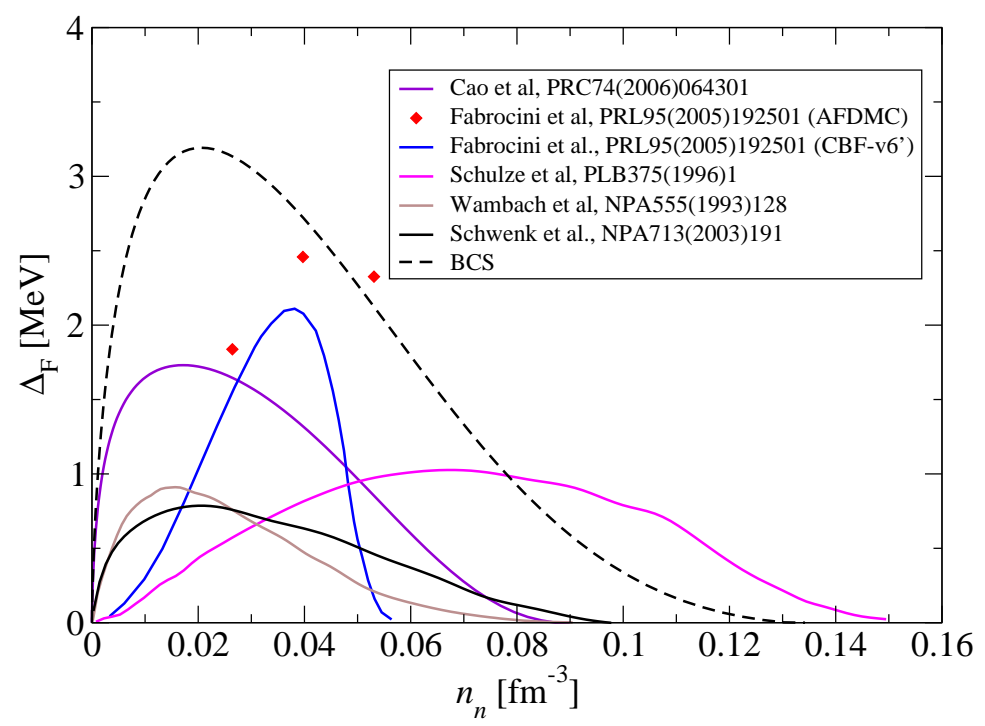

Figure 46: ${ }^{1} S_{0}$ pairing gap in pure neutron matter as a function of the neutron number density obtained from microscopic calculations with different approximations to account for medium effects.

Table 5: Parameters for the analytic formula Equation (134) of a few representative ${ }^{1} S_{0}$ pairing gaps in pure neutron matter: BCS-BCS pairing gap shown in Figure 45, Brueckner - pairing gap of Cao et al. [69] based on diagrammatic calculations (shown in Figure 46) and RG - pairing gap of Schwenk et al. [366] based on the Renormalization Group approach (shown in Figure 46). $\Delta_{0}$ is given in MeV. $k_{1}, k_{2}, k_{3}$ and $k_{\max }$ are given in $\mathrm{fm}^{-1}$.

\begin{tabular}{cccccc}
\hline model & $\Delta_{0}$ & $k_{1}$ & $k_{2}$ & $k_{3}$ & $k_{\max }$ \\
\hline BCS & 910.603 & 1.38297 & 1.57068 & 0.905237 & 1.57 \\
Brueckner & 11.4222 & 0.556092 & 1.38236 & 0.327517 & 1.37 \\
RG & 16.5709 & 1.13084 & 1.47001 & 0.582515 & 1.5 \\
\hline
\end{tabular}




\subsubsection{Critical temperature for neutron superfluidity}

The BCS gap Equations (128) at zero temperature can be generalized to finite temperature $T$ (adopting the standard notation $\beta \equiv 1 / k_{\mathrm{B}} T$ where $k_{\mathrm{B}}$ is the Boltzmann constant)

$$
\Delta(\boldsymbol{k})=-\frac{1}{2} \int \frac{d^{3} \boldsymbol{k}^{\prime}}{(2 \pi)^{3}} \widetilde{V}_{\boldsymbol{k}, \boldsymbol{k}^{\prime}} \frac{\Delta\left(\boldsymbol{k}^{\prime}\right)}{\sqrt{\left(\epsilon\left(\boldsymbol{k}^{\prime}\right)-\mu\right)^{2}+\Delta\left(\boldsymbol{k}^{\prime}\right)^{2}}} \tanh \frac{\beta}{2} \sqrt{\left(\epsilon\left(\boldsymbol{k}^{\prime}\right)-\mu\right)^{2}+\Delta\left(\boldsymbol{k}^{\prime}\right)^{2}} .
$$

Superfluidity disappears whenever the temperature exceeds some critical threshold. Let us remark that isotropic neutron superfluidity can also be destroyed by a sufficiently strong magnetic field, since it would force each spin of a neutron pair to be aligned (as pointed out by Kirszshnits [239]). It can be shown on general grounds that the isotropic pairing gap $\Delta_{\mathrm{F}}(T=0)$ at zero temperature (at Fermi momentum $k_{\mathrm{F}}$ ) and the critical temperature $T_{\mathrm{c}}$ are approximately related by $[36]$

$$
\Delta_{\mathrm{F}}(T=0)=\pi \exp (-\gamma) k_{\mathrm{B}} T_{\mathrm{c}} \simeq 1.76 k_{\mathrm{B}} T_{\mathrm{c}},
$$

where $\gamma$ is the Euler constant. This well-known result of conventional electron superconductivity applies rather well to nucleon superfluidity, especially for densities at which $\Delta_{\mathrm{F}}(0)$ takes its maximum value [271].

The temperature dependence of the pairing gap, for $T \leq T_{\mathrm{c}}$, can be approximately written as $[428]$

$$
\Delta_{\mathrm{F}}(T) \simeq k_{\mathrm{B}} T \sqrt{1-\frac{T}{T_{\mathrm{c}}}}\left(1.456-0.157\left(\frac{T}{T_{\mathrm{c}}}\right)^{-1 / 2}+1.764\left(\frac{T}{T_{\mathrm{c}}}\right)^{-1}\right) .
$$

Zero temperature pairing gaps on the order of $1 \mathrm{MeV}$ are therefore associated with critical temperatures of the order $10^{10} \mathrm{~K}$, considerably larger than typical temperatures inside neutron stars except for the very early stage of their formation. The existence of a neutron superfluid in the inner crust of a neutron star is therefore well established theoretically. Nevertheless the density dependence of the critical temperature predicted by different microscopic calculations differ considerably due to different approximations of the many-body problem. An interesting issue concerns the cooling of neutron stars and the crystallization of the crust: do the neutrons condense into a superfluid phase before the formation of the crust or after?

Figure 47 shows the melting temperature $T_{\mathrm{m}}$ of the inner crust of neutron stars compared to the critical temperature $T_{\mathrm{c}}$ for the onset of neutron superfluidity. The structure of the crust is that calculated by Negele \& Vautherin [303]. The melting temperature has been calculated from Equation (15) with $\Gamma_{\mathrm{m}}=175$. The temperature $T_{\mathrm{c}}$ has been obtained from Equation (136), considering a uniform neutron superfluid, with the density $\tilde{\rho}_{\mathrm{G}}$ of unbound neutrons given by Negele \& Vautherin [303]. Several critical temperatures are shown for comparison. As discussed in Section 8.2.1, the BCS value represents the simplest approximation to the true critical temperature. The other two critical temperatures have been obtained from more realistic pairing-gap calculations, which include medium effects using different many-body approximations. The calculation of Cao et al. [69] is based on diagrammatic calculations, while that of Schwenk et al. [366] relies on the renormalization group.

For the BCS and Brueckner calculations of the pairing gap, in the density range of $\sim 10^{12}-10^{14} \mathrm{~g} \mathrm{~cm}^{-3}$, the neutrons may become superfluid before the matter crystallizes into a solid crust. As discussed in Section 8.3.2, as a result of the rotation of the star, the neutron superfluid would be threaded by an array of quantized vortices. These vortices might affect the crystallization of the crust by favoring nuclear clusters along the vortex lines, as suggested by Mochizuki et al. [293]. On the contrary, the calculations of Schwenk et al. [366] indicate that, at any density, the solid crust would form before the neutrons become superfluid. Recently, it has also been shown, by taking into account the effects of the inhomogeneities on the neutron superfluid, that in the shallow layers

Living Reviews in Relativity

http://www. livingreviews.org/Irr-2008-10 
of the inner crust, the neutrons might remain in the normal phase even long after the formation of the crust, when the temperature has dropped below $10^{9} \mathrm{~K}[294]$.

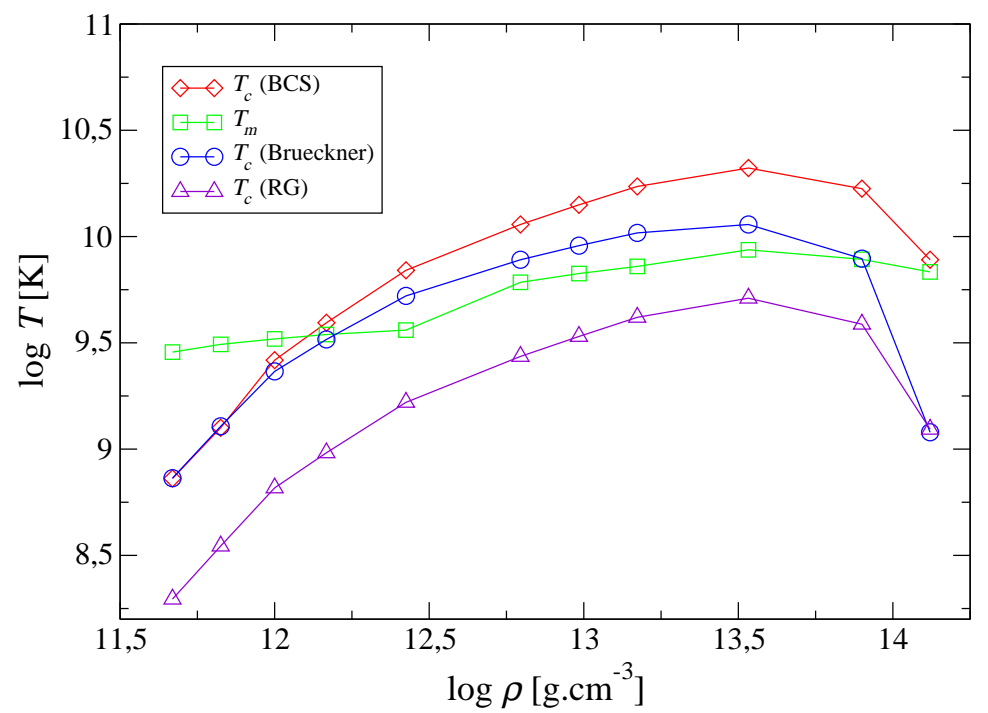

Figure 47: Melting temperature $T_{\mathrm{m}}$ of the crust and critical temperature $T_{\mathrm{c}}$ for the onset of neutron superfluidity as a function of the density $\rho$. The model of the inner crust is based on Negele \& Vautherin[303]. Three representative cases (as shown in Figure 46) are: the BCS pairing gap $\Delta_{\mathrm{F}}$ and the more realistic pairing gaps of Cao et al. [69] and Schwenk et al. [366].

\subsubsection{Pairing gap in neutron star crusts}

In this section, we will discuss the effects of the nuclear clusters on the pairing properties of the neutron superfluid in neutron star crusts. The relative importance of these effects is determined by the coherence length, defined as the root mean square radius of the pair wave function. Broadly speaking, the coherence length represents the size of a neutron pair. This is an important length scale, which determines many properties of the superfluid. For instance, the coherence length is of the order of the size of the superfluid vortex cores. According to Anderson's theorem [114], the effects of the inhomogeneities (here - nuclear clusters) on the neutron superfluid are negligible whenever the coherence length is much larger than the characteristic size of the inhomogeneities. Assuming weak coupling, the coherence length can be roughly estimated from Pippard's expression

$$
\xi=\frac{\hbar^{2} k_{\mathrm{F}}}{\pi m_{n} \Delta_{\mathrm{F}}},
$$

where $\Delta_{\mathrm{F}}$ is the neutron pairing gap at the Fermi momentum $k_{\mathrm{F}}$ (see Section 8.2.1). This expression gives only a lower bound for the coherence length, since medium effects tend to reduce the pairing correlations as discussed in Section 8.2.1. Nevertheless, this estimate is rather close to the value obtained in more detailed calculations [111, 283]. As can be seen in Figure 48, the coherence length is smaller than the lattice spacing ${ }^{6}$ except for the densest layers of the crust. Consequently the effects of the solid crust on neutron superfluidity cannot be neglected. This situation is in sharp contrast to that encountered in ordinary type I superconductors, where the electron Cooper pairs

\footnotetext{
${ }^{6}$ For a body-centered-cubic lattice, the lattice spacing $a$ is related to the Wigner-Seitz radius $R_{\text {cell }}$ by $a=$ $2(\pi / 3)^{1 / 3} R_{\text {cell }}$.
} 
are spatially extended over mesoscopic distances of $\sim 10^{3}-10^{4} \AA$ and as a result the pairing gap is nearly insensitive to the details of the atomic crystal structure, since the typical lattice spacing is of order a few $\AA$. In Figure 48 we also displayed the mean inter-neutron spacing defined by

$$
d_{n}=2\left(\frac{3}{4 \pi n_{n}}\right)^{1 / 3}
$$

In the denser layers of the crust, the coherence length is smaller than the mean inter-neutron spacing, suggesting that the neutron superfluid is a Bose-Einstein condensate of strongly-bound neutron pairs, while in the shallower layers of the inner crust the neutron superfluid is in a BCS regime of overlapping loosely-bound pairs. Quite remarkably, for screened pairing gaps like those of Schwenk et al.[366], the coherence length is larger than the mean inter-neutron spacing in the entire inner crust, so that in this case, at any depth, neutron superfluid is in the BCS regime.

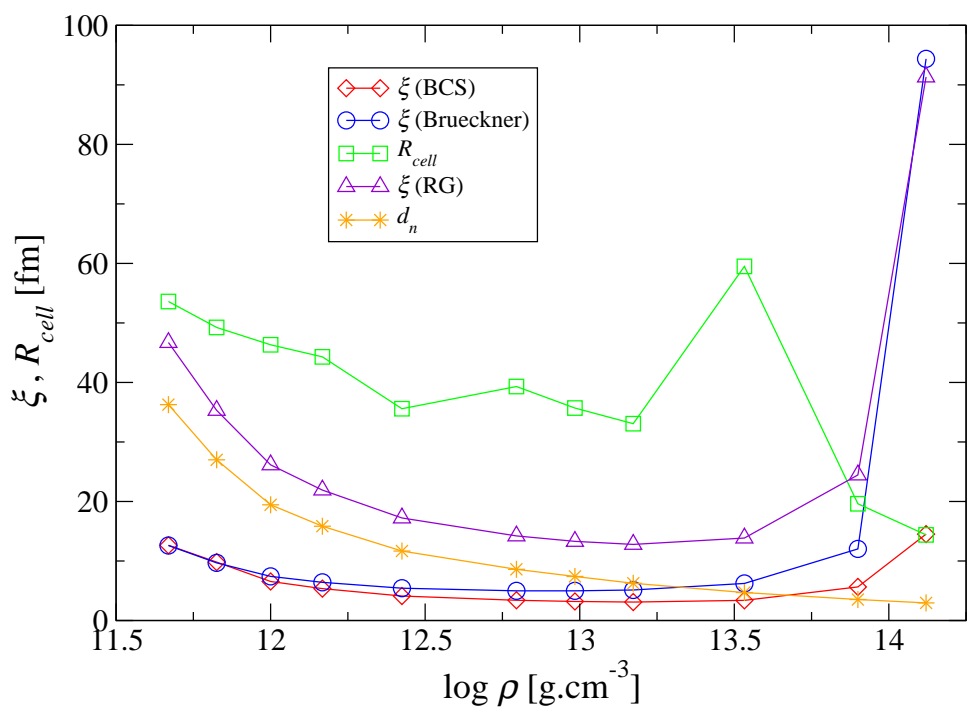

Figure 48: Pippard's coherence length for the neutron star crust model of Negele \& Vautherin[303]. The coherence length has been calculated from Equation (138), assuming that the neutron superfluid is uniform with the density of unbound neutrons denoted by $\tilde{\rho}_{\mathrm{G}}$ in [303]. Three representative cases have been considered: the BCS pairing gap $\Delta_{\mathrm{F}}$, and the more realistic pairing gaps of Cao et al. [69] and Schwenk et al. [366]. The gaps are shown in Figure 46. For comparison, we also show the radius $R_{\text {cell }}$ of the Wigner-Seitz sphere and the mean inter-neutron spacing $d_{n}$.

Since the formulation of the BCS theory, considerable theoretical efforts have been devoted to the microscopic calculation of pairing gaps in uniform nuclear matter using the many body theory. On the other hand, until recently the superfluidity in neutron star crusts has not attracted much attention despite its importance in many observational phenomena like pulsar glitches (see Section 12). The pairing correlations in an inhomogeneous superfluid system can be described in terms of a pairing field $\Delta(r)$. In early studies $[59,107,135]$ the pairing field has been calculated assuming that the matter is locally homogeneous (local density approximation). Such calculations predict, in particular, that the value of the pairing field inside the nuclear clusters is almost the same for different layers of the crust. The reason lies in the nuclear saturation: the density inside heavy nuclei is essentially constant, independent of the number of bound nucleons. In some cases, the pairing field was found to vanish inside the clusters [135]. The local density approximation is valid if the coherence length is smaller than the characteristic scale of density variations. However,

Living Reviews in Relativity

http://www. livingreviews.org/Irr-2008-10 
this condition is never satisfied in the crust. As a result, the local density approximation overestimates the spatial variation of the pairing field. Due to "proximity effects", the free superfluid neutrons induce pairing correlations of the bound neutrons inside clusters and vice versa leading to a smooth spatial variation of the neutron pairing field [38]. As a remarkable consequence, the value of the neutron pairing field outside (resp. inside) the nuclear clusters is generally smaller (resp. larger) than that obtained in uniform neutron matter for the same density [31]. In particular, the neutrons inside the clusters are also superfluid. The neutron superfluid in the crust should, therefore, be thought of as an inhomogeneous superfluid rather than a superfluid flowing past clusters like obstacles. The effects of nuclear clusters on neutron superfluidity have been investigated in the Wigner-Seitz approximation by several groups. These calculations have been carried out at the mean field level with realistic nucleon-nucleon potentials [37], effective nucleon-nucleon interactions [38, 295, 361, 360, 237] and with semi-microscopic energy functionals [29, 30, 31]. Examples are shown in Figure 49. The effects of medium polarization have been considered by the Milano group [169, 413], who found that these effects lead to a reduction of the pairing gap, as in uniform neutron matter. However, this quenching is less pronounced than in uniform matter due to the presence of nuclear clusters. Apart from uncertainties in the pairing interaction, it has recently been shown $[30,31]$ that the pairing field is very sensitive to the choice of boundary conditions, especially in the bottom layers of the crust (as also found by the other groups). Consequently, the results obtained in the Wigner-Seitz approximation should be interpreted with caution, especially when calculating thermodynamic quantities like the neutron specific heat, which depends exponentially on the gap.

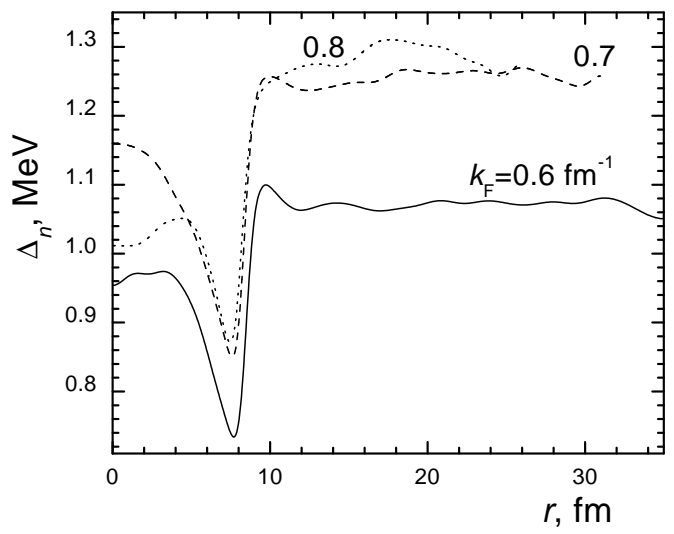

Figure 49: Neutron pairing fields in the inner crust, calculated by Baldo et al. [32]. Results are shown inside the Wigner-Seitz sphere. $k_{\mathrm{F}}$ is the average Fermi momentum defined by $k_{\mathrm{F}}=\left(3 \pi^{2} n_{\mathrm{b}}\right)^{1 / 3}$, where $n_{\mathrm{b}}$ is the baryon density.

\subsection{Superfluid hydrodynamics}

\subsubsection{Superflow and critical velocity}

The basic property of superfluid is that it can flow without dissipation. In a normal fluid, friction and viscosity arise because particles are randomly scattered. Such scattering events are forbidden in superfluid because energy and momentum cannot be simultaneously conserved. The key argument of Landau is that in a superfluid like helium-4, the particles are strongly correlated so that the concept of single particles becomes meaningless. However, at low enough temperatures, the system is still assumed to be described in terms of noninteracting "quasiparticles", which do not correspond to material particles but to many-body motions (excitations). The energy spectrum of these 
quasiparticles can be very different from that of single particles. Using this idea, Landau [247] was able to explain the origin of nondissipative superflow and the existence of a critical velocity beyond which superfluidity disappears. The argument is the following. Let us consider a macroscopic body of mass $M$ flowing through the superfluid. At low temperatures, its velocity $\boldsymbol{V}$ can only be changed in scattering processes where one or more quasiparticles are created, assuming that the flow is not turbulent. For a quasiparticle of energy $E(p)$ and momentum $p$ to be created, energy conservation implies that

$$
\frac{1}{2} M V^{2}>\frac{1}{2} M V^{\prime 2}+E(p),
$$

where $\boldsymbol{V}^{\prime}$ is the body's velocity after the event. However, momentum must also be conserved,

$$
M V=M V^{\prime}+p .
$$

These two conditions can only be satisfied if

$$
E(p)<\boldsymbol{V} \cdot \boldsymbol{p}-\frac{p^{2}}{2 M} .
$$

Since the perturbing body contains a macroscopic collection of particles, the mass $M$ is very large so that the second term can be neglected. The resulting inequality cannot be satisfied unless the velocity exceeds some critical value

$$
V_{c}=\min \left\{\frac{E(p)}{p}\right\},
$$

where $\min \{x\}$ is the smallest value of $x$ in a set $\{x\}$. This means that for velocities smaller than $V_{c}$, the creation of quasiparticles is forbidden and therefore the fluid flows without dissipation. In a normal liquid, the single particle energy is given by an expression of the form

$$
E(p)=\frac{p^{2}}{2 m},
$$

where $m$ is an effective mass suitably renormalized to include many-body effects. Consequently, the critical velocity, according to Landau's criterion (143), is zero, $V_{c}=0$. Since liquid helium-4 is superfluid, Landau [247, 246] postulated a different energy spectrum. At low momenta, the quasiparticle excitations are sound waves (phonons) as illustrated in Figure 50. The dispersion relation is thus given by

$$
E(p)=c_{\mathrm{s}} p,
$$

where $c_{\mathrm{s}}$ is a sound speed.

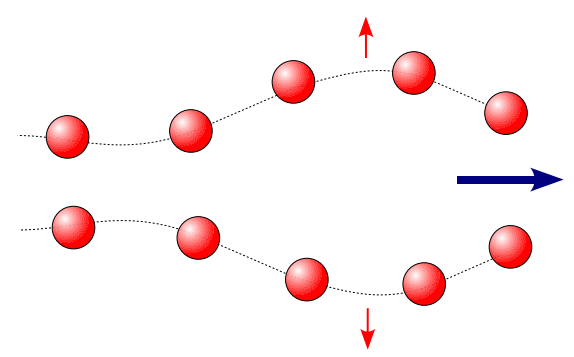

Figure 50: Schematic picture illustrating collective motions of particles associated with a low momentum quasiparticle (phonon). 
At very high momenta, the dispersion relation coincides with that of a normal liquid, Equation (144). In between, the dispersion relation exhibits a local minimum and is approximately given by

$$
E(p)=\Delta_{\mathrm{r}}+\frac{\left(p-p_{0}\right)^{2}}{2 m_{\mathrm{r}}}
$$

The quasiparticles associated with this minimum were dubbed "rotons" by I.E. Tamm as reported by Landau [247]. Landau postulated that these rotons are connected with a rotational velocity flow, hence the name. These rotons arise due to the interactions between the particles. Feynman [145] argued that a roton can be associated with the motion of a single atom. As the atom moves through the fluid, it pushes neighboring atoms out of its way forming a ring of particles rotating backwards as illustrated in Figure 51. The net result is a vortex ring of an atomic size.

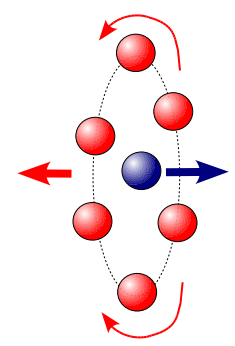

Figure 51: Schematic picture illustrating collective motions of particles associated with a roton quasiparticle according to Feynman's interpretation.

The roton local minimum has also been interpreted as a characteristic feature of density fluctuations marking the onset of crystallization [200, 334, 307]. According to Nozières [307], rotons are "ghosts of Bragg spots". Landau's theory has been very successful in explaining the observed properties of superfluid helium-4 from the postulated energy spectrum of quasiparticles.

In weakly interacting dilute Bose gases, as in ultra-cold Bose atomic gases, the energy of the quasiparticles are given by (see for instance Section 21 of Fetter \& Walecka [142])

$$
E(p)=\sqrt{\left(\frac{p^{2}}{2 m}\right)^{2}+p^{2} c_{\mathrm{s}}^{2}},
$$

where $c_{\mathrm{s}}$ is the speed of sound. At low momentum $p$, it reduces to Equation (145), while at high momentum it tends to Equation (144). Dilute Bose gases, thus, have only phonon excitations. Quite remarkably, the ideal Bose gas (which is characterized by the dispersion relation (147) with $\left.c_{\mathrm{s}}=0\right)$ exhibits a Bose-Einstein condensation at low enough temperatures, but is not superfluid since its critical velocity is equal to zero.

Owing to the specific energy spectrum of quasiparticles in both atomic Bose gases and helium-4, the critical velocity does not vanish, thus explaining their superfluid properties. Landau's critical velocity, Equation (143), of superfluid helium-4 due to the emission of rotons, is given by

$$
V_{c}=\frac{\Delta_{\mathrm{r}}}{p_{0}} \simeq 60 \mathrm{~m} \mathrm{~s}^{-1}
$$

This value has been confirmed by ion propagation experiments [137]. However, in most experiments, much smaller critical velocities are measured due to the existence of other kinds of excitations. The critical velocity of atomic Bose gases has also been measured, using laser beams instead of a macroscopic object [235]. Again, velocities smaller than Landau's velocity (which is equal to the velocity of sound in this case) have been found. 
The previous discussion of the critical velocity of Bose liquids can be easily extended to fermionic superfluids. In the BCS theory, fermions form bound pairs, which undergo Bose condensation when the temperature falls below a critical temperature (Section 8.2). The quasiparticle energies for a uniform Fermi system are given by

$$
E(p)=\sqrt{(\epsilon(p)-\mu)^{2}+\Delta(p)^{2}},
$$

where $p=\hbar k, \epsilon(p)$ is the single particle energy, $\mu$ the chemical potential and $\Delta(p)$ the pairing gap at momentum $p$. According to Landau's argument, the critical velocity (143) is equal to

$$
V_{c}=\frac{\Delta_{\mathrm{F}}}{\hbar k_{\mathrm{F}}}
$$

This expression can be derived more rigorously from the microscopic BCS theory [35]. It shows that a system of fermions is superfluid (i.e. the critical velocity is not zero) whenever the interactions are attractive, so that the formation of pairs becomes possible. It is also interesting to note that the BCS spectrum can be interpreted in terms of rotons. Indeed, expanding Equation (149) around the minimum leads, to lowest order, to an expression similar to Equation (146). In this case, $p_{0}$ is obtained by solving $\epsilon(p)=\mu$. The other parameters are given by $\Delta_{\mathrm{r}}=\Delta\left(p_{0}\right)$ and $m_{\mathrm{r}}=\Delta\left(p_{0}\right) / v_{0}^{2}$ where $v_{0}=d \epsilon / d p$ is the group velocity evaluated at $p_{0}$.

The presence of an "external" potential affects superfluidity. This issue has recently attracted a lot of theoretical, as well as experimental, interest in the field of optically-trapped ultra-cold atomic Bose gases [297]. It is also relevant in the context of neutron stars, where the solid crust is immersed in a neutron superfluid (and possibly a proton superconductor in the liquid crystal mantle, where nuclear "pastas" could be present; see Section 3.3). In the BCS approximation (128), considering a periodic potential (induced by the solid crust in neutron stars), the quasiparticle energies still take a form similar to Equation (149). However, the dependence on the momentum is no more isotropic. As shown by Carter, Chamel \& Haensel [77], Equation (150) for the critical velocity should then be replaced by

$$
V_{c}=\min _{\mathrm{FS}}\left\{\frac{\Delta(\boldsymbol{k})}{m_{\star} v(\boldsymbol{k})}\right\}
$$

where $\boldsymbol{k}$ is the Bloch wave vector (Section 3.2.4), $\boldsymbol{v}(\boldsymbol{k})=\hbar^{-1} \boldsymbol{\nabla}_{\boldsymbol{k}} \epsilon(\boldsymbol{k})$ is the group velocity of the fermions and $m_{\star}$ is an effective mass, which arises from the interactions between the particles and the lattice as discussed in Section 8.3.6. The subscript FS means that the minimum is to be searched on the Fermi surface ${ }^{7}$. In the neutron star crust, the effective mass of the unbound neutrons could be very large $m_{\star} \gg m_{n}$, as shown by Chamel [90, 91]. In the limit of homogeneous matter, the single particle energies are given by an expression of the form (144) with $m=m_{n}$ and $\boldsymbol{p}=\hbar \boldsymbol{k}$. Consequently, $m_{\star}=m_{n}$ and $\boldsymbol{v}=\hbar \boldsymbol{k} / m_{n}$, so that Equation (151) reduces to Equation (150) using the extended Brillouin zone scheme. Equation (151) shows that superfluidity disappears whenever the pairing gap vanishes at some point on the Fermi surface.

The real critical velocity is expected to be smaller than that given by Equation (151) due to finite temperature and many-body effects beyond the mean field. Likewise, the critical velocity of superfluid helium-4 obtained in Landau's quasiparticle model is only an upper bound because in this model, the quasiparticles are assumed to be noninteracting. The experimentally-measured critical velocities are usually much smaller, in particular, due to the nucleation of vortices. Indeed, relative motions between superfluid and the vortices lead to mutual friction forces and, hence, to dissipative effects. In general, any curved vortex line does not remain at rest in the superfluid reference frame and, therefore, induces dissipation. Feynman [143] derived the critical velocity

\footnotetext{
${ }^{7}$ The Fermi surface is the surface in $k$-space defined by $\epsilon(\boldsymbol{k})=\mu$. Note that, in general, it is not spherical.
} 
associated with the formation of a vortex ring in a channel of radius $R$,

$$
V_{c} \simeq \frac{\hbar}{m R} \log \left(\frac{R}{r_{v}}\right),
$$

where $r_{v}$ is the radius of the vortex core. This result shows that the critical velocity depends, in general, on experimental set-up. More generally, the critical velocity scales like $V_{c} \propto \hbar / m L$, where $L$ is a characteristic length scale in the experiment. The theoretical determination of the breakdown of superfluidity is still an open issue, which requires a detailed understanding of superfluid dynamics and, in particular, the dynamics of vortices.

\subsubsection{Rotating superfluid and vortices}

Superfluidity is closely related to the phenomenon of Bose-Einstein condensation as first envisioned by Fritz London [272]. In the superfluid phase, a macroscopic collection of particles condense into the lowest quantum single particle state, which (for a uniform system) is a plane wave state with zero momentum (therefore a constant). Soon after the discovery of the superfluidity of liquid helium, Fritz London introduced the idea of a macroscopic wave function $\Psi(\boldsymbol{r})$, whose squared modulus is proportional to the density $n_{\Psi}$ of particles in the condensate. This density $n_{\Psi}$, which should not be confused with the superfluid density $n_{\mathcal{S}}$ introduced in the two-fluid model of superfluids (Section 8.3.6), can be rigorously defined from the one-particle density matrix [322]. The wave function is defined up to a global phase factor. The key distinguishing feature of a superfluid is the symmetry breaking of this gauge invariance by imposing that the phase $\phi(\boldsymbol{r})$ be local. The macroscopic wave function thus takes the form

$$
\Psi(\boldsymbol{r})=\sqrt{n_{\Psi}} e^{\mathrm{i} \phi(\boldsymbol{r})} .
$$

Applying the momentum operator $-\mathrm{i} \hbar \nabla$ to this wave function shows that superfluid carries a net momentum (per superfluid particle)

$$
\boldsymbol{p}=\hbar \nabla \phi
$$

This implies that the superflow is characterized by the condition

$$
\nabla \times p=0
$$

In the absence of any entrainment effects (as discussed in Section 8.3.6), the momentum is given by $\boldsymbol{p}=m \boldsymbol{v}$, where $m$ is the mass of the superfluid "particles" 8 and $\boldsymbol{v}$ is the velocity of superfluid. Equation (155) thus implies that the flow is irrotational. This means, in particular, that a superfluid in a rotating bucket remains at rest with respect to the laboratory reference frame. However, this Landau state is destroyed whenever the rotation rate exceeds the critical threshold for the formation of vortices given approximately by Equation (152). Experiments show that the whole superfluid then rotates like an ordinary fluid. The condition (155) can therefore be locally violated as first suggested by Onsager [310] and discussed by Feynman [143]. Indeed, since the phase of the macroscopic wave function is defined modulo $2 \pi$, the momentum circulation over any closed path is quantized

$$
\oint \boldsymbol{p} \cdot \boldsymbol{d} \boldsymbol{\ell}=N h
$$

where $N$ is any integer and $h$ is Planck's constant. Equation (156) is simply the Bohr-Sommerfeld quantization rule. This rule can be equivalently deduced from the following argument. Considering

\footnotetext{
${ }^{8}$ In the case of fermionic superfluids, the superfluid particles are fermion pairs.
} 
superfluid as a macroscopic quantum state, its momentum $p$ is given by $p=h / \lambda$, where $\lambda$ is the de Broglie wavelength. The quantization rule (156) thus follows from the requirement that the length of any closed path must be an integral multiple of the de Broglie wavelength.

In a rotating superfluid the flow quantization implies the formation of vortex lines, each carrying a quantum $\hbar$ of angular momentum, the quantum number $N$ being the number of vortices (the formation of a single vortex carrying all the angular momentum is not energetically favored). The size of the core of a vortex line is roughly on the order of the superfluid coherence length (see Section 8.2.3 for estimates of the coherence length). In some cases, however, it may be much smaller [110, 134], so that the coherence length is only an upper bound on the vortex core size. In the presence of vortices, Equation (155) must therefore be replaced by

$$
\nabla \times \boldsymbol{p}=m \boldsymbol{\kappa},
$$

$\boldsymbol{\kappa}$ being the circulation of the $N$ vortex lines. The existence of quantized vortices was demonstrated by Vinen [415] in 1961, however, they were not observed until much later, in 1974 at Berkeley [423, 432]. At length scales much larger than the superfluid coherence length, the finite size of the vortex core can be neglected and the circulation is, thus, given by

$$
\boldsymbol{\kappa}=\sum_{\alpha=1}^{N} \delta^{(2)}\left(\boldsymbol{r}-\boldsymbol{r}_{\boldsymbol{\alpha}}\right) \boldsymbol{\kappa}_{\boldsymbol{\alpha}},
$$

where $\delta^{(2)}$ is the two-dimensional Dirac distribution, and $\boldsymbol{\kappa}_{\boldsymbol{\alpha}}$ is the circulation of a given vortex $\alpha$

$$
\boldsymbol{\kappa}_{\boldsymbol{\alpha}}=\hat{\boldsymbol{\kappa}}_{\boldsymbol{\alpha}} \frac{1}{m} \oint \boldsymbol{p} \cdot \boldsymbol{d} \boldsymbol{\ell}=\frac{h}{m} \hat{\boldsymbol{\kappa}}_{\boldsymbol{\alpha}}
$$

$\hat{\boldsymbol{\kappa}}_{\boldsymbol{\alpha}}$ being a unit vector directed along the vortex line. Equation (157) is formally similar to Ampere's law in magnetostatics. The momentum $\boldsymbol{p}$ induced by the vortex lines is, thus, given by the BiotSavart equation, substituting $1 / c$ with the mass $m$ divided by $4 \pi$ in Gaussian cgs units (or replacing the magnetic permeability $\mu_{0}$ by the mass $m$ in SI units) and the electric current $I$ by $\kappa$,

$$
\boldsymbol{p}(\boldsymbol{r})=\frac{m}{4 \pi} \int \mathrm{d} r^{\prime} \boldsymbol{\kappa}\left(\boldsymbol{r}^{\prime}\right) \times \frac{\boldsymbol{r}-\boldsymbol{r}^{\prime}}{\left|r-r^{\prime}\right|^{3}},
$$

where the integral is taken along the vortex lines as shown in Figure 52. The analogy between hydrodynamics and magnetostatics shows, in particular, that a vortex ring should move along its symmetry axis with a velocity inversely proportional to its radius.

As shown by Tkachenko [405, 406], quantized vortices tend to arrange themselves on a regular triangular array. Such patterns of vortices have been observed in superfluid helium and more recently in atomic Bose-Einstein condensates. The intervortex spacing $d_{v}$ is given by

$$
d_{v}=\sqrt{\frac{h}{\sqrt{3} m \Omega}},
$$

where $\Omega$ is the angular frequency. At length scales much larger than the intervortex spacing $d_{v}$, as a result of the superposition of the flow pattern of all the vortex lines, superfluid flow mimics rigid body rotation. Since at this scale a fluid element is threaded by many vortex lines, it is relevant to smoothly average the hydrodynamic equations governing the flow of the superfluid. In particular, Equation (157) now reads

$$
\nabla \times \boldsymbol{p}=m n_{v} \boldsymbol{\kappa},
$$

where $n_{v}$ is the surface density of vortices given (in the absence of entrainment effects) by

$$
n_{v}=\frac{m \Omega}{\pi \hbar}
$$

Living Reviews in Relativity

http://www. livingreviews.org/lrr-2008-10 


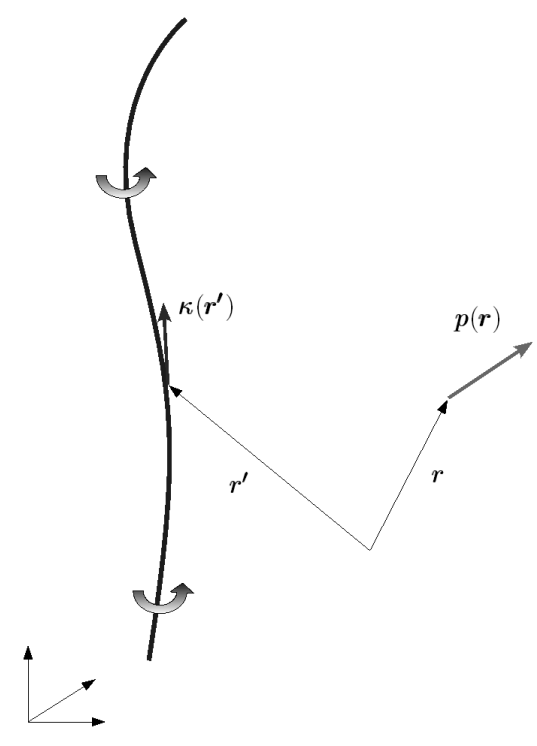

Figure 52: Momentum $\boldsymbol{p}(\boldsymbol{r})$ induced at a position $\boldsymbol{r}$ by the vortex line with circulation $\boldsymbol{\kappa}$.

and the vector $\boldsymbol{\kappa}$, whose norm is equal to $h / m$, is aligned with the average angular velocity (generalization of Equation (163) to account for entrainment effects is discussed in Section 10.4).

\subsubsection{Type II superconductors and magnetic flux tubes}

Let us remark that the condition (155) for superfluids also applies to superconductors, like the proton superconductor in the liquid core and possibly in the "pasta" mantle of neutron stars (Section 3.3). The momentum of a superconductor is given by $\boldsymbol{p} \equiv m \boldsymbol{v}+q \boldsymbol{A}$ (in this section, we use SI units), where $m, q$, and $v$ are the mass, electric charge and velocity of "superconducting" particles respectively ${ }^{9}$, and $\boldsymbol{A}$ the electromagnetic potential vector. Introducing the density $n$ of superconducting particles and their electric current density (referred to simply as "supercurrent") $\mathcal{J}=n q \boldsymbol{v}$, Equation (155) leads to the London equation

$$
\nabla \times \mathcal{J}=-\frac{n q^{2}}{m} \boldsymbol{B}
$$

where $\boldsymbol{B}=\boldsymbol{\nabla} \times \boldsymbol{A}$ is the magnetic field induction. According to the Bohr-Sommerfeld quantization rule (156), the London Equation (164) corresponds to $N=0$. Situations with $N>0$ are encountered in type II superconductors. Considering a closed contour outside a sample of such a superconductor for which $\mathcal{J}=0$ and integrating the momentum $\boldsymbol{p}$ along this contour, leads to the quantization of the total magnetic flux into $N$ flux tubes

$$
\oint \boldsymbol{A} \cdot \boldsymbol{d} \boldsymbol{\ell}=\frac{h N}{q} .
$$

\footnotetext{
${ }^{9}$ For a proton superconductor, $m=2 m_{p}$ and $q=2 e$, where $m_{p}$ and $e$ are the proton mass and proton electric charge, respectively.
} 
These flux tubes tend to arrange themselves into a triangular lattice, the Abrikosov lattice, with a spacing given by

$$
d_{v}=\sqrt{\frac{2 h}{\sqrt{3} q B}} .
$$

Averaging at length scales much larger than $d_{v}$, the surface density of flux tubes is given by

$$
n_{v}=\frac{q B}{h}
$$

\subsubsection{Superfluid vortices and magnetic flux tubes in neutron stars}

For neutron superfluid in neutron stars, superfluid particles are neutron pairs, so that $m=2 m_{n}$. As early as in 1964, Ginzburg \& Kirzhnits [161, 162] suggested the existence of quantized vortex lines inside neutron stars. The critical velocity for the nucleation of vortices can be roughly estimated from $V_{c} \sim \hbar / m_{n} R$ where $R$ is the radius of a neutron star. For $R=10 \mathrm{~km}, V_{c} \sim 10^{3} \mathrm{fm} / \mathrm{s}$, which is, by several orders of magnitude, smaller than characteristic velocities of matter flows within the star. The interior of neutron stars is thus threaded by a huge number of vortices. The intervortex spacing is

$$
d_{v} \simeq 3.4 \times 10^{-3} \sqrt{\frac{10^{2} \mathrm{~s}^{-1}}{\Omega}} \mathrm{cm},
$$

which is much larger than the coherence length, so that the assumption of infinitely-thin vortex lines in Equation (158) is justified. Assuming that neutron superfluid is uniformly co-rotating with the star, the density of vortices per square kilometer is then given by $n_{v} \simeq 10^{14} / P$, where $P$ is the rotation period in seconds. With a rotation period of 33 milliseconds, a pulsar like the Crab is threaded by an array of about $10^{18}$ vortex lines (assuming a radius of $10 \mathrm{~km}$ )! Likewise, proton superconductors (assumed to be of type II [41]) in the core of neutron stars (and possibly in the pasta layers; see Section 3.3) is threaded by quantized magnetic flux tubes each carrying a magnetic field on the order of $10^{15} \mathrm{G}$. The surface density of flux tubes is about $10^{13}$ times that of neutron vortices. The interactions between the neutron vortices and the flux tubes are likely to affect the dynamic evolution of the star [354, 355].

\subsubsection{Dynamics of superfluid vortices}

In this section, we will discuss the nonrelativistic dynamics of superfluid vortices. The generalization to relativistic dynamics has been discussed in detail by Carter [71]. According to the Helmholtz theorem, the vortex lines are frozen in superfluid and move with the same velocity unless some force acts on them. The dynamics of a vortex line through the crust is governed by different types of forces, which depend on the velocities $\boldsymbol{v}_{\mathbf{f}}, \boldsymbol{v}_{\boldsymbol{v}}$ and $\boldsymbol{v}_{\mathbf{c}}$ of the bulk neutron superfluid, the vortex and the solid crust, respectively.

- A viscous drag force (not to be confused with entrainment, which is a nondissipative effect; see Section 8.3.6) opposes relative motion between a vortex line and the crust, inducing dissipation. At sufficiently small relative velocities, the force per unit length of the vortex line can be written as

$$
\mathcal{F}_{\mathbf{d}}=-\mathcal{R}\left(\boldsymbol{v}_{\boldsymbol{v}}-\boldsymbol{v}_{\mathbf{c}}\right)
$$

where $\mathcal{R}$ is a positive resistivity coefficient, which is determined by the interactions of the neutron vortex line with the nuclear lattice and the electron gas. The pinning of the vortex line to the crust is the limit of very strong drag entailing that $\boldsymbol{v}_{\boldsymbol{v}}=\boldsymbol{v}_{\mathbf{c}}$.

Living Reviews in Relativity

http://www. livingreviews.org/lrr-2008-10 
- Relative motion of a vortex line with respect to bulk superfluid (caused by drag or pinning) gives rise to a Magnus or lift force (analog to the Lorentz force), given by

$$
\mathcal{F}_{\mathbf{m}}=\rho_{\mathrm{f}} \boldsymbol{\kappa} \times\left(\boldsymbol{v}_{\mathbf{f}}-\boldsymbol{v}_{\boldsymbol{v}}\right),
$$

where $\rho_{\mathrm{f}}$ is the mass density of the free superfluid neutrons and $\boldsymbol{\kappa}$ is a vector oriented along the superfluid angular velocity and whose norm is given by $h / 2 m_{n}$ (see Carter \& Chamel [75] for the generalization to multi-fluid systems).

- A tension force resists the bending of the vortex line and is given by

$$
\mathcal{F}_{\mathbf{t}}=-\rho_{\mathrm{f}} \kappa \mathcal{C}_{\mathrm{t}} \frac{\partial^{2} \boldsymbol{u}}{\partial z^{2}},
$$

where $\boldsymbol{u}$ is the two-dimensional displacement vector of the vortex line directed along the $z$-axis. $\mathcal{C}_{\mathrm{t}}$ is a rigidity coefficient of order

$$
\mathcal{C}_{\mathrm{t}} \sim \frac{\kappa}{4 \pi} \ln \frac{d_{v}}{r_{v}},
$$

where $\kappa=h / 2 m_{n}, d_{v}$ is the intervortex spacing and $r_{v}$ the size of the vortex core [383].

All forces considered above are given per unit length of the vortex line. Let us remark that even in the fastest millisecond pulsars, the intervortex spacing (assuming a regular array) of order $d_{v} \sim$ $10^{-3}-10^{-4} \mathrm{~cm}$ is much larger than the size of the vortex core $r_{v} \sim 10-100$ fermis. Consequently the vortex-vortex interactions can be neglected.

The dynamic evolution of a vortex line is governed by

$$
\frac{m_{v}}{L_{v}} \frac{\mathrm{d} \boldsymbol{v}_{\boldsymbol{v}}}{\mathrm{d} t}=\mathcal{F}_{\mathbf{d}}+\mathcal{F}_{\mathbf{t}}+\mathcal{F}_{\mathbf{m}}
$$

where $m_{v}$ is the inertial mass of the vortex line and $L_{v}$ its length. Since the free neutron density inside the vortex core is typically much smaller than outside (unless the line is pinned to nuclei) [134, $433,27]$, the motion of the vortex line is accompanied by a rearrangement of the free neutrons. The inertial mass of the vortex line is approximately equal to the mass density of the neutron superfluid $\rho_{\mathrm{f}}$ times the volume of the line $\pi r_{v}^{2} L_{v}$ so that $m_{v} / L_{v} \sim \rho_{\mathrm{f}} \pi r_{v}^{2}$.

On a scale much larger than the intervortex spacing, the drag force $\mathcal{F}_{\mathbf{d}}$ acting on every vortex line leads to a mutual friction force between the neutron superfluid and the normal constituents. Assuming that the vortex lines are rigid and form a regular array, the mutual friction force, given by $\boldsymbol{f}_{\mathrm{d}}=n_{v} \mathcal{F}_{\mathrm{d}}$, can be obtained from Equation (173) after multiplying by the surface density $n_{v}$. Since $n_{v} \sim 1 / \pi d_{v}^{2}$, the inertial term on the left-hand side of Equation (173) is proportional to $\left(r_{v} / d_{v}\right)^{2} \ll 1$ and can be neglected. Solving the force balance equation yields the mutual friction force (per unit volume) [18]

$$
f_{\mathbf{d}}=n_{v} \mathcal{F}_{\mathbf{d}}=\frac{\mathcal{B}^{2}}{1+\mathcal{B}^{2}} \rho_{\mathrm{f}} n_{v} \boldsymbol{\kappa} \times\left(\boldsymbol{v}_{\mathbf{N}}-\boldsymbol{v}_{\mathbf{f}}\right)+\frac{\mathcal{B}}{1+\mathcal{B}^{2}} \rho_{\mathrm{f}} n_{v} \hat{\boldsymbol{\kappa}} \times \boldsymbol{\kappa} \times\left(\boldsymbol{v}_{\mathbf{c}}-\boldsymbol{v}_{\mathbf{f}}\right),
$$

where $n_{v}$ is the surface density of vortices in a plane perpendicular to the axis of rotation and $\mathcal{B}$ is a dimensionless parameter defined by

$$
\mathcal{B}=\frac{\mathcal{R}}{\rho_{\mathrm{f}} \kappa} .
$$

Different dissipative mechanisms giving rise to a mutual friction force have been invoked: scattering of electrons/lattice vibrations (phonons)/impurities/lattice defects by thermally excited neutrons in vortex cores [141, 189, 222], electron scattering off the electric field around a vortex line [48], 
and coupling between phonons and vortex line oscillations (Kelvin modes) [139, 223]. In the weak coupling limit $\mathcal{B} \rightarrow 0$, the vortices co-rotate with the bulk superfluid (Helmholtz theorem), while in the opposite limit $\mathcal{B} \rightarrow+\infty$, they are "pinned" to the crust. In between these two limits, in a frame co-rotating with the crust, the vortices move radially outward at angle $\operatorname{atan}(\mathcal{R})$ with respect to the azimuthal direction. The radial component of the vortex velocity reaches a maximum at $\mathcal{R}=1$.

Vortex pinning plays a central role in theories of pulsar glitches. The strength of the interaction between a small segment of the vortex line and a nucleus remains a controversial issue $[5,138,333$, 134, 120, 121, 122, 27]. The actual "pinning" of the vortex line (i.e., $\boldsymbol{v}_{\boldsymbol{v}}=\boldsymbol{v}_{\mathbf{c}}$ ) depends not only on the vortex-nucleus interaction, but also on the structure of the crust, on the rigidity of lines and on the vortex dynamics. For instance, assuming that the crust is a polycrystal, a rigid vortex line would not pin to the crust simply because the line cannot bend in order to pass through the nuclei, independent of the strength of the vortex-nucleus interaction! Recent observations of long-period precession in PSR 1828-11 [387], PSR B1642-03 [371] and RX J0720.4-3125 [180] suggest that, at least in those neutron stars, the neutron vortices cannot be pinned to the crust and must be very weakly dragged $[372,266]$.

Let us stress that the different forces acting on a vortex vary along the vortex line. As a consequence, the vortex lines may not be straight [198]. The extent to which the lines are distorted depends on the vortex dynamics. In particular, Greenstein [175] suggested a long time ago that vortex lines may twist and wrap about the rotation axis giving rise to a turbulent flow. This issue has been more recently addressed by several groups [323, 324, 288, 19]. In such a turbulent regime the mutual friction force takes the form [173]

$$
f_{\mathrm{d}}=\frac{\mathcal{C}_{\mathrm{d}}}{\kappa}\left(\boldsymbol{v}_{\mathbf{c}}-\boldsymbol{v}_{\mathbf{f}}\right)^{2}\left(\boldsymbol{v}_{\mathbf{c}}-\boldsymbol{v}_{\mathbf{f}}\right),
$$

where $\mathcal{C}_{\mathrm{d}}$ is a dimensionless temperature-dependent coefficient, assuming a dense random tangle of vortex lines.

\subsubsection{Superfluid hydrodynamics and entrainment}

One of the striking consequences of superfluidity is the allowance for several distinct dynamic components. In 1938, Tisza [404] introduced a two-fluid model in order to explain the properties of the newly discovered superfluid phase of liquid helium-4, which behaves either like a fluid with no viscosity in some experiments or like a classical fluid in other experiments. Guided by the Fritz London's idea that superfluidity is intimately related to Bose-Einstein condensation (which is now widely accepted), Tisza proposed that liquid helium is a mixture of two components, a superfluid component, which has no viscosity, and a normal component, which is viscous and conducts heat, thus, carrying all the entropy of the liquid. These two fluids are allowed to flow with different velocities. This model was subsequently developed by Landau[247, 246] and justified on a microscopic basis by several authors, especially Feynman [144]. Quite surprisingly, Landau never mentioned Bose-Einstein condensation in his work on superfluidity. According to Pitaevskii (as recently cited by Balibar [33]), Landau might have reasoned that superfluidity and superconductivity were similar phenomena (which is indeed true), incorrectly concluding that they could not depend on the Bose or Fermi statistics (see also the discussion by Feynman in Section 11.2 of his book [144]).

In Landau's two-fluid model, the normal part with particle density $n_{\mathcal{N}}$ and velocity $\boldsymbol{v}_{\mathcal{N}}$ is identified with the collective motions of the system or "quasiparticles" (see Section 8.3.1). The viscosity of the normal fluid is accounted for in terms of the interactions between those quasiparticles (see, for instance, the book by Khalatnikov [236] published in 1989 as a reprint of an original 1965 edition). Following the traditional notations, the superfluid component, with a particle density $n_{\mathcal{S}}$ and

Living Reviews in Relativity

http://www. livingreviews . org//rr-2008-10 
a "velocity" $\boldsymbol{v}_{\boldsymbol{s}}$, is locally irrotational except at singular points (see the discussion in Section 8.3.2)

$$
\boldsymbol{\nabla} \times \boldsymbol{v}_{\boldsymbol{s}}=0 .
$$

As pointed out many times by Brandon Carter, unlike $\boldsymbol{v}_{\mathcal{N}}$ the "superfluid velocity" is not a true velocity but is defined through

$$
\boldsymbol{p} \equiv m \boldsymbol{v}_{\boldsymbol{s}},
$$

where $\boldsymbol{p}$ is the true momentum per particle of the superfluid and $m$ is the mass of a helium atom. Although deeply anchored in the Lagrangian and Hamiltonian formulation of classical mechanics, the fundamental distinction between velocities and their canonical conjugates, namely momenta, has been traditionally obscured in the context of superfluidity. Note also that the superfluid density $n_{\mathcal{S}}$ coincides neither with the density $n$ of helium atoms (except at $T=0$ ) nor with the density $n_{\Psi}$ of atoms in the condensate ${ }^{10}$. The density of helium atoms is given by

$$
n=n_{\mathcal{S}}+n_{\mathcal{N}} .
$$

The confusion between velocity and momentum is very misleading and makes generalizations of the two-fluid model to multi-fluid systems (like the interior of neutron stars) unnecessarily difficult. Following the approach of Carter (see Section 10), the two-fluid model can be reformulated in terms of the real velocity $\boldsymbol{v}$ of the helium atoms instead of the superfluid "velocity" $\boldsymbol{v}_{\mathcal{S}}$. The normal fluid with velocity $\boldsymbol{v}_{\mathcal{N}}$ is then associated with the flow of entropy and the corresponding number density is given by the entropy density. At low temperatures, heat dissipation occurs via the emission of phonons and rotons. As discussed in Section 8.3.1, these quasiparticle excitations represent collective motions of atoms with no net mass transport (see, in particular, Figures 50 and 51). Therefore, the normal fluid does not carry any mass, i.e., its associated mass is equal to zero.

Following the general principles reviewed in Section 10, the momentum $\boldsymbol{p}$ of the superfluid helium atoms can be written as

$$
\boldsymbol{p}=m_{\star} \boldsymbol{v}+\left(m-m_{\star}\right) \boldsymbol{v}_{\mathcal{N}} .
$$

Note that the momentum $\boldsymbol{p}$ of the helium atoms is not simply equal to $m \boldsymbol{v}$ due to the scattering of atoms by quasiparticles. In the rest frame of the normal fluid, in which $v_{\mathcal{N}}=0$, the momentum and the velocity of the superfluid are aligned. However, the proportionality coefficient is not the (bare) atomic mass $m$ of helium but an effective mass $m_{\star}$. This effective mass $m_{\star}$ is related to the hydrodynamics of superfluid and should not be confused with the definitions employed in microscopic many-body theories. Before going further, let us remark that in the momentum rest frame of the normal component, the relation $\boldsymbol{p}=m \boldsymbol{v}$ holds! This can easily be shown from Equation (230), by using the identity (234) and remembering that the normal fluid is massless.

Comparing Equations (180) and (178) shows that the "superfluid velocity" in the original twofluid model of Landau is not equal to the velocity of the helium atoms but is a linear combination of both velocities $\boldsymbol{v}$ and $\boldsymbol{v}_{\mathcal{N}}$

$$
\boldsymbol{v}_{\mathcal{s}}=\frac{m_{\star}}{m} \boldsymbol{v}+\left(1-\frac{m_{\star}}{m}\right) \boldsymbol{v}_{\mathcal{N}} .
$$

The current of helium atoms is given by the sum of the normal and superfluid currents

$$
n \boldsymbol{v}=n_{\mathcal{S}} \boldsymbol{v}_{\mathcal{S}}+n_{\mathcal{N}} \boldsymbol{v}_{\mathcal{N}}
$$

Substituting Equation (181) into Equation (182) yields the relations

$$
n_{\mathcal{S}}=n \frac{m}{m_{\star}}, \quad n_{\mathcal{N}}=n\left(1-\frac{m}{m_{\star}}\right),
$$

\footnotetext{
${ }^{10}$ For instance at $T=0, n_{S}=n$ while $n_{\Psi} \simeq 0.1 n$
} 
which clearly satisfies $n=n_{\mathcal{S}}+n_{\mathcal{N}}$. The superfluid and normal densities can be directly measured in the experiment devised by Andronikashvili [21]. A stack of disks, immersed in superfluid, can undergo torsional oscillations about its axis. Due to viscosity, the normal component is dragged by motion of the disks, while the superfluid part remains at rest. The normal and superfluid densities can, thus, be obtained at any temperature by measuring the oscillation frequency of the disks. Since $n_{\mathcal{S}}(T) / n=m / m_{\star}(T)$, the dynamic effective mass $m_{\star}(T)$ can be determined experimentally. In particular, it is equal to the bare mass at $T=0, m_{\star}(T=0)=m$, and goes up as the temperature is raised, diverging at the critical point when superfluidity disappears. At any temperature $T>0$ the dynamic effective mass of an helium atom is therefore larger than the bare mass.

Entrainment effects, whereby momentum and velocity are not aligned, exist in any fluid mixtures owing to the microscopic interactions between the particles. But they are usually not observed in ordinary fluids due to the viscosity, which tends to equalize velocities. Even in superfluids like liquid Helium II, entrainment effects may be hindered at finite temperature ${ }^{11}$ by dissipative processes. For instance, when a superfluid is put into a rotating container, the presence of quantized vortices induces a mutual friction force between the normal and superfluid components (as discussed in Section 8.3.5). As a consequence, in the stationary limit the velocities of the two fluids become equal. Substituting $\boldsymbol{v}=\boldsymbol{v}_{\mathcal{N}}$ in Equation (180) implies that $\boldsymbol{p}=m \boldsymbol{v}$, as in the absence of entrainment.

\subsubsection{Entrainment effects in neutron stars}

A few years after the seminal work of Andreev \& Bashkin [20] on superfluid ${ }^{3} \mathrm{He}-{ }^{4} \mathrm{He}$ mixtures, it was realized that entrainment effects could play an important role in the dynamic evolution of neutron stars (see, for instance, [363] and references therein). For instance, these effects are very important for studying the oscillations of neutron star cores, composed of superfluid neutrons and superconducting protons [14]. Mutual entrainment not only affects the frequencies of the modes but, more surprisingly, (remembering that entrainment is a nondissipative effect) also affects their damping. Indeed, entrainment effects induce a flow of protons around each neutron superfluid vortex line. The outcome is that each vortex line carries a huge magnetic field $\sim 10^{14} \mathrm{G}$ [8]. The electron scattering off these magnetic fields leads to a mutual friction force between the neutron superfluid and the charged particles (see [18] and references therein). This mechanism, which is believed to be the main source of dissipation in the core of a neutron star, could also be at work in the bottom layers of the crust, where some protons might be unbound and superconducting (as discussed in Section 3.3).

It has recently been pointed out that entrainment effects are also important in the inner crust of neutron stars, where free neutrons coexist with a lattice of nuclear clusters [79, 78]. It is well known in solid state physics that free electrons in ordinary metals move as if their mass were replaced by a dynamic effective mass $m_{\star}^{e}$ (usually referred to as an optical mass in the literature) due to Bragg scattering by the crystal lattice (see, for instance, the book by Kittel [241]). The end result is that, in the rest frame of the solid, the electron momentum is given by

$$
\boldsymbol{p}^{\boldsymbol{e}}=m_{\star}^{e} \boldsymbol{v}_{\boldsymbol{e}},
$$

where $\boldsymbol{v}_{\boldsymbol{e}}$ is the electron velocity. This implies that in an arbitrary frame, where the solid (ion lattice) is moving with velocity $\boldsymbol{v}_{\mathbf{I}}$, the electron momentum is not aligned with the electron velocity but is given by

$$
\boldsymbol{p}^{\boldsymbol{e}}=m_{\star}^{e} \boldsymbol{v}_{\boldsymbol{e}}+\left(m_{e}-m_{\star}^{e}\right) \boldsymbol{v}_{\mathbf{I}},
$$

which is similar to Equation (180) for the momentum of superfluid Helium II. The concept of dynamic effective mass was introduced in the context of neutron diffraction experiments ten years

\footnotetext{
${ }^{11}$ Entrainment effects disappear as $T$ goes to zero since $m_{\star}(T=0)=m$ so that $\boldsymbol{p}=m \boldsymbol{v}$ according to Equation (180).
}

Living Reviews in Relativity

http://www. livingreviews.org//rr-2008-10 
ago [434] and has only recently been extended to the inner crust of neutron stars by Carter, Chamel \& Haensel [79, 78]. While the dynamic effective electron mass in ordinary metallic elements differs moderately from the bare mass $m_{\star}^{e} \sim 1-2 m_{e}$ (see, for instance, [204]), Chamel [90] has shown that the dynamic effective mass of free neutrons in neutron star crust could be very large, $m_{\star}^{\mathrm{f}} \sim 10-15 m_{n}$. The dynamics of the free neutrons is deeply affected by these entrainment effects, which have to be properly taken into account (see Section 10). Such effects are important for modeling various observed neutron star phenomena, like pulsar glitches (see Section 12.4) or neutron star oscillations (see Sections 12.5 and 12.6). 


\section{Conductivity and Viscosity}

\subsection{Introduction}

In this section, we will consider the transport of heat, electric charge, and momentum in neutron star crusts. In the absence of magnetic fields and assuming that the solid crust is isotropic ${ }^{12}$, the transport of heat and electric charge is described by the thermal conductivity $\kappa$ and the electrical conductivity $\sigma$ (Section 9.3) respectively. Under the same conditions, the transport of momentum is characterized by the shear viscosity $\eta$ and the bulk viscosity $\zeta$ (Section 9.4).

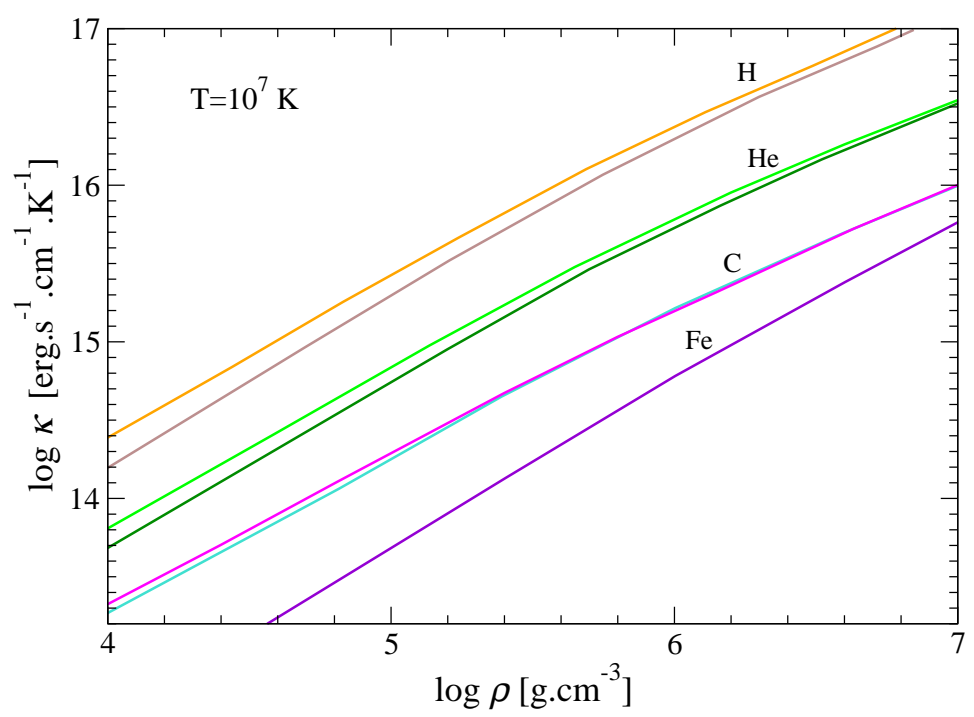

Figure 53: Thermal conductivity vs. mass density at $T=10^{7} \mathrm{~K}$ for four types of ions in the neutron star envelope. Lower curves: for each composition, electron-ion and electron-electron collisions included. Upper curves: electron-ion collisions only. Based on Figure 6 from [339].

Except for the very outer envelope, the main carriers in the transport processes in the outer crust are electrons, and they scatter mainly off ions (exceptions will be mentioned at the end of the corresponding sections). Theoretical techniques for the calculation of the transport coefficients in neutron star crusts are to a large extent borrowed from solid state physics, the classical reference still remaining the book of Ziman [435]. However, one has to remember that the density/temperature conditions within neutron star crusts are tremendously different from those in terrestrial solids, so that special care concerning the approximations used should be taken.

\subsection{Boltzmann equation for electrons and its solutions}

The electron distribution function is $f(\boldsymbol{p}, \boldsymbol{r}, t, s)$, where $\boldsymbol{p}$ and $\boldsymbol{r}$ are electron momentum and position vectors, respectively, $t$ is time, and $s$ is the electron spin projection on the spin quantization axis. The distribution function $f(\boldsymbol{p}, \boldsymbol{r}, t, s)$ satisfies the Boltzmann equation (BE) for electrons. At first glance, the validity of the BE (originally derived for a gas of particles) for a super dense plasma of electrons may seem paradoxical. However, electrons are strongly degenerate, so that only electron states in a thin shell around the chemical potential $\mu_{e}$ with energies $\left|\epsilon-\mu_{e}\right| \lesssim k_{\mathrm{B}} T$ are involved in the transport phenomena. In other words, the gas of "electron excitations" is dilute. Also, the

\footnotetext{
${ }^{12}$ This assumption may not remain valid in the "nuclear pasta" layers at the bottom of the crust discussed in Section 3.3
}

Living Reviews in Relativity

http://www . livingreviews . org//rr-2008-10 
infinite range of Coulomb interactions in vacuum is no longer a problem in dense electron-nuclear plasma because of screening. Moreover, as the kinetic Fermi energy of electrons is much larger than the Coulomb energy per electron, the Coulomb energy can be treated as a small perturbation and the electrons can be considered as a quasi-ideal Fermi gas.

We consider spin-unpolarized plasma. Then $f$ does not depend on $s$ and the BE for electrons reads

$$
\frac{\partial f}{\partial t}+\boldsymbol{v} \cdot \frac{\partial f}{\partial \boldsymbol{r}}+\boldsymbol{F} \cdot \frac{\partial f}{\partial \boldsymbol{p}}=I_{e}[f]
$$

where $\boldsymbol{v}$ is the electron velocity, and $\boldsymbol{F}$ is the external force acting on the electrons (e.g., electrostatic force $\boldsymbol{F}=-e \boldsymbol{E}$, where electron electric charge is $-e)$. The total collision integral $I_{e}[f]$, which is a functional of $f$, is a sum of collision integrals corresponding to collisions of electrons with nuclei, electrons, and impurities in the crystal lattice, $I_{e}[f]=I_{e \mathrm{~N}}[f]+I_{e e}[f]+I_{\mathrm{imp}}[f]$.

The additivity of partial collision integrals is valid when the scatterers are uncorrelated. This assumption may seem surprising for a crystal. The electron scatters off a lattice by exciting it, i.e., transferring energy and momentum to the lattice. This process of electron-lattice interactions corresponds to the creation and absorption of phonons, which are the elementary excitations of the crystal lattice. In this way the electron-lattice interaction is equivalent to the scattering of electrons by phonons. At temperatures well below the Debye temperature, $T<\frac{1}{4} \Theta$, the gas of phonons is dilute, and e-N scattering, represented by $I_{e N}[f]$, is actually the electron scattering by single phonons. These phonons form a Bose gas, and their number density and mean energy depend on $T$.

In the absence of external forces, the solution of Equation (186) is the Fermi-Dirac distribution function, $f^{(0)}$, corresponding to full thermodynamic equilibrium. The collision integrals then vanish, $I_{e j}\left[f^{(0)}\right]=0$, with $j=\mathrm{N}, e$, imp. We shall now show how to calculate the conductivities $\kappa$ and $\sigma$. Let us consider small stationary perturbations characterized by gradients of temperature $\boldsymbol{\nabla} T$, of the electron chemical potential $\nabla \mu_{e}$, and let us apply a weak constant electric field $\boldsymbol{E}$. The plasma will become slightly nonuniform, with weak charge and heat currents flowing through it. We assume that the length scale of this nonuniformity is much larger than the electron mean free path. Therefore, any plasma element will be close to a local thermodynamic equilibrium. However, gradients of $T$ and $\mu_{e}$, as well as $\boldsymbol{E}$, will induce a deviation of $f$ from $f^{(0)}$ and will produce heat and charge currents.

The next step consists in writing $f=f^{(0)}+\delta f$, where $\delta f$ is a small correction to $f^{(0)}$, linear in $\boldsymbol{\nabla} T, \nabla \mu_{e}$, and $\boldsymbol{E}$. We introduce the enthalpy per electron $h=\mu_{e}+\left(S_{e} / n_{e}\right) T$, where $S_{e}$ is the electron entropy density. The linearized left-hand side of Equation (186) is then

$$
-\left[\frac{\epsilon-h}{T} \nabla T+\boldsymbol{E}+\frac{\nabla \mu_{e}}{e}+\frac{S_{e}}{n_{e}} \frac{\nabla T}{e}\right] \cdot \boldsymbol{v} \frac{\partial f^{(0)}}{\partial \epsilon} .
$$

The general form of $\delta f$ is [435]

$$
\delta f=\Phi \frac{\partial f^{(0)}}{\partial \epsilon}=-\frac{\Phi}{k_{\mathrm{B}} T} f^{(0)}\left(1-f^{(0)}\right),
$$

where $\Phi$ is a slowly varying function of electron energy $\epsilon_{e}$. This specific form of $\delta f$ results from the $\mathrm{BE}$, and deserves a comment. In the limit of strong electron degeneracy $T \ll T_{\mathrm{Fe}}$ where $T_{\mathrm{F} e}$ is given by Equation (5), the derivative $\partial f^{(0)} / \partial \epsilon$ is strongly peaked at $\epsilon \approx \mu_{e}$. Actually, for $T / T_{\mathrm{Fe}} \longrightarrow 0$ we get $\partial f^{(0)} / \partial \epsilon \longrightarrow-\delta\left(\epsilon-\mu_{e}\right)$. On the contrary, $\Phi$ is a slowly varying function of $\epsilon$.

In our case, the general form of $\Phi$ linear in $\boldsymbol{\nabla} T, \boldsymbol{\nabla} \mu_{e}$ and $\boldsymbol{E}$ can be written as [435]

$$
\Phi=\frac{\epsilon-h}{T} A_{T}(\epsilon) \boldsymbol{v} \cdot \nabla T+e A_{e}(\epsilon) \boldsymbol{v} \cdot \boldsymbol{E}^{*}
$$


where

$$
\boldsymbol{E}^{*}=\boldsymbol{E}+\frac{\nabla \mu_{e}}{e}+\frac{S_{e}}{n_{e}} \frac{\nabla T}{e}
$$

and the coefficients $A_{e}(\epsilon)$ and $A_{T}(\epsilon)$ are functions of electron energy $\epsilon$. They fully determine $\kappa$ and $\sigma$. However, to determine them, we have to linearize the collision integrals with respect to $\Phi$, and then solve the linearized BE.

As the nuclei are very heavy compared to the electrons, the typical electron energy transferred during a collision is much smaller than $k_{\mathrm{B}} T$. The collision integral then takes the simple form (see, e.g., Ziman [435])

$$
I_{e \mathrm{~N}}[f] \approx-\frac{\delta f}{\tau_{0}(\epsilon)}
$$

This is the relaxation time approximation, and $\tau_{0}(\epsilon)$ is an effective relaxation time for the electron distribution function at an energy $\epsilon$. For strongly degenerate electrons we should put $\epsilon=\mu_{e}$ in the argument of $\tau_{0}(\epsilon)$.

This simple relaxation time approximation breaks down at $T \lesssim T_{\mathrm{pi}}$, when the quantum effects in the phonon gas become pronounced so that the typical energies transferred become $\sim k_{\mathrm{B}} T$ (and the number of phonons becomes exponentially small). The dominance of electron-phonon scattering breaks down at very low $T$. Simultaneously, $I_{e e}$ has a characteristic low- $T \ll T_{\mathrm{F} e}$ behavior $I_{e e} \propto\left(T / T_{\mathrm{Fe}}\right)^{2}$. All this implies the dominance of the e-impurity scattering in the low- $T$ limit, $I_{\mathrm{imp}} \gg I_{e e}, I_{e \mathrm{~N}}$.

The scattering of electrons on ions (nuclei) can be calculated from the Coulomb interaction, including medium effects (screening). An effective scattering frequency of an electron of energy $\epsilon$ , denoted $\nu_{e N}(\epsilon)$, is related to the corresponding transport scattering cross section $\sigma_{\operatorname{tr}}(\epsilon)$ by

$$
\nu_{e \mathrm{~N}}(\epsilon)=\frac{1}{\tau_{0}(\epsilon)}=n_{\mathrm{N}} v \sigma_{\mathrm{tr}}(\epsilon)
$$

where $v=\partial \epsilon / \partial p$ is electron velocity. The transport scattering cross section is calculated by the integration of the differential scattering cross section,

$$
\sigma_{\operatorname{tr}}(\epsilon)=2 \pi \int_{0}^{\pi} \mathrm{d} \vartheta \sin \vartheta \sigma(\epsilon, \vartheta)(1-\cos \vartheta),
$$

where $\vartheta$ is the electron scattering angle.

The electron-nucleus scattering is quasi-elastic at $T>T_{\mathrm{pi}}$, with electron energy change $\delta \epsilon \ll$ $k_{\mathrm{B}} T$. The function $\sigma_{\mathrm{tr}}(\epsilon)$ can be calculated, including screening and relativistic effects. After scattering, the electron momentum changes by $\hbar q$ within $q_{\min } \leq q \leq q_{\max }$. Therefore, the formula for $\sigma_{\operatorname{tr}}(\epsilon)$ can be rewritten as

$$
\sigma_{\operatorname{tr}}(\epsilon)=4 \pi \frac{Z^{2} e^{4}}{p^{2} v^{2}} \Lambda_{e \mathrm{~N}}(\epsilon)
$$

where $\Lambda_{e N}(\epsilon)$ is the famous Coulomb logarithm of the plasma transport theory. The Coulomb logarithm is directly related to the Fourier transform of the complete electron-nucleus interaction, $\phi_{q}$, by

$$
\Lambda_{e \mathrm{~N}}(\epsilon)=\int_{q_{\min }}^{q_{\max }} \mathrm{d} q q^{3}\left|\phi_{q}\right|^{2}
$$

where $q_{\min }$ is related to the screening and $q_{\max }=2 p_{\mathrm{F}} / \hbar$ for strongly degenerate electrons. In a concise numerical form

$$
\tau_{e \mathrm{~N}}=\frac{1}{\nu_{e \mathrm{~N}}\left(\mu_{e}\right)}=\frac{5.70 \times 10^{-17} \mathrm{~s}}{\gamma_{\mathrm{r}} Z \Lambda_{e \mathrm{~N}}},
$$


where $\gamma_{\mathrm{r}}$ is given by Equation (6). In the relaxation time approximation the calculation of transport coefficients reduces to the calculation of the Coulomb logarithm.

A second important approximation (after the relaxation time one) is expressed as the Matthiessen rule. In reality, the electrons scatter not only off nuclei $(e \mathrm{~N})$, but also by themselves $(e e)$, and off randomly distributed impurities (imp), if there are any. The Matthiessen rule (valid under strong degeneracy of electrons) states that the total effective scattering frequency is the sum of frequencies on each of the scatterers.

For heat conduction the Matthiessen rule gives, for the total effective scattering frequency of electrons,

$$
\nu_{\kappa}=\nu_{e \mathrm{~N}}^{\kappa}+\nu_{e e}^{\kappa}+\nu_{\mathrm{imp}}^{\kappa} .
$$

Notice that, as ee scattering does not change the electric current, it will not contribute to electrical conductivity and to $\nu_{\sigma}$, so that the Matthiessen rule gives

$$
\nu_{\sigma}=\nu_{e \mathrm{~N}}^{\sigma}+\nu_{\mathrm{imp}}^{\sigma}
$$

Electron scattering on randomly distributed impurities in some lattice sites is similar to the

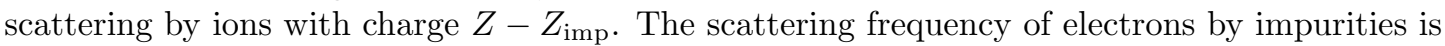

$$
\nu_{\mathrm{imp}}=\frac{4 \pi e^{4}}{p_{\mathrm{F} e}^{2} v_{\mathrm{F} e}} \sum_{\mathrm{imp}}\left(Z-Z_{\mathrm{imp}}\right)^{2} n_{\mathrm{imp}} \Lambda_{\text {eimp }},
$$

where $n_{\mathrm{imp}}$ is the number density of impurities of a given type "imp" and the sum is over all types "imp". Detailed calculations of $\nu_{e \mathrm{~N}}^{\kappa}$ and $\nu_{e \mathrm{~N}}^{\sigma}$ in liquid and solid plasma of neutron star envelopes, taking into account additional effects, such as electron-band structures and multi-phonon processes, are presented in [339]. There one can find analytic fitting formulae, which are useful for applications.

Recently, the calculation of $\nu_{e e}^{\kappa}$ has been revised, taking into account the Landau damping of transverse plasmons [378]. This effect strongly reduces $\nu_{e e}^{\kappa}$ for ultrarelativistic electrons at $T<T_{\mathrm{p} e}$.

In the presence of a magnetic field $\boldsymbol{B}$, transport properties become anisotropic, as briefly described in Section 9.5.

\subsection{Thermal and electrical conductivities}

A small temperature gradient $\nabla T$ and a weak constant electric field $\boldsymbol{E}$ induce a heat current $\boldsymbol{j}_{T}$ and an electric current $\boldsymbol{j}_{e}$

$$
\begin{gathered}
\boldsymbol{j}_{T}=-Q_{T} T \boldsymbol{j}_{e}-\kappa \boldsymbol{\nabla} T \\
\boldsymbol{j}_{e}=\sigma \boldsymbol{E}^{*}+\sigma Q_{T} \boldsymbol{\nabla} T
\end{gathered}
$$

where $Q_{T}$ is the thermopower, $\sigma$ is electrical conductivity, and $\kappa$ is thermal conductivity. Here, $\boldsymbol{E}^{*}$ is an "effective electric field", defined in Equation (190).

Both conductivities can be expressed in terms of the corresponding effective scattering frequency, calculated in the preceding section as

$$
\begin{gathered}
\sigma=\frac{e^{2} n_{e}}{m_{e}^{*} \nu_{\sigma}}, \\
\kappa=\frac{\pi^{2} k_{\mathrm{B}}^{2} T n_{e}}{3 m_{e}^{*} \nu_{\kappa}},
\end{gathered}
$$

where the electron effective mass is given by Equation (1). 
In the relaxation time approximation and for strongly degenerate electrons we get

$$
\nu_{\kappa}=\nu_{\sigma} \simeq \nu_{e N}\left(\mu_{e}\right)
$$

so that the Wiedemann-Franz law is satisfied:

$$
\kappa=\frac{\pi^{2} k_{\mathrm{B}}^{2} T}{3 e^{2}} \sigma
$$

Let us remind ourselves, that the equality (204) is violated when the ee scattering is not negligible compared to electron scattering by nuclei and by impurities. This and other effects leading to violation of the Wiedemann-Franz law are discussed in [339].
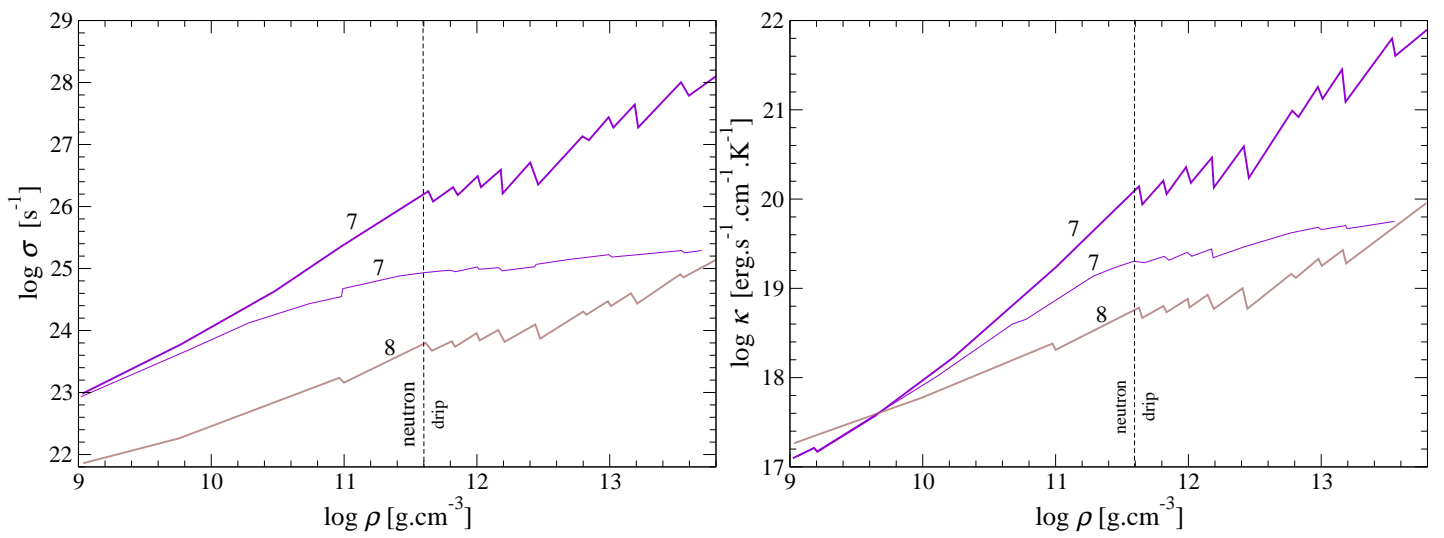

Figure 54: Electrical conductivity $\sigma$ and thermal conductivity $\kappa$ of the outer and inner crust, calculated for the ground-state model of Negele \& Vautherin [303]. Labels 7 and 8 refer to $\log _{10} T[\mathrm{~K}]=7$ and 8 , respectively. The thin line with label 7 corresponds to an impure crust, which contains in the lattice sites $5 \%$ impurities - nuclei with $\left|Z_{\text {imp }}-Z\right|=4$. Based on a figure made by A.Y. Potekhin.
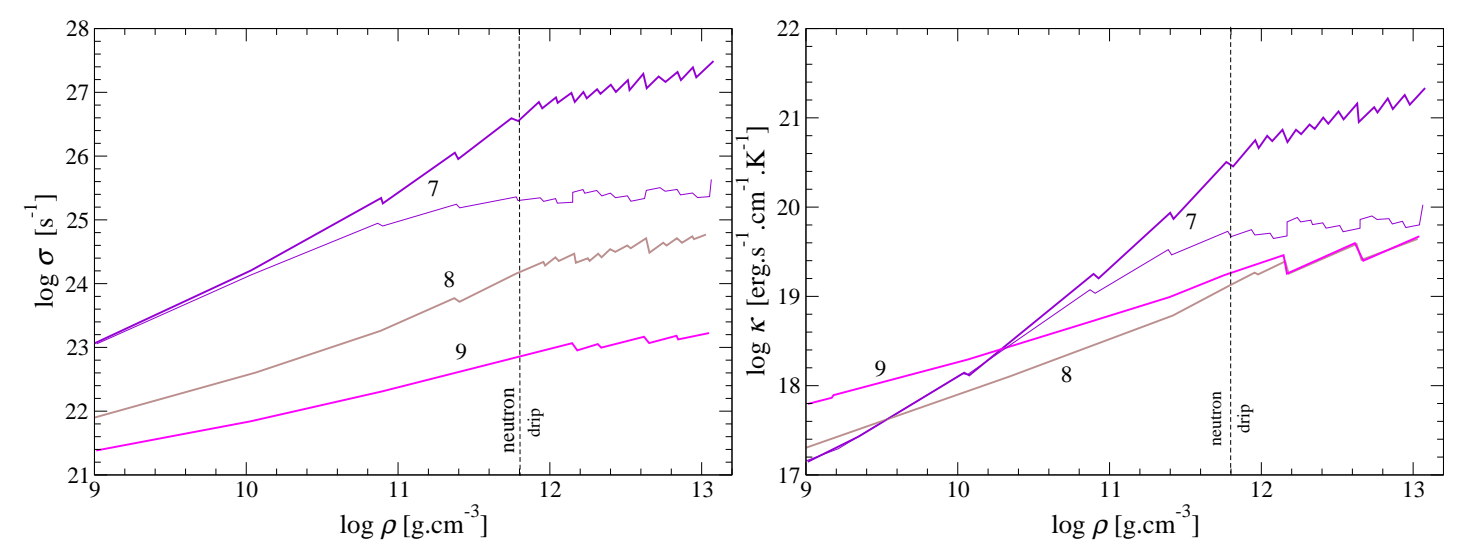

Figure 55: The same as in Figure 54 but calculated for the accreted-crust model of Haensel \& Zdunik [185]. Results obtained assuming $5 \%$ of nuclei - impurities with $\left|Z_{\text {imp }}-Z\right|=2$. Based on a figure made by A.Y. Potekhin.

The role of neutron gas in the inner crust deserves comment. Its normal component contributes to $\kappa$, so that

$$
\kappa=\kappa_{e}+\kappa_{n}
$$


However, there are actually two contributions to $\kappa_{n}$. The first one results from the scattering and is therefore of a standard "diffusive" nature. This contribution to $\kappa_{n}$ is

$$
\kappa_{n}^{\mathrm{diff}}=\frac{\pi^{2} k_{\mathrm{B}}^{2} T n_{n}}{3 m_{n}^{*} \nu_{n}},
$$

where $n_{n}$ is the number density of the gas of "neutron excitations" (neutrons are strongly degenerate and moreover superfluid), $m_{n}^{*}$ is their effective mass, and $\nu_{n}$ is their scattering frequency. Neutron excitations scatter by nuclear clusters and by themselves via strong interactions,

$$
\nu_{n}=\nu_{n \mathrm{~N}}+\nu_{n n} .
$$

However, due to a much smaller neutron-neutron cross section as compared to the neutron-cluster one, and due to the low density of neutron excitations, we get $\nu_{n \mathrm{~N}} \gg \nu_{n n}$, so that $\nu_{n} \approx \nu_{n \mathrm{~N}}$. The second contribution to $\kappa_{n}$ is characteristic of superfluids and has a convective character ("convective counterflow", see, e.g., Tilley \& Tilley [403]); we denote it by $\kappa_{n}^{\text {conv }}$.

It can be noted that for $T<T_{\mathrm{m}}$ "neutron excitations" scatter by the lattice phonons. Complete calculation of $\kappa_{n}$, taking due account of the effect of the crystal lattice on neutron scattering and neutron superfluidity remains to be done.

The presence of impurities considerably decreases electrical and thermal conductivities at low temperature and high density; see Figure 54 . At $T=10^{7} \mathrm{~K}, 5 \%$ of impurities with $\left|Z_{\text {imp }}-Z\right|=4$ reduces $\sigma$ and $\kappa$ at $\rho=10^{13} \mathrm{~g} \mathrm{~cm}^{-3}$ by two orders of magnitude. Accreted crusts are characterized by nuclides with lower values of $A$ and $Z$ than those in the ground-state crust. Accordingly, accreted crusts have higher electrical and thermal conductivities than the ground-state crust of the same $\rho$ and T. This is illustrated in Figure 55. Notice the differences between the $\sigma$ and $\kappa$ plots at $10^{8} \mathrm{~K}$ and $10^{9} \mathrm{~K}$. They are due to an additional factor $T$ in $\kappa$, reflected in the Wiedermann-Franz law (205).

Recent calculations of $\kappa_{e e}$, taking into account the Landau damping of transverse plasmons, give a much larger contribution from ee scattering than the previous ones, using the static screening, on which Figures 54 and 55 are based. As shown by Shternin and Yakovlev [378], the Landau damping of transverse plasmons strongly reduces $\nu_{e}^{\kappa}$ in the inner crust at $T \lesssim 10^{7} \mathrm{~K}$.

The contribution of ions to $\kappa$ was recently calculated by Chugunov and Haensel [100], who also quote older papers on this subject. As a rule, $\kappa_{\mathrm{N}}$ can be neglected compared to $\kappa_{e}$. A notable exception, relevant for magnetized neutron stars, is discussed in Section 9.5.

\subsection{Viscosity}

In this section we consider the viscosity of the crust, which can be in a liquid or a solid phase. For strongly nonideal $(\Gamma \gg 1)$ and solid plasma the transport is mediated mainly by electrons. We will limit ourselves to this case only. For solid crust, we will assume a polycrystal structure, so that on a macroscopic scale the crust will behave as an isotropic medium.

Let us denote a stationary macroscopic hydrodynamic velocity field, imposed on the plasma, by $\boldsymbol{U}(\boldsymbol{r})$. In an isotropic plasma the viscous part of the stress tensor can be written as

$$
\Pi_{i j}^{\mathrm{vis}}=\eta\left(\frac{\partial U_{i}}{\partial x_{j}}+\frac{\partial U_{j}}{\partial x_{i}}-\frac{2}{3} \delta_{i j} \boldsymbol{\nabla} \cdot \boldsymbol{U}\right)+\zeta \delta_{i j} \boldsymbol{\nabla} \cdot \boldsymbol{U}
$$

where $\eta$ is the shear viscosity and $\zeta$ is the bulk viscosity. The viscous component of the stress tensor enters the equations of neutron star hydrodynamics and is relevant for neutron star pulsations.

First, let us consider volume preserving flows, characterized by $\boldsymbol{\nabla} \cdot \boldsymbol{U}=0$. A schematic view of such a flow in the solid crust, characteristic of torsional oscillations of the crust, is shown in Figure 58. The dissipation resulting in the entropy production is determined by the shear viscosity 


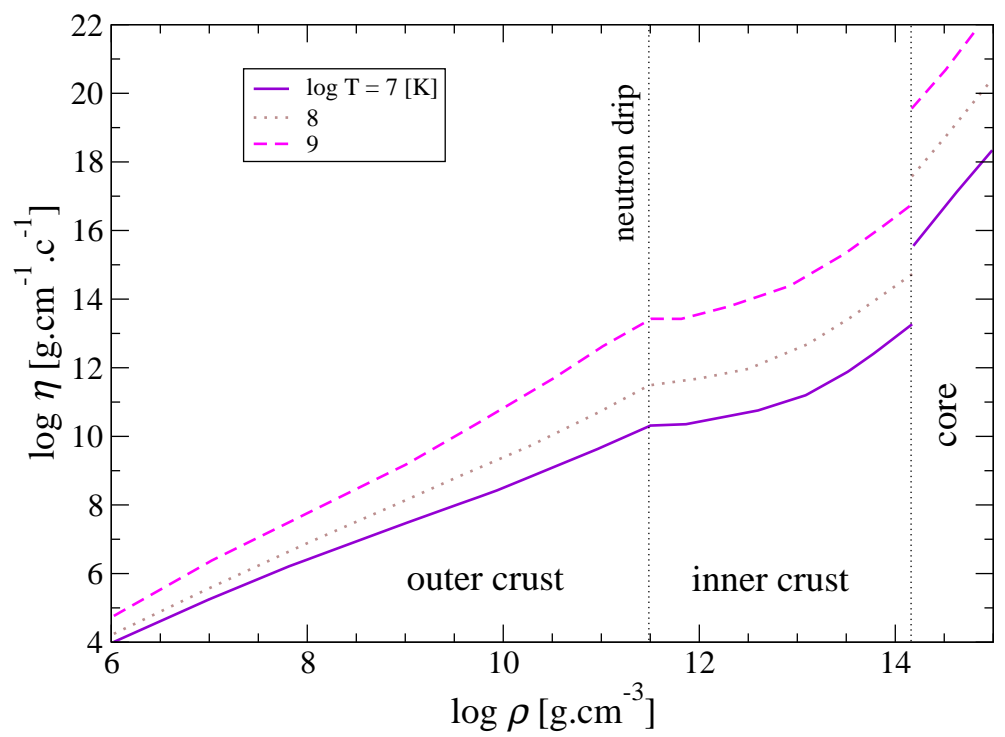

Figure 56: Electron shear viscosity of the crust and the upper layer of the core for $\log _{10} T[\mathrm{~K}]=7,8,9$. Calculated by Chugunov \& Yakovlev [101] with a smooth composition model of the ground-state (Appendix B of Haensel, Potekhin, and Yakovlev [184]).

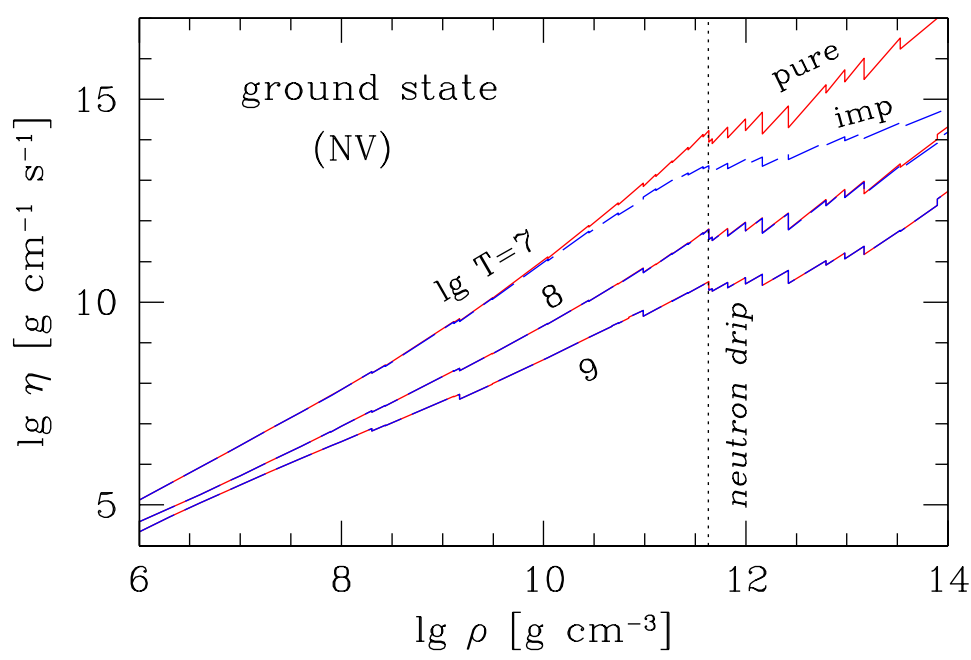

Figure 57: Electron contribution to crust viscosity and effect of impurities. Solid lines - perfect onecomponent plasma. Dashed line - admixture of impurities with $n_{\mathrm{imp}}=0.05 n_{\mathrm{i}}$ and $\left|Z-Z_{\mathrm{imp}}\right|=2$. Curves are labeled by $\log _{10} T[\mathrm{~K}]$. Impurity contribution to $\eta_{e}$ becomes dominant for $T \ll T_{\mathrm{pi}}$ : this is visible for a $\log _{10} T[\mathrm{~K}]=7$ curve. Ground-state crust model of Negele \& Vautherin [303] is used. Figure made by A.Y. Potekhin. 
$\eta$. In the outer crust, $\eta$ is a sum of the electron and nuclei contributions, $\eta=\eta_{e}+\eta_{\mathrm{N}}$, but for $\rho>10^{5} \mathrm{~g} \mathrm{~cm}^{-3} \eta_{e} \gg \eta_{\mathrm{N}}$ and $\eta \approx \eta_{e} \cdot{ }^{13}$. In the inner crust, an additional contribution from the normal component of the gas of dripped neutrons should be added.

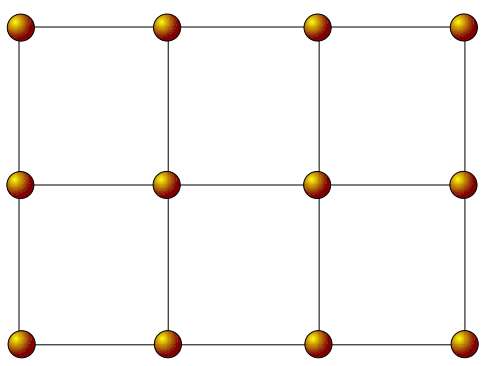

equilibrium

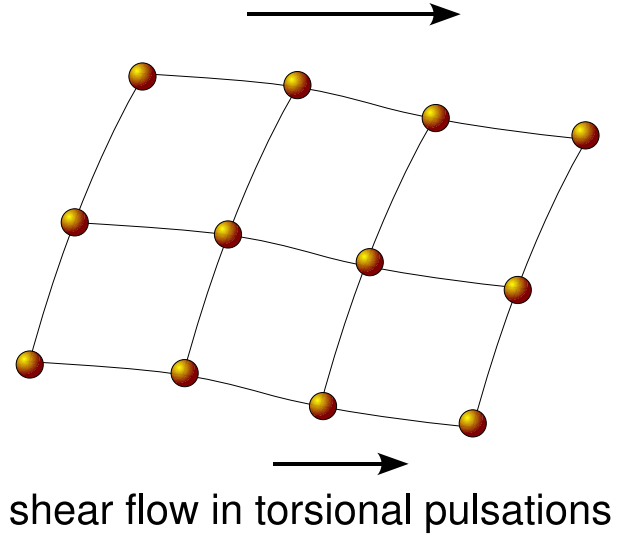

Figure 58: Schematic picture of torsional oscillations in neutron star crust. Left: equilibrium structure of the solid crust, represented as a two-dimensional square lattice. Right: shear flow in the crust (the shear velocity is indicated by arrows).

The electrons are scattered on nuclei, on impurity nuclei, and on themselves, so that the effective frequency of their scattering is given by the sum $\nu_{e}^{\eta}=\nu_{e \mathrm{~N}}^{\eta}+\nu_{\mathrm{imp}}^{\eta}+\nu_{e e}^{\eta}$. However, as long as the temperature is not too low, the approximation $\nu_{e} \approx \nu_{e N}$ can be used.

To calculate $\eta_{e}$ from the BE for electrons, we have to determine $\delta f=f-f^{(0)}$ due to the presence of a weak plasma velocity field, $\boldsymbol{U}$. The solution of the BE, linearized in $\boldsymbol{U}$ and in $\delta f$, has the form

$$
\delta f=A_{\eta}(\epsilon)[\boldsymbol{v} \cdot \nabla(\boldsymbol{p} \cdot \boldsymbol{U})] \frac{\partial f^{(0)}}{\partial \epsilon},
$$

where $A_{\eta}(\epsilon)$ is a function to be determined from the BE. In the relaxation time approximation and for strongly degenerate electrons $A_{\eta}=\tau_{e \mathrm{~N}}^{\eta}=1 / \nu_{e \mathrm{~N}}$ is the effective relaxation time due to $e \mathrm{~N}$ collisions, calculated at the electron Fermi surface.

The scattering frequency $\nu_{e N}$, in turn, can be expressed in terms of the effective Coulomb logarithm $\Lambda_{e \mathrm{~N}}$ by

$$
\nu_{e \mathrm{~N}}^{\eta}=12 \pi \frac{Z^{2} e^{4} n_{\mathrm{N}}}{p_{\mathrm{F} e}^{2} v_{\mathrm{F} e}} \Lambda_{e \mathrm{~N}} .
$$

Having calculated the Coulomb logarithm, we get the electron viscosity using a standard formula

$$
\eta_{e}=\frac{n_{e} p_{\mathrm{Fe}} v_{\mathrm{F} e}}{5 \nu_{e \mathrm{~N}}}
$$

At low temperature, impurities can become the main scatterers of electrons, with

$$
\nu_{\mathrm{imp}}=\frac{12 \pi e^{4}}{p_{\mathrm{F} e}^{2} v_{\mathrm{F} e}} \sum_{\mathrm{imp}}\left(Z-Z_{\mathrm{imp}}\right)^{2} n_{\mathrm{imp}} \Lambda_{e \mathrm{imp}} .
$$

In the inner crust, one must also consider the gas of dripped neutrons.

\footnotetext{
${ }^{13}$ Ion contribution to $\eta$ can be important in the very outer layers with $\rho<10^{4} \mathrm{~g} \mathrm{~cm}^{-3}$, where $\eta \approx \eta_{\mathrm{N}}$
} 
Calculations of the shear viscosity for the liquid phase were done by Flowers \& Itoh [146, 147] and Nandkumar \& Pethick [300]. Recently, calculations of the shear viscosity of the neutron star crust were done for both the liquid and the crystal phases, by Chugunov \& Yakovlev [101]; their results are displayed in Figure 56. These authors also give analytic fitting formulae for the effective Coulomb logarithms, which can be used for different models of the crust. The electron-impurity scattering becomes dominant at low $T$, when electron-lattice scattering (via phonons) is suppressed by quantum effects. This is visualized in Figure 57. Recently, the contribution to $\eta_{e}$ resulting from the ee scattering, was recalculated by Shternin [377], who took into account the Landau damping of transverse plasmons. The Landau damping of transverse plasmons leads to a significant suppression of $\eta_{e e}$ for ultra-relativistic electrons, and modifies the temperature dependence of $\eta_{e e}$.

We are not aware of any calculations of the bulk viscosity of the crust, $\zeta$. We just mention that it is generally assumed that the bulk viscosity of the crust is much smaller than the shear one, $\zeta \ll \eta$.

\subsection{Transport in the presence of strong magnetic fields}

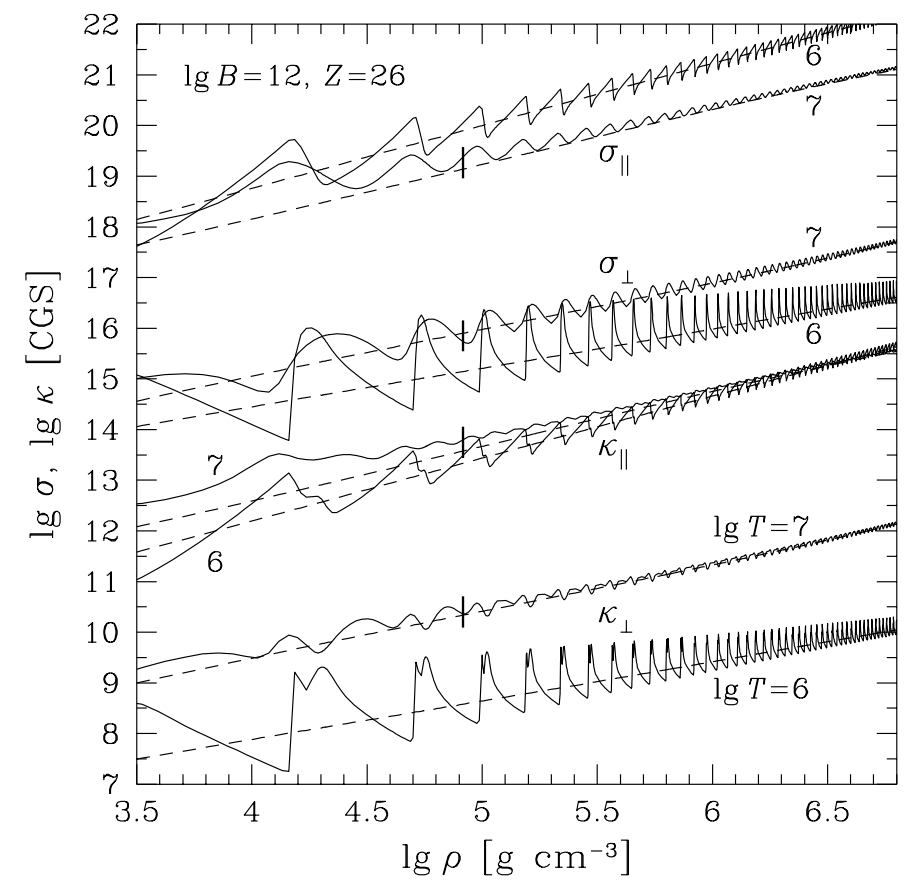

Figure 59: Longitudinal $(\|)$ and transverse $(\perp)$ electrical and thermal conductivities in the outer envelope composed of ${ }^{56} \mathrm{Fe}$ for $B=10^{12} \mathrm{G}$ and $\log _{10} T[\mathrm{~K}]=6,7$. Quantum calculations (solid lines) are compared with classical ones (dash lines). Vertical bars: liquid-solid transition at $T=10^{7} \mathrm{~K}$. Based on Figure 5 from [338].

We consider a surface magnetic field to be strong if $B \gg 10^{9} \mathrm{G}$. Such magnetic fields affect the accretion of plasma onto the neutron star and modify the properties of atoms in the atmosphere. On the contrary, a magnetic field $B \lesssim 10^{9} \mathrm{G}$, such as associated with millisecond pulsars or with most of the X-ray bursters, is considered to be weak. Typical pulsars are magnetized neutron 
stars, with the value of $\boldsymbol{B}$ near the magnetic pole $\sim 10^{12} \mathrm{G}$. Much stronger magnetic fields are associated with magnetars, $\sim 10^{14}-10^{15} \mathrm{G}$; such magnetic fields with $B \gtrsim 10^{14} \mathrm{G}$ are often called "super-strong". These magnetic fields can strongly affect transport processes within neutron star envelopes. Electron transport processes in magnetized neutron star envelopes and crusts are reviewed in $[338,412]$. In the present section we limit ourselves to a very brief overview.

Locally, a magnetic field can be considered uniform. We will choose the $z$ axis of a coordinate system along $\boldsymbol{B}$, so that $\boldsymbol{B}=[0,0, B]$. We will limit ourselves to the case of strongly degenerate electrons and we will assume the validity of the relaxation time approximation. Let relaxation time for $B=0$ be $\tau_{0}$. An important timescale associated with magnetic fields is the electron gyromagnetic frequency ${ }^{14}$

$$
\omega_{B}=\frac{e B}{m_{e}^{*} c} .
$$

The magnetic field bends electron trajectories in the $(x, y)$ plane, and suppresses the electron transport across $\boldsymbol{B}$. Therefore, the transport properties become anisotropic, and we must consider tensors $\kappa_{i j}$ and $\sigma_{i j}$, which can be written as

$$
\begin{gathered}
\kappa_{i j}=\frac{\pi^{2} k_{\mathrm{B}}^{2} T n_{e}}{3 m_{e}^{*}} \xi_{i j}^{\kappa}, \\
\sigma_{i j}=\frac{e^{2} n_{e}}{m_{e}^{*}} \xi_{i j}^{\sigma} .
\end{gathered}
$$

In what follows we will consider three basic regimes of transport in magnetic fields.

\subsubsection{Nonquantizing magnetic fields}

Many Landau orbitals are populated and quantum effects are smeared by thermal effects because $k_{\mathrm{B}} T>\hbar \omega_{\mathrm{ce}}$. Transport along the magnetic field is not affected by $\boldsymbol{B}$, while transport across $\boldsymbol{B}$ is fully described by the Hall magnetization parameters $\omega_{B} \tau_{0}^{\kappa, \sigma}$,

$$
\omega_{B} \tau_{0}^{\kappa, \sigma} \approx 1760 \frac{B_{12}}{\gamma_{\mathrm{r}}} \frac{\tau_{0}^{\kappa, \sigma}}{10^{-16} \mathrm{~s}}
$$

where $\tau_{0}^{\kappa, \sigma}$ are the effective relaxation times at $\boldsymbol{B}=0$ for the thermal and electric conductivities, respectively. The nonzero components of the $\xi_{i j}^{\kappa, \sigma}$ tensors are

$$
\xi_{z z}=\tau_{0}^{\kappa, \sigma}, \xi_{x x}^{\kappa, \sigma}=\xi_{y y}^{\kappa, \sigma}=\frac{\tau_{0}^{\kappa, \sigma}}{1+\left(\omega_{B} \tau_{0}^{\kappa, \sigma}\right)^{2}}, \xi_{x y}^{\kappa, \sigma}=\xi_{y x}^{\kappa, \sigma}=\frac{\omega_{B}\left(\tau_{0}^{\kappa, \sigma}\right)^{2}}{1+\left(\omega_{B} \tau_{0}^{\kappa, \sigma}\right)^{2}} .
$$

As an example, consider strongly degenerate electrons and dominating $e \mathrm{~N}$ scattering, $\tau_{0} \approx \tau_{e \mathrm{~N}}$. Then, Equation (196) yields $\omega_{B} \tau_{0}^{\kappa, \sigma} \approx 1003 B_{12} /\left(\gamma_{\mathrm{r}}^{2} Z \Lambda_{e \mathrm{~N}}\right)$.

\subsubsection{Weakly-quantizing magnetic fields}

Many Landau levels are populated by electrons, but quantization effects are well pronounced because $k_{\mathrm{B}} T<\hbar \omega_{\mathrm{ce}}$. There are two relaxation times, $\tau_{\|}^{\kappa, \sigma}$ and $\tau_{\perp}^{\kappa, \sigma}$, which oscillate with density (see below). As shown by Potekhin [338], the formulae for the nonzero components of the $\xi_{i j}^{\kappa, \sigma}$ tensors can be written in a form similar to Equation (218):

$$
\xi_{z z}^{\kappa, \sigma}=\tau_{\|}^{\kappa, \sigma}, \xi_{x x}^{\kappa, \sigma}=\xi_{y y}^{\kappa, \sigma}=\frac{\tau_{\perp}^{\kappa, \sigma}}{1+\left(\omega_{B} \tau_{\perp}^{\kappa, \sigma}\right)^{2}}, \xi_{x y}^{\kappa, \sigma}=\xi_{y x}^{\kappa, \sigma}=\frac{\omega_{B}\left(\tau_{\perp}^{\kappa, \sigma}\right)^{2}}{1+\left(\omega_{B} \tau_{\perp}^{\kappa, \sigma}\right)^{2}} .
$$

\footnotetext{
${ }^{14}$ The gyromagnetic frequency of electrons should not be confused with the electron cyclotron frequency $\omega_{\mathrm{c} e}=$ $e B / m_{e} c$ entering the formula for the energies of the Landau levels; see Section 2.
} 
At a fixed temperature and in the presence of a weakly quantizing $\boldsymbol{B}$, the density dependence of the components of the $\sigma$ and $\kappa$ tensors exhibits characteristic oscillations around the nonquantized (classical) values. Each oscillation corresponds to the filling of a new Landau level. The amplitude of these oscillations decreases with decreasing density. An example of the density dependence of $\sigma_{\|} \equiv \sigma_{z z}$ and $\sigma_{\perp} \equiv \sigma_{x x}=\sigma_{y y}$, and of the same components of the $\kappa_{i j}$ tensor, is presented in Figure 59. As we see in Figure 59, the "density period" of oscillation decreases with increasing $\rho$, and the oscillation amplitude decreases with increasing $T$. At $T=10^{7} \mathrm{~K}$, a magnetic field of $10^{12} \mathrm{G}$ is weakly quantizing at $\rho>10^{4.2} \mathrm{~g} \mathrm{~cm}^{-3}$.

\subsubsection{Strongly-quantizing magnetic fields}

Not only is $k_{\mathrm{B}} T<\hbar \omega_{\mathrm{ce}}$, but also most of the electrons are populating the ground Landau level. Both the values of $\sigma_{i j}$ and $\kappa_{i j}$ and their density dependence are dramatically different from those of the nonquantizing (classical) case. As shown by Potekhin [338], the formulae for $\sigma_{i j}$ and $\kappa_{i j}$ are still given by Equations (219). Analytical fitting formulae for $\tau_{\|}^{\kappa, \sigma}$ and $\tau_{\perp}^{\kappa, \sigma}$ are given in [338]. As seen in Figure 59 , at $T=10^{6} \mathrm{~K}$ a field of $10^{12} \mathrm{G}$ is strongly quantizing for $\rho<10^{4.2} \mathrm{~g} \mathrm{~cm}^{-3}$

\subsubsection{Possible dominance of ion conduction}

Thermal conduction by ions is much smaller than that by electrons along $\boldsymbol{B}$. However, the electron conduction across $\boldsymbol{B}$ is strongly suppressed. In outer neutron star crust, heat flow across $\boldsymbol{B}$ can be dominated by ion/phonon conduction[100]. This is important for the heat conduction across $\boldsymbol{B}$ in cooling magnetized neutron stars. Correct inclusion of the ion heat conductivity then leads to a significant reduction of the thermal anisotropy in the envelopes of magnetized neutron stars[100]. 


\section{Macroscopic Model of Neutron Star Crusts}

The understanding of many observed phenomena occurring in neutron stars (and briefly reviewed in Section 12, for instance, pulsar glitches or torsional oscillations in Soft Gamma Repeaters) requires modeling the dynamic evolution of the crust. So far theoretical efforts have been mainly devoted to modeling the dynamic evolution of the liquid core by considering a mixture of superfluid neutrons and superconducting protons (see, for instance, the recent review by Andersson \& Comer [15]).

Macroscopic models of neutron star crusts, taking into account the presence of the neutron superfluid at $\rho>\rho_{\mathrm{ND}}$ (see Section 8), have been developed by Carter and collaborators. They have shown how to extend the two-fluid picture of neutron star cores [93] to the inner crust layers in the Newtonian framework [79, 94]. They have also discussed how to calculate the microscopic coefficients of this model $[78,77]$. More elaborate models treating the crust as a neutron superfluid in an elastic medium and taking into account the effects of a frozen-in magnetic field have been very recently developed both in general relativity [73, 85] and in the Newtonian limit [73, 72]. All these models are based on an action principle that will be briefly reviewed in Section 10.1. We will consider a simple nonrelativistic two-fluid model of neutron star crusts in Section 10.2 using the fully-4D covariant formulation of Carter \& Chamel [74, 75, 76]. Entrainment effects and superfluidity will be discussed in Sections 10.3 and 10.4, respectively.

\subsection{Variational formulation of multi-fluid hydrodynamics}

In the convective variational approach of hydrodynamics developed by Carter [70, 80], and recently reviewed by Gourgoulhon [174] and Andersson \& Comer [15], the dynamic equations are obtained from an action principle by considering variations of the fluid particle trajectories. First developed in the context of general relativity, this formalism has been adapted to the Newtonian framework in the usual 3+1 spacetime decomposition by Prix [342, 343]. As shown by Carter \& Chamel in a series of papers $[74,75,76]$, the Newtonian hydrodynamic equations can be written in a very concise and elegant form in a fully-4D covariant framework. Apart from facilitating the comparison between relativistic and nonrelativistic fluids, this approach sheds a new light on Newtonian hydrodynamics following the steps of Elie Cartan, who demonstrated in the 1920's that the effects of gravitation in Newtonian theory can be expressed in geometric terms as in general relativity.

The variational formalism of Carter provides a very general framework for deriving the dynamic equations of any fluid mixture and for obtaining conservation laws, using exterior calculus. In particular, this formalism is very well suited to describing superfluid systems, like laboratory superfluids or neutron star interiors, by making a clear distinction between particle velocities and the corresponding momenta (see the discussion in Section 8.3.6).

The dynamics of the system (either in relativity or in the Newtonian limit) is thus governed by a Lagrangian density $\Lambda$, which depends on the particle 4-currents $n_{\mathrm{x}}^{\mu}=n_{\mathrm{x}} u_{\mathrm{x}}^{\mu}$, where $n_{\mathrm{x}}$ and $u_{\mathrm{x}}^{\mu}$ are the particle number density and the 4-velocity of the constituent $\mathrm{X}$, respectively. We will use Greek letters for spacetime indices with the Einstein summation convention for repeated indices. The index X runs over the different constituents in the system. Note that repeated chemical indices $\mathrm{X}$ will not mean summation unless explicitly specified.

The dynamic equations for a mixture of several interacting fluids (coupled by entrainment effects) can be obtained by requiring that the action integral

$$
\mathcal{S}=\int \Lambda \mathrm{d} \mathcal{V}
$$

(where $\mathcal{V}$ is the 4 -volume element) be stationary under variations of the 4-currents $n_{\mathrm{x}}^{\mu}$. These variations are not arbitrary because they have to conserve the number of particles. In classical mechanics of point-like particles, the equations of motion can be deduced from an action integral 
by considering displacements of the particle trajectories. Likewise, considering variations of the 4-currents induced by displacements of the fluid-particle worldlines yield

$$
n_{\mathrm{x}}^{\nu} \varpi_{\nu \mu}^{\mathrm{x}}+\pi_{\mu}^{\mathrm{x}} \nabla_{\nu} n_{\mathrm{x}}^{\nu}=f_{\mu}^{\mathrm{x}}
$$

where $\nabla_{\mu}$ denotes the covariant derivative. $\pi_{\mu}^{\mathrm{x}}$, defined by

$$
\pi_{\mu}^{\mathrm{x}}=\frac{\partial \Lambda}{\partial n_{\mathrm{x}}^{\mu}}
$$

is the 4-momentum per particle associated with the 4-current $n_{\mathrm{x}}^{\mu}, \varpi_{\nu \mu}^{\mathrm{x}}$ is the vorticity 2-form defined by the exterior derivative of the corresponding 4-momentum

$$
\varpi_{\mu \nu}^{\mathrm{x}}=2 \nabla_{[\mu} \pi_{\nu]}^{\mathrm{x}}=\nabla_{\mu} \pi_{\nu}^{\mathrm{x}}-\nabla_{\nu} \pi_{\mu}^{\mathrm{x}}
$$

and $f_{\mu}^{\mathrm{x}}$ is the (nongravitational) 4-force density acting on the constituent $\mathrm{X}$. Equation (222) is the generalization to fluids of the definition introduced in classical Lagrangian mechanics for the momentum of point-like particles. This equation shows that momentum and velocity are intrinsically different mathematical objects since the former is a co-vector while the latter is a vector. The vorticity 2 -form is closely analogous to the electromagnetic 2 -form $F_{\mu \nu}$. Equation (221) is the covariant generalization of Euler's equation to multi-fluid systems. The stress-energy-momentum tensor of this multi-fluid system is given by

$$
T_{\nu}^{\mu}=\Psi \delta_{\nu}^{\mu}+\sum_{\mathrm{x}} n_{\mathrm{x}}^{\mu} \pi_{\nu}^{\mathrm{x}}
$$

where the generalized pressure $\Psi$ is defined by

$$
\Psi=\Lambda-\sum_{\mathrm{x}} n_{\mathrm{x}}^{\mu} \pi_{\mu}^{\mathrm{x}}
$$

Note that so far we have made no assumptions regarding the spacetime geometry so that Equations (221), (224) and (225) are valid for both relativistic and nonrelativistic fluids. The presence of a frozen-in magnetic field and the elasticity of the solid crust can be taken into account within the same variational framework both in (special and general) relativity [73, 85] and in the Newtonian limit $[73,72]$.

\subsection{Two-fluid model of neutron star crust}

In this section, we will review the simple model for neutron star crust developed by Carter, Chamel \& Haensel [79] (see also Chamel \& Carter [94]). The crust is treated as a two-fluid mixture containing a superfluid of free neutrons (index f) and a fluid of nucleons confined inside nuclear clusters (index c), in a uniform background of degenerate relativistic electrons. This model includes the effects of stratification (variation of the crust structure and composition with depth; see Section 3) and allows for entrainment effects (Section 8.3.7) that have been shown to be very strong [90]. However, this model does not take into account either the elasticity of the crust or magnetic fields. For simplicity, we will restrict ourselves to the case of zero temperature and we will not consider dissipative processes (see, for instance, Carter \& Chamel [76], who have discussed this issue in detail and have proposed a three-fluid model for hot neutron star crust).

This model has been developed in the Newtonian framework, since relativistic effects are expected to be small in crust layers, but using a $4 \mathrm{D}$ fully-covariant formulation in order to facilitate 
the link with relativistic models of neutron star cores [93]. In Newtonian theory, the 4-velocities are defined by

$$
u_{\mathrm{x}}^{\mu}=\frac{\mathrm{d} x^{\mu}}{\mathrm{d} t},
$$

$t$ being the "universal" time. The components of the 4 -velocity vectors have the form $u_{\mathrm{x}}^{0}=1$, $u_{\mathrm{x}}^{i}=v_{\mathrm{x}}^{i}$ in "Aristotelian" coordinates (representing the usual kind of $3+1$ spacetime decomposition with respect to the rest frame of the star). This means that the time components of the 4currents are simply equal to the corresponding particle number densities $n_{\mathrm{x}}^{0}=n_{\mathrm{x}}$, while the space components are those of the current 3 -vector $n_{\mathrm{x}}^{i}=n_{\mathrm{x}} v_{\mathrm{x}}^{i}$ (using Latin letters $i, j, \ldots$ for the space coordinate indices).

The basic variables of the two-fluid model considered here are the particle 4-current vectors $n_{\mathrm{c}}^{\mu}$, $n_{\mathrm{f}}^{\mu}$ and the number density $n_{\mathrm{N}}$ of nuclear clusters, which accounts for stratification effects. For clusters with mass number $A$, we have the relation $n_{\mathrm{c}}=A n_{\mathrm{N}}$. In the following we will neglect the small neutron-proton mass difference and we will write simply $m$ for the nucleon mass (which can be taken as the atomic mass unit, for example). The total mass density is thus given by $\rho=m\left(n_{\mathrm{c}}+n_{\mathrm{f}}\right)$. The Lagrangian density $\Lambda$, which contains the microphysics of the system, has been derived by Carter, Chamel \& Haensel [79, 78].

The general dynamic equations (221) are given, in this case, by

$$
\begin{aligned}
& n_{\mathrm{f}}^{\nu} \varpi_{\nu \mu}^{\mathrm{f}}+\pi_{\mu}^{\mathrm{f}} \nabla_{\nu} n_{\mathrm{f}}^{\nu}=f_{\mu}^{\mathrm{f}}, \\
& n_{\mathrm{c}}^{\nu} \varpi_{\nu \mu}^{\mathrm{c}}+\pi_{\mu}^{\mathrm{c}} \nabla_{\nu} n_{\mathrm{c}}^{\nu}=f_{\mu}^{\mathrm{c}} .
\end{aligned}
$$

The time components of the 4-momentum co-vectors $\pi_{\mu}^{\mathrm{x}}$ are interpretable as the opposite of the chemical potentials of the corresponding species in the Áristotelian frame, while the space components coincide with those of the usual 3-momentum co-vectors $p_{i}^{\mathrm{x}}$, defined by

$$
p_{i}^{\mathrm{x}}=\frac{\partial \Lambda}{\partial n_{\mathrm{x}}^{i}} .
$$

In general, as a result of entrainment effects [20], the momentum co-vector $p_{i}^{\mathrm{X}}$ can be decomposed into a purely kinetic part and a chemical part,

$$
p_{i}^{\mathrm{X}}=m_{\mathrm{x}} v_{\mathrm{x} i}+\chi_{i}^{\mathrm{x}} .
$$

The chemical momentum $\chi_{i}^{\mathrm{x}}$ arises from interactions between the particles constituting the fluids, and is defined by

$$
\chi_{i}^{\mathrm{x}}=\frac{\partial \Lambda_{\mathrm{int}}}{\partial n_{\mathrm{x}}^{i}} .
$$

In this case, $\Lambda_{\text {int }}$ is the internal contribution to the Lagrangian density defined by

$$
\Lambda_{\text {int }}=\Lambda-\Lambda_{\text {kin }},
$$

where

$$
\Lambda_{\mathrm{kin}}=\frac{1}{2} \sum_{\mathrm{x}} m_{\mathrm{x}} n_{\mathrm{x}} v_{\mathrm{x}}^{2} .
$$

According to the Galilean invariance, $\Lambda_{\text {int }}$ can only depend on the relative velocities between the fluids, which implies the following Noether identity

$$
\sum_{\mathrm{x}} n_{\mathrm{x}} \chi_{j}^{\mathrm{x}} \gamma^{i j}=0
$$


where $\gamma^{i j}$ is the Euclidean space metric. Consequently, unlike the individual momenta (230), the total momentum density is simply given by the sum of the kinetic momenta

$$
\sum_{\mathrm{x}} n_{\mathrm{x}} p_{j}^{\mathrm{x}} \gamma^{i j}=\sum_{\mathrm{x}} n_{\mathrm{x}} m_{\mathrm{x}} v_{\mathrm{x}}^{i}
$$

Let us stress that entrainment is a nondissipative effect and is different from drag.

The cluster 4-momentum co-vector is purely timelike since the Lagrangian density depends only on $n_{\mathrm{N}}$. It can thus be written as $\pi_{\mu}^{\mathrm{N}}=-\mu_{\mathrm{N}} t_{\mu}$, where $t_{\mu}=\nabla_{\mu} t$ is the gradient of the universal time $t$ and $\mu_{\mathrm{N}}$ is a cluster potential, whose gradient leads to stratification effects. The dynamic equation of the nuclear clusters therefore reduces to

$$
n_{\mathrm{N}} \nabla_{\mu} \mu_{\mathrm{N}}-t_{\mu} \nabla_{\nu}\left(n_{\mathrm{N}}^{\nu} \mu_{\mathrm{N}}\right)=f_{\mu}^{\mathrm{N}}
$$

where $n_{\mathrm{N}}^{\mu}=n_{\mathrm{N}} u_{\mathrm{c}}^{\mu}$. The space components of the 4 -force density co-vectors $f_{\mu}^{\mathrm{f}}, f_{\mu}^{\mathrm{c}}$ and $f_{\mu}^{\mathrm{N}}$ coincide with those of the usual 3-force density co-vectors while the time components are related to the rate of energy loss as discussed in more detail by Carter \& Chamel [76]. In the nondissipative model considered here, the total external force density co-vector vanishes:

$$
f_{\nu}^{\mathrm{f}}+f_{\nu}^{\mathrm{c}}+f_{\nu}^{\mathrm{N}}=0
$$

At this point, let us remark that, in general, the total force density co-vector may not vanish due to elastic crustal stresses, as shown by Chamel \& Carter [94]. Moreover for a secular evolution of pulsars, it would also be necessary to account for the external electromagnetic torque.

Both the cluster number and baryon number have to be conserved:

$$
\begin{gathered}
\nabla_{\mu} n_{\mathrm{N}}^{\mu}=0, \\
\nabla_{\mu} n_{\mathrm{f}}^{\mu}+\nabla_{\mu} n_{\mathrm{c}}^{\mu}=0 .
\end{gathered}
$$

On a short time scale, relevant for pulsar glitches or high frequency oscillations, it can be assumed that the composition of the crust remains frozen, i.e., the constituents are separately conserved so that we have the additional condition

$$
\nabla_{\mu} n_{\mathrm{f}}^{\mu}=0 .
$$

However, on a longer time scale, the free and confined nucleon currents may not be separately conserved owing to electroweak interaction processes, which transform neutrons into protons and vice versa. The relaxation times are strongly dependent on temperature [428] and on superfluidity [414]. A more realistic assumption in such cases is therefore to suppose that the system is in equilibrium, which can be expressed by

$$
\mathcal{A}=0
$$

where the chemical affinity $\mathcal{A}[76]$ is defined by the chemical potential difference in the crust rest frame

$$
\mathcal{A}=u_{\mathrm{c}}^{\nu}\left(\pi_{\nu}^{\mathrm{f}}-\pi_{\nu}^{\mathrm{c}}\right)
$$

\subsection{Entrainment and effective masses}

The momenta of the free superfluid neutrons and of the confined nucleons can be expressed in terms of the velocities as

$$
\begin{aligned}
& \boldsymbol{p}^{\mathbf{f}}=m_{\mathrm{f}}^{\star} \boldsymbol{v}_{\mathbf{f}}+\left(m-m_{\mathrm{f}}^{\star}\right) \boldsymbol{v}_{\mathbf{c}}, \\
& \boldsymbol{p}^{\mathbf{c}}=m_{\mathrm{c}}^{\star} \boldsymbol{v}_{\mathbf{c}}+\left(m-m_{\mathrm{c}}^{\star}\right) \boldsymbol{v}_{\mathbf{f}},
\end{aligned}
$$


where $m_{\mathrm{f}}^{\star}$ and $m_{\mathrm{c}}^{\star}$ are dynamic effective masses (for a generalization to relativistic fluids, see Chamel [93]) so that in the crust (resp. neutron superfluid) rest frame we have $\boldsymbol{p}^{\mathbf{f}}=m_{\mathrm{f}}^{\star} \boldsymbol{v}_{\mathbf{f}}$ (resp. $\left.\boldsymbol{p}^{\mathbf{c}}=m_{\mathrm{c}}^{\star} \boldsymbol{v}_{\mathbf{c}}\right)$. These effective masses arise because of the momentum transfer between the free neutrons and the nuclear lattice (Bragg scattering). Due to Galilean invariance, these effective masses are not independent but are related by

$$
m_{\mathrm{f}}^{\star}-m=\frac{\rho_{\mathrm{c}}}{\rho_{\mathrm{f}}}\left(m_{\mathrm{c}}^{\star}-m\right),
$$

where $\rho_{\mathrm{f}}$ and $\rho_{\mathrm{c}}$ are the mass densities of the neutron superfluid and confined nucleons, respectively. This entails that the total momentum density is simply $\rho_{\mathrm{f}} \boldsymbol{v}_{\mathbf{f}}+\rho_{\mathrm{c}} \boldsymbol{v}_{\mathbf{c}}$. These dynamic effective masses can be expressed directly from the internal Lagrangian density $\Lambda_{\text {int }}$ of the system by (X=f,c)

$$
\frac{m_{\star}^{\mathrm{x}}}{m}=1+\frac{2}{\rho_{\mathrm{x}}} \frac{\partial \Lambda_{\mathrm{int}}}{\partial \omega^{2}},
$$

where $\rho_{\mathrm{x}}=n_{\mathrm{x}} m$ and $\boldsymbol{\omega}=\boldsymbol{v}_{\mathbf{f}}-\boldsymbol{v}_{\mathbf{c}}$ is the relative velocity between the neutron superfluid and the crust.

Alternatively, we could introduce different kinds of effective masses $m_{\mathrm{f}}^{\sharp}$ and $m_{\mathrm{c}}^{\sharp}$, so that the momentum and the velocity of one fluid are aligned in the momentum rest frame of the other fluid,

$$
\begin{aligned}
& m_{\mathrm{f}}^{\sharp}-m=\frac{m}{m_{\mathrm{c}}^{\star}}\left(m_{\mathrm{f}}^{\star}-m\right), \\
& m_{\mathrm{c}}^{\sharp}-m=\frac{m}{m_{\mathrm{f}}^{\star}}\left(m_{\mathrm{c}}^{\star}-m\right) .
\end{aligned}
$$

This shows, in particular, that these effective masses are either all greater or all smaller than the nucleon mass $m$. From a stability analysis, Carter, Chamel and Haensel [79] proved that these effective masses obey the following inequalities $(\mathrm{X}=\mathrm{f}, \mathrm{c})$

$$
\begin{aligned}
& \frac{m_{\mathrm{x}}^{\star}}{m}>\frac{\rho_{\mathrm{x}}}{\rho}, \\
& \frac{m_{\mathrm{x}}^{\sharp}}{m}<\frac{\rho}{\rho_{\mathrm{x}}},
\end{aligned}
$$

where $\rho=\rho_{\mathrm{f}}+\rho_{\mathrm{c}}$ is the total mass density. Microscopic calculations carried out by Chamel [90, 91], using for the first time the band theory of solids (see Section 3.2.4), show that these effective masses can be much larger than the bare nucleon mass $m$. This is in sharp contrast to the situation encountered in the liquid core, where effective masses are slightly smaller than $m$ (see, for instance, Chamel \& Haensel [95] and references therein). Note, however, that the relativistic effective neutron mass can be slightly larger than the bare mass in the liquid core, as recently shown by Chamel [93].

\subsection{Neutron superfluidity}

As already discussed in Section 8.3.2, a superfluid is locally characterized by condition (155), which, in the $4 \mathrm{D}$ covariant framework $[74,75]$, reads

$$
\varpi_{\mu \nu}^{\mathrm{f}}=0
$$

However, at length scales that are large compared to the intervortex spacing, the neutron vorticity 2 -form will not vanish. Since the vorticity of superfluid is carried by quantized vortex lines (as 
illustrated in Figure 60), mathematically this means that the Lie derivative of the vorticity 2-form along the 4 -velocity vector $u_{v}^{\mu}$ of the vortex lines, vanishes:

$$
\vec{u}_{v} £ \varpi_{\mu \nu}^{\mathrm{f}}=0 .
$$

This condition is equivalent to

$$
u_{v}^{\mu} \varpi_{\mu \nu}^{\mathrm{f}}=0
$$

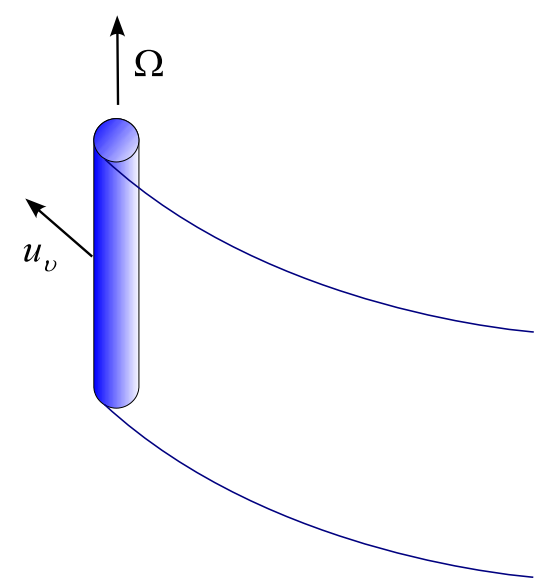

Figure 60: Sketch of the 2-surface swept out by a quantized vortex line moving with the 4-velocity $u_{v}$; $\Omega$ is the superfluid angular velocity.

Let us remark that the definition of neutrons that have to be counted as "free" is not unique and there is some arbitrariness in the above model. Nonetheless, it can be shown that the 4-momentum co-vector $\pi_{\mu}^{\mathrm{f}}$ of the neutron superfluid is invariant under such "chemical" readjustments and the above superfluidity conditions are well defined [79]. Note also that these conditions are valid for both relativistic and nonrelativistic superfluids.

As discussed by Chamel \& Carter [94], there are two cases, which are consistent with the nondissipative models considered here. The first possibility is that the neutron vortices are free and are co-moving with the superfluid, i.e. $u_{v}^{\mu}=u_{\mathrm{f}}^{\mu}$. On a sufficiently short time scale, it may be further assumed that the free neutron current is conserved, which implies from Equations (221) and (253) that the force $f_{\mu}^{\mathrm{f}}$ vanishes. Since no external force is supposed to be exerted on the system, the force acting on the confined nucleons also vanishes. However, on longer time scales, as discussed in Section 10.2, it would be more appropriate to replace Equation (240) by the equilibrium condition Equation (241). In this case, there will still be a force acting on the superfluid (hence, also a force, acting on the confined nucleons) owing to the conversion of free neutrons into confined protons and vice versa. The other possibility is that the vortices are pinned to the crust, so that $u_{v}^{\mu}=u_{\mathrm{c}}^{\mu}$. As shown by Chamel \& Carter [94], the pinning condition $u_{\mathrm{c}}^{\mu} \varpi_{\mu \nu}^{\mathrm{f}}=0$ is equivalent to imposing that the individual vortices be subject to the corresponding Magnus force.

The dynamics of the neutron superfluid vortices in the crust play a major role in the understanding of pulsar glitches and post-glitch relaxation. Due to entrainment effects, the distribution of vortices is not simply given by Equation (163), but also depends on the angular velocity of the crust according to the following formula derived by Chamel \& Carter [94]:

$$
n_{v}=\frac{2 m \Omega_{\mathrm{f}}}{\pi \hbar}+\frac{2 m}{\pi \hbar}\left(\frac{m_{\mathrm{f}}^{\star}}{m}-1\right)\left(\Omega_{\mathrm{f}}-\Omega_{\mathrm{c}}\right)
$$


assuming that the neutron superfluid and the crust are uniformly rotating around the same axis with angular velocities $\Omega_{\mathrm{f}}$ and $\Omega_{\mathrm{c}}$, respectively. In addition, spatial variations of the effective masses are neglected. Since the superfluid rotates faster than the crust and since the dynamic effective neutron mass is larger than the bare mass, the entrainment effects increase the surface density of the vortices (whereas the entrainment effects decrease the surface density of neutron vortices in the liquid core). 


\section{Neutrino Emission}

\subsection{Neutrino emission processes - an overview}

Table 6: Main neutrino emission processes in neutron star crusts. Symbols: $\gamma$ stands for a photon or a plasmon; $(A, Z)$ stands for a nucleus with charge number $Z$ and mass number $A$; lepton symbol $x=e, \mu, \tau$; neutron quasiparticle (neutron-like elementary excitation) in superfluid neutron gas is denoted by $\widetilde{n}$.

\begin{tabular}{c|c}
\hline Process name & Reaction \\
\hline$e^{-} e^{+}$pair annihilation & $e^{-}+e^{+} \longrightarrow \nu_{x}+\bar{\nu}_{x}$ \\
Plasmon decay & $\gamma \longrightarrow \nu_{x}+\bar{\nu}_{x}$ \\
Photoneutrino emission & $\gamma+e^{-} \longrightarrow e^{-}+\nu_{x}+\bar{\nu}_{x}$ \\
Electron synchrotron radiation & $e^{-} \longrightarrow e^{-}+\nu_{x}+\bar{\nu}_{x}$ \\
Electron-nucleus Bremsstrahlung & $e^{-}+(A, Z) \longrightarrow e^{-}+(A, Z)+\nu_{x}+\bar{\nu}_{x}$ \\
Cooper pair formation & $\widetilde{n}+\widetilde{n} \longrightarrow \nu_{x}+\bar{\nu}_{x}$ \\
\hline
\end{tabular}

There is a great wealth of neutrino emission processes in hot neutron star crusts. These processes are associated with weak interaction involving electrons, positrons, nuclei, and free nucleons. As soon as the crust becomes neutrino-transparent, which occurs in about a minute after neutron star birth, these neutrinos freely leave the crust, taking away thermal energy, and contributing in this way to crust cooling. Let us notice that, because of a finite thermal equilibration timescale, during the first few decades of a neutron star's life the thermal evolution of the crust is decoupled from that of the liquid core.

In this section we do not pretend to give a complete review of neutrino emission processes. We will limit ourselves to a brief presentation of six main neutrino emission mechanisms, listed in Table 6. Some other neutrino emission processes are briefly mentioned in Section 11.8. We will dwell on the qualitative features of main contributions to the neutrino emissivity of the crust, $Q_{\nu}$, which is defined as the energy radiated in neutrinos (in erg) from $1 \mathrm{~cm}^{3}$ during $1 \mathrm{~s}$. In particular, we will discuss, at the qualitative level, the density and temperature dependences of the contributions from a given process. A reader interested in a more complete and detailed description of neutrino emission processes from the crust is referred to the review of Yakovlev et al. [428].

Processes reviewed in Sections 11.2-11.6 have been studied mainly at $\boldsymbol{B}=0$. The effects of $\boldsymbol{B}$ on $Q_{\nu}$ were calculated only for some processes, and will be briefly mentioned at the end of the corresponding sections. The presence of $\boldsymbol{B}$ makes possible the synchrotron radiation of electrons, considered in Section 11.7.

A summary of the $\rho-T$ dependence of different contributions to $Q_{\nu}$, and a discussion of their relative importance in different regions of the $\rho-T$ plane, are presented in Section 11.9. 


\subsection{Electron-positron pair annihilation}

This process was proposed by Chiu \& Morrison [98]. It requires the presence of positrons and is, therefore, important at high temperatures and low densities. Such conditions prevail in the outer layer of a newly-born neutron star. The matter there is opaque to photons. Electrons, positrons and photons are in thermodynamic equilibrium, with number densities $n_{e^{-}}, n_{e^{+}}, n_{\gamma}$, and the corresponding chemical potentials $\mu_{e^{-}}, \mu_{e^{+}}, \mu_{\gamma}$, respectively. As the number of photons is not fixed, their chemical potential $\mu_{\gamma}=0$. Therefore, equilibrium with respect to reactions

$$
e^{+}+e^{-} \leftrightarrow 2 \gamma
$$

implies $\mu_{e^{-}}=-\mu_{e^{+}}$. Electrons and positrons can be treated as ideal relativistic Fermi gases. An electron or a positron of momentum $\boldsymbol{p}$ has energy

$$
\epsilon(\boldsymbol{p})=\epsilon(p)=\sqrt{\left(m_{e} c^{2}\right)^{2}+p^{2} c^{2}} .
$$

The electron and positron number densities are given by (writing $\mu_{e^{-}}=\mu_{e}$ )

$$
n_{e^{-}}=\frac{1}{\pi^{2} \hbar^{3}} \int_{0}^{\infty} \mathrm{d} p \frac{p^{2}}{1+\exp \left[\left(\epsilon(p)-\mu_{e}\right) / k_{\mathrm{B}} T\right]},
$$

and

$$
n_{e^{+}}=\frac{1}{\pi^{2} \hbar^{3}} \int_{0}^{\infty} \mathrm{d} p \frac{p^{2}}{1+\exp \left[\left(\epsilon(p)+\mu_{e}\right) / k_{\mathrm{B}} T\right]} .
$$

Charge neutrality implies

$$
n_{e^{+}}+n_{e^{-}}=n_{\mathrm{N}} Z,
$$

where $n_{\mathrm{N}}$ is the density of nuclei. The calculation of the neutrino emissivity $Q_{\text {pair }}$ from reactions

$$
e^{+}+e^{-} \longrightarrow \nu_{x}+\bar{\nu}_{\ell}, \quad \ell=e, \mu, \tau,
$$

is described in detail, e.g., in Yakovlev et al. [428]. Here we limit ourselves to a qualitative discussion of two limiting cases. Let us first consider the case of nondegenerate electrons and positrons, $k_{\mathrm{B}} T>\mu_{e}$; such conditions prevail in the supernova shock and in the shocked envelope of a newly born proto-neutron star. Then, $n_{e^{+}} \approx n_{e^{-}} \gg n_{\mathrm{N}} Z$. The mean energies of electrons, positrons, photons and neutrinos are then "thermal", $\langle\epsilon\rangle \sim k_{\mathrm{B}} T$. The cross section $\sigma_{e^{+} e^{-} \rightarrow \nu \bar{\nu}}$ for process (260) is quadratic in the center-of-mass energy. Therefore, the temperature dependence of $Q_{\nu}^{\text {pair }}$ can be evaluated as

$$
Q_{\nu}^{\text {pair }} \propto\left\langle\epsilon_{\nu}\right\rangle n_{e^{-}} n_{e^{+}} \sigma_{e^{+} e^{-} \rightarrow \nu \bar{\nu}} \propto T \times T^{3} \times T^{3} \times T^{2} \propto T^{9} .
$$

Let us now consider the opposite limit of degenerate ultra-relativistic electrons, $k_{\mathrm{B}} T \ll \mu_{e}$ and $\mu_{e} \gg m_{e} c^{2}$. The positron density is then exponentially small. This is because $\mu_{e^{+}}=-\mu_{e}$ is large and negative, so that $n_{e^{+}} \propto \exp \left(-\mu_{e} / k_{\mathrm{B}} T\right) \ll n_{e^{-}}$. Therefore, the pair annihilation process is strongly suppressed for degenerate electrons, with decreasing temperature or increasing density. Detailed formulae for $Q_{\nu}^{\text {pair }}$, valid in different density-temperature domains, are given in [428].

The pair annihilation process can be affected by a strong magnetic field $\boldsymbol{B}$. General expressions for $Q_{\nu}^{\text {pair }}$ for arbitrary $\boldsymbol{B}$ were derived by Kaminker et al. [230, 228]. In these papers one can also find practical expressions for a hot, nondegenerate plasma in arbitrary $\boldsymbol{B}$, as well as interpolating expressions for $Q_{\nu}^{\text {pair }}$ in a plasma of any degeneracy and in any $\boldsymbol{B}$. In a hot, nondegenerate plasma with $T \gtrsim 10^{10} \mathrm{~K}, B \gg 10^{15} \mathrm{G}$ must be huge to affect $Q_{\nu}^{\text {pair }}$. However, at $T \lesssim 10^{9} \mathrm{~K}$, even $B \sim 10^{14} \mathrm{G}$ may quantize the motion of positrons and increase substantially their number density. Consequently, $B \sim 10^{14} \mathrm{G}$ strongly increases $Q_{\nu}^{\text {pair }}$ al low densities. This is visualized in Figure 62. 


\subsection{Plasmon decay}

Quanta of electromagnetic waves in a plasma have different properties than in vacuum. They are called (electron) plasmons, and appear in two basic modes. We first consider the case $\boldsymbol{B}=0$. Those modes, which consist of transverse oscillations, are called transverse plasmons. The relation between the frequency of transverse plasmons $\omega$ and their wave-number $k=2 \pi / \lambda$ (where $\lambda$ is the wavelength) for the simplest case of nonrelativistic electrons is

$$
\omega=\sqrt{\omega_{\mathrm{p} e}^{2}+k^{2} c^{2}},
$$

where $\omega_{\mathrm{p} e}$ is the electron plasma frequency (12). Only plasmons with $\omega>\omega_{\mathrm{p} e}$ can propagate in the crust. At a given temperature $T$, the number density of plasmons is given by the Bose-Einstein formula

$$
n_{\gamma} \propto \int \mathrm{d} p \frac{p^{2}}{\mathrm{e}^{\hbar \omega / k_{\mathrm{B}} T}-1} .
$$

While a photon in vacuum cannot decay into a neutrino-antineutrino pair, a plasmon in a plasma can,

$$
\gamma \rightarrow \nu_{x}+\bar{\nu}_{\ell}, \quad \ell=e, \mu, \tau
$$

This process of neutrino emission was first considered in detail by Inman \& Ruderman [209]. For $T \ll T_{\text {pe }}$, the value of $Q_{\nu}^{\text {plas }}$ is strongly temperature dependent,

$$
Q_{\nu}^{\text {plas }} \propto \exp \left(-T_{\mathrm{p} e} / T\right)
$$

where $T_{\mathrm{pe}}$ is the electron plasma temperature defined by Equations (12) and (13). For $T \ll T_{\text {pe }}$, the plasmon decay process is therefore negligible. It is also strongly density dependent through $T_{\mathrm{pe}}$ in Equation (265). Generally, $Q_{\nu}^{\text {plas }}$ is switched-off by decreasing temperature and increasing density. Detailed formulae for $Q_{\nu}^{\text {plas }}$ can be found in [428].

The plasmon decay is influenced by a strong magnetic field, because $\boldsymbol{B}$ modifies the plasma dispersion relation (relation between plasmon frequency $\omega$ and its wavenumber $k$ ). In particular, new plasma modes may appear. The effects of magnetic fields are important if $\omega_{B} \equiv e B /\left(m_{e}^{*} c\right)>$ $\omega_{\text {pe }}$. At $\rho \sim 10^{11} \mathrm{~g} \mathrm{~cm}^{-3}$ this requires $B \gtrsim 3 \times 10^{15} \mathrm{G}$. The magnitude of $B$ required to modify the plasmon dispersion relation grows as $\rho^{2 / 3}$.

\subsection{Photoneutrino emission}

This process differs from the plasmon decay described in Section 11.3, by the participation of an electron,

$$
\gamma+e^{-} \longrightarrow \nu_{\ell}+\bar{\nu}_{\ell}+e^{-}, \quad \ell=e, \mu, \tau
$$

The process was proposed by Ritus [347, 348] and Chiu \& Stabler [99]; the most recent formulae for $Q_{\nu}^{\text {phot }}$ were derived by Itoh et al. [215, 216, 217]. Formula (263) implies that if $T \ll T_{\text {pe }}$ then $n_{\gamma} \propto \mathrm{e}^{-T_{\mathrm{p} e} / T}$. Therefore, $Q_{\nu}^{\mathrm{phot}}$ is exponentially suppressed with decreasing temperature or increasing density. Additional damping of $Q_{\nu}^{\text {phot }}$ results from electron degeneracy. However, if electrons are nondegenerate, $Q_{\nu}^{\text {phot }}$ can be stronger than $Q_{\nu}^{\text {plas }}$

\subsection{Neutrino Bremsstrahlung from electron-nucleus collisions}

Proposed by Pontecorvo [335, 336] and Gandelman [154, 155], neutrino Bremsstrahlung from electron-nucleus collisions is one of the major neutrino emission mechanisms in the crust. The process can be written as

$$
e^{-}+(A, Z) \longrightarrow e^{-}+(A, Z)+\nu_{\ell}+\bar{\nu}_{\ell}, \quad \ell=e, \mu, \tau .
$$


With obvious notations, the total momentum of an initial state is $\boldsymbol{P}=\boldsymbol{p}_{e}+\boldsymbol{p}_{A}$, and that of a final state $\boldsymbol{P}^{\prime}=\boldsymbol{p}_{e}^{\prime}+\boldsymbol{p}_{A}^{\prime}+\boldsymbol{p}_{\nu_{\ell}}+\boldsymbol{p}_{\bar{\nu}_{\ell}}^{\prime}$. The corresponding total energies will be denoted by $E$ and $E^{\prime}$. We first consider the case of $T>T_{\mathrm{m}}$, when nuclei form a Coulomb liquid, $T_{\mathrm{m}}$ being the melting temperature of the crust defined by Equation (15). Let us also neglect, for the sake of simplicity, the Coulomb correlations between ions. The neutrino emissivity from process (267) is then calculated by integrating the energy emission rate over initial and final momenta,

$$
\begin{aligned}
Q_{\nu}^{\text {Brem }} & \propto \int \mathrm{d}^{3} p_{e} \mathrm{~d}^{3} p_{e}^{\prime} \mathrm{d}^{3} p_{\nu} \mathrm{d}^{3} p_{\bar{\nu}} \mathrm{d}^{3} p_{A} \mathrm{~d}^{3} p_{A}^{\prime} \\
& \times W_{\text {Brem }} \delta\left(E^{\prime}-E\right) \delta\left(\boldsymbol{P}^{\prime}-\boldsymbol{P}\right) f_{A} f_{e}\left[1-f_{e^{\prime}}\right]\left(\epsilon_{\nu}+\epsilon_{\bar{\nu}}\right),
\end{aligned}
$$

where $f_{A}$ is the Boltzmann distribution of ions (nuclei), $f_{e}$ is the Fermi-Dirac distribution for electrons,

$$
f_{e}(\boldsymbol{p})=\frac{1}{\mathrm{e}^{\left[\left(\epsilon(p)-\mu_{e}\right) / k_{\mathrm{B}} T\right]}+1},
$$

and $W_{\text {Brem }}$ is the square of the transition amplitude corresponding to elementary process (267). The factor $\left[1-f_{e^{\prime}}\right]$ takes care of the Pauli exclusion principle for the electron in the final state. Let us consider the integration over the neutrino momenta. We can rewrite $\mathrm{d}^{3} p_{\nu}=\mathrm{d}^{2} \hat{\boldsymbol{p}}_{\nu} p_{\nu}^{2} \mathrm{~d} p_{\nu}$ $\left(\hat{\boldsymbol{p}}_{\nu} \equiv \boldsymbol{p}_{\nu} / p_{\nu}\right)$ and replace $p_{\nu}$ with $\epsilon_{\nu} / c$. Relevant neutrino energies are $\epsilon_{\nu} \sim k_{\mathrm{B}} T$. Electrons are degenerate, and the momenta contributing to the integral are close to the electron Fermi surface. Consequently, the integration over energies is restricted to a thin shell around $\mu_{e}$ of a thickness $\sim k_{\mathrm{B}} T$. Integration over neutrinos and antineutrinos yields a factor of $T^{3}$ each, neutrino energies give $T$, and the energy delta function removes one $T$ factor. Moreover, $W_{\text {Brem }} \propto Z^{2}$ and $n_{\mathrm{N}} \propto \rho / A$. To account for the nonideality of the plasma of nuclei, one introduces an additional dimensionless factor $L$ [181]. All in all,

$$
Q_{\nu}^{\text {Brem }} \propto T^{6} Z^{2} \rho L / A
$$

For $\Gamma \ll 1$ we have $L \approx 1$, but for a strongly coupled plasma $(\Gamma \gg 1) L$ can be significantly smaller than one (see [181]).

Things become more complicated at low temperatures. Then the electron states are no longer described by plane waves. Instead, the Bloch functions consistent with crystal symmetry should be used (see the review of band theory in Section 3.2.4). The electron energy spectrum is no longer continuous, but is formed of bands. At high $T$, the thermal motion of electrons "smears out" this band structure. However, the gaps between energy bands strongly suppress $Q_{\nu}^{\text {Brem }}$ [231]. Detailed formulae valid for different domains of the density-temperature plane can be found in [428].

Strong magnetic fields affect the motion of electrons scattered off nuclei. However, the effect of $\boldsymbol{B}$ on $Q_{\nu}^{\text {Brem }}$ has not been calculated.

\subsection{Cooper pairing of neutrons}

For temperatures $T<T_{\mathrm{c} n}$, the free neutrons in the inner crust are superfluid (see Section 8). The elementary excitations in the superfluid neutron gas, denoted by $\widetilde{n}$, are fermions. They have a specific energy spectrum, as discussed in Section 8.3.1. The elementary excitations are called (Bogoliubov) quasiparticles. The simplest excitation in a superfluid is a quasiparticle pair. Annihilation of a quasiparticle pair can go via an emission of a neutrino-antineutrino pair,

$$
\tilde{n}+\widetilde{n} \longrightarrow \nu_{\ell}+\bar{\nu}_{\ell}, \ell=e, \mu, \tau \text {. }
$$

Such a process was first considered by Flowers et al. [148]. Neglecting for simplicity the presence of the nuclear clusters and considering a uniform superfluid (see Section 8.2), the temperature dependence of the neutrino emissivity $Q_{\nu}^{\mathrm{CP}}$ from these processes is given by

$$
Q_{\nu}^{\mathrm{CP}} \propto T^{7} F\left(T / T_{\mathrm{cn}}\right)
$$


where $F(x \geq 1)=0$ and $F(x \longrightarrow 0)=0$. Detailed derivations and formulae can be found in [428].

The actual value of the numerical prefactor in Equation (272) has recently become a topic of lively discussion. Recent calculations have shown that previous results may severely overestimate $Q_{\nu}^{\mathrm{CP}}$ (see, e.g., [368, 245], and references therein). However, the actual reduction of $Q_{\nu}^{\mathrm{CP}}$ is still a matter of debate.

\subsection{Synchrotron radiation from electrons}

The magnetic field not only modifies the rates of neutrino emission processes, but also opens new channels of neutrino emission. Let us consider a coordinate system such that $\boldsymbol{B}=[0,0, B]$. The electron energy levels are then given by $\epsilon_{n}\left(p_{z}\right)=c\left(m_{e}^{2} c^{2}+2 \hbar \omega_{\mathrm{c}} m_{e} n+p_{z}^{2}\right)^{1 / 2}$, where $n$ is the Landau quantum number. The transverse component of the electron momentum is not conserved, and this allows for the electron synchrotron radiation process,

$$
e^{-}(\boldsymbol{B}) \longrightarrow e^{-}(\boldsymbol{B})+\nu_{\ell}+\bar{\nu}_{x}, \quad \ell=e, \mu, \tau,
$$

where the notation $\boldsymbol{B}$ is to remind us of the necessary presence of the magnetic field. For nonquantizing $\boldsymbol{B}\left(T \gg T_{B}\right)$ and nondegenerate nonrelativistic electrons, the neutrino emission rate is temperature independent $Q_{\nu}^{\text {syn }} \propto B^{6}$. But for a hot $\left(T \gg T_{B}\right)$ ultra-relativistic $\left(k_{\mathrm{B}} T \gg m_{e} c^{2}\right)$ electron-positron gas, we get a density independent $Q_{\nu}^{\mathrm{syn}} \propto B^{2} T^{5}$ (see [428]).

\subsection{Other neutrino emission mechanisms}

There are many other mechanisms of neutrino emission. For example, there is the possibility of $\nu \bar{\nu}$ pair Bremsstrahlung emission accompanying $n n \longrightarrow n n \nu \bar{\nu}$ scattering of dripped neutrons, and scattering of neutrons on nuclear clusters, $n(A, Z) \longrightarrow n(A, Z) \nu \bar{\nu}$. Moreover, in a newlyborn neutron star beta processes involving electrons, positrons and nuclei, e.g., $e^{-}(A, Z) \longrightarrow$ $(A, Z-1) \nu_{e},(A, Z-1) \longrightarrow(A, Z) e^{-} \bar{\nu}_{e}$, etc., are a source of neutrino emission. These are the famous Urca processes, proposed in the early 1940s; their intriguing history is described, e.g., in Section 3.3.5 of Yakovlev et al. [428]. One can also contemplate a photo-emission from nuclei, $\gamma(A, Z) \longrightarrow(A, Z) \nu \bar{\nu}$. Finally, we should also mention the interesting possibility of a very efficient neutrino emission by the direct Urca process in some "pasta layers" (see Section 3.3) near the bottom of the crust $[274,259,260,179]$. As this mechanism, restricted to a bottom layer of the neutron star crust, could be a very efficient neutrino emission channel, we will describe it in more detail.

\subsubsection{Direct Urca process in the pasta phase of the crust}

It is well known that the direct Urca process is the most efficient mechanism of neutrino emission [254]. The direct Urca reactions in a dense degenerate plasma composed mainly of neutrons, with an admixture of protons and electrons, are the neutron beta decay and the inverse reaction of electron capture on a proton,

$$
n \longrightarrow p+e^{-}+\bar{\nu}_{e}, \quad p+e^{-} \longrightarrow n+\nu_{e} .
$$

These reactions are allowed by momentum conservation, if the Fermi momenta of neutrons, protons, and electrons satisfy the triangle condition $p_{\mathrm{F} n}<p_{\mathrm{F} p}+p_{\mathrm{Fe} e}$. The triangle condition implies that the proton fraction in the npe plasma should be greater than $1 / 9 \approx 11 \%$. For the time being, we ignore whether this condition is satisfied in the cores of the most massive neutron stars. If the direct Urca (dUrca) process is forbidden, then the main neutrino emission mechanism from the nonsuperfluid neutron star core is the modified Urca (mUrca) process,

$$
n+X \longrightarrow p+X+e^{-}+\bar{\nu}_{e}, p+X+e^{-} \longrightarrow n+X+\nu_{e},
$$

Living Reviews in Relativity

http://www. livingreviews.org/Irr-2008-10 
where $X=n$ or $X=p$ is an additional "spectator" nucleon needed for momentum conservation. By strong (nuclear) interaction with $n$ or $p, X$ absorbs (supplies) the excessive (missing) momentum of the nucleons participating in the processes (275), without changing its own nucleon state. The difference in emissivities from the dUrca and mUrca processes is huge. For nonsuperfluid neutron star cores, $Q_{\nu}^{\mathrm{dUrca}} / Q_{\nu}^{\mathrm{mUrca}} \sim 10^{6} T_{9}^{-2}$, where $T_{9} \equiv T /\left(10^{9} \mathrm{~K}\right)[255]$.

If the "pasta mantle" of the crust exists (see Section 3.3), it allows for a partial opening of the dUrca process in those phases, in which the npe matter component fills most of the space. This happens in the phases with tubes or bubbles filled with a neutron gas. However, because of the periodicity of the lattice of tubes or bubbles, neutrons and protons in the npe plasma move in a periodic nuclear single-particle potential. This means that the nucleon single particle wave functions are no longer the eigenfunctions of momentum (plane waves), but have to be replaced by Bloch wave functions (see Section 3.2.4). All in all, the dUrca process becomes "slightly open" in the relevant pasta layers of the crust [259, 260, 179]. The emissivities calculated by Gusakov et al. [179] can be presented as

$$
Q_{\nu}^{\mathrm{dUrca}(\mathrm{m})}=\mathcal{R}(\rho) Q_{\nu}^{\mathrm{dUrca}(0)}(\rho, T)
$$

where $Q_{\nu}^{\mathrm{dUrca}(0)}$ is the dUrca emissivity for a homogeneous npe plasma, calculated using plane waves and ignoring momentum conservation, and $\mathcal{R}$ is the reduction factor, resulting from momentum and energy constraints in the presence of a periodic lattice of tubes or bubbles filled with a neutron gas. The calculation performed for the bubble phase shows that $\mathcal{R} \sim 10^{-5}$, but even this strong reduction still allows $Q_{\nu}^{\mathrm{dUrca}(\mathrm{m})}$ to be much larger than that from any other process of neutrino emission in the neutron star crust [179]. However, it should be stressed that $Q_{\nu}^{\mathrm{dUrca}(\mathrm{m})}$ acts in a rather thin bottom layer of the crust. In the model developed by Gusakov et al. [179], it was localized in the "Swiss-cheese" layer in the density range $\left(10^{14.14}-10^{14.16}\right) \mathrm{g} \mathrm{cm}^{-3}$. Within this layer, and at temperature $T=3 \times 10^{8} \mathrm{~K}, Q_{\nu}^{\mathrm{dUrca}(\mathrm{m})}$ exceeds all other crust emissivities by a factor of at least $10^{4}$.

\subsection{Neutrinos from the crust - summary in the $T-\rho$ plane}

In this section we will summarize results for neutrino emission from a neutron star crust. We will limit ourselves to densities $\rho \gtrsim 10^{7} \mathrm{~g} \mathrm{~cm}^{-3}$, so that electrons will always be ultra-relativistic (see Section 3).

Contributions to $Q_{\nu}$ from various neutrino emission mechanisms (except for the Cooper-pair mechanism, which will be considered later in this section) versus $\rho$ are plotted in Figures 61, 62 and 63 .

We start with the crust of a very young neutron star, with a temperature $T=3 \times 10^{9} \mathrm{~K}$ (age $\lesssim 1$ year), Figure 61 . For density $\rho \lesssim 10^{8} \mathrm{~g} \mathrm{~cm}^{-3}$, the contribution $Q_{\nu}^{\text {pair }}$ is dominant. However, with increasing density, electrons become degenerate, and positrons disappear in the matter, so that $Q_{\nu}^{\text {pair }}$ is strongly suppressed at $\rho>10^{9} \mathrm{~g} \mathrm{~cm}^{-3}$. We also notice that $Q_{\nu}^{\text {phot }}$ is never important in the inner crust, because of the strong electron degeneracy. $Q_{\nu}^{\text {plas }}$ from the plasmon decay gives the dominant contribution to $Q_{\nu}$ from $\rho \approx 10^{9} \mathrm{~g} \mathrm{~cm}^{-3}$ down to the bottom of the crust. We notice also that $Q_{\nu}^{\text {syn }}$ behaves differently than the other contributions. Namely, at $\rho \gtrsim 10^{9} \mathrm{~g} \mathrm{~cm}^{-3}$ its density dependence is very weak, and $Q_{\nu}^{\text {syn }}$ scales approximately with the magnetic field $B$ as $\propto B^{2}$. Finally, one notices jumps of $Q_{\nu}^{\text {Brem }}$ and $Q_{\nu}^{\text {plas }}$, which result from jumps in $Z$ and $A$ in the ground-state matter. As we will see, this feature is even more pronounced at lower temperatures $T$.

Let us now consider the case of a colder crust at $T=10^{9} \mathrm{~K}$, Figure 62 . Except for $Q_{\nu}^{\text {syn }}$, which is just scaled down due to the decrease of temperature, there is a dramatic change in the overall landscape. For a magnetic field $B=10^{14} \mathrm{G}, Q_{\nu}^{\text {syn }}$ dominates in the lowest-density region. On the 


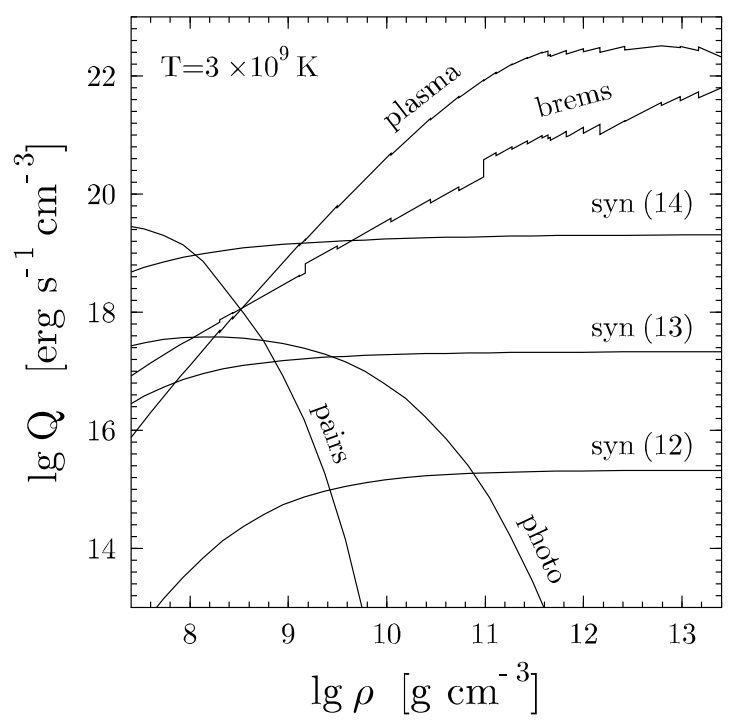

Figure 61: Neutrino emissivities associated with different mechanisms of neutrino emission acting in a neutron star crust, versus $\rho$, at temperature $T=3 \times 10^{9} \mathrm{~K}$. Numbers in parentheses indicate $\log _{10} B$. Effect of $B=10^{14} \mathrm{G}$ (and a fortiori - effect of a lower $B$ ) on $Q_{\nu}^{\text {pair }}$ is insignificant. $Q_{\nu}^{\text {plas }}, Q_{\nu}^{\text {Brem }}$, and $Q_{\nu}^{\text {phot }}$ were calculated at $B=0$. Label "syn (14)" - synchrotron radiation by electrons in constant magnetic field $B=10^{14} \mathrm{G}$, etc. Ground-state composition of the crust is assumed: Haensel \& Pichon [183] model for the outer crust, and Negele \& Vautherin [303] model for the inner crust. For further explanations see the text. From [428].

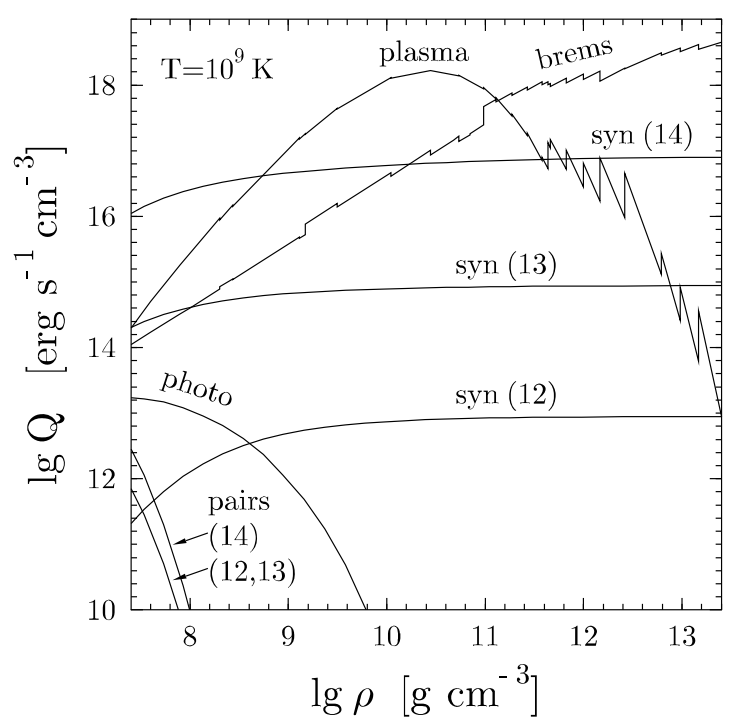

Figure 62: Same as for Figure 61, but at $T=10^{9} \mathrm{~K} . Q_{\nu}^{\text {pair }}$ is increased by $B$, as shown by the labels (14), (13), (12). Notice, that $Q_{\nu}^{\text {pair }}$ at $B=0$ is too low to be seen. From [428]. 
contrary, $Q_{\nu}^{\text {pair }}$ is of marginal importance, and is influenced by $\boldsymbol{B}$ (increases with $B$ ). Moreover, contribution of $Q_{\nu}^{\text {phot }}$ is negligible. Neglecting the effect of magnetic fields, one concludes that $Q_{\nu}^{\text {plas }}$ dominates in the outer crust, while $Q_{\nu}^{\text {Brem }}$ dominates in the inner crust. Let us notice that $Q_{\nu}^{\text {plas }}$ reaches its maximum near $10^{10.5} \mathrm{~g} \mathrm{~cm}^{-3}$ and then decreases by four orders of magnitude when the density falls below $\rho \sim 10^{13} \mathrm{~g} \mathrm{~cm}^{-3}$; this characteristic behavior is due to the $\exp \left(-T_{\mathrm{p} e} / T\right)$ factor, Equation (265). On the contrary, $Q_{\nu}^{\text {Brem }}$ rises steadily with increasing density.

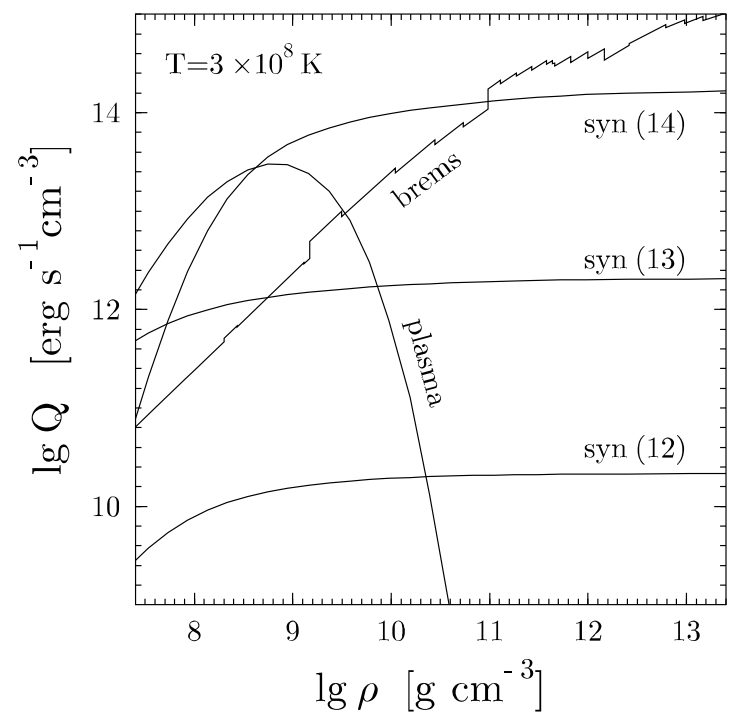

Figure 63: Same as in Figure 61, but calculated at $T=3 \times 10^{8}$ K. $Q_{\nu}^{\text {pair }}$ and $Q_{\nu}^{\text {phot }}$ are too small to be seen. From [428].

Finally, in Figure 63 we consider an even colder crust at $T=3 \times 10^{8} \mathrm{~K}$. Pair and photoneutrino constributions have disappeared completely, while $Q_{\nu}^{\text {plas }}$ dominates for $\rho<10^{9} \mathrm{~g} \mathrm{~cm}^{-3}$, whereas at higher densities, $Q_{\nu}^{\mathrm{Brem}}$ is the main source of neutrino emission. At the magnetic field $B=$ $10^{14} \mathrm{G}$, characteristic of magnetars, synchrotron radiation dominates in the density interval near $\sim 10^{10} \mathrm{~g} \mathrm{~cm}^{-3}$, but then at $\rho \gtrsim 10^{11} \mathrm{~g} \mathrm{~cm}^{-3}, Q_{\nu}^{\text {Brem }}$ becomes the strongest neutrino radiation mechanism.

Two general remarks are in order. First, as we have already mentioned, jumps in $Q_{\nu}^{\text {Brem }}$ and $Q_{\nu}^{\text {plas }}$ are due to specific factors involving $Z^{2}$ and $A$ and reflect the jumps in $Z$ and $A$ in the layered crust. For the other mechanisms, the electron chemical potential $\mu_{e}$ with its smooth dependence on $\rho$ plays the role of the crucial plasma parameter, and therefore no jumps are seen. Secondly, were the magnetic field $B \geq 10^{15} \mathrm{G}, Q_{\nu}^{\mathrm{syn}}$ would be overall dominant for $T<10^{9} \mathrm{~K}$ and $\rho>10^{9} \mathrm{~g} \mathrm{~cm}^{-3}$.

The Cooper-pair mechanism of neutrino radiation differs fundamentally from the other mechanisms of neutrino cooling, discussed above, and therefore we consider it separately. $Q_{\nu}^{\mathrm{CP}}$ depends sensitively on the interplay between temperature $T$ and the ${ }^{1} \mathrm{~S}_{0}$ pairing gap $\Delta_{\mathrm{F}}$ of the dripped neutrons. The gap itself depends on $T$, rising from zero at $T=T_{\text {cn }}$ to the asymptotic value $\Delta_{0} \equiv \Delta_{\mathrm{F}}(T=0)$ for $T \ll T_{\mathrm{cn}}$ (see Section 8.2.2). As we already discussed in Section 8.2.1, the dependence of $\Delta_{0}$ on the free neutron density, $\rho_{n}$, is very poorly understood, and this introduces a large uncertainty in the calculated values of $Q_{\nu}^{\mathrm{CP}}$. Notice that the relation $\rho_{n}(\rho)$, needed to get $Q_{\nu}^{\mathrm{CP}}(\rho)$, depends on the model of the inner crust.

Figure 64 refers to $T=10^{9} \mathrm{~K}$, a selected model of neutron superfluidity, and a selected model of the inner crust. In the BCS theory (Section 8.2.1), the maximum of $\Delta_{0}(\rho)$, denoted by $\Delta_{0}^{\max }$, corresponds to the maximum of $T_{\mathrm{c} n}(\rho)$, given by $T_{\mathrm{c} n}^{\max }=0.5669 \Delta_{0}^{\max } / k_{\mathrm{B}}$ (Section 8.2.2). In the 


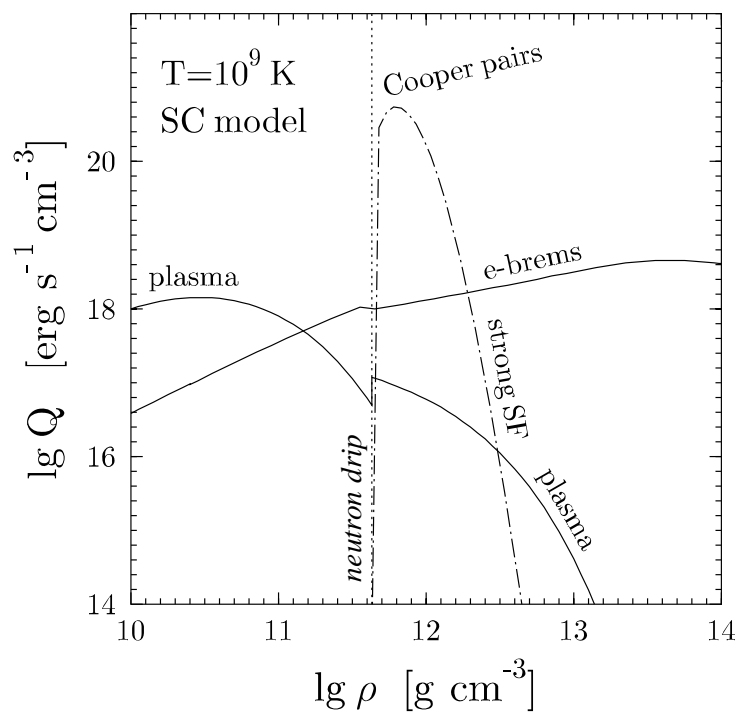

Figure 64: Neutrino emissivity from the Cooper pair formation mechanism, calculated for strong (uniform) neutron superfluidity with the maximum critical temperature $T_{\mathrm{c} n}^{\max }=1.6 \times 10^{10} \mathrm{~K}$ (a model from [231]). For comparison, $Q_{\nu}$ from two most efficient other mechanisms, plasmon decay and electron Bremsstrahlung, are also plotted. The smooth composition ground-state crust model of Kaminker et al. [231] is used; it predicts a specific $\rho_{n}(\rho)$ in the inner crust. This crust model is described in detail in Appendix B.2 of Haensel et al. [184]). From [428].

case presented in Figure $64, T_{\mathrm{cn}}^{\max }=1.6 \times 10^{10} \mathrm{~K}$ is significantly larger than $T$. The Cooperpair mechanism is efficient only within a narrow range of temperature below $T_{\mathrm{cn}}$, namely for $0.7 \lesssim T / T_{\mathrm{c} n}<1$, and is strongly damped outside this region. In view of this, $Q_{\nu}^{\mathrm{CP}}$ usually has two maxima, around $\rho_{1}$ and $\rho_{2}$, which are the two solutions of $T_{c n}(\rho)=T$ (remembering the bell shape of the pairing gap as a function of density). Only the lower-density maximum can be seen in Figure 64. Because $T_{\mathrm{c} n}^{\max } \gg T$, the localization of the peaks (at $\sim 10^{12} \mathrm{~g} \mathrm{~cm}^{-3}$ and at $\sim 10^{14} \mathrm{~g} \mathrm{~cm}^{-3}$ ) does not change much with decreasing temperature. However, the heights of the peaks decrease very fast. For the selected superfluidity model, and at $T=10^{9} \mathrm{~K}, Q_{\nu}^{\mathrm{CP}}$ in the peak region dominates over all other neutrino emission mechanisms. Let us notice that a proper inclusion of the in-medium modification of the weak interactions could significantly decrease the maximum value of $Q_{\nu}^{\mathrm{CP}}$ by about two orders of magnitude [368, 245]. 


\section{Observational Constraints on Neutron Star Crusts}

\subsection{Supernovae and the physics of hot dense inhomogeneous matter}

The stellar evolution of massive stars with a mass $M \sim 10-20 M_{\odot}$ ends with the catastrophic gravitational collapse of the degenerate iron core (for a recent review, see, for instance, [219] and references therein). Photodissociation of iron nuclei and electron captures lead to the neutronization of matter. As a result, the internal pressure resisting the gravitational pull drops, thus accelerating the collapse, which proceeds on a time scale of $\sim 0.1 \mathrm{~s}$. When the matter density inside the core reaches $\sim 10^{12} \mathrm{~g} \mathrm{~cm}^{-3}$, neutrinos become temporarily trapped, thus hindering electron captures and providing additional pressure to resist gravity. However, this is not sufficient to halt the collapse and the core contraction proceeds until the central density reaches about twice the saturation density $\rho \simeq 2.8 \times 10^{14} \mathrm{~g} \mathrm{~cm}^{-3}$ inside atomic nuclei. After that, due to the stiffness (incompressibility) of nuclear matter, the collapse halts and the core bounces, generating a shock wave. The shock wave propagates outwards against the infalling material and eventually ejects the outer layers of the star, thus spreading heavy elements into the interstellar medium. A huge amount of energy, $\sim 10^{53} \mathrm{erg}$, is released, almost entirely $(99 \%)$ in the form of neutrinos and antineutrinos of all flavors. The remaining energy is lost into electromagnetic and gravitational radiation. This scenario of core-collapse supernova explosion proved to be consistent with dense matter theory and various observations of the supernova 1987A in the Large Magellanic Cloud (discovered on February 23, 1987). In particular, the observation of the neutrino outburst provided the first direct estimate of the binding energy of the newly-born neutron star. With the considerable improvement of neutrino detectors and the development of gravitational wave interferometers, future observations of galactic supernova explosions would bring much more restrictive constraints onto theoretical models of dense matter. Supernova observations would indirectly improve our knowledge of neutron star crusts despite very different conditions, since in collapsing stellar cores and neutron star crusts the constituents are the same and are therefore described by the same microscopic Hamiltonian.

In spite of intense theoretical efforts, numerical simulations of supernovae still fail to reproduce the stellar explosion, which probably means that some physics is missing and more realistic physics input is required [280]. One of the basic ingredients required by supernova simulations is the equation of state of hot dense matter for both the inhomogeneous and homogeneous phases, up to a few times nuclear saturation densities (Section 5.4). The equation of states (EoS) plays an important role in core collapse, the formation of the shock and its propagation [402, 219]. The key parameter for the stability of the star is the adiabatic index defined by Equation (82). The stellar core becomes unstable to collapse when the pressure-averaged value of the adiabatic index inside the core falls below some critical threshold $\gamma_{c}$. A stability analysis in Newtonian gravitation shows that $\gamma_{c}=4 / 3$. The effects of general relativity increase the critical value above $4 / 3$. The precise value of the adiabatic index in the collapsing core depends on the structure and composition of the hot dense matter and, in particular, on the presence of nuclear pastas, as can be seen in Figure 36. The composition of the collapsing core and its evolution into a proto-neutron star depend significantly on the EoS. The mass fractions of the various components present inside the stellar core during the collapse are shown in Figure 65 for two different EoS, the standard Lattimer \& Swesty [255] EoS (L\&S) based on a compressible liquid drop model and the recent relativistic mean field EoS of Shen et al. [374, 375] (note however that the treatment of the inhomogeneous phases is not quantal but is based on the semi-classical Thomas-Fermi approximation, discussed in Section 3.2.2). As seen in Figure 65, the L\&S EoS predicts a larger abundance of free protons than the Shen EoS. As a consequence, the L\&S EoS enhances electron captures compared to the Shen EoS and leads to a stronger deleptonization of the core, thus affecting the formation of a shock wave. The effects of the EoS are more visible during the late period of the propagation of 
the shock wave as shown in Figure 66. The L\&S EoS leads to a more compact proto-neutron star, which is therefore hotter and has higher neutrino luminosity, as can be seen in Figure 67.

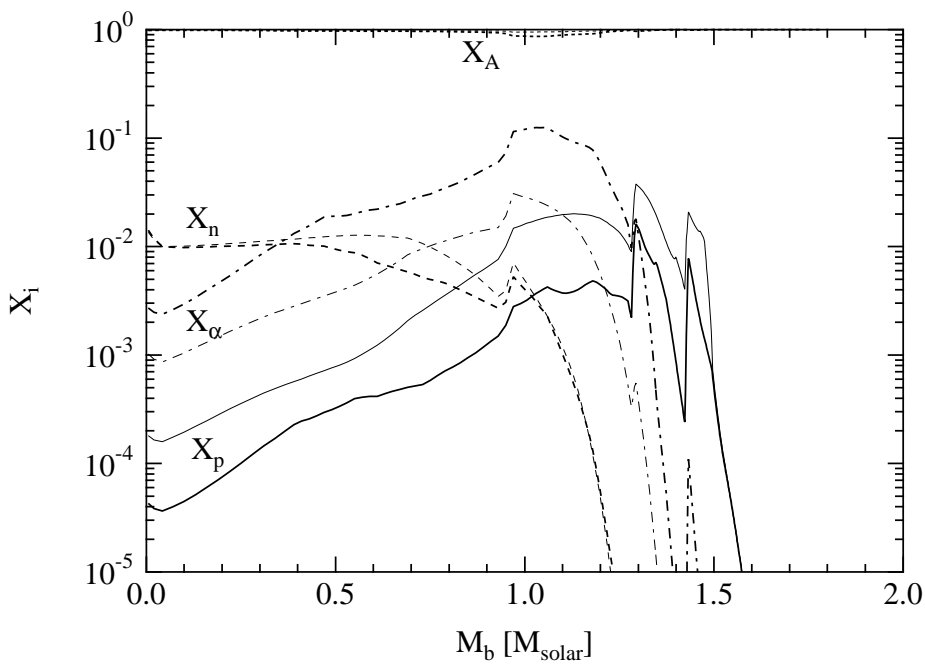

Figure 65: Mass fractions of different particles in a supernova core as a function of baryon mass coordinate at the time when the central density reaches $10^{11} \mathrm{~g} \mathrm{~cm}^{-3}$. Solid, dashed, dotted, and dot-dashed lines show mass fractions of protons, neutrons, nuclei, and alpha particles, respectively. The results are given for two equations of state: the compressible liquid drop model of Lattimer \& Swesty [255] (thin lines) and the relativistic mean field theory in the local density approximation of Shen et al. [374, 375] (thick lines). See [402] for details.

The collapse of the supernova core and the formation of the proto-neutron star are governed by weak interaction processes and neutrino transport [250]. Numerical simulations generally show that as the shock wave propagates outwards, it loses energy due to the dissociation of heavy elements and due to the pressure of the infalling material so that it finally stalls around $\sim 10^{2} \mathrm{~km}$, as can be seen, for instance, in Figure 66. According to the delayed neutrino-heating mechanism, it is believed that the stalled shock is revived after $\sim 100 \mathrm{~ms}$ by neutrinos, which deposit energy in the layers behind the shock front. The interaction of neutrinos with matter is therefore crucial for modeling supernova explosions. The microscopic structure of the supernova core has a strong influence on the neutrino opacity and, therefore, on the neutrino diffusion timescale. In the relevant core layers, neutrinos form a nondegenerate gas, with a de Broglie wavelength $\lambda_{\nu}=2 \pi \hbar c / E_{\nu}$, where $E_{\nu} \sim 3 k_{\mathrm{B}} T \sim 5-10 \mathrm{MeV}$. If $\lambda_{\nu}>2 R_{A}$, where $R_{A}$ is the radius of a spherical cluster, then thermal neutrinos "do not see" the individual nucleons inside the cluster and scatter coherently on the $A$ nucleons. Putting it differently, a neutrino couples to a single weak current of the cluster of $A$ nucleons. If the neutrino scattering amplitude on a single nucleon is $f$, then the scattering amplitude on a cluster is $A f$, and the scattering cross section is $\sigma_{A}^{\text {coh }}=A^{2}|f|^{2}$ ([150], for a review, see [373]). Consider now the opposite case of $\lambda_{\nu} \ll 2 R_{A}$. Neutrinos scatter on every nucleon inside the cluster. As a result, the scattering amplitudes add incoherently, and the neutrino-nucleus scattering cross section $\sigma_{A}^{\text {incoh }}=A|f|^{2}$, similar to that for a gas of $A$ nucleons. In this way, $\sigma_{A}^{\text {coh }} / \sigma_{A}^{\text {incoh }} \approx A \sim 100$. One therefore concludes, that the presence of clusters in hot matter can dramatically increase the neutrino opacity. The neutrino transport in supernova cores depends not only on the characteristic size of the clusters, but also on their geometrical shape and topology. In particular, the presence of an heterogeneous plasma (due to thermal statistical distribution of $A$ and $Z$ ) in the supernova core [65] or the existence of nuclear pastas instead of spherical

Living Reviews in Relativity

http://www. livingreviews.org/Irr-2008-10 


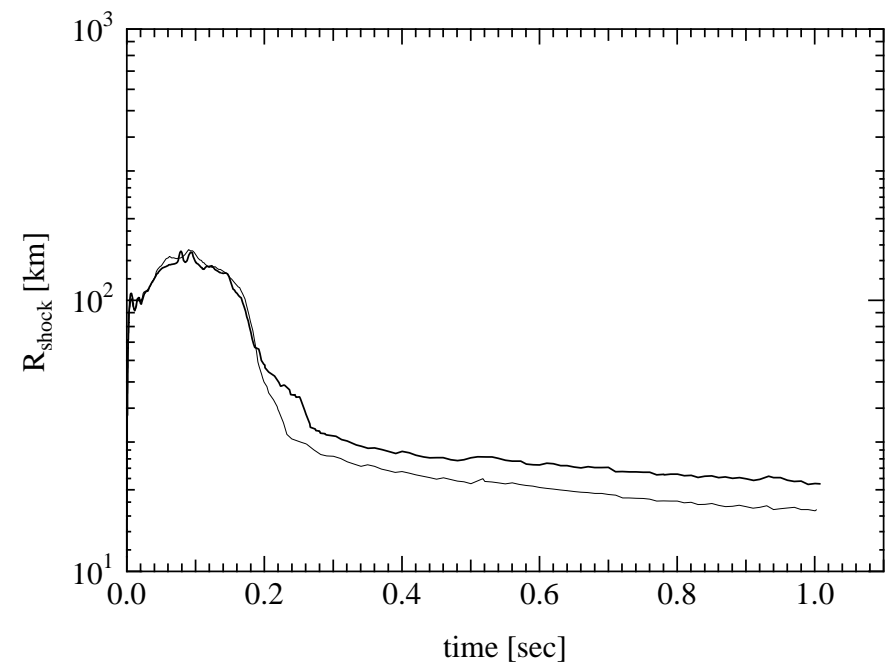

Figure 66: Radial positions of shock waves as a function of time after bounce (the moment of greatest compression of the central core corresponding to a maximum central density) for two different equations of states: the compressible liquid drop model of Lattimer \& Swesty [255] (thin line) and the relativistic mean field theory in the local density approximation of Shen et al. [374, 375] (thick line). See [402] for details. Notice that these particular models failed to produce a supernova explosion.

clusters [201, 384] have a sizeable effect on the neutrino propagation. The outcome is that the neutrino opacity of inhomogeneous matter is considerably increased compared to that of uniform matter.

\subsection{Cooling of isolated neutron stars}

Neutron stars are born in the core collapse supernova explosions of massive stars, as briefly reviewed in Section 12.1. During the first tens of seconds, the newly formed proto-neutron star with a radius of $\sim 50 \mathrm{~km}$ stays very hot with temperatures on the order of $10^{11}-10^{12} \mathrm{~K}$. In the following stage, the star becomes transparent to neutrinos generated in its interior via various processes (see Section 11). Within $\sim 10-20 \mathrm{~s}$ the proto-neutron star thus rapidly cools down by powerful neutrino emission and shrinks into an ordinary neutron star. The last cooling stage, after about $10^{4}-10^{5}$ years, is governed by the emission of thermal photons due to the diffusion of heat from the interior to the surface (for a recent review of neutron star cooling, see, for instance, [431, 318] and references therein). Neutron stars in X-ray binaries may be heated as a result of the accretion of matter from the companion star. Observational data and references have been collected on the UNAM webpage [212].

The cooling of a young neutron star is very sensitive to its crust physics including, for example, neutron superfluidity, as shown in Figure 68. Superfluidity of free neutrons in the inner crust suppresses heat capacity. Moreover, superfluidity opens a new channel for neutrino emission. Indeed, the formation of a bound neutron pair liberates energy, which can be converted into a neutrino-antineutrino pair, as discussed in Section 11.6.

\subsubsection{Thermal relaxation of the crust}

Due to its relatively low neutrino emissivity, the crust of a newly-born neutron star cools less rapidly than the core and thus stays hotter. As a result, the surface temperature decreases slowly 

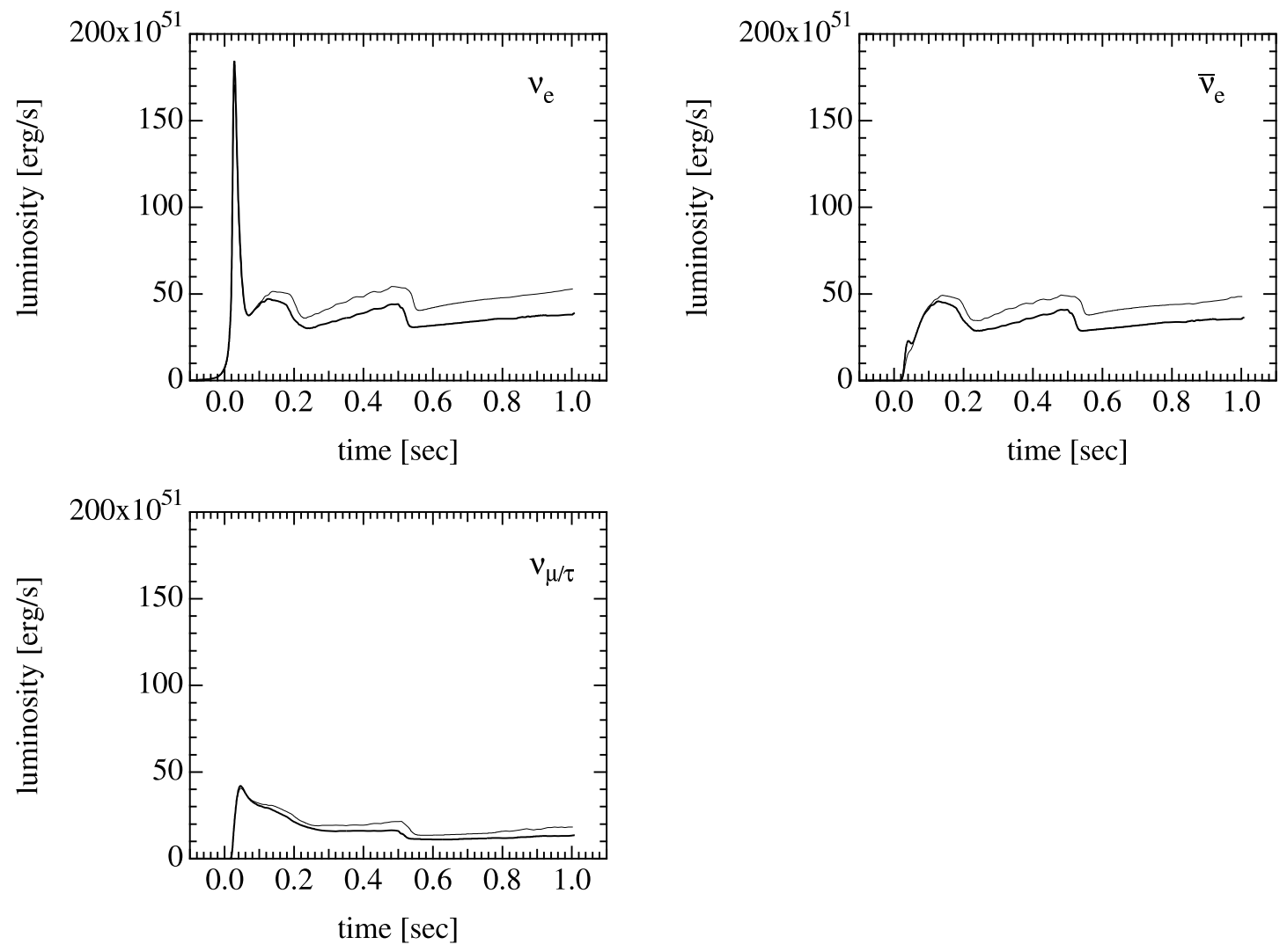

Figure 67: Luminosities of $\nu_{e}, \bar{\nu}_{e}$, and $\nu_{\mu / \tau}$ as a function of time after bounce (the moment of greatest compression of the central core corresponding to a maximum central density) for two different equations of state: the compressible liquid drop model of Lattimer \& Swesty [255] (thin lines) and the relativistic mean field theory in the local density approximation of Shen et al. [374, 375] (thick lines). See [402] for details. 


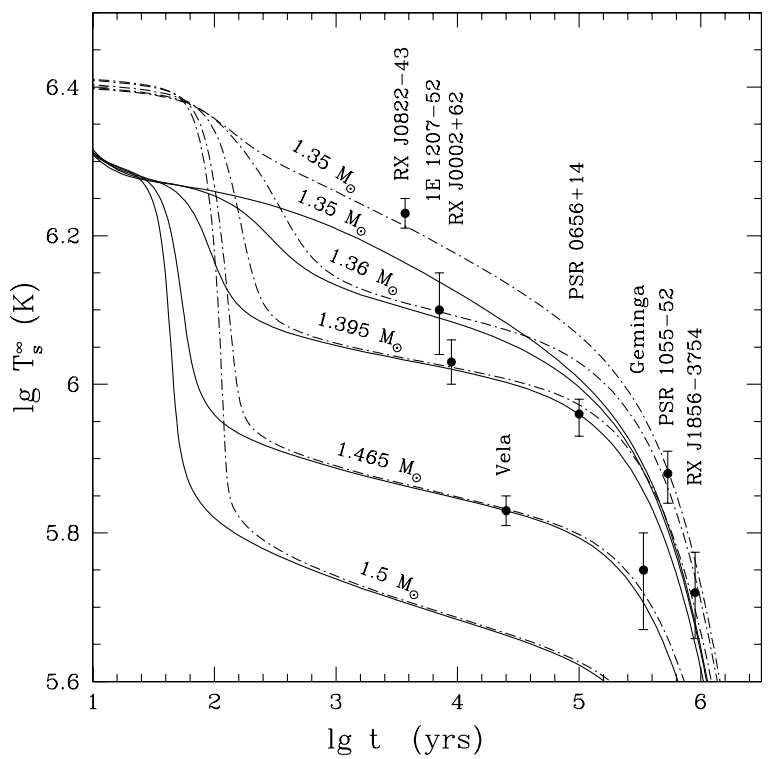

Figure 68: Redshifted surface temperatures (as seen by an observer at infinity) vs. age of neutron stars with different masses as compared with observation. Dot-dashed curves are calculated with only proton superfluidity in the core. Solid curves also include neutron superfluidity in the crust and outer core [428].

during the first ten to hundred years and then drops sharply when the cooling wave from the core reaches the surface as illustrated in Figure 68. After time $t_{w}$, the star becomes isothermal except for the very outer layers. The relaxation time $t_{w}$ of reaching a quasi-isothermal state depends, in particular, on the specific heat $C_{v}$ and on the thermal conductivity $\kappa$ of the inner crust (see Section 9.3) and is approximately given by $[256,167]$

$$
t_{w} \sim(\Delta R)^{2}\left(1-\frac{r_{\mathrm{g}}}{R}\right)^{-3 / 2} \frac{C_{\mathrm{v}}}{\kappa},
$$

where $\Delta R$ is the thickness of the crust, $R$ the circumferential radius of the star and $r_{\mathrm{g}}=2 G M / c^{2}$, the Schwarzschild radius. The ratio of specific heat $C_{\mathrm{v}}$ to thermal conductivity $\kappa$ has to be taken at half nuclear saturation density, slightly lower than the crust bottom density $\rho_{\text {cc }}$ (see [428]; in general, the relaxation time is the most sensitive to $\kappa$ and $C_{\mathrm{v}}$ in the density range $0.1 \rho_{\mathrm{cc}}<\rho<\rho_{\mathrm{cc}}$ ). The thermal conductivity of the crust comes mainly from electrons scattering off atomic nuclei and electrically charged impurities. It is crucially dependent on the structure and composition of the crust (see Section 9). The crustal specific heat is dominated by free neutrons if they are not superfluid. Otherwise the neutron specific heat is strongly suppressed and its contribution to the total heat capacity is negligible as can be seen in Figure 69. However, the density range and the critical temperatures for neutron superfluidity in the crust are still not very well known. The presence of nuclear inhomogeneities can have a significant effect on the specific heat by reducing the neutron pairing correlations inside the nuclei especially in the shallow layers of the inner crust at densities $\rho \sim 10^{11}-10^{12} \mathrm{~g} \mathrm{~cm}^{-3}[332,361,237,294]$. The cooling curves of a $1.5 M_{\odot}$ neutron star for different crust models are shown in Figure 70. Observations of young neutron stars could thus put constraints on the thermal properties of the crust, which in turn depend on its structure and composition. Such young neutron stars have not been observed yet. One reason might be that neutron stars born in type II supernova explosions remain hidden by the expanding supernova envelopes for many years. 


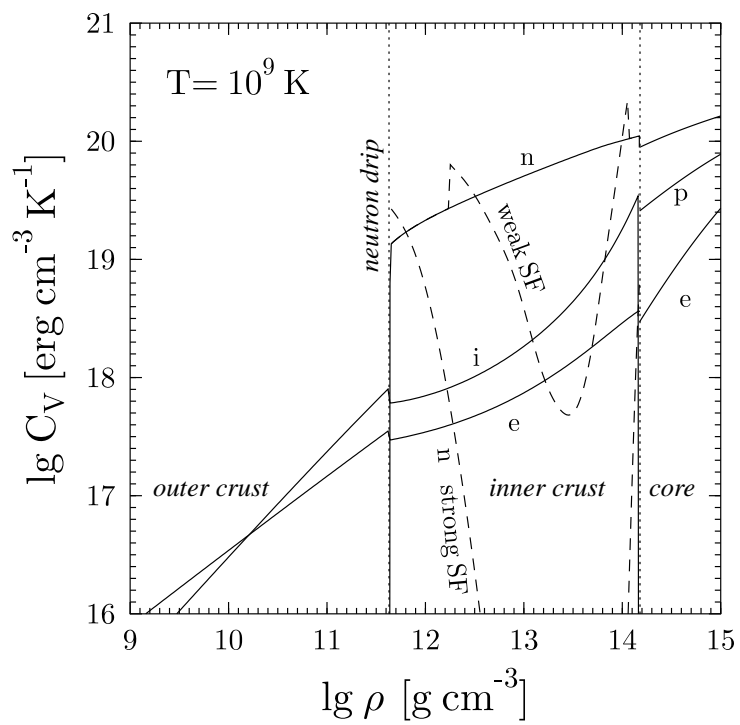

Figure 69: Neutron star specific heat at $T=10^{9} \mathrm{~K}$. Solid lines: partial heat capacities of ions (i), electrons (e) and free neutrons (n) in nonsuperfluid crusts, as well as of neutrons, protons (p) and electrons in nonsuperfluid cores. Dashed lines: heat capacities of free neutrons in the crust modified by superfluidity. Two particular models of weak and strong superfluidity are considered. The effects of the nuclear inhomogeneities on the free neutrons are neglected. From [167].

\subsubsection{Observational constraints from thermal X-ray emission}

In cooling simulations, the neutron star is usually decomposed into the stellar interior, which becomes isothermal after a few tens to hundreds of years and the outer heat blanketing (insulating) envelope, where temperature gradients persist due to low thermal conductivity. The boundary between the interior and the envelope is conventionally set at $\rho=10^{10} \mathrm{~g} \mathrm{~cm}^{-3}$. The relationship between the surface temperature $T_{\mathrm{s}}$ and the temperature $T_{\mathrm{b}}$ at the bottom of the heat blanketing envelope is very sensitive to the structure and the composition of the crust and to the presence of a magnetic field. The outermost envelope of a neutron star, composed mainly of iron (Section 3.1), may be covered by a thin layer of light elements due to accretion, which strongly enhances heat transport and increases the surface temperature for a given $T_{\mathrm{b}}$ (let us remember that the electron thermal conductivity in a Coulomb plasma of ions with charge $Z$ varies as $\sim 1 / Z$ ). Strong magnetic fields also affect heat transport, leading to a nonuniform surface-temperature distribution and, in particular, hot caps near the magnetic poles, as illustrated in Figure 71.

The effects of different crust models on the relationship between the surface temperature $T_{\mathrm{s}}$ and the temperature $T_{\mathrm{b}}$ at the bottom of the heat-blanketing envelope, are illustrated in Figure 72. Grigorian [176] recently argued that cooling models predicting neutron stars with an age between about $10^{3}-10^{4}$ years to be hotter than those already observed, should be rejected since if such stars existed in our galaxy, they would have already been detected. This brightness constraint puts restrictions on the $T_{\mathrm{s}}-T_{\mathrm{b}}$ relationship hence on crust models.

\section{3 r-process in the decompression of cold neutron star crusts}

The location of the astrophysical site for the rapid neutron capture process (r-process), thought to be responsible for the production of many heavy neutron-rich nuclei with $A>60$ in the universe, still remains uncertain (for a recent review, see, for instance, [23]). Many possible sites have

Living Reviews in Relativity

http://www. livingreviews . org//rr-2008-10 


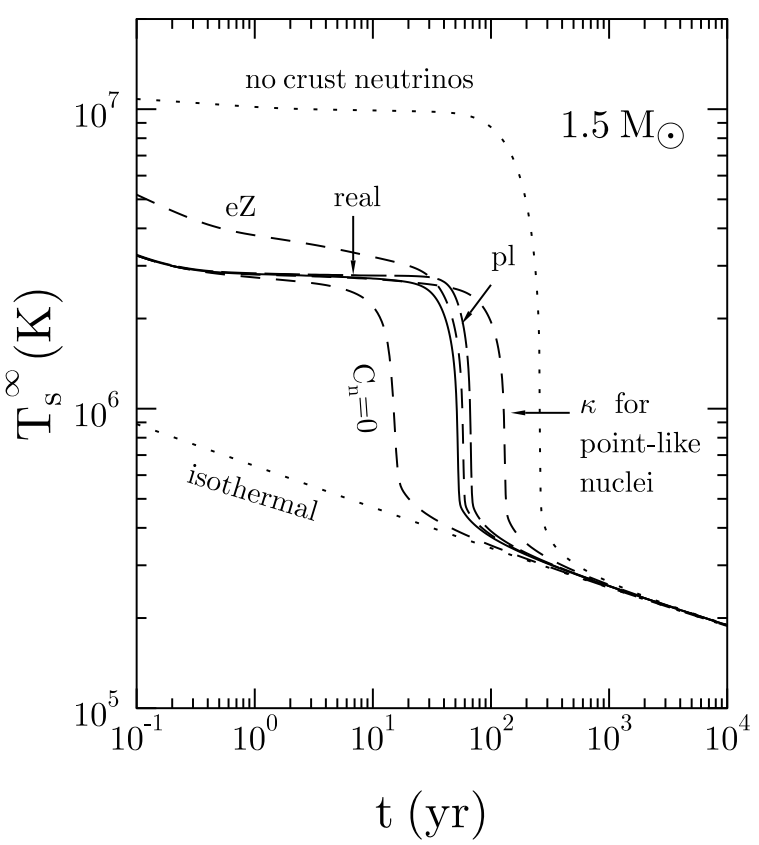

Figure 70: Effective surface temperature (as seen by an observer at infinity) of a $1.5 M_{\odot}$ neutron star during the first hundred years for different crust models. Dotted lines: cooling without neutrino emission from the crust (upper line), infinite $\kappa$ at $\rho>10^{10} \mathrm{~g} \mathrm{~cm}^{-3}$. Solid line: cooling curve for the best values of $\kappa, C_{\mathrm{v}}$, and $Q_{\nu}$. Dashed line $C_{n}=0$ : dripped neutrons heat capacity removed. Dashed curve $\kappa$ : thermal conductivity calculated assuming point-like nuclei. Two other dashed lines: neutrino emission processes removed except for plasmon decay $(\mathrm{pl})$ or electron-nucleus Bremsstrahlung $(e Z)$. See also line $1.5 M_{\odot}$ in Table 2 of [167].
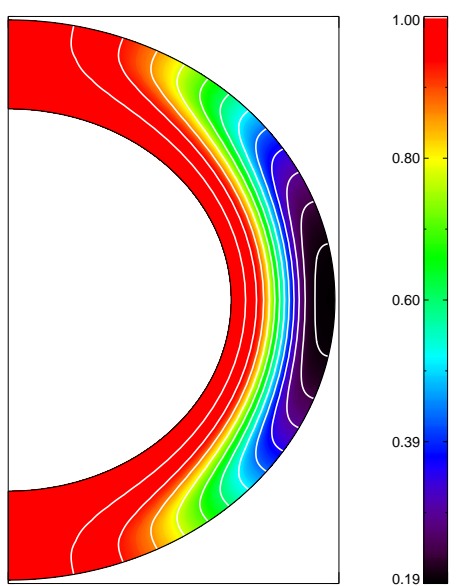

Figure 71: Magnetic field lines and temperature distribution in a neutron star crust for an axisymmetric dipolar magnetic field $B=3 \times 10^{12} \mathrm{G}$ and an isothermal core with temperature $T_{\text {core }}=10^{6} \mathrm{~K}$. The temperature is measured in units of $T_{\text {core }}$. The magnetic field is confined to the crust. From [158]. 


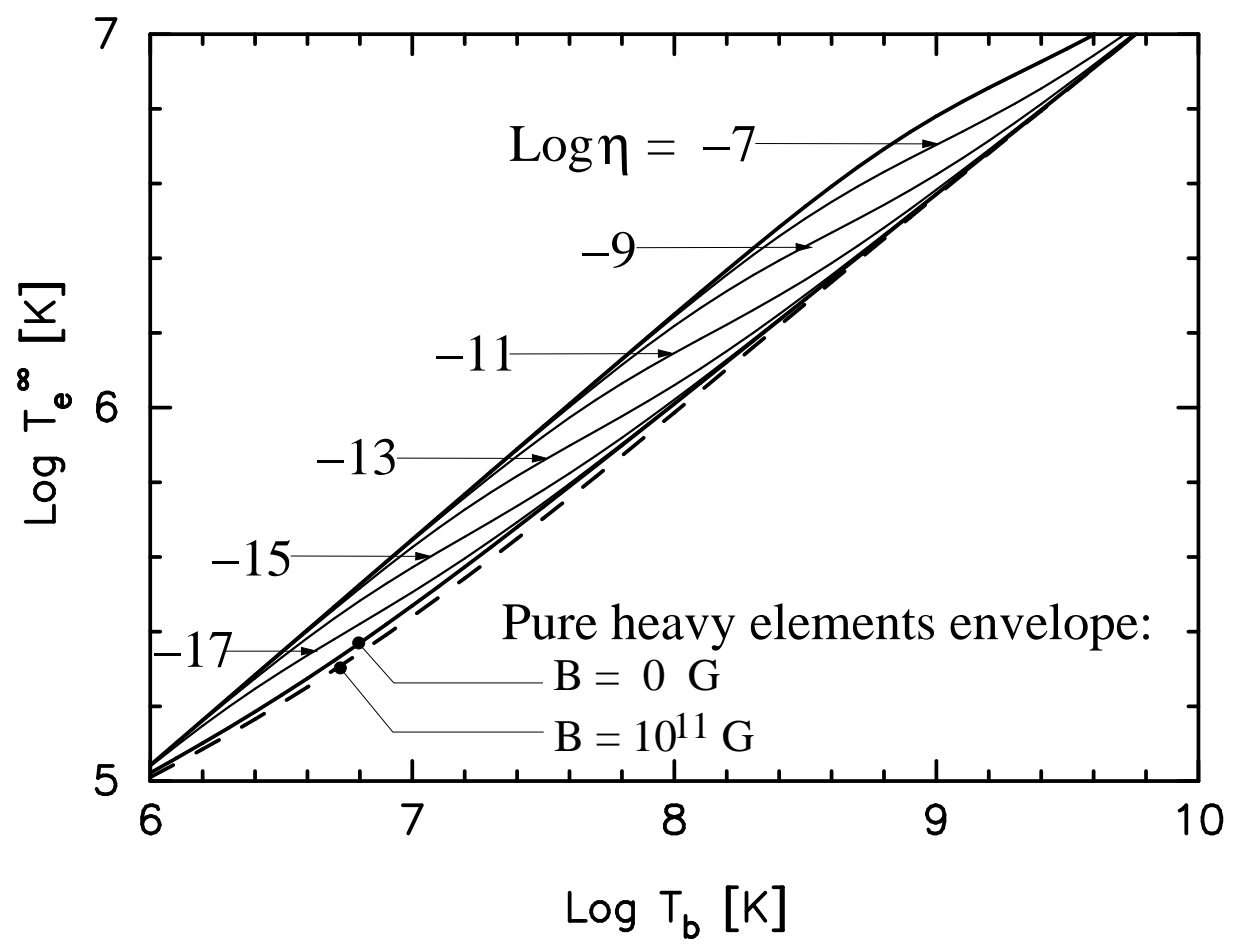

Figure 72: Relationship between the effective surface temperature $T_{\text {eff }}$, as measured by an observer at infinity, and the local temperature at the bottom of the heat-blanketing envelope, $T_{\mathrm{b}}\left(\right.$ at $\rho_{\mathrm{b}}=10^{11} \mathrm{~g} \mathrm{~cm}^{-3}$ ). Calculations performed for the ground-state $(\mathrm{Fe})$ and partly-accreted envelopes of mass $\Delta M$. Numbers $-16, \ldots,-7$ indicate $\log _{10}(\Delta M / M)$ for a $1.4 M_{\odot}$ star with surface gravity $g_{14}=2.43$ (in units of $10^{14} \mathrm{~cm} \mathrm{~s}^{-2}$ ). Symbols in parentheses indicate chemical composition of accreted envelope. 
been considered, but they all have serious problems. The most studied scenarios are related to neutrino-driven wind during type II supernova explosions or $\gamma$-ray bursts. Nevertheless, apart from many uncertainties in the explosion mechanism, the conditions for the r-process to occur are difficult to reach and require a fine tuning of model parameters. Lattimer et al. [253] suggested a long time ago that the r-process could also occur during the decompression of cold crustal matter ejected into the interstellar medium. This possibility has remained largely unexplored until very recently (see [170, 23] and references therein). This scenario is, however, promising because the presence of neutron-rich nuclei, the large neutron-to-seed ratio and the low electron fraction in the decompressing crustal matter are favorable conditions for the r-process to occur. Various scenarios can be envisioned. Matter could be ejected into the interstellar medium by outflows from newlyborn proto-neutron stars or jets such as those recently observed in Circinus X-1 [194]. Neutron stars very rapidly spinning beyond the mass-shedding limit would also expel matter. In the early years of pulsar astronomy, Dyson [131] suggested that neutron stars might have volcanic activity. This idea of cataclysmic events has been more recently revived by the observations of giant flares in magnetars, thought to be the signature of magnetic crustquakes. From observations of the radio afterglow [156] it has been estimated that more than $10^{-9} M_{\odot}$ was ejected during the December 27, 2004 event in SGR 1806-20. More exotic astrophysical events have been proposed, such as the explosion of a neutron star below the minimum mass [401] or the phase transition into a strange quark star (quark-novae) [218]. However the merging of a neutron star and a black hole or of two neutron stars (see [337] for a recent review on compact binaries) is probably the most likely scenario for the ejection of large amounts of matter. This tidal disruption of two merging neutron stars has recently been studied in detail $[170,23]$, motivated by the results of hydrodynamic simulations, which show that up to $10^{-2} M_{\odot}$ could be ejected in this manner. This study has proven that the solar system abundance pattern can be qualitatively reproduced by considering the decompression of clumps of neutron-star-crust matter with different initial densities, as shown in Figure 73.

\subsection{Pulsar glitches}

Since the discovery by Jocelyn Bell and Anthony Hewish in 1967 of highly-periodic radio sources soon identified with rotating neutron stars (Hewish was awarded the Nobel Prize in Physics in 1974 [305]), more than 1700 pulsars have been found at the time of writing (pulsar timing data are available online at [26]). Pulsars are the most precise clocks with rotation periods ranging from about 1.396 milliseconds for the recently discovered pulsar J1748-2446ad [195] up to several seconds. The periodicity of arrival time of pulses is extremely stable. The slight delays associated with the spin-down of the star are at most of a few tens of microseconds per year. Nevertheless, longterm monitoring of pulsars has revealed irregularities in their rotational frequencies.

The first kind of irregularity, called timing noise, is random fluctuations of pulse arrival times and is present mainly in young pulsars, such as the Crab, for which the slow down rate is larger than for older pulsars. Indeed, correlations have been found between the spin-down rate and the noise amplitude [275]. Timing noise might result from irregular transfers of angular momentum between the crust and the liquid interior of neutron stars. A second kind of irregularity is the sudden jumps or "glitches" of the rotational frequency, which have been observed in radio pulsars and more recently in anomalous X-ray pulsars [234, 232, 106, 233, 296]. An example of a glitch is shown in Figure 74. Evidence of glitches have also been reported in accreting neutron stars [153]. These glitches, whose amplitude vary from $\Delta \Omega / \Omega \sim 10^{-9}$ up to $\Delta \Omega / \Omega \sim 16 \times 10^{-6}$ for PSR J1806-2125 [199], as shown in Figure 75, are followed by a relaxation over days to years and are sometimes accompanied by a sudden change of the spin-down rate from $|\Delta \dot{\Omega} / \dot{\Omega}| \sim 10^{-6}-10^{-5}$ to $|\Delta \dot{\Omega} / \dot{\Omega}| \sim 10^{-3}-10^{-2}$. By the time of this writing, 171 glitches have been observed in 50 pulsars. Their characteristics and the references can be found at [24]. The time between two successive glitches is usually a few years. One of the most active pulsars is PSR J1341-6220, for 


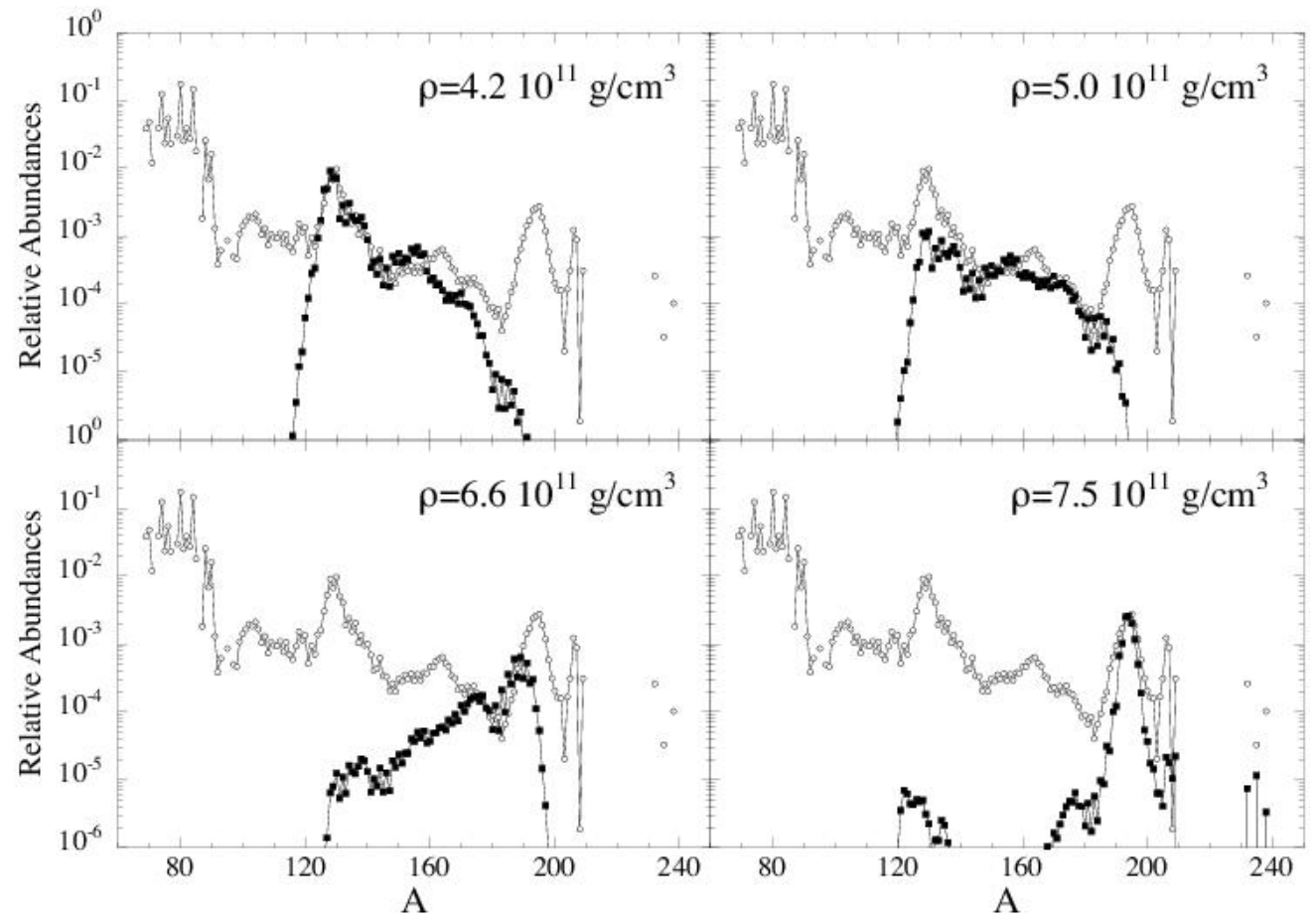

Figure 73: Final composition of clumps of ejected neutron star crust with different initial densities (solid squares). The open circles correspond to the solar system abundance of r-elements. From [23].

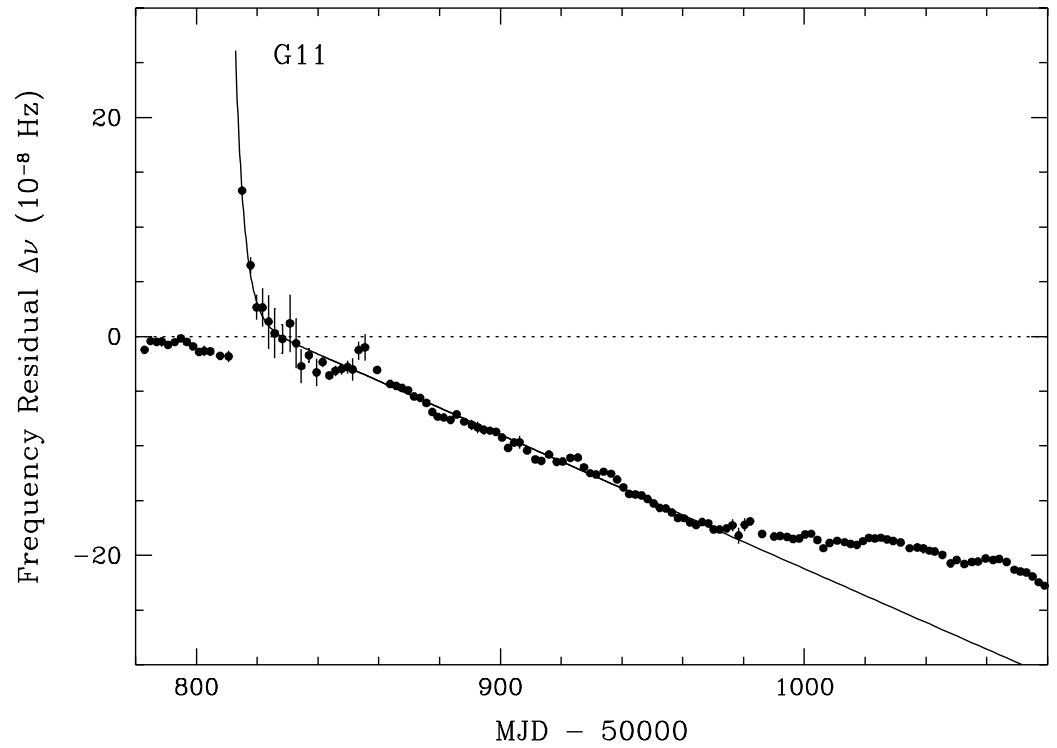

Figure 74: Glitch $\Delta \Omega / \Omega \sim 9 \times 10^{-9}$ observed in the Crab pulsar by Wong et al. [425] 
which 12 glitches have been detected during 8.2 years of observation [416].

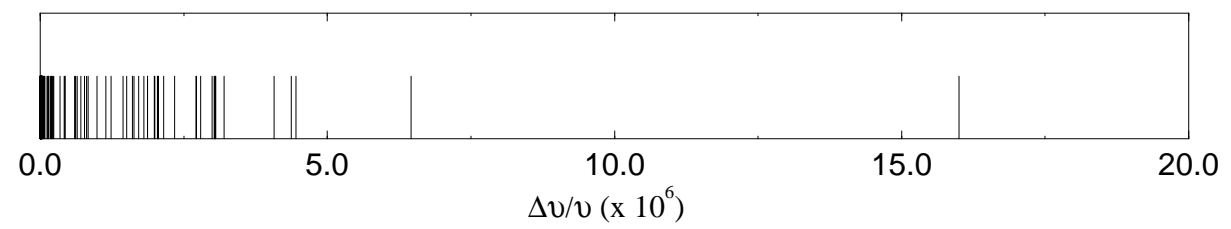

Figure 75: Amplitudes of 97 pulsar glitches, including the very large glitch $\Delta \Omega / \Omega \sim 16 \times 10^{-6}$ observed in PSR J1806-2125 [199].

Very soon after the observations of the first glitches in the Crab and Vela pulsars, superfluidity in the interior of neutron stars was invoked to explain the long relaxation times [41]. The possibility that dense nuclear matter becomes superfluid at low temperatures was suggested theoretically much earlier, even before the discovery of the first pulsars (see Section 8.2). Following the first observations, several scenarios were proposed to explain the origin of these glitches, such as magnetospheric instabilities, pulsar disturbance by a planet, hydrodynamic instabilities or collisions of infalling massive objects (for a review of these early models, see Ruderman [350]). Most of these models had serious problems. The most convincing interpretation was that of starquakes, as briefly reviewed in Section 12.4.1. However, large amplitude glitches remained difficult to explain. The possible role of superfluidity in pulsar glitches was first envisioned by Packard in 1972 [317]. Soon after, Anderson and Itoh proposed a model of glitches based on the motion of neutron superfluid vortices in the crust [10]. Laboratory experiments were carried out to study similar phenomena in superfluid helium $[68,408,409]$. It is now widely accepted that neutron superfluidity plays a major role in pulsar glitches. As discussed in Sections 12.4.2 and 12.4.3, the glitch phenomenon seems to involve at least two components inside neutron stars: the crust and the neutron superfluid. Section 12.4.4 shows how the observations of pulsar glitches can put constraints on the structure of neutron stars.

\subsubsection{Starquake model}

Soon after the observations of the first glitches in Vela and in the Crab pulsars, Ruderman [349] suggested that these events could be the manifestations of starquakes (see also [184] and references therein). As a result of centrifugal forces, rotating neutron stars are not spherical but are slightly deformed, as can be seen in Figure 40. If the star were purely fluid, a deceleration of its rotation would entail a readjustment of the stellar shape to a more spherical configuration. However, a solid crust prevents such readjustment and consequently the star remains more oblate. The spindown of the star thus builds up stress in the crust. When this stress reaches a critical level, the crust cracks and the star readjusts its shape to reduce its deformation. Assuming that the angular momentum is conserved during a starquake, the decrease $\Delta I<0$ of the moment of inertia $I$ is therefore accompanied by an increase $\Delta \Omega>0$ of the rotational frequency $\Omega$ according to

$$
\frac{\Delta \Omega}{\Omega}=-\frac{\Delta I}{I} .
$$

For a purely fluid rotating star, the moment of inertia can be written as

$$
I=I_{0}(1+\epsilon)
$$


where $I_{0}$ is the moment of inertia of a nonrotating spherical star. The parameter $\epsilon$ is proportional to $\Omega^{2}$ and is typically very small. For instance, for the Crab and Vela pulsars, $\epsilon \sim 10^{-4}$ and $10^{-5}$, respectively. Since the decrease of the moment of inertia of a pulsar can be at most equal to that of a purely fluid star, this model predicts that the glitch amplitude is

$$
\frac{\Delta \Omega}{\Omega}<\epsilon \frac{\delta t}{\tau}
$$

where $\tau=\dot{\Omega} / 2 \Omega$ and $\delta t$ is the time between two successive starquakes (on the order of years for the Vela and Crab pulsars). This model is consistent with the glitches observed in the Crab pulsar and explains the weak glitch activity of young pulsars by the fact that the internal temperatures are still too high for the crust to store a large stress. However, for the Vela pulsar, with $\tau \sim 10^{4}$ years and $\epsilon \sim 10^{-5}$, this model predicts glitch amplitude of $\Delta \Omega / \Omega \sim 10^{-9}$, about three orders of magnitude smaller than those observed. The starquake model fails to explain all the observations of pulsar glitches. Therefore, other mechanisms have to be invoked.

\subsubsection{Two-component models}

Due to the interior magnetic field, the plasmas of electrically charged particles inside neutron stars are strongly coupled and co-rotate with the crust on very long timescales on the order of the pulsar age [132], thus following the long-term spin-down of the star caused by the electromagnetic radiation. Besides, the crust and charged particles are rotating at the observed angular velocity of the pulsar due to coupling with the magnetosphere. In contrast, neutrons are electrically neutral and superfluid. As a consequence, they can rotate at a different rate by forming quantized vortex lines (Section 8.3.2). This naturally leads to the consideration of the stellar interior as a two-fluid mixture. A model of this kind was first suggested by Baym et al. [40] for interpreting pulsar glitches as a transfer of angular momentum between the two components. Following a sudden spin-up of the star after a glitch event, the plasma of charged particles readjusts to a new rotational frequency within a few seconds [133]. Moreover, as discussed in Section 8.3.7, neutron superfluid vortices carry magnetic flux giving rise to an effective mutual friction force acting on the superfluid. As a result, the neutron superfluid in the core is dynamically coupled to the crust and to the charged particles, on a time scale much shorter than the post-glitch relaxation time of months to years observed in pulsars like Vela, suggesting that glitches are associated with the neutron superfluid in the crust. This conclusion assumes that the distribution of proton flux tubes in the liquid core is uniform. Nevertheless, one model predicts that every neutron vortex line is surrounded by a cluster of proton flux tubes [369, 370]. In this vortex-cluster model, the coupling time between the core superfluid and the crust could be much longer than the previous estimates and could be comparable to the postglitch relaxation times.

The origin of pulsar glitches relies on a sudden release of stresses accumulated in the crust, similar to the starquake model. However, the transfer of angular momentum from the rapidlyrotating neutron superfluid to the magnetically-braked solid crust and charged constituents during a glitch allows much larger spin-up than that due solely to the readjustment of the stellar shape. Neutron superfluid is weakly coupled to a normal charged component by mutual friction forces and thus follows the spin-down of the crust via a radial motion of the vortices away from the rotation axis unless the vortices are pinned to the crust. In the latter case, the lag between the superfluid and the crust induces a Magnus force, acting on the vortices producing a crustal stress. When the lag exceeds a critical threshold, the vortices are suddenly unpinned. Vortex motion could also be initiated by a temperature perturbation, for instance the heat released after a starquake [267]. As a result, the superfluid spins down and, by the conservation of the total angular momentum, the crust spins up leading to a glitch [10]. If the pinning is strong enough, the crust could crack before the vortices become unpinned, as suggested by Ruderman [351, 352, 356, 353]. These two scenarios

Living Reviews in Relativity

http: //www. livingreviews . org//rr-2008-10 
lead to different predictions for the internal heat released after a glitch event. It has been argued that observations of the thermal X-ray emission of glitching pulsars could thus put constraints on the glitch mechanism [251].

In the vortex creep model [9] a postglitch relaxation is interpreted as a motion of vortices due to thermal fluctuations. Even at zero temperature, vortices can become unpinned by quantum tunneling [268]. The vortex current increases with the growth of temperature and can prevent the accumulation of large crustal stress in young pulsars, thus explaining the low glitch activity of these pulsars. In the model of Alpar et al. [6, 7] a neutron star is analogous to an electric circuit with a capacitor and a resistor, the vortices playing the role of the electric charge carriers. The star is, thus, assumed to be formed of resistive regions, containing a continuous vortex current, and capacitive regions devoid of vortices. A glitch can then be viewed as a vortex "discharge" between resistive regions through capacitive regions. The permanent change in the spin-down rate observed in some pulsars is interpreted as a reduction of the moment of inertia due to the formation of new capacitive regions. A major difficulty of this model is to describe the unpinning and repinning of vortices.

Ruderman developed an alternative view based on the interactions between neutron vortices and proton flux tubes in the core, assuming that the protons form a type II superconductor [354, 355]. Unlike the vortex lines, which are essentially parallel to the rotation axis, the configuration of the flux tubes depends on the magnetic field and may be quite complicated. Recalling that the number of flux tubes per vortex is about $10^{13}$ (see Sections 8.3.3 and 8.3.4), it is therefore likely that neutron vortices and flux tubes are strongly entangled. As superfluid spins down, the vortices move radially outward dragging along the flux tubes. The motion of the flux tubes results in the build up of stress in the crust. If vortices are strongly pinned to the crust, the stress is released by starquakes fracturing the crust into plates like the breaking of a concrete slab reinforced by steel rods when pulling on the rods. These plates and the pinned vortices will move toward the equator thus spinning down superfluid and causing a glitch. Since the magnetic flux is frozen into the crust due to very high electrical conductivity, the motion of the plates will affect the configuration of the magnetic field. This mechanism naturally explains the increase of the spin-down rate after a glitch observed in some pulsars like the Crab, by an increase of the electromagnetic torque acting on the pulsar due to the increase of the angle between the magnetic axis and the rotation axis.

\subsubsection{Recent theoretical developments}

Other scenarios have recently been proposed for explaining pulsar glitches, such as transitions from a configuration of straight neutron vortices to a vortex tangle [324], and more exotic mechanisms invoking the possibility of crystalline color superconductivity of quark matter in a neutron star core [4]. These models, and those briefly reviewed in Section 12.4.2, rely on rather poorly known physics. The strength of the vortex pinning forces and the type of superconductivity in the core are controversial issues (for a recent review, see, for instance, [367] and references therein). Besides, it is usually implicitly assumed that superfluid vortices extend throughout the star (or at least throughout the inner crust). However, microscopic calculations show that the superfluidity of nuclear matter strongly depends on density (see Section 8.2). It should be remarked that even in the inner crust, the outermost and innermost layers may be nonsuperfluid, as discussed in Section 8.2.2. It is not clear how superfluid vortices arrange themselves if some regions of the star are nonsuperfluid. The same question also arises for magnetic flux tubes if protons form a type II superconductor.

Andersson and collaborators [16] have suggested that pulsar glitches might be explained by a Kelvin-Helmholtz instability between neutron superfluid and the conglomeration of charged particles, provided the coupling through entrainment (see Section 8.3.7) is sufficiently strong. It remains to be confirmed whether such large entrainment effects can occur. Carter and collaborators [81] 
pointed out a few years ago that a mere deviation from the mechanical and chemical equilibrium induced by the lack of centrifugal buoyancy is a source of crustal stress. This mechanism is always effective, independently of the vortex motion and proton superconductivity. In particular, even if the neutron vortices are not pinned to the crust, this model leads to crustal stress of similar magnitudes than those obtained in the pinned case. Chamel \& Carter [94] have recently demonstrated that the magnitude of the stress is independent of the interactions between neutron superfluid and normal crust giving rise to entrainment effects. But they have shown that stratification induces additional crustal stress. In this picture, the stress builds up until the lag between neutron superfluid and the crust reaches a critical value, at which point the crust cracks, triggering a glitch. The increase of the spin-down rate observed in some pulsars like the Crab can be explained by the crustal plate tectonics of Ruderman [351, 352, 356, 353], assuming that neutron superfluid vortices remain pinned to the crust. Even in the absence of vortex pinning, Franco et al. [149] have shown that, as a result of starquakes, the star will oscillate and precess before relaxing to a new equilibrium state, followed by an increase of the angle between the magnetic and rotation axis (thus increasing the spin-down rate).

\subsubsection{Pulsar glitch constraints on neutron star structure}

Basing their work on the two-component model of pulsar glitches, Link et al. [269] derived a constraint on the ratio $I^{\mathrm{f}} / I$ of the moment of inertia $I^{\mathrm{f}}$ of the free superfluid neutrons in the crust to the total moment of inertia $I$ of the Vela pulsar, from which they inferred an inequality involving the mass and radius of the pulsar. However, they neglected entrainment effects (see Sections 8.3.6 and 8.3.7), which can be very strong in the crust, as shown by Chamel [90, 91]. We will demonstrate here how the constraint is changed by including these effects, following the analysis of Chamel \& Carter [94].

The total angular momentum $J$ of a rotating neutron star is the sum of the angular momentum $J^{\mathrm{f}}$ of free superfluid neutrons in the crust and of the angular momentum $J^{\mathrm{c}}$ of the "crust" (this includes not only the solid crust but also the liquid core, as discussed in Section 12.4.2). As reviewed in Sections 10.2 and 10.3, momentum and velocity of each component are not aligned due to (nondissipative) entrainment effects. Likewise, it can be shown that the angular momentum of each component is a superposition of both angular velocities $\Omega_{\mathrm{f}}$ and $\Omega_{\mathrm{c}}$ [94];

$$
\begin{aligned}
& J^{\mathrm{f}}=I^{\mathrm{ff}} \Omega_{\mathrm{f}}+I^{\mathrm{fc}} \Omega_{\mathrm{c}}, \\
& J^{\mathrm{c}}=I^{\mathrm{cf}} \Omega_{\mathrm{f}}+I^{\mathrm{cc}} \Omega_{\mathrm{c}},
\end{aligned}
$$

where $I^{\mathrm{ff}}, I^{\mathrm{fc}}=I^{\mathrm{cf}}$ and $I^{\mathrm{cc}}$ are partial moments of inertia, which determine $I^{\mathrm{f}}=I^{\mathrm{ff}}+I^{\mathrm{fc}}$ and $I^{\mathrm{c}}=I^{\mathrm{cf}}+I^{\mathrm{cc}}$. As discussed in [94], $I^{\mathrm{fc}}=I^{\mathrm{cf}}$ is expected to be positive in the core and negative in the crust.

Let us denote (discontinuous) variations of some quantity $Q$ during a glitch by $\Delta Q$ and (continuous) variations of this quantity during the interglitch period by $\delta Q$, as illustrated in Figure 76 . The total angular momentum $J=J^{\mathrm{f}}+J^{\mathrm{c}}$ can be assumed to be conserved during a glitch, therefore,

$$
\Delta J^{\mathrm{f}}=-\Delta J^{\mathrm{c}}
$$

If no torque were acting on the neutron superfluid in the interglitch period, its angular momentum $J^{\mathrm{f}}$ would be conserved and we would have $\delta J^{\mathrm{f}}=0$. However, neutron superfluid is weakly coupled to the magnetically-braked crust via friction forces induced by the dissipative motion of quantized vortex lines, as discussed in Section 8.3.5. Consequently, $J^{\mathrm{f}}$ does not remain exactly constant but decreases $\delta J^{\mathrm{f}} \leq 0$,

$$
\delta \Omega_{\mathrm{f}} \leq-\frac{I^{\mathrm{fc}}}{I^{\mathrm{ff}}} \delta \Omega_{\mathrm{c}}
$$




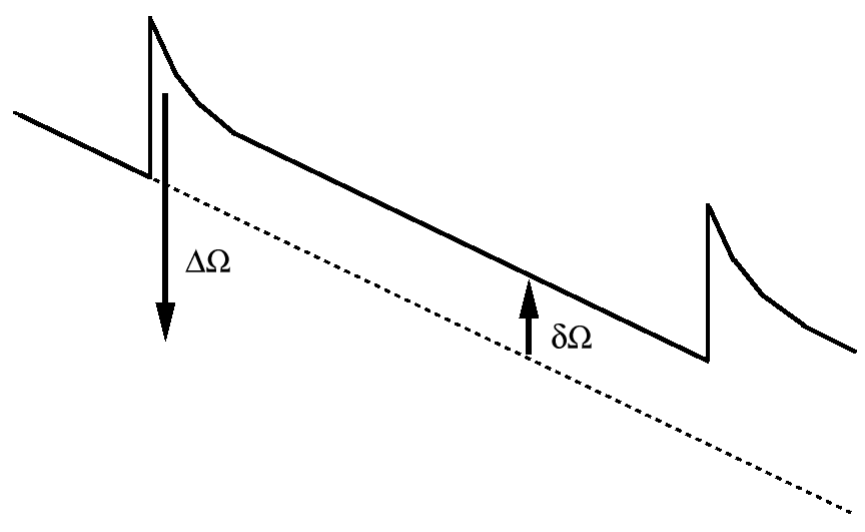

Figure 76: Schematic picture showing the variations $\Delta \Omega$ and $\delta \Omega$ of the pulsar angular frequency $\Omega$, during a glitch and in the interglitch period, respectively.

Friction effects prevent a long term build up of too large a deviation of the superfluid angular velocity $\Omega_{\mathrm{f}}$ from the externally observable value $\Omega=\Omega_{\mathrm{c}}$. This means that the average over many glitches (denoted by $\langle\ldots\rangle$ ) of the change of relative angular velocity $\Omega_{\mathrm{f}}-\Omega$ should be approximately zero

$$
\left\langle\Delta \Omega_{\mathrm{f}}+\delta \Omega_{\mathrm{f}}\right\rangle \simeq\langle\Delta \Omega+\delta \Omega\rangle .
$$

Combining Equations (283), (284) and (285), it can be shown that the partial moments of inertia are constrained by the following relation obtained by Chamel \& Carter [94]

$$
\frac{\left(I^{\mathrm{f}}\right)^{2}}{I I^{\mathrm{ff}}} \geq \mathcal{G} .
$$

The dimensionless coupling parameter $\mathcal{G}$ [269] depends only on observable quantities and is defined by

$$
\mathcal{G}=A_{g} \frac{\Omega}{\left|\dot{\Omega}_{\mathrm{av}}\right|},
$$

where $A_{g}$ is the glitch activity [286]

$$
A_{g}=\frac{1}{t} \sum_{i} \frac{\Delta \Omega_{i}}{\Omega}
$$

where the sum is over the glitches occurring during a time $t$ and $\dot{\Omega}_{\mathrm{av}}$ is the average spin-down rate. According to the statistical analysis of 32 glitches in 15 pulsars by Lyne et al. [276], the parameter $\mathcal{G}$ in Vela-like pulsars is $\mathcal{G} \simeq 0.017$. The coupling parameters for selected pulsars are shown in Figure 77. In particular, an analysis of the Vela pulsar shows that $\mathcal{G} \simeq 0.014$.

Microscopic calculations [90, 91] show that the ratio $I^{\mathrm{f}} / I^{\mathrm{ff}}$ is smaller than unity [94] (assuming that only neutron superfluid in the crust participates in the glitch phenomenon). We, thus, have

$$
\frac{I^{\mathrm{f}}}{I} \geq \frac{\left(I^{\mathrm{f}}\right)^{2}}{I I^{\mathrm{ff}}} \geq 0
$$

Adopting this upper bound and substituting in Equation (286), Link et al.[269] derived the following constraint on the mass $M$ and radius $R$ of the Vela pulsar

$$
R \geq 3.6+3.9 \frac{M}{M_{\odot}} \mathrm{km} .
$$




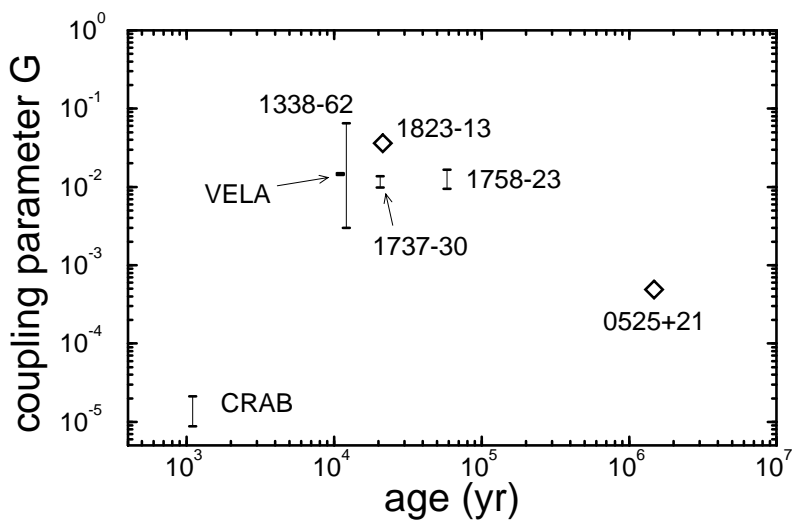

Figure 77: Coupling parameter $\mathcal{G}$ for selected glitching pulsars. From [269].

Let us emphasize that this constraint has been obtained by neglecting entrainment effects between neutron superfluid and crust. However, these effects are known to be very strong [90], so that the inequality (290) should be taken with a grain of salt.

\subsection{Gravitational wave asteroseismology}

The development of gravitational wave detectors like LIGO [67], VIRGO [208], TAMA300 [301] and GEO600 [299] is opening up a new window of astronomical observations. With a central density on the order of $\sim 10^{15} \mathrm{~g} \mathrm{~cm}^{-3}$, neutron stars are among the most compact objects in the universe, and could be efficient sources of gravitational waves. The existence of such waves predicted by Einstein's theory of general relativity was beautifully confirmed by the observations of the binary pulsar PSR $1913+16$ by Russel Hulse and Joseph Taylor (who were awarded the Nobel Prize in 1993 [306]). To compare the importance of general relativistic effects in binary pulsars with those around ordinary stars like the Sun, let us remember that the pulsar's periastron in PSR 1913+16 advances every day by the same amount as Mercury's perihelion advances in a century!

\subsubsection{Mountains on neutron stars}

A spherical neutron star does not radiate gravitational waves, in accordance with Birkhoff's theorem. This is still true for an axially-symmetric neutron star. However, a neutron star with nonaxial deformations, rigidly rotating with the angular frequency $\Omega$, radiates gravitational waves and thus loses energy at a rate given by the formula

$$
\dot{E}_{\mathrm{GR}}=-\frac{32}{5} \frac{G}{c^{5}} I^{2} \epsilon^{2} \Omega^{6}
$$

where $\epsilon$ is a dimensionless parameter characterizing deformations of the star. Pulsar timing data can be used to derive an upper bound on this parameter $\epsilon$. Since the energy radiated away in gravitational waves can be at most equal to the loss of kinetic energy due to the spinning down of the pulsar, this implies

$$
\epsilon<3 \times 10^{-9}\left(\frac{P}{1 \mathrm{~ms}}\right)^{3 / 2}\left(\frac{\dot{P}}{10^{-19}}\right)^{1 / 2}\left(\frac{I}{10^{45} \mathrm{~g} \mathrm{~cm}^{2}}\right)^{-1 / 2}
$$

where $P, \dot{P}$ and $I$ are, respectively, the pulsar's period, period derivative and moment of inertia. 
The parameter $\epsilon$ can be constrained, independent of the pulsar timing data, by direct observations with gravitational wave detectors [220]

$$
\epsilon<0.237 \frac{h}{10^{-24}} \frac{d}{1 \mathrm{kpc}}\left(\frac{f}{1 \mathrm{~Hz}}\right)^{-2}\left(\frac{I}{10^{45} \mathrm{~g} \mathrm{~cm}^{2}}\right)^{-1},
$$

where $h$ is the amplitude of a gravitational wave signal, $f$ the neutron star's spin frequency and $d$ is the distance to the source. 78 radio pulsars have recently been observed using the LIGO detector [1]. The analysis of the data imposes upper limits on the maximum amplitude $h$ of the gravitational waves emitted by these pulsars. The two inequalities (292) and (293) can be combined to put constraints on the moment of inertia $I$ and on the parameter $\epsilon$ of a given pulsar. The example of the Crab pulsar [2] is shown in Figure 78. Note that the constraint from the gravitational wave data has already reached the level of the spin-down limit.

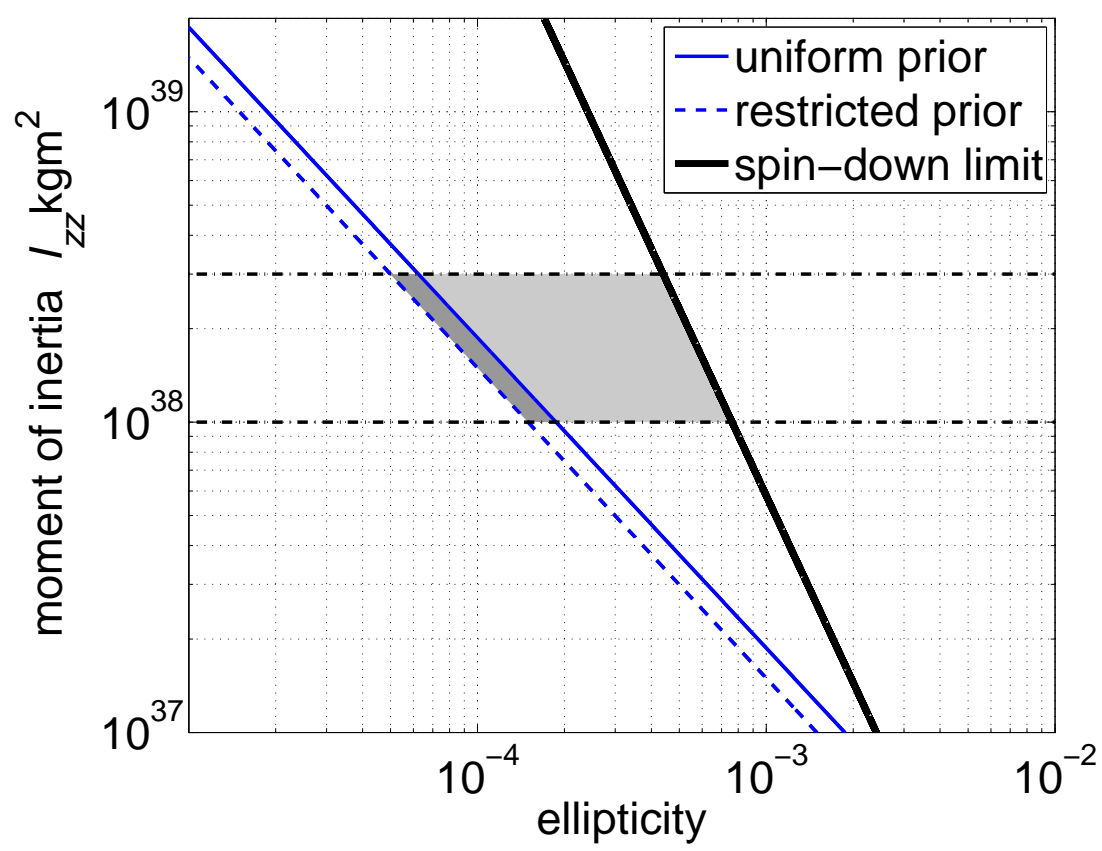

Figure 78: The moment of inertia $I$ vs. the deformation parameter $\epsilon$ for the Crab pulsar over the S5 run of the LIGO detector. The areas to the right of the diagonal lines are the experimentally excluded regions. The horizontal lines represent theoretical upper and lower limits on the moment of inertia. The lines labelled "uniform prior" and "restricted prior" are the results obtained respectively without and with prior information on the gravitational wave signal parameters. From [2].

The crust of a neutron star contains only a very small percentage of the mass of the star. Nevertheless, its elastic response to centrifugal, magnetic and tidal forces determines the overall shape of the star. The presence of mountains on the surface of the star leads to a nonvanishing value of the parameter $\epsilon$. If the star is rotating around one of the principal axes of the inertia tensor, $\epsilon$ is given by [373]

$$
\epsilon=\frac{I_{1}-I_{2}}{I}
$$

where $I_{1}$ and $I_{2}$ are the moments of inertia of the star with respect to the principal axes orthogonal to the rotation axis. The topography of the surface of a neutron star depends on the elastic 
properties of the solid crust. In particular, the size of the highest mountain depends on the breaking strain $\sigma_{\max }$, beyond which the crust will crack. This parameter is believed to lie in the range $10^{-5}-10^{-2}$, as argued by Smoluchowski [382]. Considering small perturbations in a Newtonian star composed of an incompressible liquid of density $\rho$, surrounded by a thin crust with a constant shear modulus $\mu$, the deformation parameter $\epsilon$ is given by the formula [192]

$$
\epsilon=\frac{9}{80} \frac{\mu \sigma_{\max }}{G \rho^{2}} \frac{\Delta R}{R^{3}},
$$

where $\Delta R$ the thickness of the solid crust. Adopting the value $\mu=10^{30} \mathrm{~g} \mathrm{~cm}^{-1} \mathrm{~s}^{-2}$ (see Section 7.1), $\epsilon$ can be written as

$$
\epsilon \simeq 5 \times 10^{-5}\left(\frac{\sigma_{\max }}{10^{-3}}\right)\left(\frac{1.4 M_{\odot}}{M}\right)^{2}\left(\frac{R}{10 \mathrm{~km}}\right)^{3}\left(\frac{\Delta R}{1 \mathrm{~km}}\right) .
$$

The size of the highest mountain of order $\sim \epsilon R$ can be roughly estimated from the inequality (292). For instance, the mountains on the surface of the Crab pulsar could be as high as a few meters, while in PSR 1957+20 they cannot exceed a few microns! A neutron star can also be deformed by its magnetic field (Section 6.4, see also [193] and references therein). The deformation depends on the configuration of the magnetic field and scales like

$$
\epsilon \propto \frac{B^{2} R^{4}}{G M^{2}}
$$

For a Newtonian star composed of an incompressible liquid, the deformation induced by an internal magnetic field is approximately given by [193]

$$
\epsilon \simeq 10^{-12}\left(\frac{R}{10 \mathrm{~km}}\right)^{4}\left(\frac{M}{1.4 M_{\odot}}\right)^{-2}\left(\frac{\widetilde{B}}{10^{12} \mathrm{G}}\right)^{2},
$$

where $\widetilde{B}$ is a suitably-averaged magnetic field. Comparing Equations (296) and (298) we conclude that for a canonical neutron star $\left(M=1.4 M_{\odot}, R=10 \mathrm{~km}, \Delta R=1 \mathrm{~km}, \widetilde{B}=10^{12} \mathrm{G}\right)$ the deformations induced by the magnetic field are much smaller than those supportable by the elasticity of the crust. However, for $\widetilde{B} \sim 10^{15} \mathrm{G}$, characteristic of magnetars, both deformations have comparable magnitudes. Moreover, it has been shown that neutron stars with large toroidal fields evolve into configurations, where the angular momentum of the star is orthogonal to the magnetic axis. Such configurations are associated with strong gravitational wave emission (see [105] and references therein).

\subsubsection{Oscillations and precession}

The presence of magnetic fields inside neutron stars or mountains on the surface are not the only mechanisms for the emission of gravitational waves. Time-dependent nonaxisymmetric deformations can also be caused by oscillations. For instance, a neutron star with a solid crust, rotating about some axis (i.e. not aligned with any principal axis of the stellar moment of inertia tensor) will precess. For a small wobble angle $\theta$, the deformation parameter is given by [373]

$$
\epsilon=\frac{\theta}{4} \frac{I_{1}-I_{3}}{I}
$$

where $I_{1}, I_{2}$ and $I_{3}$ are the principal moments of inertia (assuming $I_{1}=I_{2}$ ), $\theta$ is the angle between the direction of the angular momentum and the stellar symmetry axis. Observational evidence 
of long-period precessions have been reported in PSR 1828-11 [387], PSR B1642-03 [371] and RXJ 0720.4-3125 [180].

A large number of different nonaxisymmetric neutron-star-oscillation modes exists, for instance, in the liquid surface layers ("ocean"), in the solid crust, in the liquid core and at the interfaces between the different regions of the star. These oscillations can be excited by thermonuclear explosions induced by the accretion of matter from a companion star, by starquakes, by dynamic instabilities growing on a timescale on the order of the oscillation period or by secular instabilities driven by dissipative processes and growing on a much longer timescale. Oscillation modes are also expected to be excited during the formation of the neutron star in a supernova explosion. The nature of these modes, their frequency, their growing and damping timescales depend on the structure and composition of the star (for a review, see, for instance, [285, 244, 12]).

\subsubsection{Crust-core boundary and r-mode instability}

Of particular astrophysical interest are the inertial modes or Rossby waves (simply referred to as r-modes) in neutron star cores. They can be made unstable by the radiation of gravitational waves on short timescales of a few seconds in the most rapidly-rotating neutron stars [17]. However, the growth of these modes can be damped. One of the main damping mechanisms is the formation of a viscous boundary Ekman layer at the crust-core interface [49] (see also [164] and references therein). It has been argued that the heat dissipated in this way could even melt the crust [265]. The damping rate depends crucially on the structure of bottom layers of the crust and scales like $(\delta v / v)^{2}$, where $\delta v$ is the slippage velocity at the crust-core interface [263]. Let us suppose that the liquid in the core does not penetrate inside the crust, like a liquid inside a bucket. The slippage velocity in this case is very large $\delta v \sim v$ and as a consequence the r-modes are strongly damped. However, these assumptions are not realistic. First of all, the crust is not perfectly rigid, as discussed in Section 7. On the contrary, the crust is quite "soft" to shear deformations because $\mu / P \sim 10^{-2}$, where $\mu$ is the shear modulus and $P$ the pressure. The oscillation modes of the liquid core are coupled to the elastic modes in the crust, which results in much smaller damping rates $[263,163]$. Besides, the transition between the crust and the core might be quite smooth. Indeed, neutron superfluid in the core permeates the inner crust and the denser layers of the crust could be formed of nuclear "pastas" with elastic properties similar to those of liquid crystals (see Section 7.2). The slippage velocity at the bottom of the crust could, therefore, be very small $\delta v \ll v$. Consequently, the Ekman damping rate of the r-modes could be much weaker than the available estimates. If the crust were purely fluid, the damping rate would be vanishingly small. However the presence of the magnetic field would also affect the damping time scale and should be taken into account [289,238]. Besides the character of the core oscillation modes is likely to be affected by coupling with the crust. The role of the crust in the dissipation of the r-mode instability is, thus, far from being fully understood. Finally, let us mention that by far the strongest damping mechanism of r-modes, due to a huge bulk viscosity, may be located in the inner neutron star core, provided it contains hyperons (see, e.g., [182, 264] and references therein).

\subsection{Giant flares from Soft Gamma Repeaters}

The discovery of quasi-periodic oscillations (QPO) in X-ray flux following giant flares from Soft Gamma Repeaters (SGR) has recently triggered a burst of intense theoretical research. Oscillations were detected at frequencies 18,26, 29, 92.5, 150, 626.5 and $1837 \mathrm{~Hz}$ during the spectacular December 27, 2004 giant flare (the most intense ever observed in our Galaxy) from SGR 1806-20 [214, 421, 397] and at 28, 54, 84 and $155 \mathrm{~Hz}$ during the August 27, 1998 giant flare from SGR 1900+14 [396]. Evidence has also been reported for oscillations at $43.5 \mathrm{~Hz}$ during the March 5, 1979 event in SGR 0526-66 [34]. Soft Gamma Repeaters (SGR) are believed 
to be strongly-magnetized neutron stars or magnetars endowed with magnetic fields as high as $10^{14}-10^{15} \mathrm{G}$ (for a recent review, see, for instance, [426] and references therein; also see the home page of Robert C. Duncan [128]). Giant flares are interpreted as crustquakes induced by magnetic stresses. Such catastrophic events are likely to be accompanied by global seismic vibrations, as observed by terrestrial seismologists after large earthquakes. Among the large variety of possible oscillation modes, torsional shear modes in the crust are the most likely [129]. Shear flow in the crust is illustrated in Figure 58. If confirmed, this would be the first direct detection of oscillations in a neutron star crust.

Neglecting the effects of rotation and magnetic fields, and ignoring the presence of neutron superfluid in the inner crust, but taking into account the elasticity of the crust in general relativity, the frequency of the fundamental toroidal crustal mode of multipolarity $\ell=2$ (the case $\ell=1$ corresponding to the crust uniformly rotating around the static core is ignored), is approximately given by [359]

$$
f_{n=0, \ell=2} \simeq 2 \pi v_{t} \sqrt{\frac{1-r_{\mathrm{g}} / R}{R r_{\mathrm{cc}}}}, \quad f_{n=0, \ell} \simeq f_{n=0, \ell=2} \sqrt{(\ell-1)(\ell+2)},
$$

where $r_{\mathrm{g}}$ is the Schwarzschild radius of the star, $r_{\mathrm{cc}}$ the radius of the crust and $v_{t}$ is the speed of shear waves propagating in an angular direction and polarized in the mutually orthogonal angular direction. The frequencies of the higher fundamental $(n=0)$ modes scale like

$$
f_{n=0, \ell} \simeq f_{n=0, \ell=2} \sqrt{(\ell-1)(\ell+2)} .
$$

The frequencies of overtones $n>0$ are independent of $\ell$ to a good approximation (provided $\ell$ is not too large compared to $n$ ), and can be roughly estimated as

$$
f_{n>0} \simeq\left(1-\frac{r_{\mathrm{g}}}{R}\right) \frac{2 \pi^{2} n v_{r}}{\Delta R},
$$

where $v_{r}$ is the speed of shear waves propagating radially with polarization in an angular direction. For a reasonable crustal equation of state, the crust thickness $\Delta R \equiv R-r_{\text {cc }}$ can be estimated as [359]

$$
\frac{\Delta R}{R} \simeq\left[1+21.5 \frac{r_{\mathrm{g}}}{R}\left(1-\frac{r_{\mathrm{g}}}{R}\right)\right]^{-1}
$$

If the crust is isotropic, the velocities $v_{r}$ and $v_{t}$ are equal.

The analysis of QPOs in SGRs can potentially provide valuable information on the properties of the crust and, more generally, on the structure of neutron stars. The identification of both the $29 \mathrm{~Hz}$ and $626.5 \mathrm{~Hz}$ QPOs in the 2004 giant flare from SGR 1806-20 as the fundamental $n=0$, $\ell=2$ toroidal mode and the first overtone $n=1, \ell=1$, respectively, puts stringent constraints on the mass and radius of the star, as shown in Figure 79. This constraint rules out some stiff equations of state based on the relativistic mean field theory proposed by Glendenning [166]. The $28 \mathrm{~Hz}$ and $54 \mathrm{~Hz}$ QPOs in the 1998 flare from SGR 1900+14 have been identified with the $\ell=2$ and $\ell=4$ toroidal modes, respectively. In this case, the mass and radius are much less constrained, as can be seen in Figure 80. The identification of higher frequency QPOs is more controversial.

The effect of rotation on oscillation modes is to split the frequency of each mode with a given $\ell$ into $2 \ell+1$ frequencies. It has recently been pointed out that some of the resulting modes might, thus, become secularly unstable, according to the Chandrasekhar-Friedman-Schutz (CFS) criterion [411]. The study of oscillation modes becomes even more difficult in the presence of a magnetic field. Roughly speaking, the effects of the magnetic field increase the mode frequencies [129, 290, 328, 257, 385]. Simple Newtonian estimates lead to an increase of the frequencies by a factor $\sqrt{1+\left(B / B_{\mu}\right)^{2}}$, where $B_{\mu} \equiv \sqrt{4 \pi \mu}$ is expressed in terms of the shear modulus $\mu$ [129]. 


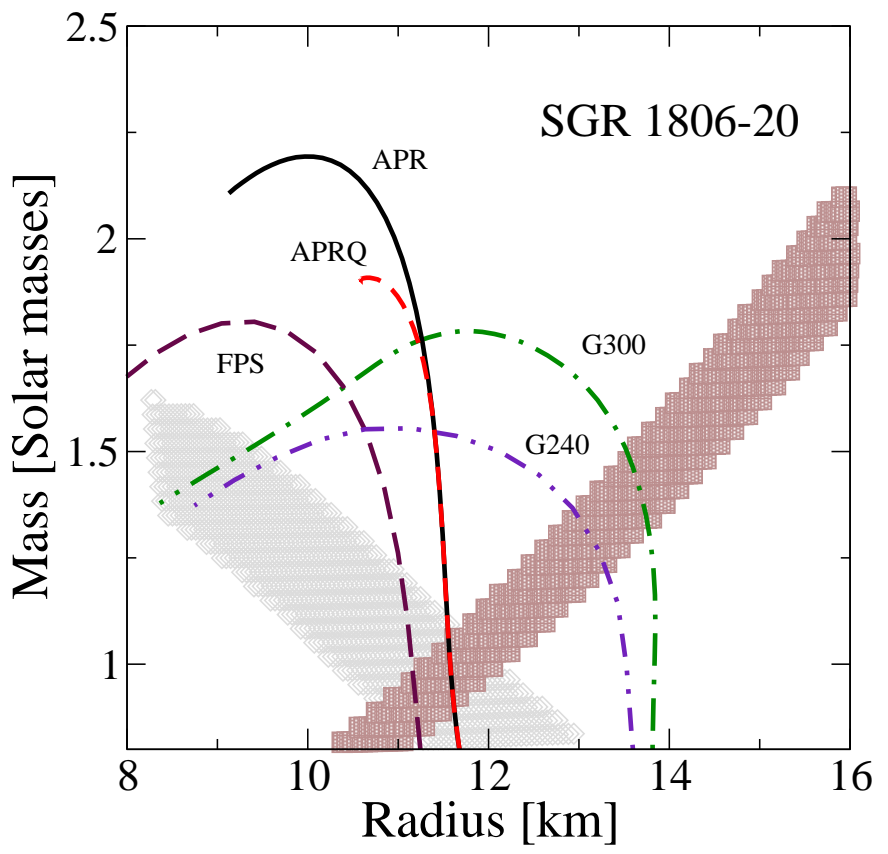

Figure 79: Constraints on the mass and radius of SGR 1806-20 obtained from the seismic analysis of quasi-periodic oscillations in X-ray emission during the December 27, 2004 giant flare. For comparison, the mass-radius relation for several equations of state is shown (see [359] for further details).

Sotani and et al. [385] recently carried out calculations in general relativity with a dipole magnetic field and found numerically that the frequencies are increased by a factor $\sqrt{1+\alpha_{n, \ell}\left(B / B_{\mu}\right)^{2}}$, where $\alpha_{n, \ell}$ is a numerical coefficient. However, it has been emphasized by Messios et al. [290] that the effects of the magnetic field strongly depend on its configuration. The most important effect is to couple the crust to the core so that the whole stellar interior vibrates during a giant flare [165]. Low frequency QPOs could, thus, be associated with magnetohydrodynamic (MHD) modes in the core [262].

Another important aspect to be addressed is the presence of neutron superfluid, which permeates the inner crust. The formalism for treating a superfluid in a magneto-elastic medium has been recently developed both in general relativity [85] and in the Newtonian limit [73, 72], based on a variational principle. This formalism has not yet been applied to study oscillation modes in magnetars. However, we can anticipate the effects of the neutron superfluid using the two-fluid description of the crust reviewed in Section 10.2. Following the same arguments as for two-fluid models of neutron star cores [13], two classes of oscillations can be expected to exist in the inner crust, depending on whether neutron superfluid is co-moving or countermoving with the crust. The countermoving modes are predicted to be very sensitive to entrainment effects, which are very strong in the crust [90, 91].

The neutron-star-oscillation problem deserves further theoretical study. The prospect of probing neutron star crusts by analyzing the X-ray emission of giant magnetar flares is very promising.

\subsection{Low mass X-ray binaries}

As reviewed in Section 4, the accretion of matter (mainly hydrogen and helium) onto the surface of neutron stars triggers thermonuclear fusion reactions. Under certain circumstances, these reactions can become explosive, giving rise to X-ray bursts. The unstable burning of helium ash produced 


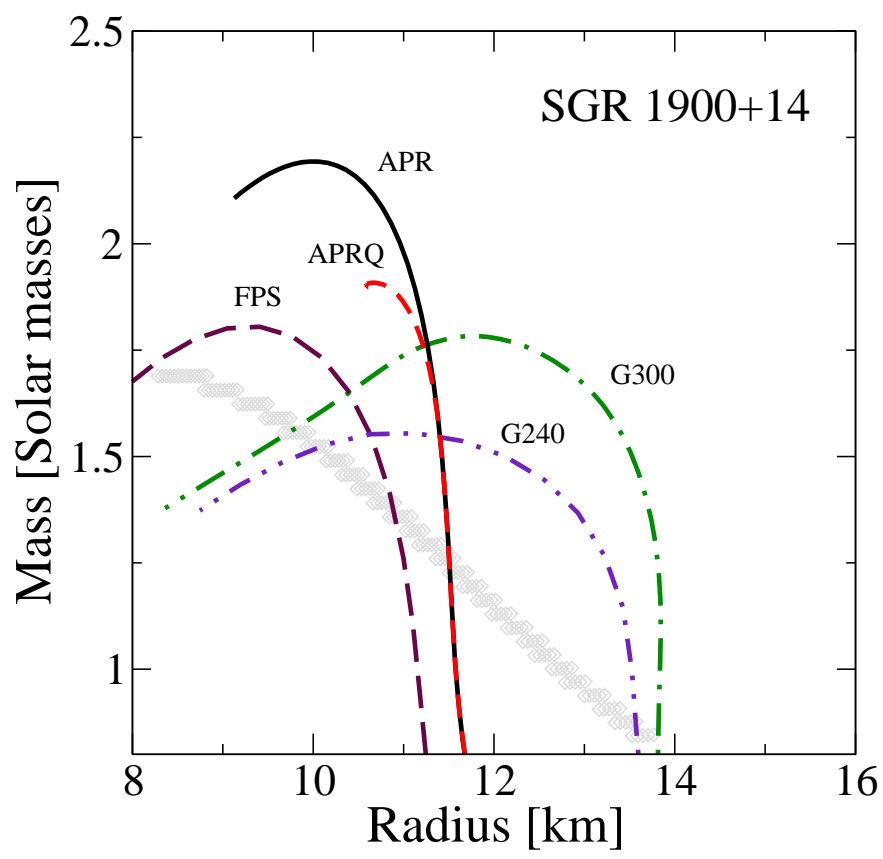

Figure 80: Constraints on the mass and radius of SGR 1900+14 obtained from the seismic analysis of the quasi-periodic oscillations in the X-ray emission during the August 27, 1998 giant flare. For comparison, the mass-radius relation for several equations of state is shown (see [359] for further details). From [359].

by the fusion of accreted hydrogen, is thought to be at the origin of type I X-ray bursts. A new type of X-ray burst has been recently discovered. These superbursts are a thousand times more energetic than normal bursts and last several hours compared to a few tens of seconds, but occur much more rarely. These superbursts could be due to the unstable burning of ${ }^{12} \mathrm{C}$ accumulated from He burning. The mass of ${ }^{12} \mathrm{C}$ fuel has to be as high as $10^{-9} M_{\odot}$ to get $E_{\text {burst }} \sim 10^{42} \mathrm{erg}$. It can be seen from Figure 39 that ${ }^{12} \mathrm{C}$ ignition has to occur at $\rho \sim 10^{9} \mathrm{~g} \mathrm{~cm}^{-3}$ at a depth of $\sim 30 \mathrm{~m}$. At the accretion rates characteristic of typical superbursters $\left(\dot{M} \sim(1-3) \times 10^{-9} M_{\odot} / \mathrm{y}\right)$, this would correspond to recurrence times of a few years. It also seems that crustal heating might be quite important to getting such a relatively low ignition density of ${ }^{12} \mathrm{C}$ [178]. The ignition conditions are very sensitive to the thermal properties of the crust and core [104]. X-ray observations of low-mass X-ray binaries thus provide another way of probing the interior of neutron stars, both during thermonuclear bursting episodes and during periods of quiescence as discussed below.

\subsubsection{Burst oscillations}

During the past ten years, millisecond oscillations have been discovered in X-ray bursts in low mass X-ray binaries as illustrated in Figure 81 (see, for instance, [392] and references therein for a recent review). Such oscillations have been observed during the rise time of bursts, as well as at later times in the decay phase. The observations of burst oscillations in the accreting millisecond pulsars SAX J1808.4-3658 [213, 279, 89] and XTE J1814-338 [395] firmly established that burst oscillations occur close to the spin frequency of the neutron star. This conclusion was further supported by the discovery of about 500,000 highly coherent pulsation cycles at $582 \mathrm{~Hz}$ during a superburst from the low mass X-ray binary 4U 1636-356 [394]. This suggests that burst oscillations arise from some nonuniformities on the neutron star surface. In the burst rise, the oscillations are likely to be caused by the presence of hot spots induced by the ignition of nuclear 
burning. This interpretation naturally explains why oscillation amplitudes decrease with time as the burning region spreads over the entire surface [398], as shown in Figure 82. Nevertheless, this model cannot explain the oscillations detected in the burst tail, since the duration of the burst (of the order 10-30 seconds) is much larger than the spreading time of thermonuclear burning (typically less than 1 second). Surface inhomogeneities during the cooling phase could be produced by the dynamic formation of vortices driven by the Coriolis force [386] and by nonradial surface oscillations [196]. The outer envelope of an accreting neutron star is formed of three distinct regions: a hot bursting shell, an ocean and the solid outer crust, so that many different oscillation modes could be excited during a burst. The observed frequencies and positive frequency drifts are consistent with shallow surface waves excited in the hot bursting layer changing into crustal interface waves in the ocean as the surface cools [330]. This model can also explain the energy dependence of the burst oscillation amplitude [331]. The interface waves resemble shallow surface waves, but with a large radial displacement at the ocean/crust boundary due to the elasticity of the crust [329]. The frequency of the interface wave is reduced by a factor $\sqrt{\mu / P} \sim 0.1$, where $\mu$ is the shear modulus and $P$ the pressure, as compared to a rigid surface. The frequencies of these modes depend on the composition of the neutron star surface layers. This raises the exciting possibility of probing accreting neutron star crusts with X-ray burst oscillations. Very recently evidence has come forth for burst oscillations at a frequency of $1122 \mathrm{~Hz}$ in the X-ray transient XTE J1739-285 [227]. If confirmed, it would imply that this system contains the fastest spinning neutron star ever discovered. Since the spinning rate is limited by the mass shedding limit, these observations would thus put constraints on the gravitational mass $M$ and circumferential equatorial radius $R$ of the neutron star in XTE J1739-285 [45]

$$
R<15.52\left(\frac{M}{1.4 M_{\odot}}\right)^{1 / 3} \mathrm{~km} .
$$

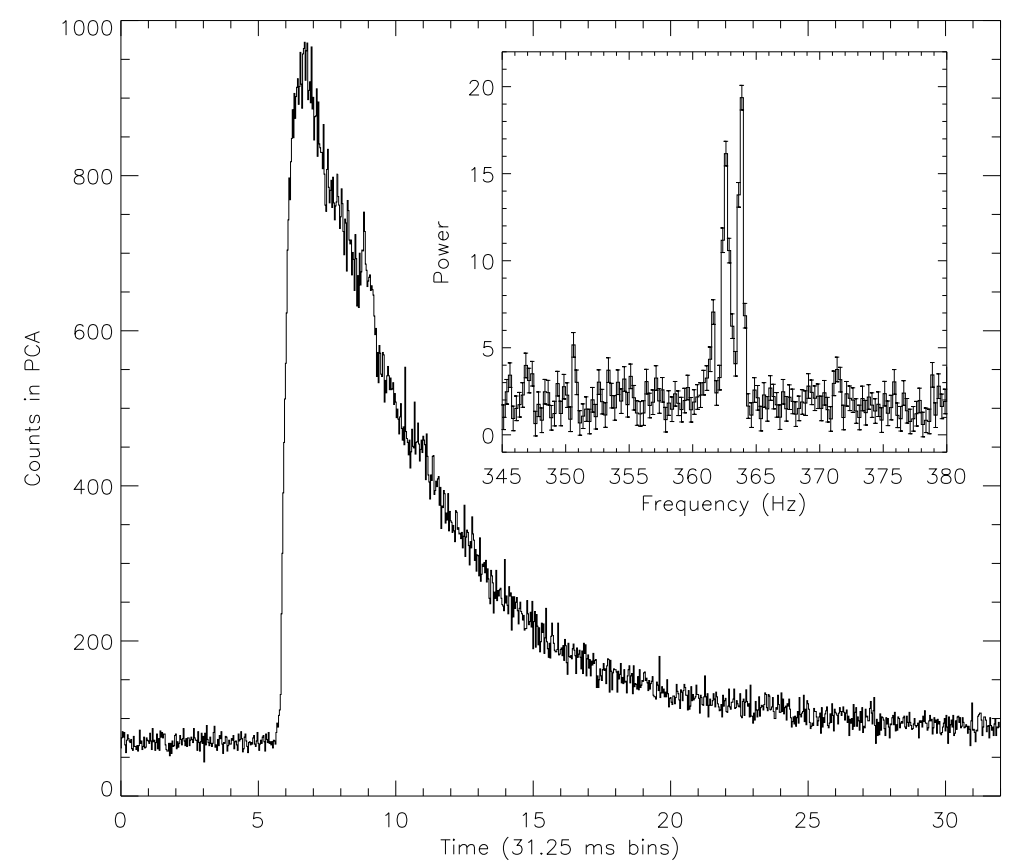

Figure 81: Oscillations detected in an X-ray burst from 4U $1728-34$ at frequency $\sim 363 \mathrm{~Hz}$ [399]. 

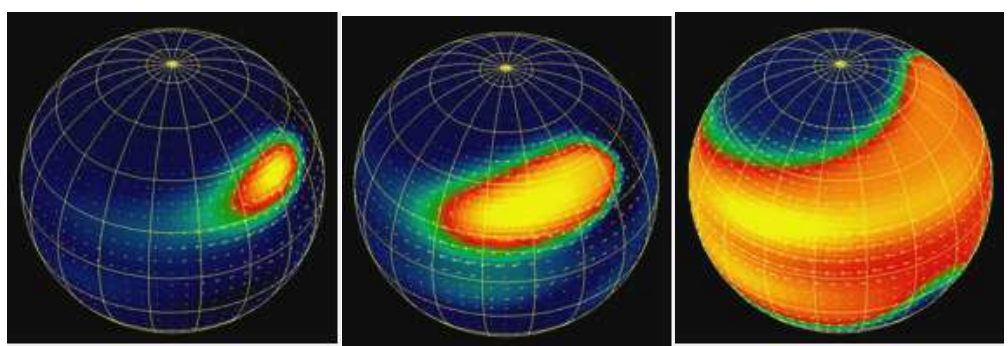

Figure 82: Spreading of a thermonuclear burning hotspot on the surface of a rotating neutron star simulated by Spitkovsky [386] (from http://www.astro.princeton.edu/ anatoly/).

\subsubsection{Soft X-ray transients in quiescence}

The phenomenon of deep crustal heating appears to be relevant for the understanding of the thermal radiation observed in soft X-ray transients (SXTs) in quiescence, when the accretion from a disk is switched off or strongly suppressed. Typically, the quiescent emission is much higher than it would be in an old cooling neutron star. It has been suggested that this is because the interiors of neutron stars in SXTs are heated up during relatively short periods of accretion and bursting by the nonequilibrium processes associated with nuclear reactions taking place in the deep layers of the crust ([60], see also Section 4.3). The deep crustal heating model, combined with appropriate models of the neutron star atmosphere and interior, is used to explain measured luminosities of SXTs in quiescence. The luminosity in quiescence depends on the structure of neutron star cores, and particularly on the rate of neutrino cooling. This opens up the new possibility of exploring the internal structure and equation of state of neutron stars (see [102, 358, 429, 430] and references therein).

Let us denote the duration of the accretion stage, with accretion rate $\dot{M}_{\mathrm{a}}$, by $t_{\mathrm{a}}$, and the duration of quiescence between two active periods by $t_{\mathrm{q}}$, with $t_{\mathrm{q}} \gg t_{\mathrm{a}}$. After a few thousands of accretion-quiescence cycles, an SXT reaches a steady thermal state with the well-defined thermal structure of quiescence. This thermal structure is fully determined by the time-averaged accretion rate $\langle\dot{M}\rangle=t_{\mathrm{a}} \dot{M}_{\mathrm{a}} /\left(t_{\mathrm{a}}+t_{\mathrm{q}}\right)$. A steady state in quiescence satisfies the global energy balance "on average". The heat associated with nuclear $\mathrm{H}$ and He burning and X-ray bursting during $t_{\mathrm{a}}$ is nearly completely radiated away, and therefore does not contribute to the steady-state energy balance. Therefore, to a good approximation, the sum of the total average cooling rates (photon surface and neutrino volume emission) is balanced by deep crustal heating during an accretion period. Except for a thin blanketing envelope, the interior of an SXT in quiescence is isothermal, with temperature $T_{\text {int }}$. A blanketing envelope separates the isothermal interior from the surface, where the photons are emitted with a spectrum formed in a photosphere of effective temperature $T_{\text {eff }}$. Therefore,

$$
L_{\gamma}\left(T_{\text {eff }}\right)+L_{\nu}\left(T_{\text {int }}\right)=L_{\mathrm{dh}}(\langle\dot{M}\rangle),
$$

where the total time-averaged deep-crustal heating rate is

$$
L_{\mathrm{dh}}(\langle\dot{M}\rangle)=Q_{\mathrm{tot}}\langle\dot{M}\rangle / m_{u} \approx 6.03 \times 10^{33}\left\langle\dot{M}_{-10}\right\rangle \frac{Q_{\mathrm{tot}}}{\mathrm{MeV}} \mathrm{erg} \mathrm{s}^{-1},
$$

and $Q_{\mathrm{tot}}$ is the total heat released per accreted nucleon.

As can be seen in Figure $83, Q_{\text {tot }}=1.5 \mathrm{MeV} /$ nucleon is consistent with SXTs observations. However, different sources require different neutron star masses $M$. This is because the core neutrino cooling rate depends on the mass of the inner core, where the direct Urca process is possible. As shown in Figure 84, $Q_{\text {tot }}=0.15 \mathrm{MeV} /$ nucleon (and a fortiori $Q_{\text {tot }}=0$ ) would contradict observations of Aql X-1, RX 1709-2639 and 4U 1608-52 in quiescence.

Living Reviews in Relativity

http://www. livingreviews.org/Irr-2008-10 


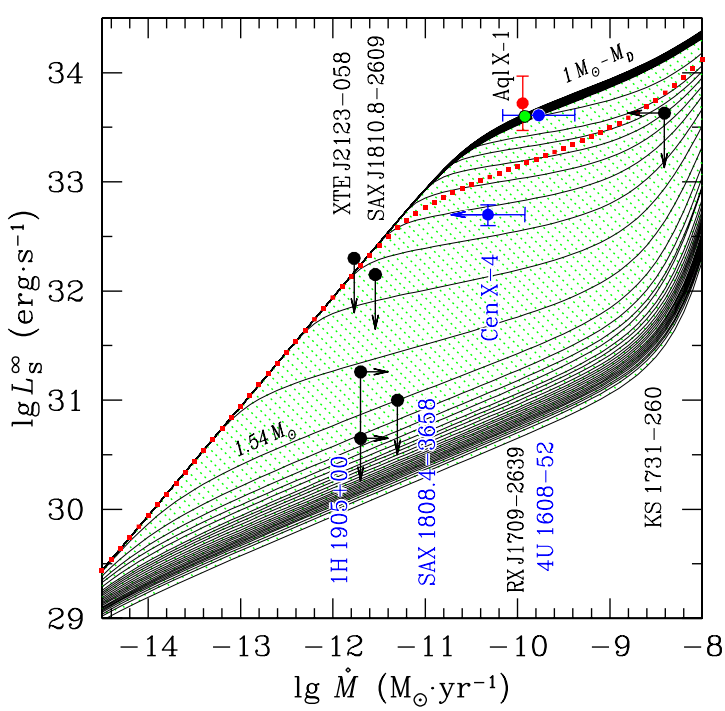

Figure 83: X-ray luminosities of SXTs in quiescence vs. time-averaged accretion rates. The heating curves correspond to different neutron star masses, increasing from the top to the bottom, with a step of $\Delta M=0.02 M_{\odot}$. The EoS of the core is moderately stiff, with $M_{\max }=1.977 M_{\odot}[340]$. The model of a strong proton and a weak neutron superfluidity is assumed [261]. The highest curve (hottest stars) corresponds to $M=M_{\odot}$, and the lowest one (coldest stars) to $M=M_{\max }$. The upper bundle of coalescing curves corresponds to masses $M_{\odot} \leq M \leq M_{\mathrm{D}}$, where a star of mass $M_{\mathrm{D}}$ has a central density equal to the threshold for the direct Urca process. The red dotted line represents thermal states of a "basic model" (nonsuperfluid core, slow cooling via a modified Urca process). Total deep heat released per accreted nucleon is $Q_{\text {tot }}=1.5 \mathrm{MeV}$. Figure made by K.P. Levenfish. For a further description of neutron star models and observational data see Levenfish \& Haensel [261].

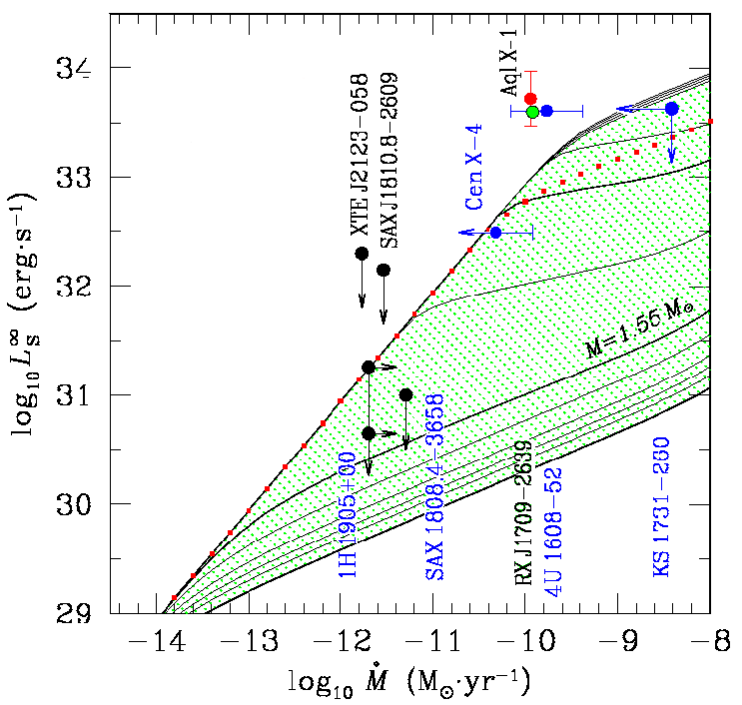

Figure 84: Same as Figure 83 but assuming ten times smaller $Q_{\text {tot }}=0.15 \mathrm{MeV}$. Such a low crustal heating is contradicted by the measured luminosities of three SXTs: Aql X-1, RXJ1709-2639, and 4U 1608-52. Figure made by K.P. Levenfish. 


\subsubsection{Initial cooling in quasi-persistent SXTs}

Quasi-persistent SXTs, with accretion periods lasting for years - decades, might be particularly useful for studying the structure of neutron star crusts. This is because one can observe their thermal relaxation between the accreting and quiescent stages. For standard SXTs, with accretion lasting days - weeks, such relaxation cannot be detected, because crustal heating due to accretion is too small. On the contrary, thermal relaxation toward the quiescent state for KS 1731-260 (after accreting over $12.5 \mathrm{y}$ ) and for MXB 1659-29 (after accreting over $2.5 \mathrm{y}$ ), called "initial cooling", was observed [66]. Let us consider the thermal relaxation of KS 1731-260. After 12.5 y of accretion and deep crustal heating, the crust and the surface became significantly hotter than in the quiescent state. The cooling curve depends on crust properties, such as thermal conductivity (Section 9), thickness (Section 6), distribution of heat sources (Section 4), and neutrino emissivity (Section 11). Some of these properties depend strongly on the crust structure, as illustrated in Figure 85. Modeling of the initial cooling curve can hopefully constrain the crust physics [358, $66,379]$. An example of such modeling is shown in Figure 86. The cooling curve is much more sensitive to the crust physics than to that of the dense core. For example, an amorphous crust, with its low thermal conductivity (see Figure 85), yields a too slow relaxation (see Figure 86 ). Moreover, the star has to be massive to have a sufficiently thin crust to relax sufficiently rapidly.

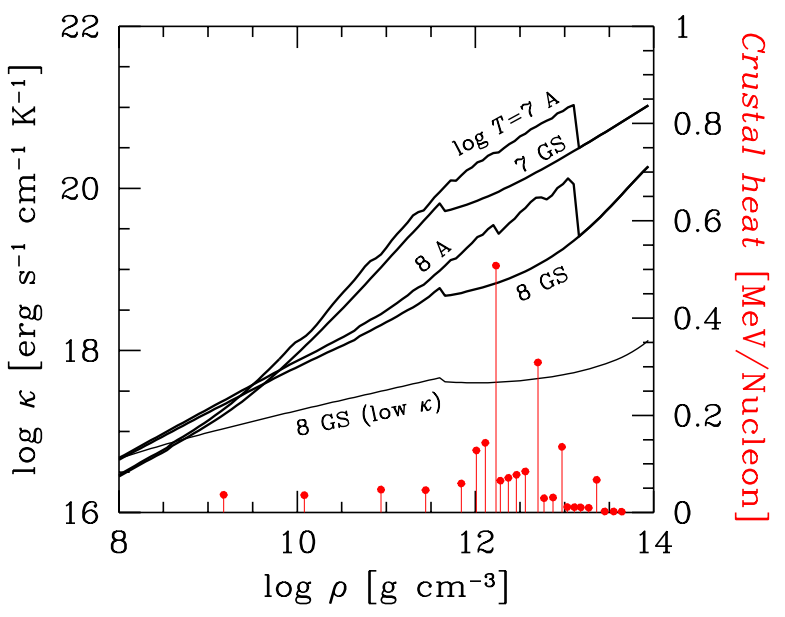

Figure 85: Left vertical axis: thermal conductivity of neutron star crust vs. density, at $\log _{10}(T)[K]=7$ and 8. GS - pure ground state crust. A - pure accreted crust. GS (low $\kappa$ ) - amorphous ground state crust. Right vertical axis - deep crustal heating per accreted nucleon in a thin heating shell, vs. density, according to $A_{\mathrm{i}}=56$ model of Haensel \& Zdunik [188]. From Shternin et al. [379]. Figure made by P.S. Shternin. 


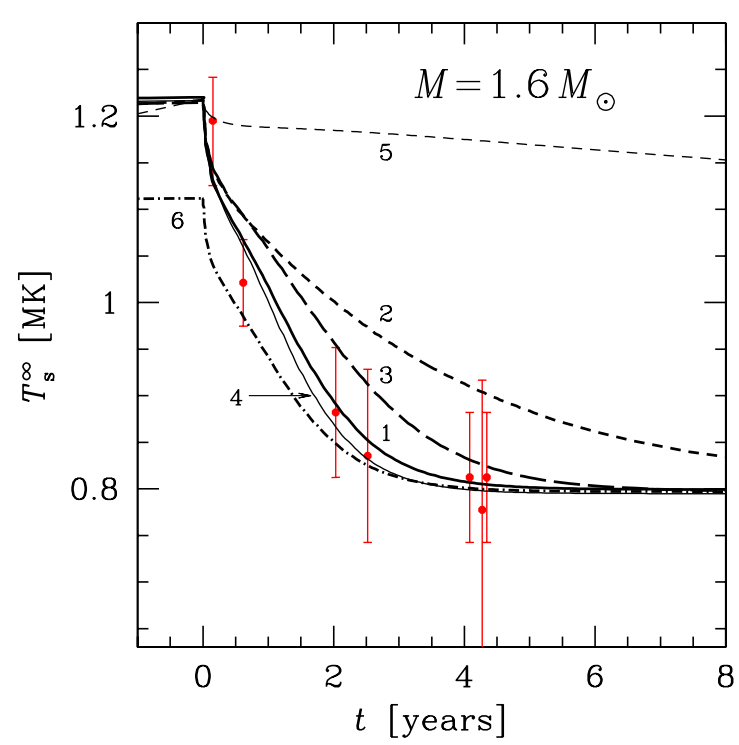

Figure 86: Theoretical cooling curves for KS 1731-260 relaxing toward a quiescent state; observations expressed in terms of effective surface temperature as measured by a distant observer, $T_{\mathrm{s}}^{\infty}$. Curves 1 and 4 were obtained for pure crystal accreted crust with two different models of superfluidity of neutrons in the inner crust (strong and weak). Curves 1 and 4 give best fit to data points. Line 5 - amorphous ground state crust; line 2 - ground state crust without neutron superfluidity: both are ruled out by observations. From Shternin et al. [379]. Figure made by P.S. Shternin.

\section{Conclusion}

The conditions prevailing inside the crusts of neutron stars are not so extreme as those encountered in the dense core. Nonetheless, they are still far beyond those accessible in terrestrial laboratories. The matter in neutron star crust is subject to very high pressures, as well as huge magnetic fields, which can attain up to $10^{14}-10^{15} \mathrm{G}$ in magnetars. For comparison, it is worth reminding ourselves that the strongest (explosive) magnetic fields ever produced on the Earth reach "only" $3 \times 10^{7} \mathrm{G}$ [298]. The description of such environments requires the interplay of many different branches of physics, from nuclear physics to condensed matter and plasma physics.

Considerable progress in the microscopic modeling of neutron star crusts has been achieved during the last few years. Yet the structure and properties of the crust remain difficult to predict, depending on the formation and subsequent cooling of the star. Even in the ground state approximation, the structure of the neutron star crust is only well determined at $\rho \lesssim 10^{11} \mathrm{~g} \mathrm{~cm}^{-3}$, for which experimental data are available. Although all theoretical calculations predict the same large-scale picture of the denser layers of the crust, they do not quantitatively agree, reflecting the uncertainties in the properties of very exotic nuclei and uniform highly-asymmetric nuclear matter. The inner crust is expected to be formed of a lattice of neutron-rich nuclear clusters coexisting with a degenerate relativistic electron gas and a neutron liquid. The structure of the inner crust, its composition and the shape of the clusters are model dependent, especially in the bottom layers at densities $\sim 10^{14} \mathrm{~g} \mathrm{~cm}^{-3}$. However, the structure of the inner crust is crucial for calculating many properties, such as neutrino emissivities, as well as transport properties like electric and thermal conductivities. Superfluidity of unbound neutrons in the crust seems to be well established, both observationally and theoretically. However, much remains to be done to understand its properties in detail and, in particular, the effects of the nuclear lattice.

The interpretation of many observed neutron star phenomena, like pulsar glitches, X-ray bursts 
in low-mass X-ray binaries, initial cooling in soft X-ray transients, or quasi-periodic oscillations in soft gamma repeaters, can potentially shed light on the microscopic properties of the crust. However, their description requires consistent models of the crust from the nuclear scale up to the macroscopic scale. In particular, understanding the evolution of the magnetic field, the thermal relaxation of the star, the formation of mountains, the occurrence of starquakes and the propagation of seismic waves, requires the development of global models combining general relativity, elasticity, magnetohydrodynamics and superfluid hydrodynamics. Theoretical modeling of neutron star crusts is very challenging, but not out of reach. A confrontation of these models with observations could hopefully help us to unveil the intimate nature of dense matter at subnuclear densities. The improvement of observational techniques, as well as the development of gravitational wave astronomy in the near future, open very exciting perspectives.

We are aware that some aspects of the physics of neutron star crusts have not been dealt with in this review. Our intention was not to write an exhaustive monograph, but to give a glimpse of the great variety of topics that are necessarily addressed by different communities of scientists. We hope that this review will be useful to the reader for his/her own research. 


\section{Acknowledgments}

N.C. gratefully acknowledges financial support from a Marie Curie Intra-European grant (contract number MEIF-CT-2005-024660) and from FNRS (Belgium). P.H. was partially supported by the Polish MNiSW grant no. N20300632/0450.

We express our deep gratitude to D.G. Yakovlev for his help in the preparation of the present review. He read the whole manuscript and, during many hours of Skype sessions, he went over with one of us (P.H.) all the sections that required corrections, clarifications and improvement (they were legion). We thank him for his patience and expertise shared with us.

We are grateful to A.Y. Potekhin, who suggested several improvements in the sections referring to the effects of magnetic fields. He also suggested appropriate figures and made some new ones, which enriched the content of this review. Sometimes a good figure is better than hundreds of words.

We also thank Lars Samuelsson for his comments on elasticity and on axial modes in neutron stars.

We would like to address our gratitude to our colleagues, who contributed to this review through discussions or collaborations. 


\section{A List of Notations}

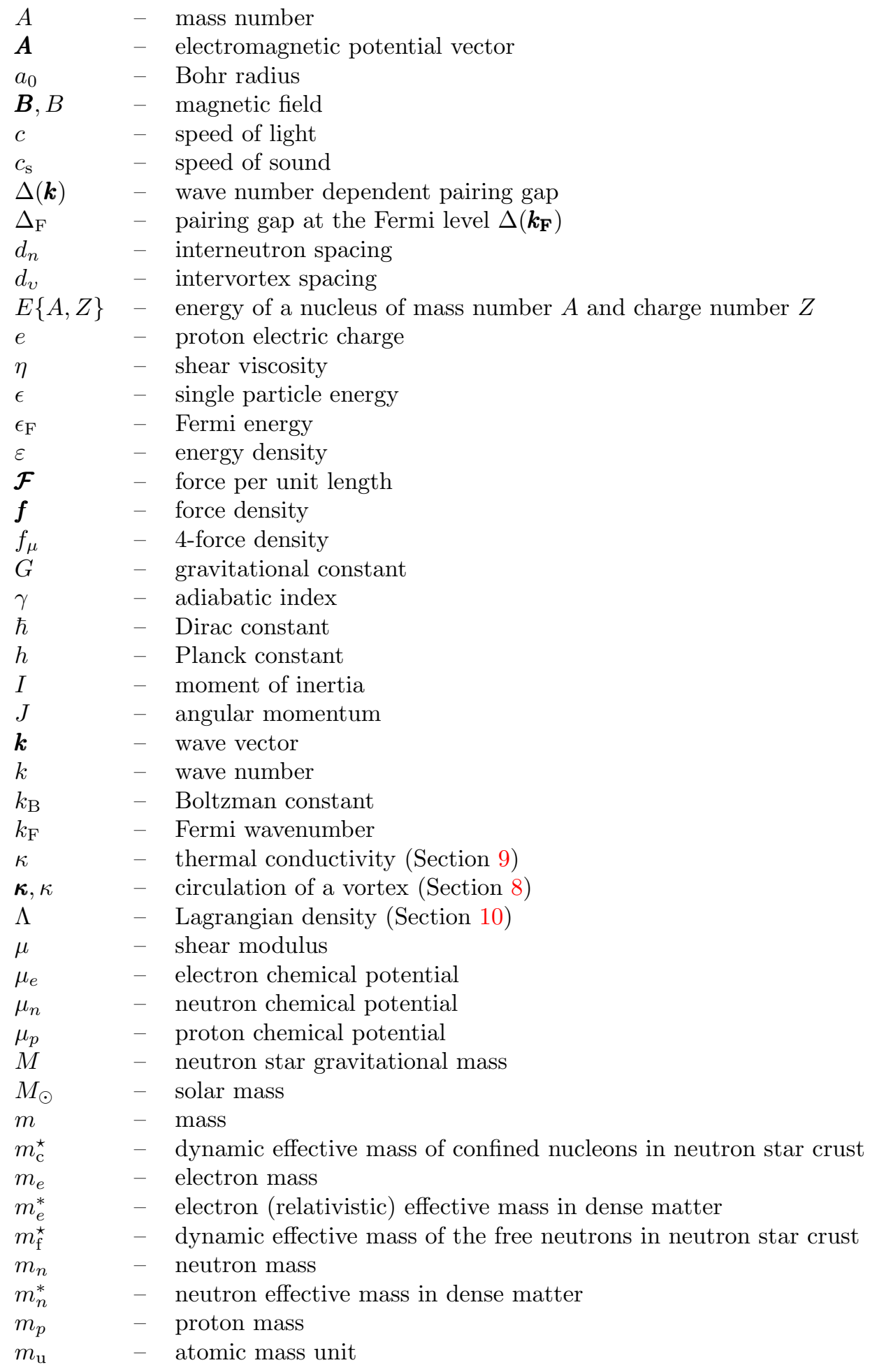




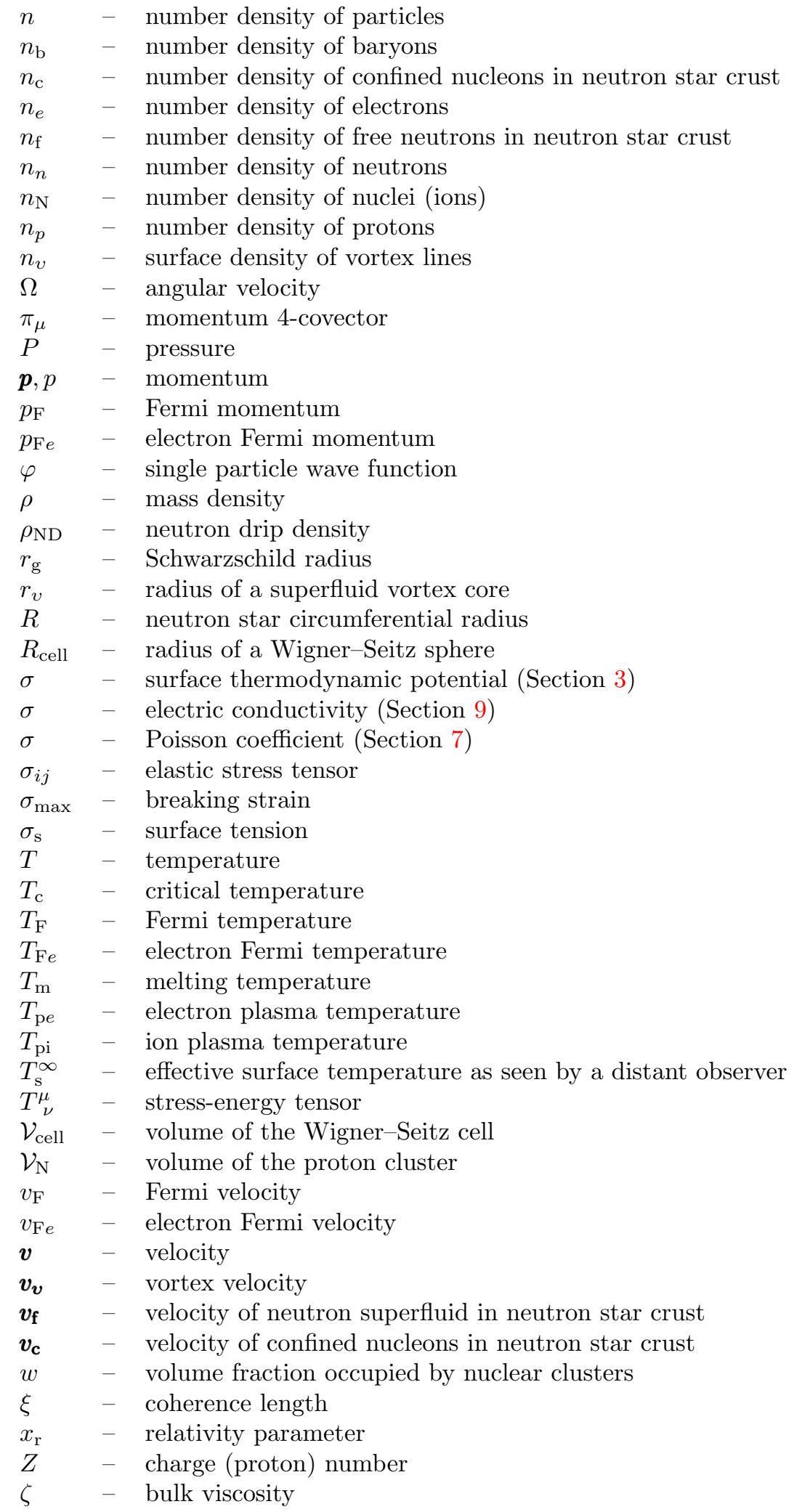




\section{B List of Abbreviations}

\begin{tabular}{|c|c|c|}
\hline bcc & - & body-centered cubic \\
\hline $\mathrm{BCS}$ & - & Bardeen-Cooper-Schrieffer \\
\hline $\mathrm{BE}$ & - & Boltzman equation \\
\hline $\mathrm{BEC}$ & - & Bose-Einstein condensate \\
\hline EoS & - & equation of state \\
\hline LMXB & - & low-mass X-ray binary \\
\hline QED & - & quantum electrodynamics \\
\hline $\mathrm{QPO}$ & - & Quasi Periodic Oscillation \\
\hline SGR & - & Soft Gamma Repeater \\
\hline $\mathrm{SXT}$ & - & Soft X-ray Transient \\
\hline W-S & - & Wigner-Seitz \\
\hline
\end{tabular}




\section{References}

[1] Abbott, B. et al. (LIGO Scientific Collaboration), "Upper limits on gravitational wave emission from 78 radio pulsars", Phys. Rev. D, 76(4), 042001, (2007). Related online version (cited on 2 October 2007): http://arXiv.org/abs/gr-qc/0702039. 12.5.1

[2] Abbott, B. et al. (LIGO Scientific Collaboration), "Beating the Spin-Down Limit on Gravitational Wave Emission from the Crab Pulsar", Astrophys. J. Lett., 683, L45-L49, (2008). Related online version (cited on 8 December 2008): http://arXiv.org/abs/0805.4758. 12.5.1, 78

[3] Abrikosov, A.A., Gorkov, L.P., and Dzyaloshinski, I.E., Methods of Quantum Field Theory in Statistical Physics, (Dover, New York, U.S.A., 1975), rev. edition. 8.2.1

[4] Alford, M., Bowers, J.A., and Rajagopal, K., "Crystalline color superconductivity", Phys. Rev. D, 63(7), 074016, (2001). Related online version (cited on 2 October 2007): http://arXiv.org/abs/hep-ph/0008208. 12.4.3

[5] Alpar, M.A., "Pinning and Threading of Quantized Vortices in the Pulsar Crust Superfluid", Astrophys. J., 213, 527-530, (1977). 8.3.5

[6] Alpar, M.A., Chau, H.F., Cheng, K.S., and Pines, D., "Postglitch relaxation of the VELA pulsar after its first eight large glitches: A reevaluation with the vortex creep model", Astrophys. J., 409, 345-359, (1993).

ADS: http://adsabs.harvard.edu/abs/1993ApJ . . 409. .345A. 12.4 .2

[7] Alpar, M.A., Chau, H.F., Cheng, K.S., and Pines, D., "Postglitch Relaxation of the Crab Pulsar after Its First Four Major Glitches: The Combined Effects of Crust Cracking, Formation of Vortex Depletion Region and Vortex Creep", Astrophys. J., 459, 706, (1996). 12.4 .2

[8] Alpar, M.A., Langer, S.A., and Sauls, J.A., "Rapid postglitch spin-up of the superfluid core in pulsars", Astrophys. J., 282, 533-541, (1984). 8.3.7

[9] Alpar, M.A., Pines, D., Anderson, P.W., and Shaham, J., "Vortex creep and the internal temperature of neutron stars. I. General theory", Astrophys. J., 276, 325-334, (1984).

ADS: http://adsabs.harvard.edu/abs/1984ApJ . . 276 . 325A. 12.4 .2

[10] Anderson, P.W., and Itoh, N., "Pulsar glitches and restlessness as a hard superfluidity phenomenon", Nature, 256, 25-27, (1975). 12.4, 12.4.2

[11] Anderson, P.W., and Morel, P., "Generalized Bardeen-Cooper-Schrieffer States and the Proposed Low-Temperature Phase of Liquid He ${ }^{3 "}$, Phys. Rev., 123, 1911-1934, (1961). 8.2.1

[12] Andersson, N., "Gravitational waves from instabilities in relativistic stars", Class. Quantum Grav., 20, R105-R144, (2003). Related online version (cited on 2 October 2007): http://arXiv.org/abs/astro-ph/0211057. 12.5.2

[13] Andersson, N., and Comer, G.L., "On the dynamics of superfluid neutron star cores", Mon. Not. R. Astron. Soc., 328, 1129-1143, (2001). Related online version (cited on 2 October 2007):

http://arXiv.org/abs/astro-ph/0101193. 12.6 
[14] Andersson, N., and Comer, G.L., "Probing Neutron-Star Superfluidity with GravitationalWave Data", Phys. Rev. Lett., 87, 241101, (2001). Related online version (cited on 2 October 2007):

http://arXiv.org/abs/gr-qc/0110112. 8.3.7

[15] Andersson, N., and Comer, G.L., "Relativistic Fluid Dynamics: Physics for Many Different Scales", Living Rev. Relativity, 10, lrr-2007-1, (2007). URL (cited on 2 October 2007): http://www.livingreviews.org/Irr-2007-1. 10, 10.1

[16] Andersson, N., Comer, G.L., and Prix, R., "The superfluid two-stream instability", Mon. Not. R. Astron. Soc., 354, 101-110, (2004). 12.4.3

[17] Andersson, N., and Kokkotas, K.D., "The R-Mode Instability in Rotating Neutron Stars", Int. J. Mod. Phys. D, 10, 381-441, (2001). Related online version (cited on 2 October 2007): http://arXiv.org/abs/gr-qc/0010102. 12.5.3

[18] Andersson, N., Sidery, T., and Comer, G.L., "Mutual friction in superfluid neutron stars", Mon. Not. R. Astron. Soc., 368, 162-170, (2006). Related online version (cited on 2 October 2007):

http://arXiv.org/abs/astro-ph/0510057. 8.3.5, 8.3.7

[19] Andersson, N., Sidery, T., and Comer, G.L., "Superfluid neutron star turbulence", Mon. Not. R. Astron. Soc., 381, 747-756, (2007). Related online version (cited on 16 June 2008): http://arXiv.org/abs/astro-ph/0703257. 8.3.5

[20] Andreev, A.F., and Bashkin, E.P., "Three-velocity hydrodynamics of superfluid solutions", Sov. Phys. JETP, 42, 164-167, (1975). 8.3.7, 10.2

[21] Andronikashvili, E.L., "Direct observation of two types of motion in helium II", Zh. Eksp. Teor. Fiz., 16, 780-785, (1946). 8.3.6

[22] Annett, J.F., Superconductivity, Superfluids and Condensates, Oxford Master Series in Condensed Matter Physics, (Oxford University Press, Oxford, U.K.; New York, U.S.A., 2004). 8

[23] Arnould, M., Goriely, S., and Takahashi, K., "The r-process of stellar nucleosynthesis: Astrophysics and nuclear physics achievements and mysteries", Phys. Rep., 450, 97-213, (2007). Related online version (cited on 2 October 2007): http://arXiv.org/abs/0705.4512. 12.3, 73

[24] ATNF/CSIRO, "ATNF Pulsar Catalogue: Glitch parameters", web interface to database. URL (cited on 10 September 2007): http://www.atnf.csiro.au/research/pulsar/psrcat/glitchTbl.html. 12.4

[25] Audi, G., Wapstra, A.H., and Thibault, C., "The AME2003 atomic mass evaluation (II). Tables, graphs and references", Nucl. Phys. A, 729, 337-676, (2003). ADS: http://adsabs.harvard.edu/abs/2003NuPhA.729. .337A. 3.1

[26] Australia Telescope National Facility, "ATNF pulsar database", web interface to database. URL (cited on 20 August 2007): http://www .atnf.csiro.au/research/pulsar/psrcat/. 12.4

[27] Avogadro, P., Barranco, F., Broglia, R.A., and Vigezzi, E., "Quantum calculation of vortices in the inner crust of neutron stars", Phys. Rev. C, 75, 012805, (2007). Related online version (cited on 2 October 2007): http://arXiv.org/abs/nucl-th/0602028. 8.3.5, 8.3.5 
[28] Baldo, M., Lombardo, U., Saperstein, E.E., and Tolokonnikov, S.V., "The role of superfluidity in the structure of the neutron star inner crust", Nucl. Phys. A, 750, 409-424, (2005). 3.2.3

[29] Baldo, M., Saperstein, E.E., and Tolokonnikov, S.V., "Superfluidity in nuclear and neutron matter", Nucl. Phys. A, 749, 42-52, (2005). 3.2.3, 3.2.3, 8, 8.2, 8.2.3

[30] Baldo, M., Saperstein, E.E., and Tolokonnikov, S.V., "The role of the boundary conditions in the Wigner Seitz approximation applied to the neutron star inner crust", Nucl. Phys. A, 775, 235-244, (2006). Related online version (cited on 2 October 2007): http://arXiv.org/abs/nucl-th/0605010. 3.2.3, 3.2.3, 3.2.4, 8.2.3

[31] Baldo, M., Saperstein, E.E., and Tolokonnikov, S.V., "A realistic model of superfluidity in the neutron star inner crust", Eur. Phys. J. A, 32, 97-108, (2007). Related online version (cited on 2 October 2007): http://arXiv.org/abs/nucl-th/0609031. 11, 3.2.3, 4, 3.2.4, 8.2.3

[32] Baldo, M., Saperstein, E.E., and Tolokonnikov, S.V., "Upper edge of the neutron star inner crust: The drip point and its vicinity", Phys. Rev. C, 76, 025803, (2007). Related online version (cited on 16 June 2008):

http://arXiv.org/abs/nucl-th/0703099. 11, 3.2.3, 4, 49

[33] Balibar, S., "The Discovery of Superfluidity", J. Low Temp. Phys., 146, 441-470, (2007). 8.3.6

[34] Barat, C., Hayles, R.I., Hurley, K., Niel, M., Vedrenne, G., Desai, U., Kurt, V.G., Zenchenko, V.M., and Estulin, I.V., "Fine time structure in the 1979 March 5 gamma ray burst", Astron. Astrophys., 126, 400-402, (1983). 12.6

[35] Bardeen, J., "Critical Fields and Currents in Superconductors", Rev. Mod. Phys., 34, 667681, (1962). 8.3.1

[36] Bardeen, J., Cooper, L.N., and Schrieffer, J.R., "Theory of Superconductivity", Phys. Rev., 108, 1175-1204, (1957). 8.1, 8.1, 8.2, 8.2.2

[37] Barranco, F., Broglia, R.A., Esbensen, H., and Vigezzi, E., "Role of finite nuclei on the pairing gap of the inner crust of neutron stars", Phys. Lett. B, 390, 13-17, (1997). 8.2.3

[38] Barranco, F., Broglia, R.A., Esbensen, H., and Vigezzi, E., "Semiclassical approximation to neutron star superfluidity corrected for proximity effects", Phys. Rev. C, 58, 1257-1262, (1998). Related online version (cited on 2 October 2007): http://arXiv.org/abs/nucl-th/9806056. 8.2.3

[39] Baym, G., Bethe, H.A., and Pethick, C.J., "Neutron Star Matter", Nucl. Phys. A, 175, 225-271, (1971). 3.2.1, 3.2.1, 3.2.1, 3.3

[40] Baym, G., Pethick, C., Pines, D., and Ruderman, M., "Spin Up in Neutron Stars: The Future of the Vela Pulsar", Nature, 224, 872, (1969). 12.4.2

[41] Baym, G., Pethick, C.J., and Pines, D., "Superfluidity in Neutron Stars", Nature, 224, 673, (1969). 8.2, 8.3.4, 12.4

[42] Baym, G., Pethick, C.J., and Sutherland, P., "The Ground State of Matter at High Densities: Equation of State and Stellar Models", Astrophys. J., 170, 299-317, (1971). 3.1, 3.1, 3.2, $5.1,5$ 
[43] Baym, G., and Pines, D., "Neutron starquakes and pulsar speedup.", Ann. Phys. (N.Y.), 66, 816-835, (1971). 7.1

[44] Beig, R., and Schmidt, B.G., "Relativistic elasticity", Class. Quantum Grav., 20, 889-904, (2003). Related online version (cited on 2 October 2007): http://arXiv.org/abs/gr-qc/0211054. 7

[45] Bejger, M., Haensel, P., and Zdunik, J.L., "Rotation at $1122 \mathrm{~Hz}$ and the neutron star structure", Astron. Astrophys., 464, L49-L52, (2007). Related online version (cited on 2 October 2007):

http://arXiv.org/abs/astro-ph/0612216. 12.7.1

[46] Belyaev, S.T., "Effect of pairing correlations on nuclear properties", Mat.-Fys. Medd. K. Dan. Vid. Selsk., 31(11), 1-55, (1959). 8.2

[47] Bender, M., Heenen, P., and Reinhard, P., "Self-consistent mean-field models for nuclear structure", Rev. Mod. Phys., 75, 121-180, (2003). 3.2.4

[48] Bildsten, L., and Epstein, R.I., "Superfluid dissipation time scales in neutron star crusts", Astrophys. J., 342, 951-957, (1989). 8.3.5

[49] Bildsten, L., and Ushomirsky, G., "Viscous Boundary-Layer Damping of $r$-Modes in Neutron Stars", Astrophys. J., 529, L33-L36, (2000). Related online version (cited on 2 October 2007): http://arXiv.org/abs/astro-ph/9911155. 12.5.3

[50] Bisnovatyi-Kogan, G.S., and Chechetkin, V.M., "Nonequilibrium envelopes of the neutron stars, their role in the maintenance of X-ray luminosity and nucleosynthesis", Usp. Fiz. Nauk, 127, 263-296, (1979). 4.3

[51] Bohr, A., Mottelson, B.R., and Pines, D., "Possible Analogy between the Excitation Spectra of Nuclei and Those of the Superconducting Metallic State", Phys. Rev., 110, 936-938, (1958). 8.2

[52] Bohr, N., and Wheeler, J.A., "The Mechanism of Nuclear Fission", Phys. Rev., 56, 426-450, (1939). 3.3

[53] Bonazzola, S., Gourgoulhon, E., Salgado, M., and Marck, J.A., "Axisymmetric rotating relativistic bodies: A new numerical approach for 'exact' solutions", Astron. Astrophys., 278, 421-443, (1993). 6.3

[54] Bonche, P., and Vautherin, D., "A mean-field calculation of the equation of state of supernova matter.", Nucl. Phys. A, 372, 496-526, (1981). 3.2.4

[55] Bonche, P., and Vautherin, D., "Mean-field calculations of the equation of state of supernova matter II", Astron. Astrophys., 112, 268-272, (1982). 3.2.4, 3.4

[56] Botvina, A.S., and Mishustin, I.N., "Multifragmentation reactions and properties of stellar matter at subnuclear densities", Phys. Rev. C, 72, 048801, (2005). Related online version (cited on 2 October 2007):

http://arXiv.org/abs/nucl-th/0506061. 3.3

[57] Botvina, A.S., Mishustin, I.N., and Trautmann, W., "Properties of stellar matter in supernova explosions and nuclear multifragmentation", (2006). URL (cited on 2 October 2007): http://arXiv.org/abs/nucl-th/0612055. 3.3 
[58] Brack, M., Guet, C., and Hakansson, H.-B., "Selfconsistent semiclassical description of average nuclear properties-a link between microscopic and macroscopic models", Phys. Rep., 123, 275-364, (1985). 3.2.2

[59] Broglia, R.A., de Blasio, F., Lazzari, G., Lazzari, M., and Pizzochero, P.M., "Specific heat of superfluid matter in the inner crust of neutron stars", Phys. Rev. D, 50, 4781-4785, (1994). 8.2 .3

[60] Brown, E.F., Bildsten, L., and Rutledge, R.E., "Crustal Heating and Quiescent Emission from Transiently Accreting Neutron Stars", Astrophys. J. Lett., 504, L95, (1998). Related online version (cited on 2 October 2007):

http://arXiv.org/abs/astro-ph/9807179. 12.7.2

[61] Buchler, J.-R., and Barkat, Z., "Properties of low-density neutron-star matter.", Phys. Rev. Lett., 27, 48-51, (1971). 10

[62] Bulgac, A., and Magierski, P., "Quantum corrections to the ground state energy of inhomogeneous neutron matter", Nucl. Phys. A, 683, 695-712, (2001). Related online version (cited on 2 October 2007):

http://arXiv.org/abs/astro-ph/0002377. 3.2.2

[63] Burrows, A., and Lattimer, J.M., "On the accuracy of the single-nucleus approximation in the equation of state of hot, dense matter", Astrophys. J., 285, 294-303, (1984). 3.4

[64] Bürvenich, T.J., Mishustin, I.N., and Greiner, W., "Nuclei embedded in an electron gas", Phys. Rev. C, 76, 034310, (2007). Related online version (cited on 16 June 2008): http://arXiv.org/abs/0706.1450. 3.2.4

[65] Caballero, O.L., Horowitz, C.J., and Berry, D.K., "Neutrino scattering in heterogeneous supernova plasmas", Phys. Rev. C, 74(6), 065801, (2006). Related online version (cited on 2 October 2007):

http://arXiv.org/abs/astro-ph/0607564. 12.1

[66] Cackett, E.M., Wijnands, R., Linares, M., Miller, J.M., Homan, J., and Lewin, W.H.G., "Cooling of the quasi-persistent neutron star X-ray transients KS 1731-260 and MXB 165929", Mon. Not. R. Astron. Soc., 372, 479-488, (2006). Related online version (cited on 2 October 2007): http://arXiv.org/abs/astro-ph/0605490. 12.7.3

[67] California Institute of Technology, "LIGO Laboratory Home Page", project homepage. URL (cited on 20 August 2007): http://www.ligo.caltech.edu/. 12.5

[68] Campbell, L.J., "Rotational speedups accompanying angular deceleration of a superfluid", Phys. Rev. Lett., 43, 1336-1339, (1979). 12.4

[69] Cao, L.G., Lombardo, U., and Schuck, P., "Screening effects in superfluid nuclear and neutron matter within Brueckner theory", Phys. Rev. C, 74(6), 064301, (2006). Related online version (cited on 2 October 2007): http://arXiv.org/abs/nucl-th/0608005. 5, 8.2.2, 47, 48

[70] Carter, B., "Covariant theory of conductivity in ideal fluid or solid media", in Anile, A., and Choquet-Bruhat, Y., eds., Relativistic Fluid Dynamics, Lectures given at the 1st 1987 Session of the Centro Internazionale Matematico Estivo (C.I.M.E.) held at Noto, Italy, May 25 -June 3, 1987, Lecture Notes in Mathematics, vol. 1385, pp. 1-64, (Springer, Berlin, Germany; New York, U.S.A., 1989). 1, 6, 10.1 
[71] Carter, B., "Relativistic dynamics of vortex defects in superfluids", in Bunkov, Y.M., and Godfrin, H., eds., Topological Defects and Non-Equilibrium Dynamics of Phase Transitions, Proceedings of the Les Houches Winter School, 16-26 February 1999, NATO Science Series C, vol. 549, pp. 267-302, (Kluwer Academic Publishers, Dordrecht, Netherlands; Boston, U.S.A., 2000). 8.3 .5

[72] Carter, B., and Chachoua, E., "Newtonian Mechanics of Neutron Superfluid in Elastic Star Crust", Int. J. Mod. Phys. D, 15, 1329-1358, (2006). Related online version (cited on 2 October 2007):

http://arXiv.org/abs/astro-ph/0601658. 7, 10, 10.1, 12.6

[73] Carter, B., Chachoua, E., and Chamel, N., "Covariant Newtonian and relativistic dynamics of (magneto)-elastic solid model for neutron star crust", Gen. Relativ. Gravit., 38, 83-119, (2006). Related online version (cited on 2 October 2007): http://arXiv.org/abs/gr-qc/0507006. 7, 10, 10.1, 12.6

[74] Carter, B., and Chamel, N., "Covariant Analysis of Newtonian Multi-Fluid Models for Neutron Stars I: Milne-Cartan Structure and Variational Formulation", Int. J. Mod. Phys. D, 13, 291-325, (2004). Related online version (cited on 2 October 2007): http://arXiv.org/abs/astro-ph/0305186. 10, 10.1, 10.4

[75] Carter, B., and Chamel, N., "Covariant Analysis of Newtonian Multi-Fluid Models for Neutron Stars II: Stress-Energy Tensors and Virial Theorems", Int. J. Mod. Phys. D, 14, 717-748, (2005). Related online version (cited on 2 October 2007): http://arXiv.org/abs/astro-ph/0312414. 8.3.5, 10, 10.1, 10.4

[76] Carter, B., and Chamel, N., "Covariant Analysis of Newtonian Multi-Fluid Models for Neutron Stars III: Transvective, Viscous, and Superfluid Drag Dissipation", Int. J. Mod. Phys. $D, \mathbf{1 4}, 749-774,(2005)$. Related online version (cited on 2 October 2007): http://arXiv.org/abs/astro-ph/0410660. 10, 10.1, 10.2, 10.2, 10.2

[77] Carter, B., Chamel, N., and Haensel, P., "Effect of BCS pairing on entrainment in neutron superfluid current in neutron star crust", Nucl. Phys. A, 759, 441-464, (2005). Related online version (cited on 2 October 2007): http://arXiv.org/abs/astro-ph/0406228. 3.2.4, 8.3.1, 10

[78] Carter, B., Chamel, N., and Haensel, P., "Entrainment coefficient and effective mass for conduction neutrons in neutron star crust: simple microscopic models", Nucl. Phys. A, 748, 675-697, (2005). Related online version (cited on 2 October 2007): http://arXiv.org/abs/nucl-th/0402057. 3.2.4, 3.2.4, 8.3.7, 8.3.7, 10, 10.2

[79] Carter, B., Chamel, N., and Haensel, P., "Entrainment Coefficient and Effective Mass for Conduction Neutrons in Neutron Star Crust: Macroscopic Treatment", Int. J. Mod. Phys. $D, \mathbf{1 5}, 777-803,(2006)$. Related online version (cited on 2 October 2007): http://arXiv.org/abs/astro-ph/0408083. 8.3.7, 8.3.7, 10, 10.2, 10.2, 10.3, 10.4

[80] Carter, B., and Langlois, D., "Relativistic models for superconducting-superfluid mixtures", Nucl. Phys. B, 531, 478-504, (1998). Related online version (cited on 2 October 2007): http://arXiv.org/abs/gr-qc/9806024. 6, 10.1

[81] Carter, B., Langlois, D., and Sedrakian, D.M., "Centrifugal buoyancy as a mechanism for neutron star glitches", Astron. Astrophys., 361, 795-802, (2000). Related online version (cited on 2 October 2007): http://arXiv.org/abs/astro-ph/0004121. 12.4.3 
[82] Carter, B., and Quintana, H., "Foundations of General Relativistic High-Pressure Elasticity Theory", Proc. R. Soc. London, Ser. A, 331, 57-83, (1972). 7

[83] Carter, B., and Quintana, H., "Relativistic formulation of the neutron starquake theory of pulsar glitches", Ann. Phys. (N.Y.), 95, 74-89, (1975). 7

[84] Carter, B., and Quintana, H., "Stationary elastic rotational deformation of a relativistic neutron star model", Astrophys. J., 202, 511-522, (1975). 7

[85] Carter, B., and Samuelsson, L., "Relativistic mechanics of neutron superfluid in (magneto)elastic star crust", Class. Quantum Grav., 23, 5367-5388, (2006). Related online version (cited on 2 October 2007):

http://arXiv.org/abs/gr-qc/0605024. 7, 10, 10.1, 12.6

[86] Chabanat, E., Bonche, P., Haensel, P., Meyer, J., and Schaeffer, R., "A Skyrme parametrization from subnuclear to neutron star densities", Nucl. Phys. A, 627, 710-746, (1997). 5.1

[87] Chabanat, E., Bonche, P., Haensel, P., Meyer, J., and Schaeffer, R., "Erratum to 'A Skyrme parametrization from subnuclear to neutron star densities. Part II. Nuclei far from stabilities' [Nucl. Phys. A 635 (1998) 231-256]", Nucl. Phys. A, 643, 441-441, (1998). 5.1

[88] Chabanat, E., Bonche, P., Haensel, P., Meyer, J., and Schaeffer, R., "A Skyrme parametrization from subnuclear to neutron star densities. Part II. Nuclei far from stabilities", Nucl. Phys. A, 635, 231-256, (1998). 5.1

[89] Chakrabarty, D., Morgan, E.H., Muno, M.P., Galloway, D.K., Wijnands, R., van der Klis, M., and Markwardt, C.B., "Nuclear-powered millisecond pulsars and the maximum spin frequency of neutron stars", Nature, 424, 42-44, (2003). Related online version (cited on 2 October 2007):

http: //arXiv.org/abs/astro-ph/0307029. 12.7.1

[90] Chamel, N., "Band structure effects for dripped neutrons in neutron star crust", Nucl. Phys. A, 747, 109-128, (2005). Related online version (cited on 2 October 2007): http://arXiv.org/abs/nucl-th/0405003. 3.2.2, 3.2.4, 3.2.4, 3.2.4, 8.3.1, 8.3.7, 10.2, 10.3, 12.4.4, 12.4.4, 12.4.4, 12.6

[91] Chamel, N., "Effective mass of free neutrons in neutron star crust", Nucl. Phys. A, 773, 263-278, (2006). Related online version (cited on 2 October 2007):

http://arXiv.org/abs/nucl-th/0512034. 3.2.4, 3.2.4, 3.2.4, 8.3.1, 10.3, 12.4.4, 12.4.4, 12.6

[92] Chamel, N., "Neutron Star Crust beyond the Wigner-Seitz Approximation", in Lombardo, U., Baldo, M., Burgio, F., and Schulze, H.-J., eds., Exotic States of Nuclear Matter, Proceedings of the International Symposium EXOCT07, Catania, Italy, June 11 - June 15, 2007, pp. 91-98, (World Scientific, Singapore; Hackensack, U.S.A., 2008). Related online version (cited on 30 April 2008):

http://arXiv.org/abs/0709.3798. 3.2.3

[93] Chamel, N., "Two-fluid models of superfluid neutron star cores", Mon. Not. R. Astron. Soc., 388, 737-752, (2008). Related online version (cited on 8 December 2008):

http://arXiv.org/abs/0805.1007. 10, 10.2, 10.3, 10.3

[94] Chamel, N., and Carter, B., "Effect of entrainment on stress and pulsar glitches in stratified neutron star crust", Mon. Not. R. Astron. Soc., 368, 796-808, (2006). Related online version 
(cited on 2 October 2007):

http://arXiv.org/abs/astro-ph/0503044. 10, 10.2, 10.2, 10.4, 12.4.3, 12.4.4, 12.4.4, $12.4 .4,12.4 .4$

[95] Chamel, N., and Haensel, P., "Entrainment parameters in a cold superfluid neutron star core", Phys. Rev. C, 73, 045802, (2006). Related online version (cited on 2 October 2007): http://arXiv.org/abs/nucl-th/0603018. 10.3

[96] Chamel, N., Naimi, S., Khan, E., and Margueron, J., "Validity of the Wigner-Seitz approximation in neutron star crust", Phys. Rev. C, 75, 055806, (2007). Related online version (cited on 2 October 2007):

http://arXiv.org/abs/astro-ph/0701851. 3.2.4, 15

[97] Cheng, K.S., Yao, C.C., and Dai, Z.G., "Properties of nuclei in the inner crusts of neutron stars in the relativistic mean-field theory", Phys. Rev. C, 55, 2092-2100, (1997). 3.3

[98] Chiu, H.-Y., and Morrison, P., "Neutrino Emission from Black-Body Radiation at High Stellar Temperatures", Phys. Rev. Lett., 5, 573-575, (1960). 11.2

[99] Chiu, H.-Y., and Stabler, R.C., "Emission of Photoneutrinos and Pair Annihilation Neutrinos from Stars", Phys. Rev., 122, 1317-1322, (1961). 11.4

[100] Chugunov, A.I., and Haensel, P., "Thermal conductivity of ions in a neutron star envelope", Mon. Not. R. Astron. Soc., 381, 1143-1153, (2007). Related online version (cited on 12 June 2008):

http://arXiv.org/abs/0707.4614. 9.3, 9.5.4

[101] Chugunov, A.I., and Yakovlev, D.G., "Shear Viscosity and Oscillations of Neutron Star Crust", Astron. Rep., 49, 724-738, (2005). Related online version (cited on 2 October 2007): http://arXiv.org/abs/astro-ph/0511300. 56, 9.4

[102] Colpi, M., Geppert, U., Page, D., and Possenti, A., "Charting the Temperature of the Hot Neutron Star in a Soft X-Ray Transient", Astrophys. J., 548, L175-L178, (2001). Related online version (cited on 2 October 2007):

http://arXiv.org/abs/astro-ph/0010572. 12.7.2

[103] Combescot, R., "Ultracold Fermi Gases: The BEC-BCS Crossover", J. Low Temp. Phys., 145, 267-276, (2006). 8.1

[104] Cumming, A., Macbeth, J., in 't Zand, J.J.M., and Page, D., "Long Type I X-Ray Bursts and Neutron Star Interior Physics", Astrophys. J., 646, 429-451, (2006). Related online version (cited on 2 October 2007):

http://arXiv.org/abs/astro-ph/0508432. 12.7

[105] Cutler, C., "Gravitational waves from neutron stars with large toroidal B fields", Phys. Rev. D, 66, 084025, (2002). Related online version (cited on 26 May 2008):

http://arXiv.org/abs/gr-qc/0206051. 12.5.1

[106] Dall'Osso, S., Israel, G.L., Stella, L., Possenti, A., and Perozzi, E., "The Glitches of the Anomalous X-Ray Pulsar 1RXS J170849.0-400910", Astrophys. J., 599, 485-497, (2003). 12.4

[107] de Blasio, F., Lazzari, G., Pizzochero, P.M., and Broglia, R.A., "Superfluidity and thermal response of neutron star crusts", Phys. Rev. D, 53, 4226-4231, (1996). 8.2.3 
[108] de Blasio, F.V., "Crustal impurities and the internal temperature of a neutron star crust", Mon. Not. R. Astron. Soc., 299, 118-122, (1998). 3.4

[109] de Blasio, F.V., "A dense two-component plasma in a strong gravity field and thermal conductivity of neutron stars", Astron. Astrophys., 353, 1129-1133, (2000). 3.4

[110] de Blasio, F.V., and Elgarøy, Ø., "Microscopic Structure of a Vortex Line in Superfluid Neutron Star Matter", Phys. Rev. Lett., 82, 1815-1818, (1999). Related online version (cited on 2 October 2007): http://arXiv.org/abs/nucl-th/9808057. 8.3.2

[111] de Blasio, F.V., Hjorth-Jensen, M., Elgarøy, Ø., Engvik, L., Lazzari, G., Baldo, M., and Schulze, H.-J., "Coherence length of neutron superfluids", Phys. Rev. C, 56, 2332-2335, (1997). 8.2.3

[112] de Blasio, F.V., and Lazzari, G., "Unusual nuclear shapes and neutron specific heat of neutron star crusts", Phys. Rev. C, 52, 418-420, (1995). 3.3

[113] de Blasio, F.V., and Lazzari, G., "Vibrations of slabs and cylindrical nuclei", Phys. Lett. B, 384, 1-4, (1996). 3.3

[114] de Gennes, P.G., Superconductivity of Metals and Alloys, (W.A. Benjamin, New York, U.S.A., 1966). 8.2.3

[115] de Gennes, P.G., and Prost, J., The Physics of Liquid Crystals, The International Series of Monographs on Physics, vol. 83, (Clarendon Press; Oxford University Press, Oxford, U.K.; New York, U.S.A., 1993), 2nd edition. 7.2, 7.2

[116] Dean, D.J., and Hjorth-Jensen, M., "Pairing in nuclear systems: from neutron stars to finite nuclei", Rev. Mod. Phys., 75, 607-656, (2003). 8, 8.2

[117] Dechargé, J., and Gogny, D., "Hartree-Fock-Bogolyubov calculations with the D1 effective interaction on spherical nuclei", Phys. Rev. C, 21, 1568-1593, (1980).

ADS: http://adsabs.harvard.edu/abs/1980PhRvC. .21.1568D. 8.2.1

[118] Department of Theoretical Astrophysics, Ioffe Physico Technical-Institute, "Neutron Star Group", project homepage. URL (cited on 20 August 2007):

http://www.ioffe.ru/astro/NSG/. 5.1

[119] Dobaczewski, J., Michel, N., Nazarewicz, W., Płoszajczak, M., and Rotureau, J., "Shell structure of exotic nuclei", Prog. Part. Nucl. Phys., 59, 432-445, (2007). Related online version (cited on 2 October 2007):

http://arXiv.org/abs/nucl-th/0701047. 3.2.3

[120] Donati, P., and Pizzochero, P.M., "Is there Nuclear Pinning of Vortices in Superfluid Pulsars?", Phys. Rev. Lett., 90, 211101, (2003). 8.3.5

[121] Donati, P., and Pizzochero, P.M., "Fully consistent semi-classical treatment of vortex-nucleus interaction in rotating neutron stars", Nucl. Phys. A, 742, 363-379, (2004). 8.3.5

[122] Donati, P., and Pizzochero, P.M., "Realistic energies for vortex pinning in intermediatedensity neutron star matter", Phys. Lett. B, 640, 74-81, (2006). 8.3.5

[123] Douchin, F., and Haensel, P., "Bounds on the Existence of Neutron Rich Nuclei in Neutron Star Interiors", Acta Phys. Pol. B, 30, 1205, (1999). Related online version (cited on 2 October 2007): http://arXiv.org/abs/astro-ph/9808030. 6 
[124] Douchin, F., and Haensel, P., "Inner edge of neutron-star crust with SLy effective nucleonnucleon interactions", Phys. Lett. B, 485, 107-114, (2000). Related online version (cited on 2 October 2007):

http://arXiv.org/abs/astro-ph/0006135. 7, 8, 9, 3.3, 3.3

[125] Douchin, F., and Haensel, P., "A unified equation of state of dense matter and neutron star structure", Astron. Astrophys., 380, 151-167, (2001). 3, 3.2.1, 19, 32, 37, 40, 42

[126] Douchin, F., Haensel, P., and Meyer, J., "Nuclear surface and curvature properties for SLy Skyrme forces and nuclei in the inner neutron-star crust", Nucl. Phys. A, 665, 419-446, (2000). 3.2.1, 5.1, 43

[127] Duine, R.A., and Stoof, H.T.C., "Atom-molecule coherence in Bose gases", Phys. Rep., 396, 115-195, (2004). Related online version (cited on 24 April 2008): http://arXiv.org/abs/cond-mat/0312254. 8.2.1

[128] Duncan, R.C., "'Magnetars', soft gamma repeaters and very strong magnetic fields", personal homepage, University of Texas Austin. URL (cited on 20 August 2007):

http://solomon.as.utexas.edu/ duncan/magnetar.html. 12.6

[129] Duncan, R.C., "Global Seismic Oscillations in Soft Gamma Repeaters", Astrophys. J. Lett., 498, L45, (1998). Related online version (cited on 2 October 2007): http: //arXiv.org/abs/astro-ph/9803060. 12.6, 12.6

[130] Dutta, A.K., Onsi, M., and Pearson, J.M., "Proton-shell effects in neutron-star matter", Phys. Rev. C, 69, 052801, (2004). 3.2.2

[131] Dyson, F.J., "Volcano Theory of Pulsars", Nature, 223, 486-487, (1969). 12.3

[132] Easson, I., "Long-term changes in pulsar periods and the plasma in neutron star interiors", Astrophys. J., 233, 711-716, (1979). 12.4.2

[133] Easson, I., "Postglitch behavior of the plasma inside neutron stars", Astrophys. J., 228, 257-267, (1979). 12.4.2

[134] Elgarøy, Ø., and de Blasio, F.V., "Superfluid vortices in neutron stars", Astron. Astrophys., 370, 939-950, (2001). Related online version (cited on 2 October 2007): http://arXiv.org/abs/astro-ph/0102343. 8.3.2, 8.3.5, 8.3.5

[135] Elgarøy, Ø., Engvik, L., Osnes, E., de Blasio, F.V., Hjorth-Jensen, M., and Lazzari, G., "Superfluidity and neutron star crust matter", Phys. Rev. D, 54, 1848-1851, (1996). Related online version (cited on 2 October 2007):

http://arXiv.org/abs/nucl-th/9509035. 3.3, 8.2.3

[136] Elgarøy, Ø., and Hjorth-Jensen, M., "Nucleon-nucleon phase shifts and pairing in neutron matter and nuclear matter", Phys. Rev. C, 57, 1174-1177, (1998). Related online version (cited on 2 October 2007):

http://arXiv.org/abs/nucl-th/9708026. 8.2.1, 8.2.1

[137] Ellis, T., and McClintock, P.V.E., "The Breakdown of Superfluidity in Liquid ${ }^{4} H e$ V. Measurement of the Landau Critical Velocity for Roton Creation", Philos. Trans. R. Soc. London, Ser. A, 315, 259-300, (1985). 8.3.1

[138] Epstein, R.I., and Baym, G., "Vortex pinning in neutron stars", Astrophys. J., 328, 680-690, (1988). 8.3.5 
[139] Epstein, R.I., and Baym, G., "Vortex drag and the spin-up time scale for pulsar glitches", Astrophys. J., 387, 276-287, (1992). 8.3.5

[140] Farine, M., Von-Eiff, D., Schuck, P., Berger, J.F., Dechargé, J., and Girod, M., "Towards a new effective interaction of the Gogny type", J. Phys. G, 25, 863-866, (1999). 8.2.1

[141] Feibelman, P.J., "Relaxation of Electron Velocity in a Rotating Neutron Superfluid: Application to the Relaxation of a Pulsar's Slowdown Rate", Phys. Rev. D, 4, 1589-1597, (1971). 8.3 .5

[142] Fetter, A.L., and Walecka, J.D., Quantum Theory of Many-Particle Systems, (Dover, Mineola, U.S.A., 2003). 8.3.1

[143] Feynman, R.P., "Application of quantum mechanics to liquid helium", in Gorter, C.J., ed., Progress in Low Temperature Physics, vol. 1, p. 17, (North-Holland, Amsterdam, Netherlands, 1955). 8.3.1, 8.3.2

[144] Feynman, R.P., Statistical Mechanics: A Set Of Lectures, Advanced Book Classics, (Westview Press, Boulder, U.S.A., 1998). 8.3.6

[145] Feynman, R.P., and Cohen, M., "Energy Spectrum of the Excitations in Liquid Helium", Phys. Rev., 102, 1189-1204, (1956). 8.3.1

[146] Flowers, E., and Itoh, N., "Transport properties of dense matter", Astrophys. J., 206, 218$242,(1976) .9 .4$

[147] Flowers, E., and Itoh, N., "Transport properties of dense matter. II", Astrophys. J., 230, 847-858, (1979). 9.4

[148] Flowers, E., Ruderman, M., and Sutherland, P., "Neutrino pair emission from finitetemperature neutron superfluid and the cooling of young neutron stars", Astrophys. J., 205, $541-544,(1976) .11 .6$

[149] Franco, L.M., Link, B., and Epstein, R.I., "Quaking Neutron Stars", Astrophys. J., 543, 987-994, (2000). Related online version (cited on 2 October 2007): http://arXiv.org/abs/astro-ph/9911105. 12.4.3

[150] Freedman, D.Z., "Coherent effects of a weak neutral current", Phys. Rev. D, 9, 1389-1392, (1974). 12.1

[151] Fuchs, K., "A Quantum Mechanical Calculation of the Elastic Constants of Monovalent Metals", Proc. R. Soc. London, Ser. A, 153, 622-639, (1936). 7.1

[152] Fujimoto, M.Y., Hanawa, T., and Miyaji, S., "Shell flashes on accreting neutron stars and X-ray bursts", Astrophys. J., 247, 267-278, (1981). 4.4

[153] Galloway, D.K., Morgan, E.H., and Levine, A.M., "A Frequency Glitch in an Accreting Pulsar", Astrophys. J., 613, 1164-1172, (2004). Related online version (cited on 2 October 2007):

http://arXiv.org/abs/astro-ph/0401476. 12.4

[154] Gandelman, G.M., and Pinaev, V.S., "Emission of neutrino pairs by electrons and its importance in stars", Zh. Eksp. Teor. Fiz., 37, 1072-1078, (1959). In Russian. 11.5

[155] Gandelman, G.M., and Pinaev, V.S., "Emission of neutrino pairs by electrons and its importance in stars", Sov. Phys. JETP, 10, 764, (1960). 11.5 
[156] Gelfand, J.D., "The radio nebula produced by the 27 December 2004 giant flare from SGR 1806-20", Astrophys. Space Sci., 308, 39-42, (2007).

ADS: http://adsabs.harvard.edu/abs/2007Ap\%26SS.308. . 39G. 12.3

[157] Gelfert, A., and Nolting, W., "The absence of finite-temperature phase transitions in lowdimensional many-body models: a survey and new results", J. Phys.: Cond. Matter, 13, R505-R524, (2001). Related online version (cited on 26 May 2008):

http://arXiv.org/abs/cond-mat/0106090. 7.2

[158] Geppert, U., Küker, M., and Page, D., "Temperature distribution in magnetized neutron star crusts", Astron. Astrophys., 426, 267-277, (2004). Related online version (cited on 2 October 2007):

http://arXiv.org/abs/astro-ph/0403441. 71

[159] Giacconi, R., Gursky, H., Paolini, F.R., and Rossi, B.B., "Evidence for X-Rays From Sources Outside the Solar System", Phys. Rev. Lett., 9, 439-443, (1962). 4.1

[160] Ginzburg, V.L., "Superfluidity and Superconductivity in the Universe", J. Stat. Phys., 1, $3-24,(1969)$. 8.1, 8.1

[161] Ginzburg, V.L., and Kirzhnits, D.A., "On superfluidity of neutron stars", Zh. Eksp. Teor. Fiz., 47, 2006, (1964). 8.2, 8.3.4

[162] Ginzburg, V.L., and Kirzhnits, D.A., "On the superfluidity of neutron stars", Sov. Phys. JETP, 20, 1346, (1965). 8.2, 8.3.4

[163] Glampedakis, K., and Andersson, N., "Crust-core coupling in rotating neutron stars", Phys. Rev. D, 74, 044040, (2006). Related online version (cited on 2 October 2007): http://arXiv.org/abs/astro-ph/0411750. 12.5.3

[164] Glampedakis, K., and Andersson, N., "Ekman layer damping of r modes revisited", Mon. Not. R. Astron. Soc., 371, 1311-1321, (2006). Related online version (cited on 2 October 2007): http://arXiv.org/abs/astro-ph/0607105. 12.5.3

[165] Glampedakis, K., Samuelsson, L., and Andersson, N., "Elastic or magnetic? A toy model for global magnetar oscillations with implications for quasi-periodic oscillations during flares", Mon. Not. R. Astron. Soc., 371, L74-L77, (2006). Related online version (cited on 2 October 2007):

http://arXiv.org/abs/astro-ph/0605461. 12.6

[166] Glendenning, N.K., ed., Compact stars: nuclear physics, particle physics, and general relativity, Astronomy and Astrophysics Library, (Springer, New York, U.S.A.; Berlin, Germany, 2000), 2nd edition. 6.2, 12.6

[167] Gnedin, O.Y., Yakovlev, D.G., and Potekhin, A.Y., "Thermal relaxation in young neutron stars", Mon. Not. R. Astron. Soc., 324, 725-736, (2001). Related online version (cited on 2 October 2007):

http://arXiv.org/abs/astro-ph/0012306. 12.2.1, 69, 70

[168] Gögelein, P., and Müther, H., "Nuclear matter in the crust of neutron stars", Phys. Rev. C, 76, 024312, (2007). Related online version (cited on 2 October 2007): http://arXiv.org/abs/0704.1984. 3.2.4 
[169] Gori, G., Ramponi, F., Barranco, F., Broglia, R.A., Colò, G., Sarchi, D., and Vigezzi, E., "Excitation modes and pairing interaction in the inner crust of a neutron stars", Nucl. Phys. A, 731, 401-408, (2004). 8.2.3

[170] Goriely, S., Demetriou, P., Janka, H.-T., Pearson, J.M., and Samyn, M., "The r-process nucleosynthesis: a continued challenge for nuclear physics and astrophysics", Nucl. Phys. A, 758, 587-594, (2005). Related online version (cited on 2 October 2007): http://arXiv.org/abs/astro-ph/0410429. 12.3

[171] Goriely, S., Samyn, M., Pearson, J.M., and Onsi, M., "Further explorations of SkyrmeHartree-Fock-Bogoliubov mass formulas. IV: Neutron-matter constraint", Nucl. Phys. A, 750, 425-443, (2005). 10

[172] Gorkov, L.P., and Melik-Barkhudarov, T.K., "Contribution to the theory of super-fluidity in an imperfect Fermi gas", Sov. Phys. JETP, 13, 1018, (1961). 8.2.1

[173] Gorter, C.J., and Mellink, J.H., "On the irreversible processes in liquid helium II", Physica, 15, 285-304, (1949). 8.3.5

[174] Gourgoulhon, E., "An Introduction to Relativistic Hydrodynamics", in Rieutord, M., and Dubrulle, B., eds., Stellar Fluid Dynamics and Numerical Simulations: From the Sun to Neutron Stars, Aussois and Cargèse, France, September 2004 and May 2005, EAS Publications Series, vol. 21, pp. 43-79, (EDP Sciences, Les Ulis, France, 2006). 10.1

[175] Greenstein, G., "Superfluid turbulence in neutron stars", Nature, 227, 791-794, (1970). 8.3.5

[176] Grigorian, H., "Brightness constraint for cooling models of young neutron stars", Phys. Rev. $C, \mathbf{7 4}, 025801,(2006)$. Related online version (cited on 2 October 2007): http://arXiv.org/abs/astro-ph/0507052. 12.2.2

[177] Grindlay, J., Gursky, H., Schnopper, H., Parsignault, D.R., Heise, J., Brinkman, A.C., and Schrijver, J., "Discovery of intense X-ray bursts from the globular cluster NGC 6624", Astrophys. J., 205, L127-L130, (1976). 4.1

[178] Gupta, S., Brown, E.F., Schatz, H., Möller, P., and Kratz, K.-L., "Heating in the Accreted Neutron Star Ocean: Implications for Superburst Ignition", Astrophys. J., 662, 1188-1197, (2007). Related online version (cited on 2 October 2007): http://arXiv.org/abs/astro-ph/0609828. 4.3, 12.7

[179] Gusakov, M.E., Yakovlev, D.G., Haensel, P., and Gnedin, O.Y., "Direct Urca process in a neutron star mantle", Astron. Astrophys., 421, 1143-1148, (2004). Related online version (cited on 2 October 2007):

http://arXiv.org/abs/astro-ph/0404165. 3.3, 11.8, 11.8.1, 11.8.1

[180] Haberl, F., Turolla, R., de Vries, C.P., Zane, S., Vink, J., Méndez, M., and Verbunt, F., "Evidence for precession of the isolated neutron star RX J0720.4-3125", Astron. Astrophys., 451, L17-L21, (2006). Related online version (cited on 2 October 2007):

http://arXiv.org/abs/astro-ph/0603724. 8.3.5, 12.5.2

[181] Haensel, P., Kaminker, A.D., and Yakovlev, D.G., "Electron neutrino-antineutrino bremsstrahlung in a liquid phase of neutron star crusts", Astron. Astrophys., 314, 328340, (2003). Related online version (cited on 28 April 2008):

http://arXiv.org/abs/astro-ph/9604073. 11.5, 11.5 
[182] Haensel, P., Levenfish, K.P., and Yakovlev, D.G., "Bulk viscosity in superfluid neutron star cores. III. Effects of $\Sigma^{-}$hyperons", Astron. Astrophys., 381, 1080-1089, (2002). Related online version (cited on 28 April 2008):

http://arXiv.org/abs/astro-ph/0110575. 12.5.3

[183] Haensel, P., and Pichon, B., "Experimental nuclear masses and the ground state of cold dense matter", Astron. Astrophys., 283, 313-318, (1994). Related online version (cited on 2 October 2007):

http://arXiv.org/abs/nucl-th/9310003. 3, 3.2, 5.1, 5.1, 5.1, 42, 43, 61

[184] Haensel, P., Potekhin, A.Y., and Yakovlev, D.G., Neutron Stars 1: Equation of State and Structure, Astrophysics and Space Science Library, vol. 326, (Springer, New York, U.S.A., 2007). 1, 2.1, 2, 3.1, 3.1, 3.2.1, 5.1, 29, 30, 33, 5.3, 6.1, 6.2, 6.3, 41, 6.4, 8.2, 56, 64, 12.4 .1

[185] Haensel, P., and Zdunik, J.L., "Equation of state and structure of the crust of an accreting neutron star", Astron. Astrophys., 229, 117-122, (1990). 3, 4.2, 19, 5.2, 39, 43, 55

[186] Haensel, P., and Zdunik, J.L., "Non-equilibrium processes in the crust of an accreting neutron star", Astron. Astrophys., 227, 431-436, (1990). 19, 4.3

[187] Haensel, P., and Zdunik, J.L., "Nuclear composition and heating in accreting neutron-star crusts", Astron. Astrophys., 404, L33-L36, (2003). Related online version (cited on 2 October 2007):

http://arXiv.org/abs/astro-ph/0305220. 4.2, 21, 4.2, 4.3

[188] Haensel, P., and Zdunik, J.L., "Models of crustal heating in accreting neutron stars", Astron. Astrophys., 480, 459-464, (2008). Related online version (cited on 16 June 2008): http://arXiv.org/abs/0708.3996. 4.3, 22, 23, 4.3, 24, 4.3, 85

[189] Harding, D., Guyer, R.A., and Greenstein, G., "Superfluidity in neutron stars. III. Relaxation processes between the superfluid and the crust", Astrophys. J., 222, 991-1005, (1978).

ADS: http://adsabs.harvard.edu/abs/1978ApJ . . 222 . .991H. 8.3.5

[190] Hartle, J.B., "Slowly Rotating Relativistic Stars. I. Equations of Structure", Astrophys. J., 150, 1005-1029, (1967). 6.2, 6.2

[191] Hashimoto, M., Seki, H., and Yamada, M., "Shape of Nuclei in the Crust of Neutron Star", Prog. Theor. Phys., 71, 320-326, (1984).

ADS: http://adsabs.harvard.edu/abs/1984PThPh..71..320H. 3.3

[192] Haskell, B., Jones, D.I., and Andersson, N., "Mountains on neutron stars: accreted versus non-accreted crusts", Mon. Not. R. Astron. Soc., 373, 1423-1439, (2006). Related online version (cited on 2 October 2007):

http://arXiv.org/abs/astro-ph/0609438. 12.5.1

[193] Haskell, B., Samuelsson, L., Glampedakis, K., and Andersson, N., "Modelling magnetically deformed neutron stars", Mon. Not. R. Astron. Soc., 385, 531-542, (2008). Related online version (cited on 16 June 2008):

http://arXiv.org/abs/0705.1780. 12.5.1, 12.5.1

[194] Heinz, S., Schulz, N.S., Brandt, W.N., and Galloway, D.K., "Evidence of a Parsec-Scale X-Ray Jet from the Accreting Neutron Star Circinus X-1", Astrophys. J., 663, L93-L96, (2007). Related online version (cited on 2 October 2007): http://arXiv.org/abs/0706.3881. 12.3 
[195] Hessels, J.W.T., Ransom, S.M., Stairs, I.H., Freire, P.C.C., Kaspi, V.M., and Camilo, F., "A Radio Pulsar Spinning at 716 Hz", Science, 311, 1901-1904, (2006). Related online version (cited on 2 October 2007):

http://arXiv.org/abs/astro-ph/0601337. 12.4

[196] Heyl, J.S., "r-Modes on Rapidly Rotating, Relativistic Stars. I. Do Type I Bursts Excite Modes in the Neutron Star Ocean?", Astrophys. J., 600, 939-945, (2004). Related online version (cited on 2 October 2007):

http://arXiv.org/abs/astro-ph/0108450. 12.7.1

[197] Hillebrandt, W., Wolff, R.G., and Nomoto, K., "Supernova explosions of massive stars. The mass range 8 to $10 M_{\odot} "$, Astron. Astrophys., 133, 175-184, (1984).

ADS: http://adsabs.harvard.edu/abs/1984A\%26A...133..175H. 35

[198] Hirasawa, M., and Shibazaki, N., "Vortex Configurations, Oscillations, and Pinning in Neutron Star Crusts", Astrophys. J., 563, 267-275, (2001). Related online version (cited on 2 October 2007): http://arXiv.org/abs/astro-ph/0109196. 8.3.5

[199] Hobbs, G., Lyne, A.G., Joshi, B.C., Kramer, M., Stairs, I.H., Camilo, F., Manchester, R.N., D'Amico, N., Possenti, A., and Kaspi, V.M., "A very large glitch in PSR J1806-2125", Mon. Not. R. Astron. Soc., 333, L7-L10, (2002). Related online version (cited on 2 October 2007): http://arXiv.org/abs/astro-ph/0204445. 12.4, 75

[200] Horner, H., "Scattering Function S(Q,w) for Solid Helium", Phys. Rev. Lett., 29, 556-558, (1972). 8.3.1

[201] Horowitz, C.J., Pérez-García, M.A., Berry, D.K., and Piekarewicz, J., "Dynamical response of the nuclear 'pasta' in neutron star crusts", Phys. Rev. C, 72, 035801, (2005). Related online version (cited on 2 October 2007):

http://arXiv.org/abs/nucl-th/0508044. 3.3, 12.1

[202] Hund, F., "Materie unter sehr hohen Drucken und Temperaturen", Ergeb. Exakten Naturwiss., 15, 189-228, (1936). 1

[203] Hund, F., "Matter under very high pressures and temperatures", in Riffert, H., Müther, H., Herold, H., and Ruder, H., eds., Matter at High Densities in Astrophysics: Compact Stars and the Equation of State, Springer Tracts in Modern Physics, vol. 133, pp. 217-257, (Springer, Berlin, Germany; New York, U.S.A., 1996). 1

[204] Hüttner, B., "A new method for the determination of the optical mass of electrons in metals", J. Phys.: Cond. Matter, 8, 11041-11052, (1996). 8.3.7

[205] Iida, K., and Sato, K., "Spin-down of Neutron Stars and Compositional Transitions in the Cold Crustal Matter", Astrophys. J., 477, 294, (1997). Related online version (cited on 2 October 2007): http://arXiv.org/abs/astro-ph/9609093. 6.3

[206] Iida, K., Watanabe, G., and Sato, K., "Formation of Nuclear 'Pasta' in Cold Neutron Star Matter", Prog. Theor. Phys., 106, 551-559, (2001). Related online version (cited on 2 October 2007): http://arXiv.org/abs/nucl-th/0108034. 3.3, 3.3

[207] Iida, K., Watanabe, G., and Sato, K., "Formation of Nuclear 'Pasta' in Cold Neutron Star Matter", Prog. Theor. Phys., 110, 847-848, (2003). 3.3, 3.3 
[208] INFN, "The Virgo Project", project homepage. URL (cited on 20 August 2007): http://www.virgo.infn.it/. 12.5

[209] Inman, C.L., and Ruderman, M.A., "Plasma Neutrino Emission from a Hot, Dense Electron Gas", Astrophys. J., 140, 1025, (1964). 11.3

[210] Institut für Theoretische Physik, "Matthias Hempel's homepage", personal homepage. URL (cited on 20 August 2007):

http://th.physik.uni-frankfurt.de/ hempel/. 5.1

[211] Institute of Astronomy and Astrophysics, Université Libre de Bruxelles, "BRUSLIB: the Brussels Nuclear Library for Astrophysics Applications", online resource. URL (cited on 20 August 2007):

http://www .astro.ulb.ac.be/Html/bruslib.html. 3.1, 3.1

[212] Instituto de Astronomía UNAM, "Neutron Star Theory Group at UNAM", project homepage. URL (cited on 20 August 2007):

http://www.astroscu.unam.mx/neutrones/. 12.2

[213] in’t Zand, J.J.M., Cornelisse, R., Kuulkers, E., Heise, J., Kuiper, L., Bazzano, A., Cocchi, M., Muller, J.M., Natalucci, L., Smith, M.J.S., and Ubertini, P., "The first outburst of SAX J1808.4-3658 revisited", Astron. Astrophys., 372, 916-921, (2001). Related online version (cited on 2 October 2007):

http://arXiv.org/abs/astro-ph/0104285. 12.7.1

[214] Israel, G.L., Belloni, T., Stella, L., Rephaeli, Y., Gruber, D.E., Casella, P., Dall'Osso, S., Rea, N., Persic, M., and Rothschild, R.E., "The Discovery of Rapid X-Ray Oscillations in the Tail of the SGR 1806-20 Hyperflare", Astrophys. J., 628, L53-L56, (2005). Related online version (cited on 2 October 2007):

http://arXiv.org/abs/astro-ph/0505255. 12.6

[215] Itoh, N., Adachi, T., Nakagawa, M., Kohyama, Y., and Munakata, H., "Neutrino Energy Loss in Stellar Interiors. III. Pair, Photo-, Plasma, and Bremsstrahlung Processes", Astrophys. J., 339, 354-364, (1989).

ADS: http://adsabs.harvard.edu/abs/1989ApJ . . 339 . 354I. 11.4

[216] Itoh, N., Adachi, T., Nakagawa, M., Kohyama, Y., and Munakata, H., "Erratum: Neutrino Energy Loss in Stellar Interiors. III. Pair, Photo-, Plasma, and Bremsstrahlung Processes", Astrophys. J., 360, 741, (1993).

ADS: http://adsabs.harvard.edu/abs/1990ApJ . . 360 . 741N. 11.4

[217] Itoh, N., Hayashi, H., Nishikawa, A., and Kohyama, Y., "Neutrino Energy Loss in Stellar Interiors. VII. Pair, Photo-, Plasma, Bremsstrahlung, and Recombination Neutrino Processes", Astrophys. J. Suppl. Ser., 102, 411-424, (1996).

ADS: http://adsabs.harvard.edu/abs/1996ApJS . .102 . .411I. 11.4

[218] Jaikumar, P., Meyer, B.S., Otsuki, K., and Ouyed, R., "Nucleosynthesis in neutron-rich ejecta from quark-novae", Astron. Astrophys., 471, 227-236, (2007). 12.3

[219] Janka, H.-T., Langanke, K., Marek, A., Martínez-Pinedo, G., and Müller, B., "Theory of core-collapse supernovae", Phys. Rep., 442, 38-74, (2007). Related online version (cited on 2 October 2007): http://arXiv.org/abs/astro-ph/0612072. 12.1 
[220] Jaranowski, P., Królak, A., and Schutz, B.F., "Data analysis of gravitational-wave signals from spinning neutron stars: The signal and its detection", Phys. Rev. D, 58(6), 063001, (1998). Related online version (cited on 2 October 2007): http://arXiv.org/abs/gr-qc/9804014. 12.5.1

[221] Jog, C.J., and Smith, R.A., "Mixed lattice phases in cold dense matter", Astrophys. J., 253, 839-841, (1982). 3, 3.1

[222] Jones, P.B., "Rotation of the neutron-drip superfluid in pulsars: temperature-dependence of the resistive force", Mon. Not. R. Astron. Soc., 244, 675-679, (1990). ADS: http://adsabs.harvard.edu/abs/1990MNRAS.244. .675J. 8.3.5

[223] Jones, P.B., "Rotation of the neutron-drip superfluid in pulsars: the Kelvin phonon contribution to dissipation", Mon. Not. R. Astron. Soc., 257, 501-506, (1992).

ADS: http://adsabs.harvard.edu/abs/1992MNRAS . 257 . .501J. 8.3.5

[224] Jones, P.B., "Amorphous and Heterogeneous Phase of Neutron Star Matter", Phys. Rev. Lett., 83, 3589-3592, (1999). 3.4

[225] Jones, P.B., "First-principles point-defect calculations for solid neutron star matter", Mon. Not. R. Astron. Soc., 321, 167-175, (2001). 3.4

[226] Jönsson, B., Lindman, B., Holmberg, K., and Kronberg, B., Surfactants and Polymers in Aqueous Solution, (Wiley, Chichester, U.K.; New York, U.S.A., 1998). 16

[227] Kaaret, P., Prieskorn, Z., Zand, J.J.M.i., Brandt, S., Lund, N., Mereghetti, S., Götz, D., Kuulkers, E., and Tomsick, J.A., "Evidence of $1122 \mathrm{~Hz}$ X-Ray Burst Oscillations from the Neutron Star X-Ray Transient XTE J1739-285", Astrophys. J., 657, L97-L100, (2007). Related online version (cited on 2 October 2007):

http://arXiv.org/abs/astro-ph/0611716. 6.3, 12.7.1

[228] Kaminker, A.D., Gnedin, O.Y., Yakovlev, D.G., Amsterdamski, P., and Haensel, P., "Neutrino emissivity from $e^{-} e^{+}$annihilation in a strong magnetic field: hot, nondegenerate plasma", Phys. Rev. D, 46, 4133-4139, (1992). 11.2

[229] Kaminker, A.D., Haensel, P., and Yakovlev, D.G., "Nucleon superfluidity vs. observations of cooling neutron stars", Astron. Astrophys., 373, L17-L20, (2001). Related online version (cited on 2 October 2007):

http://arXiv.org/abs/astro-ph/0105047. 8.2.1

[230] Kaminker, A.D., Levenfish, K.P., Yakovlev, D.G., Amsterdamski, P., and Haensel, P., "Neutrino emissivity from $e^{-}$synchrotron and $e^{-} e^{+}$annihilation processes in a strong magnetic field: general formalism and nonrelativistic limit", Phys. Rev. D, 46, 3256-3264, (1992). 11.2

[231] Kaminker, A.D., Pethick, C.J., Potekhin, A.Y., Thorsson, V., and Yakovlev, D.G., "Neutrino-pair bremsstrahlung by electrons in neutron star crusts", Astron. Astrophys., 343, 1009-1024, (1999). Related online version (cited on 2 October 2007):

http://arXiv.org/abs/astro-ph/9812447. 11.5, 64

[232] Kaspi, V.M., and Gavriil, F.P., "A Second Glitch from the "Anomalous" X-Ray Pulsar 1RXS J170849.0-4000910”, Astrophys. J., 596, L71-L74, (2003). 12.4

[233] Kaspi, V.M., Gavriil, F.P., Woods, P.M., Jensen, J.B., Roberts, M.S.E., and Chakrabarty, D., "A Major Soft Gamma Repeater-like Outburst and Rotation Glitch in the No-longerso-anomalous X-Ray Pulsar 1E 2259+586", Astrophys. J., 588, L93-L96, (2003). Related 
online version (cited on 2 October 2007):

http://arXiv.org/abs/astro-ph/0304205. 12.4

[234] Kaspi, V.M., Lackey, J.R., and Chakrabarty, D., "A Glitch in an Anomalous X-Ray Pulsar", Astrophys. J., 537, L31-L34, (2000). 12.4

[235] Ketterle, W., "Bose-Einstein condensation in dilute atomic gases: atomic physics meets condensed matter physics", Physica B, 280, 11-19, (2000). 8.3.1

[236] Khalatnikov, I.M., An Introduction to the Theory of Superfluidity, (Addison-Wesley, Redwood City, U.S.A., 1989). 8.3.6

[237] Khan, E., Sandulescu, N., and Giai, N.V., "Collective excitations in the inner crust of neutron stars: Supergiant resonances", Phys. Rev. C, 71, 042801, (2005). Related online version (cited on 2 October 2007): http://arXiv.org/abs/nucl-th/0411056. 3.2.4, 8.2.3, 12.2.1

[238] Kinney, J.B., and Mendell, G., "r-modes in accreting neutron stars with magnetoviscous boundary layers", Phys. Rev. D, 67(2), 024032, (2003). Related online version (cited on 2 October 2007): http://arXiv.org/abs/gr-qc/0206001. 12.5.3

[239] Kirszhnits, D.A., "Superfluidity and superconductivity of neutron pulsar stars", Radiophys. Quantum Electron., 13, 1424-1427, (1970). 8.2.2

[240] Kirzhnits, D.A., "Superconductivity in systems with arbitrary interaction sign", J. Exp. Theor. Phys. Lett., 9, 360-364, (1969).

ADS: http://adsabs.harvard.edu/abs/1969ZhPmR...9..360K. 8.1

[241] Kittel, C., Introduction to Solid State Physics, (Wiley, New York, U.S.A., 1996), 7th edition. $3.2 .4,7.1,8.3 .7$

[242] Kohanoff, J., and Hansen, J.-P., "Statistical properties of the dense hydrogen plasma: An ab initio molecular dynamics investigation", Phys. Rev. E, 54, 768-781, (1996). 3.1

[243] Kohn, W., and Sham, L.J., "Self-Consistent Equations Including Exchange and Correlation Effects", Phys. Rev., 140, 1133-1138, (1965).

ADS: http://adsabs.harvard.edu/abs/1965PhRv. .140.1133K. 3.2.2, 3.2.3

[244] Kokkotas, K.D., and Schmidt, B., "Quasi-Normal Modes of Stars and Black Holes", Living Rev. Relativity, 2, lrr-1999-2, (1999). URL (cited on 2 October 2007): http://www. livingreviews . org/lrr-1999-2. 12.5.2

[245] Kolomeitseiv, E.E., and Voskresensky, D.N., "Neutrino emission due to Cooper-pair recombination in neutron stars reexamined", Phys. Rev. C, 77, 065808, (2008). Related online version (cited on 8 December 2008): http://arXiv.org/abs/0802.1404. 11.6, 11.9

[246] Landau, L.D., "On the Theory of Superfluidity of Helium II", J. Phys. (Moscow), 6, 91-92, (1941). 8.3.1, 8.3.6

[247] Landau, L.D., "The Theory of Superfluidity of Helium II", J. Phys. (Moscow), 5, 71-90, (1941). 8.3.1, 8.3.1, 8.3.1, 8.3.6 
[248] Landau, L.D., and Lifshitz, E.M., The Classical Theory of Fields, Course of Theoretical Physics, vol. 2, (Pergamon Press, Oxford, U.K.; New York, U.S.A., 1975), 4th edition. 6, $6.1,6.1$

[249] Landau, L.D., and Lifshitz, E.M., Theory of Elasticity, (Pergamon Press, Oxford, U.K., 1986). $7,7,7,7.2$

[250] Langanke, K., "Neutrino nucleus reactions in core-collapse supernovae", Prog. Part. Nucl. Phys., 57, 324-333, (2006). 12.1

[251] Larson, M.B., and Link, B., "Simulations of glitches in isolated pulsars", Mon. Not. R. Astron. Soc., 333, 613-622, (2002). Related online version (cited on 2 October 2007): http://arXiv.org/abs/astro-ph/0105461. 12.4.2

[252] Lassaut, M., Flocard, H., Bonche, P., Heenen, P.H., and Suraud, E., "Equation of state of hot dense matter", Astron. Astrophys., 183, L3-L6, (1987). 36

[253] Lattimer, J.M., Mackie, F., Ravenhall, D.G., and Schramm, D.N., "The decompression of cold neutron star matter", Astrophys. J., 213, 225-233, (1977). 12.3

[254] Lattimer, J.M., Prakash, M., Pethick, C.J., and Haensel, P., "Direct URCA process in neutron stars", Phys. Rev. Lett., 66, 2701-2704, (1991). 11.8.1

[255] Lattimer, J.M., and Swesty, F.D., "A generalized equation of state for hot, dense matter", Nucl. Phys. A, 535, 331-376, (1991). 35, 11.8.1, 12.1, 65, 66, 67

[256] Lattimer, J.M., van Riper, K.A., Prakash, M., and Prakash, M., "Rapid cooling and the structure of neutron stars", Astrophys. J., 425, 802-813, (1994). 12.2.1

[257] Lee, U., "Axisymmetric oscillations of magnetic neutron stars", Mon. Not. R. Astron. Soc., 374, 1015-1029, (2007). Related online version (cited on 2 October 2007): http://arXiv.org/abs/astro-ph/0610182. 12.6

[258] Legut, D., Friák, M., and Šob, M., "Why Is Polonium Simple Cubic and So Highly Anisotropic?", Phys. Rev. Lett., 99, 016402, (2007). 3.1

[259] Leinson, L.B., "Neutrino Emission from the Bubble Phase of Stellar Nuclear Matter", Astrophys. J., 415, 759, (1993). 11.8, 11.8.1

[260] Leinson, L.B., "Neutrino Pair Emission from the Inner Crust of a Neutron Star", Space Sci. Rev., 74, 481-484, (1995). 11.8, 11.8 .1

[261] Levenfish, K.P., and Haensel, P., "Nucleon superfluidity versus thermal states of isolated and transiently accreting neutron stars", Astrophys. Space Sci., 308, 457-465, (2007). Related online version (cited on 2 October 2007):

http://arXiv.org/abs/astro-ph/0611115. 83

[262] Levin, Y., "On the theory of magnetar QPOs", Mon. Not. R. Astron. Soc., 377, 159-167, (2007). Related online version (cited on 2 October 2007):

http://arXiv.org/abs/astro-ph/0612725. 12.6

[263] Levin, Y., and Ushomirsky, G., "Crust-core coupling and r-mode damping in neutron stars: a toy model", Mon. Not. R. Astron. Soc., 324, 917-922, (2001). Related online version (cited on 2 October 2007):

http://arXiv.org/abs/astro-ph/0006028. 12.5.3 
[264] Lindblom, L., and Owen, B. J., "Effect of hyperon bulk viscosity on neutron star r-modes", Phys. Rev. D, 65, 063006, (2002). Related online version (cited on 28 April 2008):

http://arXiv.org/abs/astro-ph/0110558. 12.5.3

[265] Lindblom, L., Owen, B.J., and Ushomirsky, G., "Effect of a neutron-star crust on the r-mode instability", Phys. Rev. D, 62, 084030, (2000). Related online version (cited on 2 October 2007):

http://arXiv.org/abs/astro-ph/0006242. 12.5.3

[266] Link, B., and Cutler, C., "Vortex unpinning in precessing neutron stars", Mon. Not. R. Astron. Soc., 336, 211-216, (2002). Related online version (cited on 2 October 2007): http://arXiv.org/abs/astro-ph/0108281. 8.3.5

[267] Link, B., and Epstein, R.I., "Thermally Driven Neutron Star Glitches", Astrophys. J., 457, 844, (1996). Related online version (cited on 2 October 2007): http://arXiv.org/abs/astro-ph/9508021. 12.4.2

[268] Link, B., Epstein, R.I., and Baym, G., "Superfluid vortex creep and rotational dynamics of neutron stars", Astrophys. J., 403, 285-302, (1993).

ADS: http://adsabs.harvard.edu/abs/1993ApJ . . 403. .285L. 12.4 .2

[269] Link, B., Epstein, R.I., and Lattimer, J.M., "Pulsar Constraints on Neutron Star Structure and Equation of State", Phys. Rev. Lett., 83, 3362-3365, (1999). Related online version (cited on 2 October 2007):

http://arXiv.org/abs/astro-ph/9909146. 12.4.4, 12.4.4, 12.4.4, 77

[270] L'Observatoire de Paris, "LORENE: Langage Objet pour la RElativité NumériquE", project homepage. URL (cited on 10 September 2007):

http://www.lorene.obspm.fr/. 6.3

[271] Lombardo, U., and Schulze, H.-J., "Superfluidity in Neutron Star Matter", in Blaschke, D., Glendenning, N.K., and Sedrakian, A., eds., Physics of Neutron Star Interiors, International Workshop on Physics of Neutron Star Interiors, ECT* Trento, Italy, June 19 - July 7, 2000, Lecture Notes in Physics, vol. 578, pp. 30-53, (Springer, Berlin, Germany; New York, U.S.A., 2001). 8.2, $45,8.2 .2$

[272] London, F., "The-phenomenon of liquid helium and the Bose-Einstein degeneracy", Nature, 141, 643-644, (1938). 8.3.2

[273] Lorenz, C.P., Dense Matter and the Compressible Liquid Drop Model, Ph.D. Thesis, (University of Illinois at Urbana-Champaign, Urbana-Champaign, U.S.A., 1991). 3.2.1

[274] Lorenz, C.P., Ravenhall, D.G., and Pethick, C.J., "Neutron star crusts", Phys. Rev. Lett., 70, 379-382, (1993). 3.3, 3.3, 38, 11.8

[275] Lyne, A.G., and Graham-Smith, F., Pulsar Astronomy, Cambridge Astrophysics Series, vol. 31, (Cambridge University Press, Cambridge, U.K.; New York, U.S.A., 1998), 2nd edition. 12.4

[276] Lyne, A.G., Shemar, S.L., and Smith, F.G., "Statistical studies of pulsar glitches", Mon. Not. R. Astron. Soc., 315, 534-542, (2000). 12.4.4

[277] Mackie, F.D., and Baym, G., "Compressible liquid drop nuclear model and mass formula", Nucl. Phys. A, 285, 332-348, (1977). 3.2.1, 6.1, 39 
[278] Magierski, P., and Heenen, P.-H., "Structure of the inner crust of neutron stars: Crystal lattice or disordered phase?", Phys. Rev. C, 65, 045804, (2002). Related online version (cited on 2 October 2007):

http://arXiv.org/abs/nucl-th/0112018. 3.2.2, 3.2.4

[279] Markwardt, C.B., Miller, J.M., and Wijnands, R., "Sax J1808.4-3658", IAU Circ., 2002(7993), (2002). Related online version (cited on 23 June 2008): http://www.cfa.harvard.edu/iauc/07900/07993.html. 12.7.1

[280] Martínez-Pinedo, G., Liebendörfer, M., and Frekers, D., "Nuclear input for core-collapse models", Nucl. Phys. A, 777, 395-423, (2006). Related online version (cited on 2 October 2007):

http://arXiv.org/abs/astro-ph/0412091. 12.1

[281] Maruyama, T., Tatsumi, T., Endo, T., and Chiba, S., "Pasta structures in compact stars", (2006). URL (cited on 2 October 2007): http://arXiv.org/abs/nucl-th/0605075. 3.3

[282] Maruyama, T., Tatsumi, T., Voskresensky, D.N., Tanigawa, T., and Chiba, S., "Nuclear 'pasta' structures and the charge screening effect", Phys. Rev. C, 72, 015802, (2005). Related online version (cited on 2 October 2007):

http://arXiv.org/abs/nucl-th/0503027. 3.1, 3.3

[283] Matsuo, M., "Spatial structure of neutron Cooper pair in low density uniform matter", Phys. Rev. C, 73, 044309, (2006). Related online version (cited on 2 October 2007): http://arXiv.org/abs/nucl-th/0512021. 8.2.3

[284] Matsuzaki, M., "Ordered bicontinuous double-diamond morphology in subsaturation nuclear matter", Phys. Rev. C, 73, 028801, (2006). Related online version (cited on 2 October 2007): http://arXiv.org/abs/nucl-th/0510044. 3.3

[285] McDermott, P.N., van Horn, M., and Hansen, C.J., "Nonradial oscillations of neutron stars", Astrophys. J., 325, 725-748, (1988). 7.1, 12.5.2

[286] McKenna, J., and Lyne, A.G., "PSR1737-30 and period discontinuities in young pulsars", Nature, 343, 349-350, (1990). 12.4.4

[287] Medin, Z., and Lai, D., "Condensed surfaces of magnetic neutron stars, thermal surface emission, and particle acceleration above pulsar polar caps", Mon. Not. R. Astron. Soc., 382, 1833-1852, (2007). Related online version (cited on 16 June 2008): http://arXiv.org/abs/0708.3863. 2.2, 1

[288] Melatos, A., and Peralta, C., "Superfluid Turbulence and Pulsar Glitch Statistics", Astrophys. J., 662, L99-L102, (2007). 8.3.5

[289] Mendell, G., "Magnetic effects on the viscous boundary layer damping of the r-modes in neutron stars", Phys. Rev. D, 64, 044009, (2001). Related online version (cited on 2 October 2007):

http://arXiv.org/abs/gr-qc/0102042. 12.5.3

[290] Messios, N., Papadopoulos, D.B., and Stergioulas, N., "Torsional oscillations of magnetized relativistic stars", Mon. Not. R. Astron. Soc., 328, 1161-1168, (2001). Related online version (cited on 2 October 2007):

http://arXiv.org/abs/astro-ph/0105175. 12.6 
[291] Migdal, A.B., "Superfluidity and the moments of inertia of nuclei", Nucl. Phys., 13, 655, (1959). 8.2

[292] Miralda-Escudé, J., Paczyński, B., and Haensel, P., "Thermal structure of accreting neutron stars and strange stars", Astrophys. J., 362, 572-583, (1990). 25, 26

[293] Mochizuki, Y., Izuyama, T., and Tanihata, I., "Dynamics of Exotic Nuclear Rod Formation for the Origin of Neutron Star Glitches", Astrophys. J., 521, 281-296, (1999). 3.3, 8.2.2

[294] Monrozeau, C., Margueron, J., and Sandulescu, N., "Nuclear superfluidity and cooling time of neutron star crusts", Phys. Rev. C, 75(6), 065807, (2007). Related online version (cited on 2 October 2007):

http://arXiv.org/abs/nucl-th/0703064. 3.2.4, 8.2.2, 12.2.1

[295] Montani, F., May, C., and Müther, H., "Mean field and pairing properties in the crust of neutron stars", Phys. Rev. C, 69, 065801, (2004). Related online version (cited on 2 October 2007):

http://arXiv.org/abs/nucl-th/0401024. 8.2.3

[296] Morii, M., Kawai, N., and Shibazaki, N., "A Pulse Profile Change Possibly Associated with a Glitch in the Anomalous X-Ray Pulsar 4U 0142+61", Astrophys. J., 622, 544-548, (2005). Related online version (cited on 2 October 2007):

http://arXiv.org/abs/astro-ph/0412106. 12.4

[297] Morsch, O., and Oberthaler, M., "Dynamics of Bose-Einstein condensates in optical lattices", Rev. Mod. Phys., 78, 179-215, (2006). 8.3.1

[298] Motokowa, M., "Physics in high magnetic fields", Rep. Prog. Phys., 67, 1995-2052, (2004). 13

[299] MPI for Gravitational Physics (Albert Einstein Institute), "GEO600: The German-British Gravitational Wave Detector", project homepage. URL (cited on 20 August 2007):

http://geo600.aei.mpg.de/. 12.5

[300] Nandkumar, R., and Pethick, C.J., "Transport coefficients of dense matter in the liquid metal regime", Mon. Not. R. Astron. Soc., 209, 511-524, (1984). 9.4

[301] National Astronomical Observatory, "TAMA300: The 300m Laser Interferometer Gravitational Wave Antenna", project homepage. URL (cited on 20 August 2007):

http://tamago.mtk.nao.ac.jp/. 12.5

[302] Negele, J.W., and Vautherin, D., "Density-Matrix Expansion for an Effective Nuclear Hamiltonian", Phys. Rev. C, 5, 1472-1493, (1972). 3.2.3, 3.2.4

[303] Negele, J.W., and Vautherin, D., "Neutron star matter at sub-nuclear densities", Nucl. Phys. A, 207, 298-320, (1973). 3, 9, 3.2.3, 10, 11, 3.2.3, 3, 3.2.3, 4, 12, 3.2.4, 43, 8.2.2, 47, 48, 54, 57,61

[304] Newton, W.G., Stone, J.R., and Mezzacappa, A., "From microscales to macroscales in 3D: selfconsistent equation of state for supernova and neutron star models", J. Phys.: Conf. Ser., 46, 408-412, (2006). Related online version (cited on 2 October 2007):

http://arXiv.org/abs/0708.3197. 3.2.4

[305] Nobel Foundation, "The Nobel Prize in Physics 1974", institutional homepage, (1974). URL (cited on 6 September 2007): http://nobelprize.org/nobel_prizes/physics/laureates/1974/. 12.4 
[306] Nobel Foundation, "The Nobel Prize in Physics 1993", institutional homepage, (1993). URL (cited on 6 September 2007): http://nobelprize.org/nobel_prizes/physics/laureates/1993/. 12.5

[307] Nozières, P., "Is the Roton in Superfluid ${ }^{4}$ He the Ghost of a Bragg Spot?", J. Low Temp. Phys., 137, 45-67, (2004). 8.3.1

[308] Ogasawara, R., and Sato, K., "Nuclei in Neutrino-Degenerate Dense Matter. I - Cold Case -", Prog. Theor. Phys., 68, 222-235, (1982).

ADS: http://adsabs.harvard.edu/abs/1982PThPh. .68. .2220. 10, 3.3

[309] Ogata, S., and Ichimaru, S., "First-principles calculations of shear moduli for Monte Carlosimulated Coulomb solids", Phys. Rev. A, 42, 4867-4870, (1990). 7.1, 7.1, 7.1

[310] Onsager, L., "Statistical hydrodynamics", Nuovo Cimento, 6, 249, (1949). 8.3 .2

[311] Onsi, M., Dutta, A.K., Chatri, H., Goriely, S., Chamel, N., and Pearson, J.M., "Semiclassical equation of state and specific-heat expressions with proton shell corrections for the inner crust of a neutron star", Phys. Rev. C, 77, 065805, (2008). Related online version (cited on 8 December 2008):

http://arXiv.org/abs/0806.0296. 3.2.2, 3.2.2, 11, 3.2.3, 3.2.4

[312] Onsi, M., Przysiezniak, H., and Pearson, J.M., "Equation of state of stellar nuclear matter in the temperature-dependent extended Thomas-Fermi formalism", Phys. Rev. C, 55, 31393148, (1997). 3.2 .2

[313] Oppenheimer, J.R., and Volkoff, G.M., "On Massive Neutron Cores", Phys. Rev., 55, 374381, (1939). 6.1

[314] Oyamatsu, K., "Nuclear shapes in the inner crust of a neutron star", Nucl. Phys. A, 561, 431-452, (1993). 10

[315] Oyamatsu, K., Hashimoto, M., and Yamada, M., "Further Study of the Nuclear Shape in High-Density Matter", Prog. Theor. Phys., 72, 373-375, (1984). 3.3, 17

[316] Oyamatsu, K., and Yamada, M., "Shell energies of non-spherical nuclei in the inner crust of a neutron star", Nucl. Phys. A, 578, 181-203, (1994). 3.2.2

[317] Packard, R.E., "Pulsar speedups related to metastability of the superfluid neutron-star core.", Phys. Rev. Lett., 28, 1080-1082, (1972). 12.4

[318] Page, D., Geppert, U., and Weber, F., "The cooling of compact stars", Nucl. Phys. A, 777, 497-530, (2006). Related online version (cited on 2 October 2007):

http://arXiv.org/abs/astro-ph/0508056. 12.2

[319] Pandharipande, V.R., Pines, D., and Smith, R.A., "Neutron star structure: theory, observation, and speculation.", Astrophys. J., 208, 550-566, (1976). 7.1

[320] Pandharipande, V.R., and Ravenhall, D.G., "Hot Nuclear Matter", in Soyeur, M., Flocard, H., Tamain, B., and Porneuf, M., eds., Nuclear Matter and Heavy Ion Collisions, Proceedings of a NATO Advanced Research Workshop, held February 7 -16, 1989, in Les Houches, France, NATO ASI Series B, vol. 205, p. 103, (Plenum Press, New York, U.S.A., 1989). 5.1

[321] Papenbrock, T., and Bertsch, G.F., "Pairing in low-density Fermi gases", Phys. Rev. C, 59, 2052-2055, (1999). Related online version (cited on 2 October 2007): http://arXiv.org/abs/nucl-th/9811077. 8.2.1 
[322] Penrose, O., and Onsager, L., "Bose-Einstein Condensation and Liquid Helium", Phys. Rev., 104, 576-584, (1956). 8.3.2

[323] Peralta, C., Melatos, A., Giacobello, M., and Ooi, A., "Global Three-dimensional Flow of a Neutron Superfluid in a Spherical Shell in a Neutron Star", Astrophys. J., 635, 1224-1232, (2005). Related online version (cited on 2 October 2007):

http://arXiv.org/abs/astro-ph/0509416. 8.3.5

[324] Peralta, C., Melatos, A., Giacobello, M., and Ooi, A., "Transitions between Turbulent and Laminar Superfluid Vorticity States in the Outer Core of a Neutron Star", Astrophys. J., 651, 1079-1091, (2006). Related online version (cited on 2 October 2007): http: //arXiv.org/abs/astro-ph/0607161. 8.3.5, 12.4.3

[325] Pethick, C.J., and Potekhin, A.Y., "Liquid crystals in the mantles of neutron stars", Phys. Lett. B, 427, 7-12, (1998). Related online version (cited on 2 October 2007): http://arXiv.org/abs/astro-ph/9803154. 3.3, 7.2, 7.2, 7.2

[326] Pethick, C.J., and Ravenhall, D.G., "Matter at large neutron excess and the physics of neutron-star crusts", Annu. Rev. Nucl. Part. Sci., 45, 429-484, (1995). 3.1, 9, 3.3, 3.3, 8.1

[327] Pethick, C.J., Ravenhall, D.G., and Lattimer, J.M., "Effect of nuclear curvature energy on the transition between nuclei and bubbles in dense matter", Phys. Lett. B, 128, 137-140, (1983). 3.3

[328] Piro, A.L., "Shear Waves and Giant-Flare Oscillations from Soft Gamma-Ray Repeaters", Astrophys. J. Lett., 634, L153-L156, (2005). Related online version (cited on 2 October 2007): http://arXiv.org/abs/astro-ph/0510578. 12.6

[329] Piro, A.L., and Bildsten, L., "Neutron Star Crustal Interface Waves", Astrophys. J., 619, 1054-1063, (2005). Related online version (cited on 2 October 2007): http://arXiv.org/abs/astro-ph/0410197. 12.7.1

[330] Piro, A.L., and Bildsten, L., "Surface Modes on Bursting Neutron Stars and X-Ray Burst Oscillations", Astrophys. J., 629, 438-450, (2005). Related online version (cited on 2 October 2007):

http://arXiv.org/abs/astro-ph/0502546. 12.7.1

[331] Piro, A.L., and Bildsten, L., "The Energy Dependence of Neutron Star Surface Modes and X-Ray Burst Oscillations", Astrophys. J., 638, 968-937, (2006). Related online version (cited on 2 October 2007):

http://arXiv.org/abs/astro-ph/0510663. 12.7.1

[332] Pizzochero, P.M., Barranco, F., Vigezzi, E., and Broglia, R.A., "Nuclear Impurities in the Superfluid Crust of Neutron Stars: Quantum Calculation and Observable Effects on the Cooling", Astrophys. J., 569, 381-394, (2002). 12.2.1

[333] Pizzochero, P.M., Viverit, L., and Broglia, R.A., "Vortex-Nucleus Interaction and Pinning Forces in Neutron Stars", Phys. Rev. Lett., 79, 3347-3350, (1997). Related online version (cited on 2 October 2007): http://arXiv.org/abs/astro-ph/9709060. 8.3.5

[334] Pomeau, Y., and Rica, S., "Dynamics of a model of supersolid", Phys. Rev. Lett., 72, 24262429, (1994). 8.3.1 
[335] Pontecorvo, B.M., Zh. Eksp. Teor. Fiz., 36, 1615, (1959). 11.5

[336] Pontecorvo, B.M., Sov. Phys. JETP, 9, 1148, (1959). 11.5

[337] Postnov, K.A., and Yungelson, L.R., "The Evolution of Compact Binary Star Systems", Living Rev. Relativity, 9, lrr-2006-6, (2006). URL (cited on 2 October 2007): http://www.livingreviews.org/lrr-2006-6. 12.3

[338] Potekhin, A.Y., "Electron conduction in magnetized neutron star envelopes", Astron. Astrophys., 351, 787-797, (1999). Related online version (cited on 12 June 2008): http://arXiv.org/abs/astro-ph/9909100. 59, 9.5, 9.5.2, 9.5.3

[339] Potekhin, A.Y., Baiko, D.A., Haensel, P., and Yakovlev, D.G., "Transport properties of degenerate electrons in neutron star envelopes and white dwarf cores", Astron. Astrophys., 346, 345-353, (1999). Related online version (cited on 26 May 2008): http://arXiv.org/abs/astro-ph/9903127. 53, 9.2, 9.3

[340] Prakash, M., Lattimer, J.M., and Ainsworth, T.L., "Equation of state and the maximum mass of neutron stars", Phys. Rev. Lett., 61, 2518-2521, (1988). 83

[341] Prigogine, I., Introduction to thermodynamics of irreversible processes, (Interscience Publishers, New York, U.S.A., 1960). 4.3

[342] Prix, R., "Variational description of multifluid hydrodynamics: Uncharged fluids", Phys. Rev. D, 69, 043001, (2004). Related online version (cited on 2 October 2007): http://arXiv.org/abs/physics/0209024. 10.1

[343] Prix, R., "Variational description of multifluid hydrodynamics: Coupling to gauge fields", Phys. Rev. D, 71, 083006, (2005). Related online version (cited on 2 October 2007): http://arXiv.org/abs/physics/0503217. 10.1

[344] Ravenhall, D.G., Bennett, C.D., and Pethick, C.J., "Nuclear surface energy and neutron-star matter.", Phys. Rev. Lett., 28, 978-981, (1972). 9

[345] Ravenhall, D.G., Pethick, C.J., and Wilson, J.R., "Structure of Matter below Nuclear Saturation Density", Phys. Rev. Lett., 50, 2066-2069, (1983). 3.3

[346] Ring, P., and Schuck, P., The Nuclear Many-Body Problem, (Springer, Berlin, Germany; New York, U.S.A., 1980). 8.2.1

[347] Ritus, V.I., "Photoproduction of neutrinos of electrons and the neutrino radiation from stars", Zh. Eksp. Teor. Fiz., 41, 1285-1293, (1961). In Russian. 11.4

[348] Ritus, V.I., "Photoproduction of neutrinos of electrons and the neutrino radiation from stars", Sov. Phys. JETP, 14, 915, (1961). 11.4

[349] Ruderman, M., "Neutron star quakes and pulsar periods", Nature, 223, 597, (1969). 12.4.1

[350] Ruderman, M., "Pulsars: Structure and Dynamics", Annu. Rev. Astron. Astrophys., 10, 427, (1972). 12.4

[351] Ruderman, M., "Crust-breaking by neutron superfluids and the VELA pulsar glitches", Astrophys. J., 203, 213-222, (1976). 12.4.2, 12.4 .3

[352] Ruderman, M., "Neutron star crustal plate tectonics. I. Magnetic dipole evolution in millisecond pulsars and low-mass X-ray binaries", Astrophys. J., 366, 261-269, (1991). ADS: http://adsabs.harvard.edu/abs/1991ApJ . . 366. .261R. 12.4.2, 12.4.3 
[353] Ruderman, M., "Neutron star crustal plate tectonics. III. Cracking, glitches, and gamma-ray bursts", Astrophys. J., 382, 587-593, (1991).

ADS: http://adsabs.harvard.edu/abs/1991ApJ . . 382 . 587R. 12.4.2, 12.4.3

[354] Ruderman, M., Zhu, T., and Chen, K., "Neutron Star Magnetic Field Evolution, Crust Movement, and Glitches", Astrophys. J., 492, 267, (1998). 8.3.4, 12.4 .2

[355] Ruderman, M., Zhu, T., and Chen, K., "Neutron Star Magnetic Field Evolution, Crust Movement, and Glitches: Erratum", Astrophys. J., 502, 1027, (1998). 8.3.4, 12.4.2

[356] Ruderman, R., "Neutron star crustal plate tectonics. II. Evolution of radio pulsar magnetic fields", Astrophys. J., 382, 576-586, (1991).

ADS: http://adsabs.harvard.edu/abs/1991ApJ . . 382 . 576R. 12.4.2, 12.4.3

[357] Rüster, S.B., Hempel, M., and Schaffner-Bielich, J., "Outer crust of nonaccreting cold neutron stars", Phys. Rev. C, 73, 035804, (2006). 2, 3.2, 5.1, 27, 28, 5.1, 31

[358] Rutledge, R.E., Bildsten, L., Brown, E.F., Pavlov, G.G., Zavlin, V.E., and Ushomirsky, G., "Crustal Emission and the Quiescent Spectrum of the Neutron Star in KS 1731-260", Astrophys. J., 580, 413-422, (2002). Related online version (cited on 2 October 2007): http://arXiv.org/abs/astro-ph/0108125. 12.7.2, 12.7.3

[359] Samuelsson, L., and Andersson, N., "Neutron star asteroseismology. Axial crust oscillations in the Cowling approximation", Mon. Not. R. Astron. Soc., 374, 256-268, (2007). Related online version (cited on 2 October 2007):

http://arXiv.org/abs/astro-ph/0609265. 12.6, 12.6, 79, 80

[360] Sandulescu, N., "Nuclear superfluidity and specific heat in the inner crust of neutron stars", Phys. Rev. C, 70, 025801, (2004). Related online version (cited on 2 October 2007): http://arXiv.org/abs/nucl-th/0403019. 3.2.4, 8.2.3

[361] Sandulescu, N., van Giai, N., and Liotta, R.J., "Superfluid properties of the inner crust of neutron stars", Phys. Rev. C, 69, 045802, (2004). Related online version (cited on 2 October 2007):

http://arXiv.org/abs/nucl-th/0402032. 8.2.3, 12.2.1

[362] Sato, K., "Nuclear Compositions in the Inner Crust of Neutron Stars", Prog. Theor. Phys., 62, 957-968, (1979). 4.3

[363] Sauls, J.A., "Superfluidity in the Interiors of Neutrons Stars", in Ögelman, H., and van den Heuvel, E.P.J., eds., Timing Neutron Stars, Proceedings of the NATO Advanced Study Institute on Timing Neutron Stars, Çesme, Izmir, Turkey, 4-15 April 1988, NATO ASI Series C, vol. 262, pp. 441-490, (Kluwer Academic Press, Dordrecht, Netherlands; Boston, U.S.A., 1989). 8, 8.2, 8.3.7

[364] Schatz, H., Aprahamian, A., Barnard, V., Bildsten, L., Cumming, A., Ouellette, M., Rauscher, T., Thielemann, F.-K., and Wiescher, M., "End Point of the rp Process on Accreting Neutron Stars", Phys. Rev. Lett., 86, 3471-3474, (2001). Related online version (cited on 2 October 2007):

http://arXiv.org/abs/astro-ph/0102418. 3.4, 4.2

[365] Schatz, H., Bildsten, L., Cumming, A., and Wiescher, M., "The Rapid Proton Process Ashes from Stable Nuclear Burning on an Accreting Neutron Star", Astrophys. J., 524, 1014-1029, (1999). Related online version (cited on 2 October 2007): http://arXiv.org/abs/astro-ph/9905274. 3.4 
[366] Schwenk, A., Friman, B., and Brown, G.E., "Renormalization group approach to neutron matter: quasiparticle interactions, superfluid gaps and the equation of state", Nucl. Phys. A, 713, 191-216, (2003). Related online version (cited on 2 October 2007): http://arXiv.org/abs/nucl-th/0207004. 5, 8.2.2, 47, 8.2.3, 48

[367] Sedrakian, A., and Clark, J.W., "Nuclear Superconductivity in Compact Stars: BCS Theory and Beyond", in Sedrakian, A., Clark, J.W., and Alford, M., eds., Pairing in Fermionic Systems: Basic Concepts and Modern Applications, Series on Advances in Quantum ManyBody Theory, vol. 8, pp. 135-174, (World Scientific, Singapore; Hackensack, U.S.A., 2006). Related online version (cited on 2 October 2007): http://arXiv.org/abs/nucl-th/0607028. 8, 8.2, 12.4 .3

[368] Sedrakian, A., Muether, H., and Schuck, P., "Vertex renormalization of weak interactions and Cooper-pair breaking in cooling compact stars", Phys. Rev. C, 76, 055805, (2007). 11.6, 11.9

[369] Sedrakian, A.D., and Sedrakian, D.M., "Superfluid Core Rotation in Pulsars. I. Vortex Cluster Dynamics", Astrophys. J., 447, 305, (1995). 12.4.2

[370] Sedrakian, A.D., Sedrakian, D.M., Cordes, J.M., and Terzian, Y., "Superfluid Core Rotation in Pulsars. II. Postjump Relaxations", Astrophys. J., 447, 324, (1995). 12.4 .2

[371] Shabanova, T.V., Lyne, A.G., and Urama, J.O., "Evidence for Free Precession in the Pulsar B1642-03", Astrophys. J., 552, 321-325, (2001). Related online version (cited on 2 October 2007): http: //arXiv.org/abs/astro-ph/0101282. 8.3.5, 12.5.2

[372] Shaham, J., "Free precession of neutron stars: Role of possible vortex pinning", Astrophys. J., 214, 251-260, (1977).

ADS: http://adsabs.harvard.edu/abs/1977ApJ . . 214. .251S. 8.3.5

[373] Shapiro, S.L., and Teukolsky, S.A., Black Holes, White Dwarfs, and Neutron Stars: The Physics of Compact Objects, (Wiley, New York, U.S.A., 1983). 3.1, 4.2, 5.4, 12.1, 12.5.1, 12.5 .2

[374] Shen, H., Toki, H., Oyamatsu, K., and Sumiyoshi, K., "Relativistic equation of state of nuclear matter for supernova and neutron star", Nucl. Phys. A, 637, 435-450, (1998). Related online version (cited on 2 October 2007):

http://arXiv.org/abs/nucl-th/9805035. 35, 12.1, 65, 66, 67

[375] Shen, H., Toki, H., Oyamatsu, K., and Sumiyoshi, K., "Relativistic Equation of State of Nuclear Matter for Supernova Explosion", Prog. Theor. Phys., 100, 1013-1031, (1998). Related online version (cited on 2 October 2007):

http://arXiv.org/abs/nucl-th/9806095. 35, 12.1, 65, 66, 67

[376] Shimizu, K., Kimura, T., Furomoto, S., Takeda, K., Kontani, K., Onuki, Y., and Amaya, K., "Superconductivity in the non-magnetic state of iron under pressure", Nature, 412, 316-318, (2001). 8.1

[377] Shternin, P.S., "Shear viscosity of degenerate electron matter", J. Phys. A, 41, 205501, (2008). Related online version (cited on 12 June 2008):

http://arXiv.org/abs/0803.3893. 9.4 
[378] Shternin, P.S., and Yakovlev, D.G., "Electron thermal conductivity owing to collisions between degenerate electrons", Phys. Rev. D, 74, 043004, (2006). Related online version (cited on 12 June 2008):

http://arXiv.org/abs/astro-ph/0608371. 9.2, 9.3

[379] Shternin, P.S., Yakovlev, D.G., Haensel, P., and Potekhin, A.Y., "Neutron star cooling after deep crustal heating in the X-ray transient KS 1731-260", Mon. Not. R. Astron. Soc., 382, L43-L47, (2007). Related online version (cited on 16 June 2008): http://arXiv.org/abs/0708.0086. 12.7.3, 85, 86

[380] Slater, J.C., "A Simplification of the Hartree-Fock Method", Phys. Rev., 81, 385-390, (1951). 3.2 .3

[381] Šlaus, I., Akaishi, Y., and Tanaka, H., "Neutron-neutron effective range parameters", Phys. Rep., 173, 257-300, (1989). 8.2.1

[382] Smoluchowski, R., "Frequency of pulsar starquakes", Phys. Rev. Lett., 24, 923-925, (1970). 12.5.1

[383] Sonin, E.B., "Vortex oscillations and hydrodynamics of rotating superfluids", Rev. Mod. Phys., 59, 87-155, (1987). 8.3.5

[384] Sonoda, H., Watanabe, G., Sato, K., Takiwaki, T., Yasuoka, K., and Ebisuzaki, T., "Impact of nuclear 'pasta' on neutrino transport in collapsing stellar cores", Phys. Rev. C, 75, 042801, (2007). Related online version (cited on 2 October 2007): http://arXiv.org/abs/astro-ph/0701364. 3.3, 12.1

[385] Sotani, H., Kokkotas, K.D., and Stergioulas, N., "Torsional oscillations of relativistic stars with dipole magnetic fields", Mon. Not. R. Astron. Soc., 375, 261-277, (2007). Related online version (cited on 2 October 2007):

http://arXiv.org/abs/astro-ph/0608626. 12.6

[386] Spitkovsky, A., Levin, Y., and Ushomirsky, G., "Propagation of Thermonuclear Flames on Rapidly Rotating Neutron Stars: Extreme Weather during Type I X-Ray Bursts", Astrophys. J., 566, 1018-1038, (2002). Related online version (cited on 2 October 2007): http://arXiv.org/abs/astro-ph/0108074. 12.7.1, 82

[387] Stairs, I.H., Lyne, A.G., and Shemar, S.L., "Evidence for free precession in a pulsar", Nature, 406, 484-486, (2000). 8.3.5, 12.5.2

[388] Stergioulas, N., "Rapidly Rotating Neutron Star (RNS code)", project homepage, University of Wisconsin-Milwaukee Center for Gravitation and Cosmology. URL (cited on 10 September 2007):

http://www.gravity.phys. uwm.edu/rns/. 6.3

[389] Stergioulas, N., "Rotating Stars in Relativity", Living Rev. Relativity, 6, lrr-2003-3, (2003). URL (cited on 2 October 2007):

http://www. livingreviews .org/lrr-2003-3. 6.3

[390] Sterne, T.E., "The equilibrium theory of the abundance of the elements: a statistical investigation of assemblies in equilibrium in which transmutations occur", Mon. Not. R. Astron. Soc., 93, 736-766, (1933). 1

[391] Stone, J.R., and Reinhard, P.-G., "The Skyrme interaction in finite nuclei and nuclear matter", Prog. Part. Nucl. Phys., 58, 587-657, (2007). 3.2.4 
[392] Strohmayer, T., and Bildsten, L., "New views of thermonuclear bursts", in Lewin, W., and van der Klis, M., eds., Compact Stellar X-Ray Sources, Cambridge Astrophysics Series, vol. 39, pp. 113-156, (Cambridge University Press, Cambridge, U.K., 2006). Related online version (cited on 2 October 2007):

http://arXiv.org/abs/astro-ph/0301544. 12.7.1

[393] Strohmayer, T., van Horn, H.M., Ogata, S., Iyetomi, H., and Ichimaru, S., "The shear modulus of the neutron star crust and nonradial oscillations of neutron stars", Astrophys. J., 375, 679-686, (1991). 7.1

[394] Strohmayer, T.E., and Markwardt, C.B., "Evidence for a Millisecond Pulsar in 4U 1636-53 during a Superburst", Astrophys. J., 577, 337-345, (2002). Related online version (cited on 2 October 2007):

http://arXiv.org/abs/astro-ph/0205435. 12.7.1

[395] Strohmayer, T.E., Markwardt, C.B., Swank, J.H., and in't Zand, J., "X-Ray Bursts from the Accreting Millisecond Pulsar XTE J1814-338", Astrophys. J., 596, L67-L70, (2003). Related online version (cited on 2 October 2007):

http: //arXiv.org/abs/astro-ph/0308353. 12.7.1

[396] Strohmayer, T.E., and Watts, A.L., "Discovery of Fast X-Ray Oscillations during the 1998 Giant Flare from SGR 1900+14", Astrophys. J., 632, L111-L114, (2005). Related online version (cited on 2 October 2007):

http://arXiv.org/abs/astro-ph/0508206. 12.6

[397] Strohmayer, T.E., and Watts, A.L., "The 2004 Hyperflare from SGR 1806-20: Further Evidence for Global Torsional Vibrations", Astrophys. J., 653, 593-601, (2006). Related online version (cited on 2 October 2007):

http://arXiv.org/abs/astro-ph/0608463. 12.6

[398] Strohmayer, T.E., Zhang, W., and Swank, J.H., "363 Hz Oscillations during the Rising Phase of Bursts from 4U 1728-34: Evidence for Rotational Modulation", Astrophys. J. Lett., 487, L77-L80, (1997). 12.7.1

[399] Strohmayer, T.E., Zhang, W., Swank, J.H., Smale, A., Titarchuk, L., Day, C., and Lee, U., "Millisecond X-Ray Variability from an Accreting Neutron Star System", Astrophys. J. Lett., 469, L9, (1996). 81

[400] Sumiyoshi, K., Oyamatsu, K., and Toki, H., "Neutron star profiles in the relativistic Brueckner-Hartree-Fock theory", Nucl. Phys. A, 595, 327-345, (1995).

ADS: http://adsabs.harvard.edu/abs/1995NuPhA.595..327S. 10

[401] Sumiyoshi, K., Yamada, S., Suzuki, H., and Hillebrandt, W., "The fate of a neutron star just below the minimum mass: does it explode?", Astron. Astrophys., 334, 159-168, (1998). Related online version (cited on 2 October 2007):

http://arXiv.org/abs/astro-ph/9707230. 12.3

[402] Sumiyoshi, K., Yamada, S., Suzuki, H., Shen, H., Chiba, S., and Toki, H., "Postbounce Evolution of Core-Collapse Supernovae: Long-Term Effects of the Equation of State", Astrophys. J., 629, 922-932, (2005). Related online version (cited on 2 October 2007): http://arXiv.org/abs/astro-ph/0506620. 12.1, 65, 66, 67

[403] Tilley, D.R., and Tilley, J., Superfluidity and Superconductivity, (Wiley, New York, U.S.A., 1974). 9.3 
[404] Tisza, L., "Transport Phenomena in Helium II", Nature, 141, 913, (1938). 8.3.6

[405] Tkachenko, V.K., "On vortex lattices", Zh. Eksp. Teor. Fiz., 49, 1875, (1965). In Russian. 8.3.2

[406] Tkachenko, V.K., "On vortex lattices", Sov. Phys. JETP, 22, 1282-1286, (1966). 8.3.2

[407] Tolman, R.C., "Static Solutions of Einstein's Field Equations for Spheres of Fluid", Phys. Rev., 55, 364-373, (1939). 6.1

[408] Tsakadze, D.S., and Tsakadze, S.D., "Simulation of pulsar behavior in a low-temperature laboratory (Review)", Astrofizika, 15, 533-547, (1979). In Russian. 12.4

[409] Tsakadze, J.S., and Tsakadze, S.J., "Properties of slowly rotating helium II and the superfluidity of pulsars", J. Low Temp. Phys., 39, 649-688, (1980). 12.4

[410] Vartanyan, Y.L., and Ovakimova, N.K., "Cold evaporation of neutrons from nuclei in superdense matter", Biurakanskaia Obs. Soobshcheniia, 49, 87-95, (1976). In Russian. 4.3

[411] Vavoulidis, M., Stavridis, A., Kokkotas, K.D., and Beyer, H., "Torsional oscillations of slowly rotating relativistic stars", Mon. Not. R. Astron. Soc., 377, 1553-1556, (2007). Related online version (cited on 2 October 2007):

http://arXiv.org/abs/gr-qc/0703039. 12.6

[412] Ventura, J., and Potekhin, A., "Neutron Star Envelopes and Thermal Radiation from the Magnetic Surface", in Kouveliotou, C., Ventura, J., and van den Heuvel, E., eds., The Neutron Star-Black Hole Connection, Proceedings of the NATO Advanced Study Institute, Elounda, Crete, Greece, 7-18 June 1999, NATO Science Series C, vol. 567, pp. 393-414, (Kluwer Academic Publishers, Dordrecht, Netherlands; Boston, U.S.A., 2001). 9.5

[413] Vigezzi, E., Barranco, F., Broglia, R.A., Colò, G., Gori, G., and Ramponi, F., "Pairing correlations in the inner crust of neutron stars", Nucl. Phys. A, 752, 600-603, (2005). 3.2.4, 8.2 .3

[414] Villain, L., and Haensel, P., "Non-equilibrium beta processes in superfluid neutron star cores", Astron. Astrophys., 444, 539-548, (2005). Related online version (cited on 24 April 2008):

http://arxiv.org/abs/astro-ph/0504572. 10.2

[415] Vinen, W.F., "The Detection of Single Quanta of Circulation in Liquid Helium II", Proc. R. Soc. London, Ser. A, 260, 218-236, (1961). 8.3.2

[416] Wang, N., Manchester, R.N., Pace, R.T., Bailes, M., Kaspi, V.M., Stappers, B.W., and Lyne, A.G., "Glitches in southern pulsars", Mon. Not. R. Astron. Soc., 317, 843-860, (2000). 12.4

[417] Watanabe, G., "Understanding nuclear 'pasta': current status and future prospects", (2006). URL (cited on 2 October 2007):

http://arXiv.org/abs/nucl-th/0610116. 3.3

[418] Watanabe, G., and Iida, K., "Electron screening in the liquid-gas mixed phases of nuclear matter", Phys. Rev. C, 68, 045801, (2003). Related online version (cited on 2 October 2007): http://arXiv.org/abs/nucl-th/0308071. 3.1

[419] Watanabe, G., Iida, K., and Sato, K., "Thermodynamic properties of nuclear 'pasta' in neutron star crusts", Nucl. Phys. A, 676, 455-473, (2000). Related online version (cited on 2 October 2007):

http://arXiv.org/abs/astro-ph/0001273. 3.3, 3.3, 7.2 
[420] Watanabe, G., Iida, K., and Sato, K., "Erratum to: I. 'Thermodynamic properties of nuclear 'pasta' in neutron star crusts' [Nucl. Phys. A 676 (2000) 455]", Nucl. Phys. A, 726, 357-365, (2003). 3.3, 3.3, 7.2

[421] Watts, A.L., and Strohmayer, T.E., "Detection with RHESSI of High-Frequency X-Ray Oscillations in the Tail of the 2004 Hyperflare from SGR 1806-20", Astrophys. J., 637, L117-L120, (2006). Related online version (cited on 2 October 2007):

http://arXiv.org/abs/astro-ph/0512630. 12.6

[422] Wigner, E., and Seitz, F., "On the Constitution of Metallic Sodium", Phys. Rev., 43, 804$810,(1933)$. 3.2.3, 3.2.4

[423] Williams, G.A., and Packard, R.E., "Photographs of Quantized Vortex Lines in Rotating He II", Phys. Rev. Lett., 33, 280-283, (1974). 8.3.2

[424] Wolf, R.A., "Some Effects of the Strong Interactions on the Properties of Neutron-Star Matter", Astrophys. J., 145, 834, (1966). 8.2

[425] Wong, T., Backer, D.C., and Lyne, A.G., "Observations of a Series of Six Recent Glitches in the Crab Pulsar", Astrophys. J., 548, 447-459, (2001). Related online version (cited on 2 October 2007):

http://arXiv.org/abs/astro-ph/0010010. 74

[426] Woods, P.M., and Thompson, C., "Soft gamma repeaters and anomalous X-ray pulsars: magnetar candidates", in Lewin, W., and van der Klis, M., eds., Compact Stellar X-Ray Sources, Cambridge Astrophysics Series, vol. 39, pp. 547-586, (Cambridge University Press, Cambridge, U.K., 2006). Related online version (cited on 2 October 2007): http://arXiv.org/abs/astro-ph/0406133. 12.6

[427] Yakovlev, D.G., Gasques, L., and Wiescher, M., "Pycnonuclear burning of ${ }^{34}$ Ne in accreting neutron stars", Mon. Not. R. Astron. Soc., 371, 1322-1326, (2006). 4.2

[428] Yakovlev, D.G., Kaminker, A.D., Gnedin, O.Y., and Haensel, P., "Neutrino emission from neutron stars", Phys. Rep., 354, 1-155, (2001). Related online version (cited on 2 October 2007):

http://arXiv.org/abs/astro-ph/0012122. 8.2.2, 10.2, 11.1, 11.2, 11.2, 11.3, 11.5, 11.6, $11.7,11.8,61,62,63,64,68,12.2 .1$

[429] Yakovlev, D.G., Levenfish, K.P., and Haensel, P., "Thermal state of transiently accreting neutron stars", Astron. Astrophys., 407, 265-271, (2003). Related online version (cited on 2 October 2007):

http://arXiv.org/abs/astro-ph/0209027. 12.7.2

[430] Yakovlev, D.G., Levenfish, K.P., Potekhin, A.Y., Gnedin, O.Y., and Chabrier, G., "Thermal states of coldest and hottest neutron stars in soft X-ray transients", Astron. Astrophys., 417, 169-179, (2004). Related online version (cited on 2 October 2007):

http://arXiv.org/abs/astro-ph/0310259. 12.7.2

[431] Yakovlev, D.G., and Pethick, C.J., "Neutron Star Cooling", Annu. Rev. Astron. Astrophys., 42, 169-210, (2004). Related online version (cited on 2 October 2007):

http://arXiv.org/abs/astro-ph/0402143. 12.2

[432] Yarmchuk, E.J., Gordon, M.J.V., and Packard, R.E., "Observation of Stationary Vortex Arrays in Rotating Superfluid Helium", Phys. Rev. Lett., 43, 214-217, (1979). 8.3.2 
[433] Yu, Y., and Bulgac, A., "Spatial Structure of a Vortex in Low Density Neutron Matter", Phys. Rev. Lett., 90, 161101, (2003). Related online version (cited on 2 October 2007): http://arXiv.org/abs/nucl-th/0212072. 8.3.5

[434] Zeilinger, A., Shull, C.G., Horne, M.A., and Finkelstein, K.D., "Effective mass of neutrons diffracting in crystals", Phys. Rev. Lett., 57, 3089-3092, (1986). 8.3.7

[435] Ziman, J.M., Electrons and Phonons, Oxford Classic Texts in the Physical Sciences, (Clarendon Press / Oxford University Press, Oxford, U.K.; New York, U.S.A., 2001). 9.1, 9.2, 9.2, 9.2 